\title{
Monograph of Cercosporoid fungi from Thailand
}

\section{Phengsintham $\mathbf{P}^{1,2^{*}}$, Braun $\mathbf{U}^{3}$, McKenzie EHC ${ }^{4}$, Chukeatirote $\mathrm{E}^{1}$, Cai $\mathrm{L}^{5}$ and Hyde $\mathrm{KD}^{1}$}

\author{
${ }^{1}$ School of Science, Mae Fah Luang University, Chiang Rai 57100. Thailand \\ ${ }^{2}$ Biology Department, Faculty of Sciences, National University of Laos \\ ${ }^{3}$ Martin-Luther-Universität, Institut für Biologie, Bereich Geobotanik und Botanischer Garten, Herbarium, Neuwerk 21 \\ 06099 Halle/S. Germany \\ ${ }^{4}$ Landcare Research, Private Bag 92170, Auckland, New Zealand \\ ${ }^{5}$ State Key Laboratory of Mycology, Institute of Microbiology, Chinese Academy of Sciences, Beijing 100101, P.R. \\ China.
}

Phengsintham P, Braun U, McKenzie EHC, Chukeatirote E, Cai L, Hyde KD 2013 - Monograph of Cercosporoid fungi from Thailand. Plant Pathology \& Quarantine 3(2), 67-138, doi $10.5943 / \mathrm{ppq} / 3 / 2 / 2$

The diversity of cercosporoid fungi in northern Thailand is very high. Eighty-five cercosporoid species were found in northern Thailand including (i) 84 species of true cercosporoid fungi: Cercospora (34), Passalora (7), Pseudocercospora (42), Zasmidium (1); (ii) One morphological similar fungus. Three new species were established, namely Pseudocercospora christellae, $P$. cratevae and $P$. radermachericola, while 23 cercosporoid species represent new records for Thailand. In this study, 50 species are described in full descriptions and illustrations, and another 35 species are only listed additionally because they have been described in "Monograph of Cercosporid from Laos" or have previously been recorded from Thailand. The data show that the diversity of cercosporoid fungi in northern Thailand is very high; Meeboon (2009) recorded 166 cercosporoid species from this region.

Key words - Asia - Cercospora - Cercospora-like hyphomycetes - taxonomic treatment

\section{Article Information}

Received 10 February 2013

Accepted 6 April 2013

Published online 10 September 2013

*Corresponding author: P. Phengsintham -e-mail-p.phengsintham@ gmail.com

\section{Introduction}

\section{Background of Thai biodiversity}

Thailand is situated in a hot and humid climatic zone which supports a variety of tropical ecosystems. Tropical ecosystems provide more niches for organisms than in temperate ecosystems and hence, support a much larger variety of plant, animal and microbe species. Thailand has approximately 15,000 vascular plants, including 1000 orchid varieties, 600 ferns and over 1,000 endermic species (Tanticharoen, 2004). A database of
Thai fungi compiled by BIOTEC lists 13,696 fungal collections, consisting of 2,200 species in approximately 800 genera (Hywel-Jones \& Boonpratuang, 2001).

\section{Background on cercosporoid fungi from Thailand}

The first significant list of fungi from Thailand was published by Rostrup (1902) and listed 94 taxa including 2 myxomycetes, 58 basidiomycetes, 12 ascomycetes and anamorphic fungi, 14 of which were new species. 
There have been several recent comprehensive accounts of the fungi of Thailand which are among the best documented in the region (Shirouzu et al., 2009; To-anun et al., 2009; Wannathes et al., 2009). Some studies include pathogenic microfungi (Prihastuti et al., 2009; Than et al., 2008). Cercosporoid fungi of Thailand have been well examined since 1980. Sontirat et al. (1980) recorded 21 species of Cercospora. Giatgong (1980) listed 60 cercosporoids, including 13 unidentified species of Cercospora in the host index of plant diseases in Thailand, and Petcharat \& Kajanamaneesathian (1989) recorded 49 cercosporoid species. Nakashima et al. (2007) described three new species and recorded 11 species that were new to Thailand. Forty-three cercosporoid species were included in an annotated list of cercosporoid fungi in northern Thailand (Meeboon et al., 2007), and two taxa associated with necrotic leaflets of areca palms (Areca catechu) were published by To-anun et al. (2009). A Ph.D. thesis by Meeboon (2009) encompassed 166 cercosporoids from northern Thailand. To-anun et al. (2010) reported Cercospora cristellae, a new cercosporoid species associated with the weed Cristella parasitica from northern Thailand. To-anun et al. (2011) included 24 representatives of Cercospora apii s. lat. Three species, Cercospora arecacearum (To-anun et al., 2009), C. neobougainvilleae (Meeboon et al., 2008) and C. habenariicola (Meeboon et al., 2007), have been validly published as new species from Thailand.

\section{Materials and Methods}

\section{Sample collections}

Leaves of plants with leaf spots or other lesions were collected during the course of field trips. Photos of symptoms, including the fungal colonies or fruit bodies were taken. The specimens were collected in three provinces in northern Thailand [Chiang Mai, Chiang Rai and Pha Yao Provinces] (Fig. 1).

\section{Examination of fungal structures}

Macroscopic characters were observed using a stereoscope to check (1) lesions/leaf spots (shape, size, colour, margin), and (2) colonies/caespituli (with details, e.g., amphigenous/epiphyllous, punctiform/pustulate/inconspicuous, effuse, loose, dense, brown/blackish, etc.).

Microscopic examination, measurement, description, and presentation of drawings follow the standard procedures outlined by Braun (1995). In the illustrations, thin-walled structures are depicted by a single line, thickwalled ones by double lines, and stippling is used to accentuate shape and pigmentation.

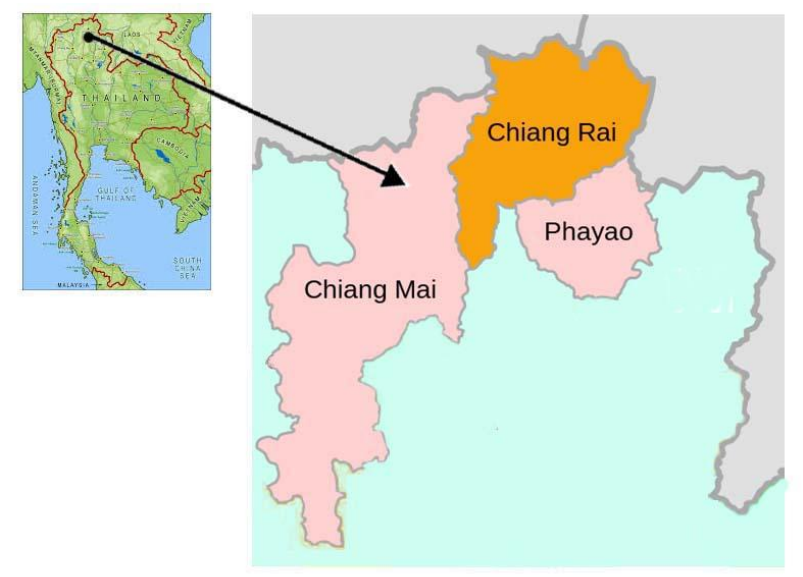

Fig. 1 - Collection sites

\section{Measurements and microscopic study}

Where sufficient material was available, 30 measurements of each morphological character were carried out and the average estimated by using the formula:

$$
\left(\bar{x}=\frac{\sum M}{n} \mu \mathrm{m}\right),
$$

Notes: $\mathrm{m}=$ is a size of each components, $\mathrm{n}=$ is a number of components.

The characters described and/or measured are mycelia (internal, external), hyphae (branched or not, width, septation, colour, wall thin/thick, smooth/verruculose), stromata (location, e.g., substomatal, intraepidermal; shape, size, colour; cells, angular or rounded in outline, size, wall thick/thin), conidiophores (formation, solitary/fasciculate/ sporodochial, arising from internal/external hyphae/stromata, erumpent/through stomata; shape; size; septation; colour; wall, thin/thick, smooth/verrucuose), conidiogenous cells (integrated, terminal/intercalary; length, shape, 
e.g., cylindrical/geniculate/sinuous), conidiogenous loci [scars] (shape, size, thickened, darkened/pigmented or unthickend or inconspicuous, etc.), and conidia (formation, solitary/catenate; shape; size; septation; colour; wall, thin/thick, smooth/verruculose, apex; base; hila, size, thickened/unthickened, pigmented or not).

\section{Identification of Fungi}

The concept of Crous \& Braun (2003) about the classification of Cercospora and morphologically similar cercosporoid genera is followed. The species of cercosporoid hyphomycetes from Thailand were determined on the basis of the currently relevant taxonomic publications, especially the monograph of Cercospora by Chupp (1954), and the works of Deighton (1967-1983), Ellis (1971, 1976), Hsieh \& Goh (1990), Guo \& Hsieh (1995), etc.

\section{Single spore isolation}

Symptomatic leaves were collected from different locations and different species in Thailand. Spores of hyphomycetes of some species were picked directly from the substrate using fine forceps or a needle. The spores of some species were placed in sterilized water and agitated in order to provide a spore suspension (Choi et al., 1999).

The suspension was prepared on sterilized glass slides. Sixteen squares were marked on the bottom of a water agar plate and the prepared spore suspension was then transferred with a sterilised pipette onto the surface of the water agar plate, above each of the drawn squares. Alternatively about six drops of the suspension can be pipetted onto the centre of the agar plate and this can be carefully shaken to spread the suspension. If this method is followed it is a good practice to mark the outer edge of the suspension on the base of the Petri dish. Both of these methods may help to locate the germinating spores later. A small drop of the suspension should be used at this stage to make a permanent slide and to check that the correct fungus has been selected. The unsealed plate is incubated at $25^{\circ} \mathrm{C}$ for $12-24$ hours. The spores were checked within 12 hours and then every 24 hours to establish germination. Once the spores had germinated, a sterilised glass needle was used to pick up a small piece of agar containing a spore. In order to establish that the spore is the one desired, and maintain quality control, a slide is prepared and examined under the compound microscope. If the spores do not germinate after 12 hours, seal the plate with Parafilm and examine periodically. Ten germinated spores are transferred and distributed evenly onto two PDA plates and incubated at $25^{\circ} \mathrm{C}$ until their colony diam. are about 1 to $2 \mathrm{~cm}$. A small piece of mycelium with agar can then be cut and transferred to another PDA plate and the culture is checked after few days, if there is no contamination, a pure culture has been obtained. Cultures can then be stored on the desired media.

Isolates of single spores were deposited in the culture collection at Herbarium, Biology Department, School of Science, Mae Fah Luang University (Thailand), BIOTECH (Thailand), and CBS (The Netherlands).

\section{Herbarium specimens}

Dried specimens were prepared and stored in the herbaria of the Mae Fah Luang University, Chiang Rai, Thailand and the Biology Department, Faculty of Science, National University of Laos. Various duplicates are preserved in the herbarium of the Institute of Biology, Geobotany and Botanical Garden, Halle (Saale), Germany (HAL).

\section{Results}

\section{Summary of results}

By integrating the morphological and molecular characters, 85 cercosporoid species were found in this study including (i) 84 species of true cercosporoid fungi: Cercospora (34), Passalora (7), Pseudocercospora (42), Zasmidium (1); (ii) about 1 species of morphological similar fungi. Three new taxa were established, namely Pseudocercospora christellae, $P$. cratevae and $P$. radermachericola. 
Table 1 - Cercosporoid species found in this study

\begin{tabular}{|c|c|c|c|c|}
\hline Ser\# & Fungus & Hosts & Host Family & Thailand \\
\hline 1 & Cercospora arecacearum & \multirow{2}{*}{$\begin{array}{l}\text { Areca } \mathrm{sp} . \\
\text { Impatiens balsamina, } \\
\text { walleriana }\end{array}$} & Arecaceae & \\
\hline 2 & Cercospora balsaminiana & & Balsaminaceae & \\
\hline 3 & Cercospora basellae-albae & Basella rubra & Basellaceae & \\
\hline 4 & Cercospora broussonetiae & Broussonetia papyrifera & Moraceae & New record \\
\hline 5 & Cercospora buteae & \multirow{2}{*}{$\begin{array}{l}\text { Butea monosperma } \\
\text { Lablab purpureus subsp. } \\
\text { bengalensis }\end{array}$} & Fabaceae & New record \\
\hline 6 & Cercospora canescens & & Fabaceae & \\
\hline 7 & Cercospora codiaei & Cordiaeum variegatum & Euphorbiaceae & \\
\hline 8 & Cercospora coffeicola & \multirow{2}{*}{$\begin{array}{l}\text { Coffea arabica } \\
\text { Crotalaria uncinella subsp. } \\
\text { elliptica }\end{array}$} & Rubiaceae & \\
\hline 9 & Cercospora crotalariae & & Fabaceae & \\
\hline 10 & Cercospora diplaziicola & Diplazium esculentum & Woodsiaceae & New record \\
\hline 11 & Cercospora gossypina & Gossypium herbaceum & Malvaceae & \\
\hline 12 & Cercospora malloti & Mallotus repandus & Euphorbiaceae & New record \\
\hline 13 & Cercospora nilghirensis & Conyza bonariensis & Asteraceae & \\
\hline 14 & Cercospora passifloricola & Passiflora foetida & Passifloraceae & \\
\hline 15 & $\begin{array}{l}\text { Cercospora senecionis- } \\
\text { walkeri }\end{array}$ & Senecio walkeri & Asteraceae & New record \\
\hline 16 & Cercospora sidicola & Sida mysorensis & Malvaceae & New record \\
\hline 17 & Cercospora sonchi & Taraxacum officinale & Asteraceae & \\
\hline 18 & Cercospora sp. sp.1 & Crateva religiosa & Capparaceae & New record \\
\hline 19 & Cercospora sp.sp. 2 & Celtis timoriensis & Cannabaceae & New record \\
\hline 20 & Cercospora sp. sp. 3 & Ziziphus sp & Rhamnaceae & New record \\
\hline 21 & Cercospora tageteae & Tagetes patula & Asteraceae & \\
\hline 22 & Cercospora verniciferae & Spondias pinnata & Anacardiaceae & New record \\
\hline 23 & Passalora barretoana & $\begin{array}{l}\text { Echinochloa esculenta and } \\
\text { Panicum sp. }\end{array}$ & Poaceae & New record \\
\hline 24 & Passalora broussonetiae & Broussonetia papyrifera & Moraceae & New record \\
\hline 25 & $\begin{array}{l}\text { Passalora fusimaculans } \\
\text { Pseudocercospora }\end{array}$ & Agrostis sp. & Poaceae & New record \\
\hline 26 & $\begin{array}{l}\text { atromarginalis } \\
\text { Pseudocercospora }\end{array}$ & Lycianthes biflora & Solanaceae & New record \\
\hline 27 & balsaminae & Impatiens balsamina & Balsaminaceae & \\
\hline 28 & $\begin{array}{l}\text { Pseudocercospora bischofiae } \\
\text { Pseudocercospora }\end{array}$ & Bischofia javanica & Euphorbiaceae & \\
\hline 29 & carbonacea & Dioscorea bulbifera & Dioscoreaceae & \\
\hline 30 & $\begin{array}{l}\text { Pseudocercospora christellae } \\
\text { Pseudocercospora }\end{array}$ & Christella parasitica & Thelypteridaceae & New species \\
\hline 31 & consociata & Justicia gendarussa & Acanthaceae & \\
\hline 32 & Pseudocercospora cratevae & Crateva religiosa & Capparaceae & New speces \\
\hline 33 & Pseudocercospora cycleae & Cyclea peltata & Menispermaceae & \\
\hline 34 & Pseudocercospora jahnii & Tabebuia chrysotricha & Bignoniaceae & \\
\hline 35 & $\begin{array}{l}\text { Pseudocercospora lygodii } \\
\text { Pseudocercospora }\end{array}$ & Lygodium flesuoxum & Schizaeaceae & \\
\hline 36 & malloticola & Mallotus barbatus & Euphorbiaceae & \\
\hline 37 & Pseudocercospora mombin & Spondias pinnata & Anacardiaceae & \\
\hline 38 & Pseudocercospora mori & Molus alba & Moraceae & \\
\hline
\end{tabular}




\begin{tabular}{|c|c|c|c|c|}
\hline Ser\# & Fungus & Hosts & Host Family & Thailand \\
\hline 39 & Psudocercospora olacicola & Olax scandens & Olacaceae & New record \\
\hline 40 & Pseudocercospora oroxyli & Oroxylum indicum & Bignoniaceae & \\
\hline 41 & Pseudocercospora paederiae & Paederia tomentosa & Rubiaceae & \\
\hline 42 & Pseudocercospora panacis & Polyscias balfouriana & Araliaceae & \\
\hline 43 & Pseudocercospora puderi & Rosa chinensis & Rosaceae & \\
\hline 44 & Pseudocercospora punicae & Punica granatum & Lythraceae & \\
\hline 45 & $\begin{array}{l}\text { Pseudocercospora } \\
\text { radermachericola }\end{array}$ & Radermachera ignea & Bignoniaceae & New species \\
\hline 46 & $\begin{array}{l}\text { Pseudocercospora riachueli } \\
\text { var. horiana }\end{array}$ & Vitis venifera & Vitaceae & New record \\
\hline 47 & $\begin{array}{l}\text { Pseudocercospora } \\
\text { scopariicola }\end{array}$ & Scoparia dulcis & Plantaginaceae & New record \\
\hline 48 & Pseudocercospora timorensis & Operculina sp. & Convolvulaceae & New record \\
\hline 49 & Pseudocercospora tremicola & Trema orientalis & Cannabaceae & \\
\hline 50 & $\begin{array}{l}\text { Zasmidium cassiicola } \\
\text { Additional List }\end{array}$ & Cassia fistula & Fabaceae & New record \\
\hline 51 & Cercospora alocasiae & Alocasia macrorrhiza & Araceae & \\
\hline 52 & Cercospora begoniae & Begonia inflata & Begoniaceae & \\
\hline 53 & Cercospora bidentis & Bidens pilosa & Asteraceae & \\
\hline 54 & Cercospora brassicicola & Brassica integrifolia & Brassicaceae & \\
\hline 55 & Cercospora capsicigena & Capsicum anпиum & Solanaceae & \\
\hline 56 & Cercospora cocciniae & Coccinia grandis & Cucurbitaceae & \\
\hline 57 & Cercospora duranticola & Duranta repens & Verbenaceae & New record \\
\hline 58 & Cercospora erechtitis & Erechtites valerianifolius & Asteraceae & \\
\hline 59 & Cercospora ipomoeae & $\begin{array}{l}\text { Ipomoea involucrata, } \\
\text { I. aquatica }\end{array}$ & Convolvulaceae & \\
\hline 60 & Cercospora physalidis & Physalis angulata & Solanaceae & \\
\hline 61 & Cercospora sp. & Oroxylum indicum & Bignoniaceae & \\
\hline 62 & Cercospora zinniae & Zinnia elegans & Asteraceae & \\
\hline 63 & Passalora aenea & Senna siamea & Fabaceae & \\
\hline 64 & Passalora bougainvilleae & Bougainvillea spectabilis & Nyctaginaceae & \\
\hline 65 & Passalora henningsii & Manihot esculenta & Euphorbiaceae & \\
\hline 66 & Passalora perfoliati & Chromolaena sp. & Asteraceae & \\
\hline 67 & Pseudocercospora catappae & Terminalia alata & Combretaceae & \\
\hline 68 & $\begin{array}{l}\text { Pseudocercospora } \\
\text { centrosematicola }\end{array}$ & Centrosema sp. & Fabaceae & \\
\hline 69 & Pseudocercospora cotizensis & $\begin{array}{l}\text { Crotalaria uncinella subsp. } \\
\text { elliptica }\end{array}$ & Fabaceae & \\
\hline 70 & $\begin{array}{l}\text { Pseudocercospora } \\
\text { duabangae }\end{array}$ & Duabanga grandiflora & Lythraceae & New record \\
\hline 71 & $\begin{array}{l}\text { Pseudocercospora eupatorii- } \\
\text { formosani }\end{array}$ & Chromolaena odorata & Asteraceae & \\
\hline 72 & Pseudocercospora fuligena & Lycopersicon esculentum & Solanaceae & \\
\hline 73 & Pseudocercospora getoniae & Getonia floribunda & Combretaceae & New record \\
\hline 74 & Pseudocercospora jussiaeae & Ludwigia prostrata & Onagraceae & \\
\hline 75 & $\begin{array}{l}\text { Pseudocercospora } \\
\text { lythracearum }\end{array}$ & Lagerstroemia macrocarpa & Lythraceae & \\
\hline 76 & Pseudocercospora musae & Musa paradisiaca & Musaceae & \\
\hline 77 & Pseudocercospora & Eucalyptus sp. & Myrtaceae & \\
\hline
\end{tabular}


Plant Pathology \& Quarantine - Doi 10.5943/ppq/3/2/2

\begin{tabular}{|c|c|c|c|c|}
\hline Ser\# & Fungus & Hosts & Host Family & Thailand \\
\hline & paraguayensis & & & \\
\hline 78 & $\begin{array}{l}\text { Pseudocercospora } \\
\text { puerariicola }\end{array}$ & Pueraria phaseoloides & Fabaceae & \\
\hline 79 & $\begin{array}{l}\text { Pseudocercospora } \\
\text { sphaerellae-eugeniae }\end{array}$ & Syzygium cuminii & Myrtaceae & \\
\hline 80 & Pseudocercospora stahlii & Passiflora foetida & Passifloraceae & \\
\hline 81 & $\begin{array}{l}\text { Pseudocercospora stizolobii } \\
\text { Pseudocercospora }\end{array}$ & Mucuna pruriens & Fabaceae & \\
\hline 82 & trichophila var. punctata & Solanum undatum & Solanaceae & \\
\hline 83 & Pseudocercospora wrightiae & Wrightia pubescens & Apocynaceae & \\
\hline 84 & $\begin{array}{l}\text { Zasmidium suregadae } \\
\text { Morphological similar fungi }\end{array}$ & Suregada multiflora & Euphorbiaceae & New record \\
\hline 85 & Cladosporium colocasiae & Colocasia esculenta & Araceae & \\
\hline
\end{tabular}

23 cercosporoid species represent new records for Thailand.

In this study, 50 species are described with full descriptions and illustrations, and another 35 species are only listed because they have previously been recorded from Thailand (Table 1).

\section{Key}

Crous \& Braun (2003) prepared two different keys, viz., a key to the recognised true cercosporoid genera, i.e. dematiaceous hyphomycetious anamorphs of Mycosphaerella with holoblastic conidiogenesis, and a second key to cercosporoid and morphological similar, noncercosporoid genera.

\section{Key to recognized true cercosporoid genera}

This key only contains the true cercosporoid genera discussed and treated in this work (adapted from Crous \& Braun, 2003). The synopsis to identify species is arranged by host families in alphabetical order, based on models applied by Chupp (1954), Ellis (1971, 1976), Deighton (1967-1983), Hsieh \& Goh (1990), and Guo \& Hsieh (1995).

1. Conidiogenous loci inconspicuous or subdenticulate, but always unthickened and not darkened or subconspicuous, i.e. unthickened, but somewhat refractive or rarely very slightly darkened or only outer rim slightly darkened or refractive (visible as minute rings).

Pseudocercospora (III)

1. Conidiogenous loci conspicuous, i.e. thickened and darkened throughout, only with a minute central pore.........................2
2. With verruculose superficial mycelium; conidia amero- to scolecosporous, mostly verruculose.....................Zasmidium (IV)

2. If superficial mycelium present, hyphae smooth or almost so............................ 3

3. Conidia hyaline or subhyaline, usually scolecosporous, acicular, obclavate-cylindrical, filiform, and pluriseptate, rarely nonscolecosporous.....................Cercospora (I) 3. Conidia pigmented, non-scolecosporous, ellipsoid-ovoid, short cylindrical, fusoid and only few septa, to obclavate-cylindrical and pluriseptate. Passalora (II)

\section{Genus Cercospora \\ Anacardiaceae}

Single species; on Spondias

\section{Arecaceae}

Single species; on Areca

Cercospora arecacearum (1)

\section{Asteraceae $=$ Compositae}

On Conyza: Stromata $10-40 \mu \mathrm{m}$ in diam.; conidiophores 50-128 × 3-5 $\mu \mathrm{m}$, unbranched, geniculate; conidia 14-128 $\times 2-3.5 \mu \mathrm{m}, 1-15-$ septate.............Cercospora nilghirensis (13)

On Senecio: Stromata 10-25 $\mu \mathrm{m}$ in diam.; conidiophores $67-170 \times 5-6 \mu \mathrm{m}$, unbranched, geniculate; conidia 17-82 × 4-7 $\mu \mathrm{m}, 0-8-$ septate......Cercospora senecionis-walkeri (15) On Tagetes: Stromata 10-20 $\mu \mathrm{m}$ in diam.; conidiophores $25-68 \times 5-6 \mu \mathrm{m}$, unbranched, geniculate; conidia 45-54 × 5-6 $\mu \mathrm{m}, 1-3-$ septate.................Cercospora tageteae (21) On Taraxacum: Stromata $18-40 \mu \mathrm{m}$ in diam.; conidiophores $15-190 \times 4-5 \mu \mathrm{m}$, unbranched, geniculate; conidia 78-91 × 2-4 $\mu \mathrm{m}, 7-14-$ 
septate. Cercospora sonchi (17)

\section{Balsaminaceae}

Single species; on Impatiens. . . ....................Cercospora balsaminiana (2)

\section{Basellaceae}

Single species; on Basella. Cercospora basellae-albae (3)

\section{Cannabaceae}

Single species; on Celtis. Cercospora sp. sp.2 (19)

\section{Capparaceae}

Single species; on Crateva

\section{Euphorbiaceae}

Cercospora sp. sp. 1 (18)

On Codiaeum: Stromata 17-27 $\mu \mathrm{m}$ in diam.; conidiophores 16-49 × 3-5 $\mu \mathrm{m}$, unbranched, geniculate; conidia 50-150 × 3-5 $\mu \mathrm{m}, 3-8$ septate...................Cercospora codiaei (7) On Mallotus: Stromata $15-30 \mu \mathrm{m}$ in diam.; conidiophores 32-140 × 5-6 $\mu \mathrm{m}$, unbranched, geniculate; conidia 20-146 × 2-4 $\mu \mathrm{m}, 6-19$ septate. Cercospora malloti (12)

\section{Fabaceae}

On Butea: Stromata 18-25 $\mu \mathrm{m}$ in diam.; conidiophores 56-235 × 5-6 $\mu \mathrm{m}$, unbranched, geniculate; conidia $60-110 \times 2-4 \mu \mathrm{m}, 4-5-$ septate Cercospora buteae (5) On Clitoria: Stromata $10-38 \mu \mathrm{m}$ in diam.; conidiophores $28-78 \times 4-6 \mu \mathrm{m}$, unbranched, not geniculate; conidia 42-165 × 3-4 $\mu \mathrm{m}, 3-$ 14-septate ............Cercospora canescens (6) On Crotalaria: Stromata 25-45 $\mu \mathrm{m}$ in diam.; conidiophores 20-148 × 5-6 $\mu \mathrm{m}$, unbranched, geniculate; conidia 40-320 × 3-4 $\mu \mathrm{m}, 3-26-$ septate...............Cercospora crotalariae (9)

On Labla; see Cercospora canescens

On Vigna; see Cercospora canescens

\section{Malvaceae}

On Gossypium: Stromata 13-30 $\mu \mathrm{m}$ in diam.; conidiophores 50-95 × 3.5-6 $\mu \mathrm{m}$, unbranched, geniculate; conidia $32-75 \times 2-4 \mu \mathrm{m}, 1-7$ septate................Cercospora gossypina (11) On Sida: Stromata 7-25 $\mu \mathrm{m}$ in diam.; conidiophores 50-131 × 4-5 $\mu \mathrm{m}$, unbranched, geniculate; conidia 44-105 × 2-6 $\mu \mathrm{m}, 4-8$ septate..................Cercospora sidicola (16)

\section{Moraceae}

Single species; on Broussonetia.

Cercospora broussonetiae (4)

\section{Rhamnaceae}

Single species; on Ziziphus.

\section{Rubiaceae}

Single species; on Coffea

\section{Woodsiaceae (Fern)}

Cercospora coffeicola $(8)$

Single species; on Diplazium

.Cercospora diplaziicola (10)

\section{Genus Passalora \\ Moraceae}

Single species; on Broussonetia.

Passalora broussonetiae (24)

\section{Poaceae}

On Agrostis: Stromata 20-50 $\mu \mathrm{m}$ in diam.; conidiophores $10-52 \times 3-5 \mu \mathrm{m}$, unbranched, not geniculate; conidia 18-38 × 1.5-2 $\mu \mathrm{m}, 3-4$ septate. Passalora fusimaculans (25) On Echinochloa: Stromata 12-14 $\mu \mathrm{m}$ in diam.; conidiophores 20-150 × 3-9 $\mu \mathrm{m}$, unbranched, geniculate; conidia 20-57 × 3-5 $\mu \mathrm{m}, 1-5$ septate...............Passalora barretoana (23) On Panicum; see Passalora fusimaculans

\section{Genus Pseudocercospora Acanthaceae}

Single species; on Justicia ...............Pseudocercospora consociata (31)

\section{Anacardiaceae}

Single species; on Spondias .Pseudocercospora mombin (37)

\section{Araliaceae}

Single species; on Polyscias.

\section{Balsaminaceae}

Single species; on Impatiens ..............Pseudocercospora balsaminae (27)

\section{Bignoniaceae}

On Oroxylum: Stromata 14-21 $\mu \mathrm{m}$ in diam.; conidiophores 11-16 × 3-5 $\mu \mathrm{m}$, unbranched, geniculate; conidia $31-75 \times 2-4 \mu \mathrm{m}, 1-5-$ septate............Pseudocercospora oroxyli (40) On Radermachera: Stromata $8-44 \mu \mathrm{m}$ in diam.; conidiophores 9-26 $\times 3-5 \mu \mathrm{m}$, unbranched, geniculate; conidia 29-38 $\times 2-3$ $\mu \mathrm{m}, 0$-3-septate.

Pseudocercospora radermachericola (45) On Tabebuia: Stromata 20-34 $\mu \mathrm{m}$ in diam.; conidiophores 6-10 × 2-4 $\mu \mathrm{m}$, unbranched, not geniculate; conidia 42-64 × 2-3 $\mu \mathrm{m}, 4-8-$ septate.............Pseudocercospora jahnii (34)

\section{Cannabaceae}

Single species; on Trema. .Pseudocercospora tremicola (49) 


\section{Capparaceae}

Single species; on Crateva. .Pseudocercospora cratevae (32)

\section{Convolvulaceae}

Single species; on Operculina Pseudocercospora timorensis (48)

\section{Dioscoreaceae}

Single species; on Dioscorea.

\section{Euphorbiaceae}

Pseudocercospora carbonacea (29)

On Bischofia: Stromata 5-17 $\mu \mathrm{m}$ in diam.; conidiophores 9-24 × 2-6 $\mu \mathrm{m}$, unbranched, geniculate; conidia 41-56 × 2-3 $\mu \mathrm{m}, 4-6-$ septate.........Pseudocercospora bischofiae (28)

On Mallotus: Stromata $10-40 \mu \mathrm{m}$ in diam.; conidiophores 10-40 × 3-5 $\mu \mathrm{m}$, unbranched, geniculate; conidia 33-75 × 3-4 $\mu \mathrm{m}, 3-7-$ septate......Pseudocercospora malloticola (36) Lythraceae

Single species; on Punica.

Menispermaceae

Single species; on Cyclea. Pseudocercospora cycleae (33)

\section{Moraceae}

Single species; on Morus Pseudocercospora mori (38)

\section{Olacaceae}

Single species; on Olax.

\section{Plantaginaceae}

Pseudocercospora olacicola (39)

Single species; on Scoparia.

.............Pseudocercospora scopariicola (47)

\section{Rosaceae}

Single species; on Rosa

.Pseudocercospora puderi (43)

\section{Rubiaceae}

Single species; on Paederia

\section{Schizaeaceae (fern)}

Pseudocercospora paederiae (41)

Single species; on Lygodium.

\section{Solanaceae}

Single species; on Lycianthes .Pseudocercospora atromarginalis (26)

\section{Thelypteridaceae (Fern)}

Single species; on Christella.....................

\section{Vitaceae}

.Pseudocercospora christellae (30)

Single species; on Vitis

..Pseudocercospora riachueli var. horiana (46)

\section{Genus Zasmidium}

Fabaceae

Single species; on Cassia. .Zasmidium cassiicola (50)

\section{Treatment of cercosporoid fungi}

\section{Treatment of true cercosporoid fungi}

Cercospora arecacearum Hidayat \& Meeboon, Mycol. Progr. 8: 116, 2009. Figs 2-3.

Leaf spots circular, 1-7 mm diam., pale brown to dark brown in the centre, with medium brown to dark brown margin. Colonies amphigenous, scattered, dark brown. Mycelium internal, inconspicuous. Stromata oval to ellipsoidal, substomatal, $10-75 \mu \mathrm{m}$ diam. $(\bar{x}=$ $42.5 \mu \mathrm{m}, \mathrm{n}=9$ ), brown, composed of swollen hyphal cells, subglobose, rounded to angular in outline, $6-13 \mu \mathrm{m}$ wide $(\bar{x}=9 \mu \mathrm{m}, \mathrm{n}=15)$, brown to dark brown, wall $0.5-0.8 \mu \mathrm{m}$ wide $(\bar{x}$ $=0.67 \mu \mathrm{m}, \mathrm{n}=15)$, smooth. Conidiophores fasciculate, arising from stromata (2-22 per fascicle), emerging through stomata, unbranched, straight to curved, cylindrical, 45$117 \times 4-6 \mu \mathrm{m}(\bar{x}=73.6 \times 5 \mu \mathrm{m}, \mathrm{n}=16), 3-5$ septate, distance between septa $8-35 \mu \mathrm{m}(\bar{x}=$ $23.4 \mu \mathrm{m}, \mathrm{n}=25$ ), medium brown, paler at the apex, wall $0.5-0.8 \mu \mathrm{m}$ wide $(\bar{x}=0.58 \mu \mathrm{m}, \mathrm{n}=$ 25), smooth, geniculate. Conidiogenous cells integrated, terminal, cylindrical, $12-35 \times 4-5$ $\mu \mathrm{m}(\bar{x}=24 \times 4.5 \mu \mathrm{m}, \mathrm{n}=7)$, pale brown; conidiogenous loci conspicuous, subcircular, 2-3 $\mu \mathrm{m}$ wide $(\bar{x}=2.6 \mu \mathrm{m}, \mathrm{n}=30)$, dark brown, wall $0.5-0.8 \mu \mathrm{m}$ thick $(\bar{x}=0.6 \mu \mathrm{m}, \mathrm{n}=$ 30). Conidia solitary, acicular, straight to curved, 41-130 × 4-5 $\mu \mathrm{m}(\bar{x}=69 \times 4 \mu \mathrm{m}, \mathrm{n}=$ 5), 3-14-septate, hyaline, thin-walled, smooth, tip acute, base truncate to slighty obconically truncate, hila thickened and darkened, 1.5-3 $\mu \mathrm{m}$ wide $(\bar{x}=2.12 \mu \mathrm{m}, \mathrm{n}=5)$, wall of the hila $0.25-0.3 \mu \mathrm{m}(\bar{x}=0.28 \mu \mathrm{m}, \mathrm{n}=5)$ thick.

Colonies on PDA after 3 weeks at $25^{\circ} \mathrm{C}$ grey, 4.5-5 mm diam., surface ridged. Hyphae smooth, brown, $1-12 \mu \mathrm{m}$ wide $(\bar{x}=4.3 \mu \mathrm{m}, \mathrm{n}$ $=30$ ), septate, constricted at the septa, distance between septa $8-26 \mu \mathrm{m}(\bar{x}=13.08 \mu \mathrm{m}, \mathrm{n}=$ 30 ), wall $0.3-1 \mu \mathrm{m}$ wide $(\bar{x}=0.66 \mu \mathrm{m}, \mathrm{n}=$ 30 ), smooth. Conidia not formed in culture.

Known hosts - Areca catechu L., Areca sp. (Arecaceae). 
Known distribution - Asia: Thailand. Material examined - Chiang Rai Province, Muang District, Sri Pangsang Village, on leaves of Areca sp. (Arecaceae), 15 January 2010, P. Phengsintham (P521), GenBank accession no (ITS, KC677879).

Notes - Morphologically, the present collection is similar to those described by Toanun et al. (2009).

Literature - To-anun et al. (2009: 115121).

Cercospora balsaminiana J.M. Yen \& Lim, Cah. Pacifique 14: 91, 1970. Figs 4-5.

Leaf spots suborbicular to irregular, 1-13 $\mathrm{mm}$ in diam., grey-brown in the centre, with dark brown margin. Colonies amphigenous, scattered, dark brown. Mycelium internal; hyphae branched, $2-3 \mu \mathrm{m}$ wide $(\bar{x}=2.66 \mu \mathrm{m}$, $\mathrm{n}=5)$, septate, constricted at the septa, distance between septa $6-15 \mu \mathrm{m}(\bar{x}=9 \mu \mathrm{m}, \mathrm{n}=5)$, brownish or green-hyaline, wall $0.3-0.5 \mu \mathrm{m}$ wide $(\bar{x}=0.43 \mu \mathrm{m}, \mathrm{n}=5)$, smooth, forming plate-like plectenchymatous stromatic hyphal aggregations. Stromata developed, small to medium-sized, globular to subglobular, substomatal and intraepidermal, 8-36 $\mu \mathrm{m}$ in diam. $(\bar{x}=18.41 \mu \mathrm{m}, \mathrm{n}=7)$, dark brown to black in mass, composed of swollen hyphal cells, subglobose, rounded to angular in outline, $5-10 \mu \mathrm{m}$ wide $(\bar{x}=7.28 \mu \mathrm{m}, \mathrm{n}=20)$, brown to dark brown, wall $0.5-1 \mu \mathrm{m}$ wide $(\bar{x}=$ $0.76 \mu \mathrm{m}, \mathrm{n}=20$ ), smooth. Conidiophores fasciculate, arising from stromata (3-9 per fascicle), emerging through stomata, unbranched, straight to curved, cylindrical, 18-62 $\times 4-6 \mu \mathrm{m}(\bar{x}=49.36 \times 4.99 \mu \mathrm{m}, \mathrm{n}=15), 1-3-$ septate, distance between septa 5-31 $\mu \mathrm{m}(\bar{x}=$ $16.33 \mu \mathrm{m}, \mathrm{n}=20)$, medium brown, paler at the apex, wall $0.5-0.8 \mu \mathrm{m}$ wide $(\bar{x}=0.6 \mu \mathrm{m}, \mathrm{n}=$ 20), smooth, 0-2-times geniculate. Conidiogenous cells terminal, cylindrical, 7-31 × 4-5 $\mu \mathrm{m}(\bar{x}=21.75 \times 4.9 \mu \mathrm{m}, \mathrm{n}=8)$, pale brown; conidiogenous loci conspicuous, subcircular, $2-4 \mu \mathrm{m}$ wide $(\bar{x}=3.41 \mu \mathrm{m}, \mathrm{n}=8)$, wall $0.5-$ $0.8 \mu \mathrm{m}$ thick $(\bar{x}=0.57 \mu \mathrm{m}, \mathrm{n}=8)$, thickened and darkened.

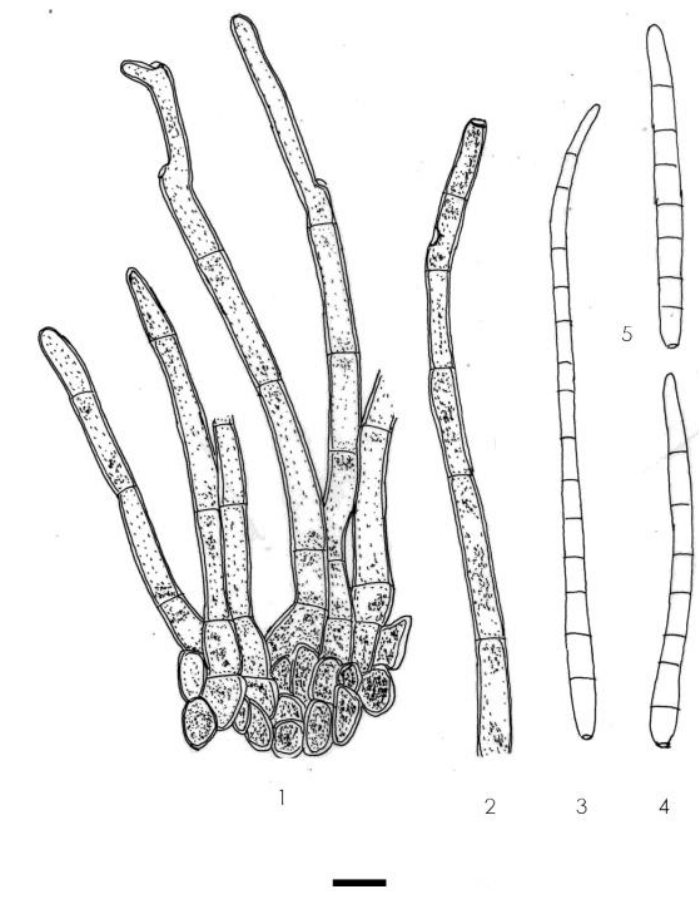

Fig. 2 - Cercospora arecacearum from Areca sp.: 1. Stroma with attached conidiophores. 2. Conidiophore. 3-5. Conidia. Bars: 1-5 =10 $\mu \mathrm{m}$.

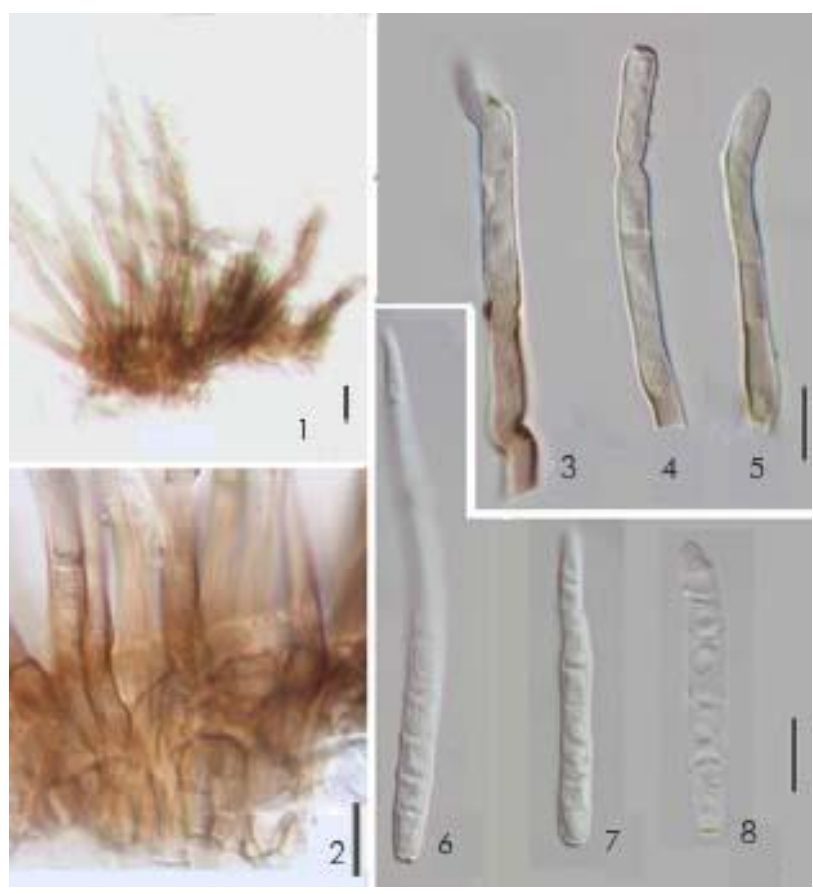

Fig. 3 - Cercospora arecacearum from Areca sp.: 1-2. Stroma with attached conidiophores. 3-5. Conidiophore. 6-8. Conidia. Bars: 1. Not to scale. $2-8=10 \mu \mathrm{m}$. 
Conidia solitary, acicular to obclavate, straight to curved, 45-155 $\times 2-4 \mu \mathrm{m}(\bar{x}=$ $90.63 \times 3.29 \mu \mathrm{m}, \mathrm{n}=13), 5-16$-septate, hyaline to subhyaline, thin-walled, $0.3 \mu \mathrm{m}(\bar{x}=0.3$ $\mu \mathrm{m}, \mathrm{n}=13)$, smooth, tip acute, base obconically truncate; hila thickened and darkened $1-3 \mu \mathrm{m}$ wide $(\bar{x}=1.88 \mu \mathrm{m}, \mathrm{n}=13)$, wall of the hila $0.3-0.5 \mu \mathrm{m}(\bar{x}=0.46 \mu \mathrm{m}, \mathrm{n}=$ 13) thick.

Colonies on PDA after 3 weeks at $25^{\circ} \mathrm{C}$ dark grey mycelium, 20-25 mm diam., hyphae $2-10 \mu \mathrm{m}$ wide $(\bar{x}=6.8 \mu \mathrm{m}, \mathrm{n}=20)$, septate, constricted at the septa, distance between septa 11-20 $\mu \mathrm{m}(\bar{x}=14.4 \mu \mathrm{m}, \mathrm{n}=20)$, brownish or subhyaline, wall $0.3-1 \mu \mathrm{m}$ wide $(\bar{x}=0.72 \mu \mathrm{m}$, $\mathrm{n}=20$ ), smooth. Conidia not formed in culture.

Known hosts - Impatiens balsamina L., I. walleriana Hook. f. (Balsaminaceae)

Known distribution - Asia: China, Thailand.

Material examined - Chiang Rai Province, Maechan District, Doitung National Part, on leaves of Impatiens balsaminiana (Balsaminaceae), 20 July 2010, P. Phengsintham (P603). GenBank accession no (ITS, KC677881); Muang District, Hah Ngaek Pho Khoune Village, on leaves of Impatiens walleriana, 28 July 2010, P. Phengsintham (P608), GenBank accession no (ITS, KC677880; LSU, KC677915).

Notes - The collections differ from the description of $C$. balsaminiana published by To-anun et al. (2011) in having shorter conidia and unbranched conidiophores. To-anun et al. (2011) regarded the Cercospora on Impatiens balsamina as $C$. balsaminiana and that on Impatiens walleriana as C. fukushiana, based on presence of branched conidiophores.

Literature - Yen \& Lim (1980: 155); Crous \& Braun (2003: 75); To-anun et al. (2011: 48).

Cercospora basellae-albae R.K. Srivast., S. Norayana \& A.K. Srivast. Indian Phythopatol. 47: 229, 1994.

Figs 6-7.

Leaf spots circular or suborbicular, 1-5 mm diam., grayish brown to dark brown in the centre, and with brown to dark brown margin.

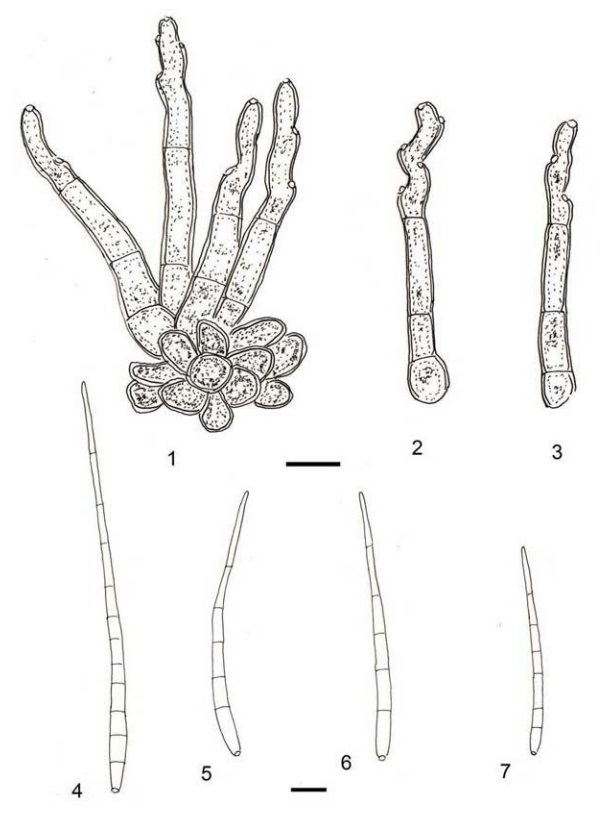

Fig. 4 - Cercospora balsaminiana on Impatiens balsamina from leaf spots: 1 . Stroma with attached conidiophores. 2. Conidiophore. 4-7. Conidia. Bars: 1-7 =10 $\mu \mathrm{m}$.

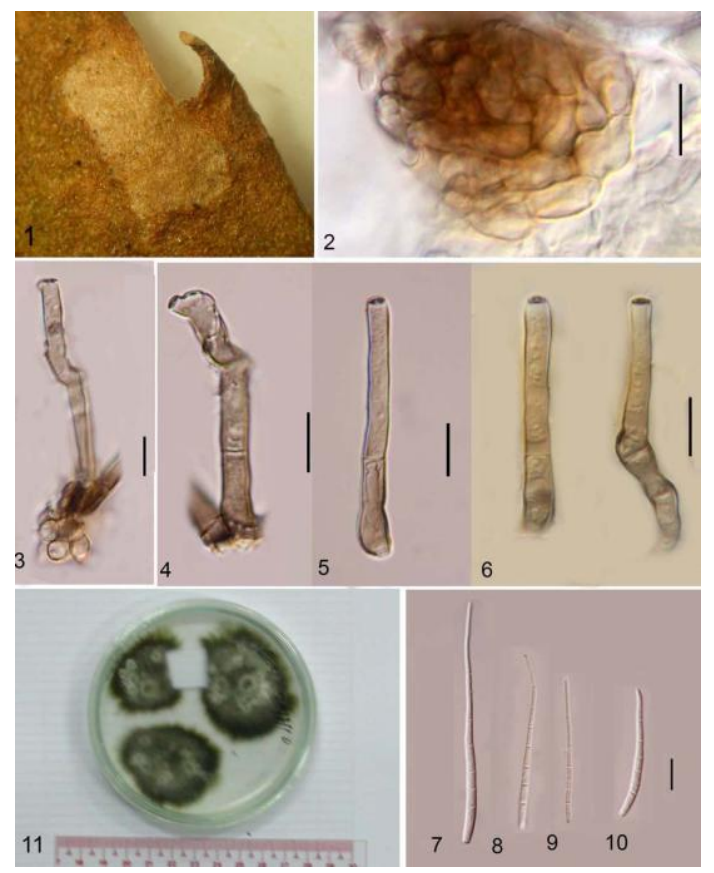

Fig. 5 - Cercospora balsaminiana on Impatiens balsamina from leaf spots: 1. Leaf spot on host leaf (lower surface). 2. Stroma. 3. Stroma with attached conidiophores. 4-6. Conidiophores. 7-10. Conidia. 11. Culture. Bars: 1 . Not to scale. $2-10=10 \mu \mathrm{m} .11=10$ $\mathrm{mm}$. 
Colonies amphigenous, scattered, dark brown. Mycelium internal; hyphae branched, 2-3 $\mu \mathrm{m}$ wide $(\bar{x}=2.6 \mu \mathrm{m}, \mathrm{n}=5)$, septate, constricted at the septa, distance between septa 7-10 $\mu \mathrm{m}(\bar{x}=8.4 \mu \mathrm{m}, \mathrm{n}=5)$, brownish or green-hyaline, wall $0.3-0.5 \mu \mathrm{m}$ wide $(\bar{x}=0.34$ $\mu \mathrm{m}, \mathrm{n}=5$ ), smooth, forming plate-like plectenchymatous stromatic hyphal aggregations. Stromata well-developed, oval to ellipsoidal, substomatal, $25-45 \mu \mathrm{m}$ diam. $(\bar{x}=32.5 \mu \mathrm{m}, \mathrm{n}$ $=4$ ), brown, composed of swollen hyphal cells, subglobose, rounded to angular in outline, 4-10 $\mu \mathrm{m}$ wide $(\bar{x}=6.7 \mu \mathrm{m}, \mathrm{n}=30)$, brown to dark brown, wall $0.8-1 \mu \mathrm{m}$ wide $(\bar{x}=0.81 \mu \mathrm{m}, \mathrm{n}=$ $30)$, smooth. Conidiophores fasciculate, arising from stromata (10-24 per fascicle), emerging through stomata, unbranched, straight to curved, cylindrical, $11-50 \times 3-5 \mu \mathrm{m}(\bar{x}=$ $33.16 \times 4.35 \mu \mathrm{m}, \mathrm{n}=24), 0-2$-septate, distance between septa 5-25 $\mu \mathrm{m}(\bar{x}=12.07 \mu \mathrm{m}, \mathrm{n}=$ 13), medium brown, paler at the apex, wall $0.5-0.8 \mu \mathrm{m}$ wide $(\bar{x}=0.73 \mu \mathrm{m}, \mathrm{n}=13)$, smooth, 0-2 times geniculate, width uniform. Conidiogenous cells integrated, terminal, cylindrical, $11-35 \times 3-5 \mu \mathrm{m}(\bar{x}=19.55 \times 4.11$ $\mu \mathrm{m}, \mathrm{n}=9$ ), pale brown; conidiogenous loci conspicuous, subcircular, $1.5-3 \mu \mathrm{m}$ wide $(\bar{x}=$ $2.36 \mu \mathrm{m}, \mathrm{n}=30$ ), dark brown, wall $0.5-0.8 \mu \mathrm{m}$ thick $(\bar{x}=0.58 \mu \mathrm{m}, \mathrm{n}=30)$. Conidia solitary, acicular, straight to curved, 47-75 $\times 2-4 \mu \mathrm{m}$ $(\bar{x}=60 \times 3 \mu \mathrm{m}, \mathrm{n}=9), 3-7$-septate, hyaline, thin-walled, smooth, tip acute, base truncate or slightly narrowed at the very base, hila thickened and darkened, $1.5-2 \mu \mathrm{m}$ wide $(\bar{x}=$ $1.78 \mu \mathrm{m}, \mathrm{n}=7)$, wall of the hila $0.3-0.5 \mu \mathrm{m}(\bar{x}$ $=0.44 \mu \mathrm{m}, \mathrm{n}=7$ ) thick.

Known hosts - Basella alba L., Basella rubra L. (Basellaceae).

Known distribution - Asia: India, Laos, Thailand.

Material examined - Chiang Rai Province, Muang District, Sri Pangsang Village, on leaves of Basella rubra (Basella-ceae), 10 August 2009, P. Phengsintham (P433).

Notes - A true Cercospora s. str. close to or identical with $C$. apii s. lat. (Crous \& Braun, 2003). The present collection agrees well with the description of $C$. basellae-albae given by To-anun et al. (2011) in having a similar size of conidiophores and conidia [conidiophores 25-70 × 3-6 $\mu \mathrm{m}$ and conidia $17-93 \times 3-5 \mu \mathrm{m}]$.
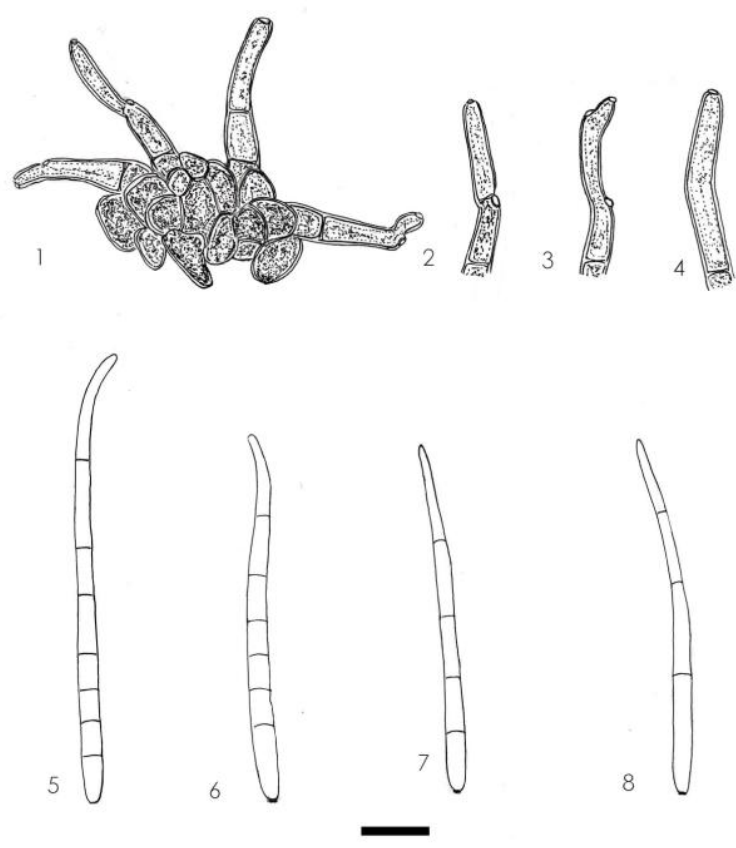

Fig. 6 - Cercospora basellae-albae on Basella rubra: 1. Stroma with attached conidiophores. 2-4. Conidiophores. 5-8. Conidia. Bars: 1-8 = $10 \mu \mathrm{m}$.

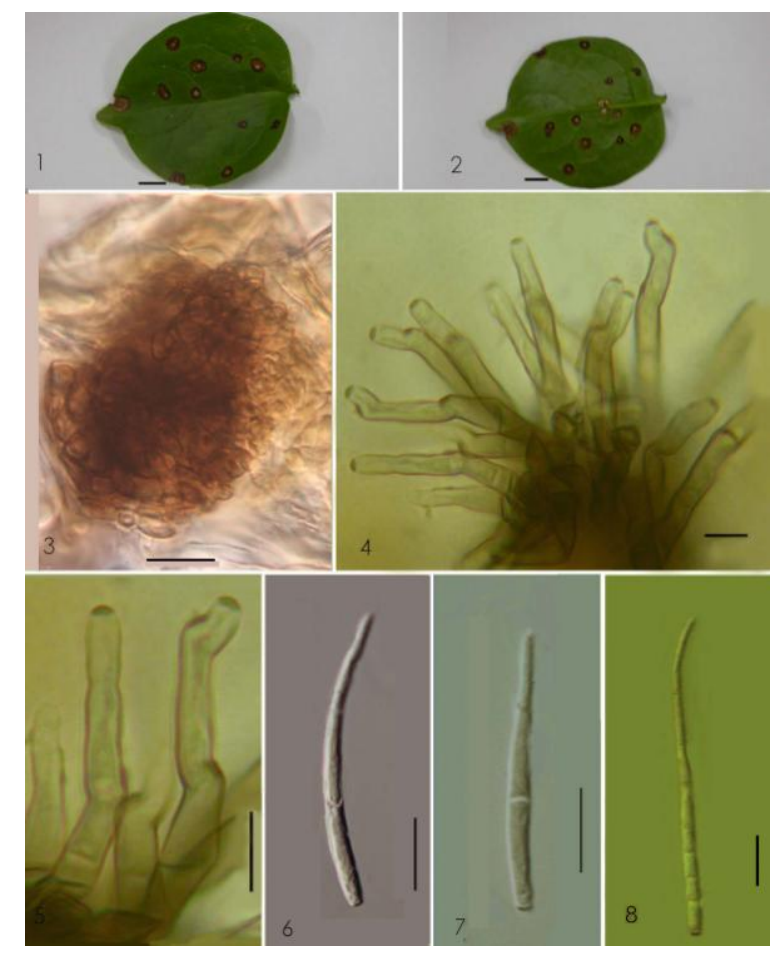

Fig. 7 - Cercospora basellae-albae on Basella rubra from leaf spots: 1. Leaf spots on host leaves (1. upper surface, 2. lower surface). 34. Stromata with attached conidiophores. 5. Conidiophore. 6-8. Conidia. Bars: $1-2=10$ $\mathrm{mm}, 3-8=10 \mu \mathrm{m}$. 
Literature - Crous \& Braun (2003); Toanun et al. (2011: 50).

Cercospora broussonetiae Y.L. Guo \& L. Xu, Mycosystema 21: 181. 2002.

Figs 8-9.

Leaf spots small to fairly large, suborbicular to irregular, 1-7 $\mathrm{mm}$ in diam., grey-brown in the centre, and with dark brown violet margin. Colonies amphigenous, scattered, dark brown. Mycelium internal; hyphae branched, $2-8 \mu \mathrm{m}$ wide $(\bar{x}=5.66 \mu \mathrm{m}$, $\mathrm{n}=13)$, septate, constricted at the septa, distance between septa 5-10 $\mu \mathrm{m}(\bar{x}=6.66 \mu \mathrm{m}$, $\mathrm{n}=13$ ), brownish or green-hyaline, wall $0.3-$ $0.5 \mu \mathrm{m}$ wide $(\bar{x}=0.43 \mu \mathrm{m}, \mathrm{n}=13)$, smooth, forming plate-like plectenchymatous stromatic hyphal aggregations. Stromata developed, small to medium-sized, globular to subglobular, substomatal and intraepidermal, 12$24 \mu \mathrm{m}$ in diam. ( $\bar{x}=19.6 \mu \mathrm{m}, \mathrm{n}=5)$, dark brown to black in mass, composed of swollen hyphal cells, subglobose, rounded to angular in outline, 5-8 $\mu \mathrm{m}$ wide $(\bar{x}=6.2 \mu \mathrm{m}, \mathrm{n}=30)$, brown to dark brown, wall $0.5-0.8 \mu \mathrm{m}$ wide $(\bar{x}$ $=0.62 \mu \mathrm{m}, \mathrm{n}=30$ ), smooth. Conidiophores solitary or fasciculate, arising from stromata (2-7 per fascicle), emerging through stomata, unbranched, straight to curved, cylindrical, 30$140 \times 5-6 \mu \mathrm{m}(\bar{x}=63.7 \times 5.67 \mu \mathrm{m}, \mathrm{n}=13)$, 1-6-septate, distance between septa 10-46 $\mu \mathrm{m}$ $(\bar{x}=23.3 \mu \mathrm{m}, \mathrm{n}=30)$, medium brown, paler at the apex, wall $0.5-0.8 \mu \mathrm{m}$ wide $(\bar{x}=0.58 \mu \mathrm{m}$, $\mathrm{n}=30$ ), smooth, 0-1-times geniculate. Conidiogenous cells terminal, cylindrical, 20$46 \times 5-6 \mu \mathrm{m}(\bar{x}=30.7 \times 5.33 \mu \mathrm{m}, \mathrm{n}=7)$, pale brown; conidiogenous loci conspicuous, subcircular, $3-4 \mu \mathrm{m}$ wide $(\bar{x}=3.33 \mu \mathrm{m}, \mathrm{n}=7)$, wall $0.5-0.8 \mu \mathrm{m}$ thick $(\bar{x}=0.6 \mu \mathrm{m}, \mathrm{n}=7)$, thickened and darkened. Conidia solitary, acicular to obclavate, straight to curved, 78$252 \times 2.5-4 \mu \mathrm{m}(\bar{x}=140.5 \times 2.12 \mu \mathrm{m}, \mathrm{n}=7)$, 2-16-septate, hyaline to subhyaline, thinwalled $0.3-0.5 \mu \mathrm{m}(\bar{x}=0.35 \mu \mathrm{m}, \mathrm{n}=7)$, smooth, tip acute, base truncate; hila thickened and darkened $2-3 \mu \mathrm{m}$ wide $(\bar{x}=2.62 \mu \mathrm{m}, \mathrm{n}=$ $7)$, wall of the hila $0.3-0.5 \mu \mathrm{m}(\bar{x}=0.35 \mu \mathrm{m}, \mathrm{n}$ $=7$ ) thick.

Known hosts - Broussonetia kaempferi Siebold, B. papyrifera (L.) L'Hér. ex Vent. (Moraceae).
Thailand.

Known distribution - Asia: China,

Material examined - Chiang Rai Province, Maechan District, Doitung National Park, on leaves of Broussonetia papyrifera (Moraceae), 4 December 2009, P. Phengsintham (P472); Chiang Rai Province, Muang District, Bandu Village, on leaves of Broussonetia papyrifera (Moraceae), 30 July 2010, P. Phengsintham (P610, GenBank accession no (ITS, KC677882; LSU, KC677916) ).

Notes - A true Cercospora s. str. close to or identical with Cercospora apii s. lat. (Crous \& Braun, 2003).

Literature - Crous \& Braun (2003: 89).

Cercospora buteae Munjal, Lall \& Chona, Indian Phythopathol. 12: 133, (1959) 1960. Figs 10-11.

Leaf spots small to fairly large, suborbicular to irregular, 5-15 $\mathrm{mm}$ in diam., grey-brown in the centre, and dark brown margin. Colonies hypophyllous, scattered, dark brown. Mycelium internal, inconspicuous. Stromata developed, small to medium-sized, globular to subglobular, substomatal and intraepidermal, $18-25 \mu \mathrm{m}$ in diam. $(\bar{x}=21.5$ $\mu \mathrm{m}, \mathrm{n}=4)$, dark brown to black in mass, composed of swollen hyphal cells, subglobose, rounded to angular in outline, $4-10 \mu \mathrm{m}$ wide $(\bar{x}=7.8 \mu \mathrm{m}, \mathrm{n}=13$ ), brown to dark brown, wall $0.5-0.8 \mu \mathrm{m}$ wide $(\bar{x}=0.65 \mu \mathrm{m}, \mathrm{n}=13)$, smooth. Conidiophores fasciculate, arising from stromata (1-7 per fascicle), emerging through stomata, unbranched, straight to curved, cylindrical, 56-235 × 5-6 $\mu \mathrm{m}(\bar{x}=134$ $\times 5.67 \mu \mathrm{m}, \mathrm{n}=7), 4-10$-septate, distance between septa $12-40 \mu \mathrm{m}(\bar{x}=25.5 \mu \mathrm{m}, \mathrm{n}=$ 30 ), medium brown, paler at the apex, wall $0.5-1 \mu \mathrm{m}$ wide $(\bar{x}=0.59 \mu \mathrm{m}, \mathrm{n}=30)$, smooth, 0-2-times geniculate. Conidiogenous cells terminal, cylindrical, $12-24 \times 4-5 \mu \mathrm{m}(\bar{x}=$ $18.5 \times 4.5 \mu \mathrm{m}, \mathrm{n}=7$ ), pale brown; conidiogenous loci conspicuous, subcircular, 2-3 $\mu \mathrm{m}$ wide $(\bar{x}=2.75 \mu \mathrm{m}, \mathrm{n}=7)$, wall $0.5-$ $0.8 \mu \mathrm{m}$ thick $(\bar{x}=0.57 \mu \mathrm{m}, \mathrm{n}=7)$, thickened and darkened. Conidia solitary, acicular to obclavate, straight to curved, $60-110 \times 2-4 \mu \mathrm{m}$ $(\bar{x}=77.33 \times 3 \mu \mathrm{m}, \mathrm{n}=5), 4-5$-septate, hyaline to subhyaline, thin-walled $0.25-0.3 \mu \mathrm{m}(\bar{x}=$ $0.283 \mu \mathrm{m}, \mathrm{n}=5)$, smooth, tip acute, 


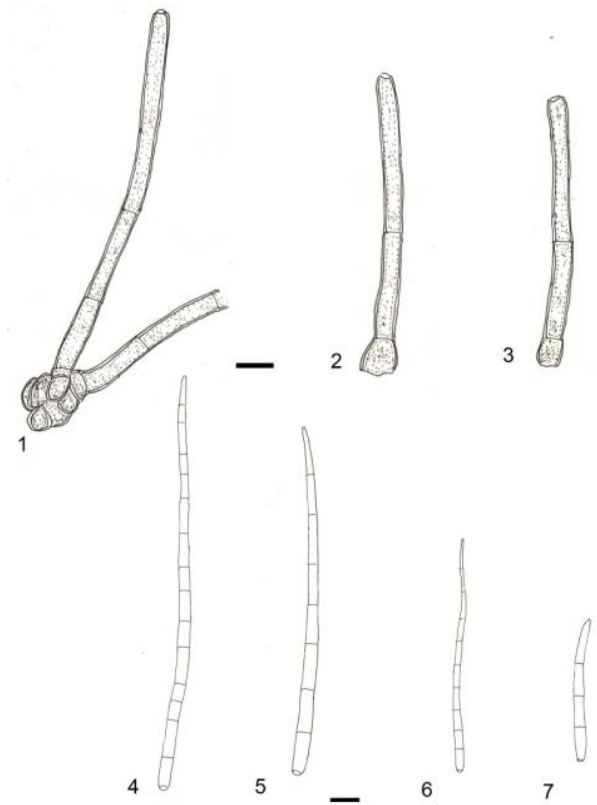

Fig. 8 - Cercospora broussonetiae on Broussonetia papyrifera from leaf spots: 1 . Stroma with attached conidiophores. 2-3. Conidiophores. 4-7. Conidia. Bars: $1-7=10$ $\mu \mathrm{m}$.

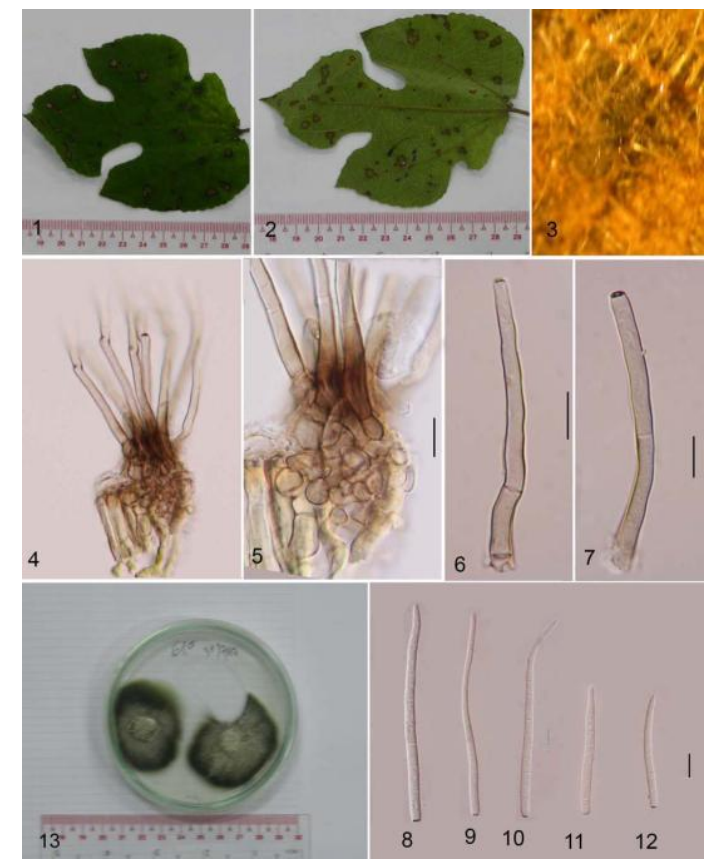

Fig. 9 - Cercospora broussonetiae on Broussonetia papyrifera from leaf spots: 1-2. Leaf spots on host leaves (1. upper surface, 2. lower surface). 3. Colonies. 4-5. Stromata with attached conidiophores. 6-7. Conidiophores. 8-12. Conidia. 13. Culture. Bars: $1-2=10$ mm. 3. Not to scale. $3-12=10 \mu \mathrm{m} .13 .=10$ $\mathrm{mm}$.

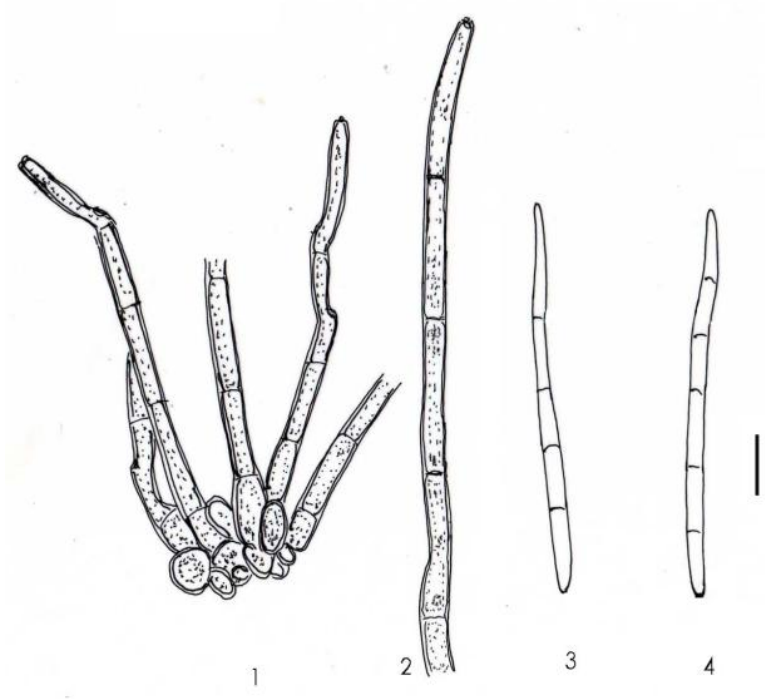

Fig. 10 - Cercospora buteae on Butea monosperma from leaf spots: 1 . Stroma with attached conidiophores. 2. Conidiophore. 3-4. Conidia. Bars: $1-4=10 \mu \mathrm{m}$.

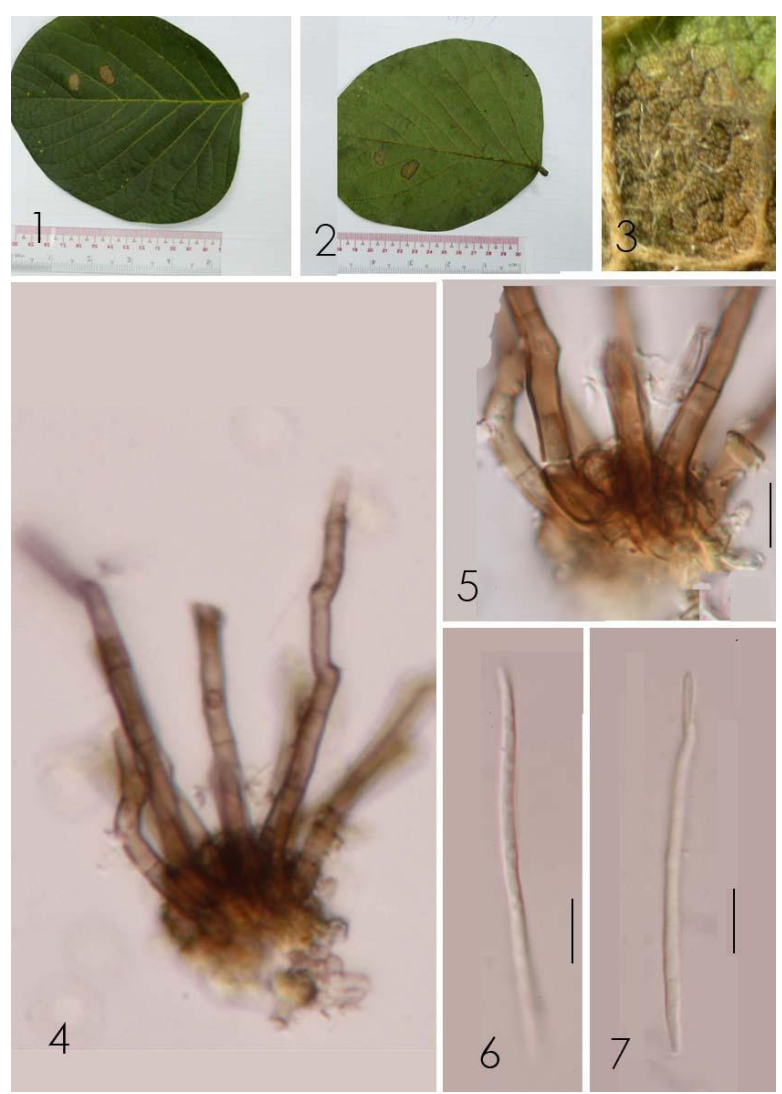

Fig. 11 - Cercospora buteae on Butea monosperma from leaf spots: 1-2. Leaf spots on host leave (1. upper surface, 2. lower surface). 3. Colonies. 4-5. Stromata with attached conidiophores. 6-7. Conidia. Bars: $1-2=10 \mathrm{~mm}$. 3. Not to scale. $4-7=10 \mu \mathrm{m}$. 
base truncate to obconically truncate; hila thickened and darkened $1-2 \mu \mathrm{m}$ wide $(\bar{x}=1.5$ $\mu \mathrm{m}, \mathrm{n}=5)$, wall of the hila $0.3-0.5 \mu \mathrm{m}(\bar{x}=$ $0.4 \mu \mathrm{m}, \mathrm{n}=5$ ) thick.

Known hosts - Butea frondosa K.D. Koenig ex Roxb., B. monosperma Kuntze (Fabaceae).

Known distribution - Asia: India, Thailand.

Material examined - Chiang Rai Province, Muang District, Sri Pangsang Village, on leaves of Butea monosperma (Fabaceae), 22 December 2009, P. Phengsintham (P493).

Notes - The collection from Thailand agrees with the original description of Cercospora buteae by Munjal, Lall \& Chona (1960).

Literature - Crous \& Braun (2003: 91).

Cercospora canescens Ellis \& G. Martin, Amer. Naturalist 16: 1003, 1882. Figs 12-13. $\equiv$ Cercosporiopsis canescens (Ellis \& G. Martin) Miura, Flora of Manchuria and East Mongolia 3: 529, 1928.

$=$ Cercospora vignicaulis Tehon, Mycologia 29: 436, 1937.

Leaf spots small to fairly large, suborbicular to irregular, 3-14 $\mathrm{mm}$ in diam., greybrown in the centre, and with dark brown margin. Colonies amphigenous, scattered, dark brown. Mycelium internal; hyphae branched, 2-4 $\mu \mathrm{m}$ wide $(\bar{x}=3 \mu \mathrm{m}, \mathrm{n}=8)$, septate, constricted at the septa, distance between septa 6-10 $\mu \mathrm{m}(\bar{x}=8 \mu \mathrm{m}, \mathrm{n}=8)$, brownish or green-hyaline, wall $0.3-0.5 \mu \mathrm{m}$ wide $(\bar{x}=0.4$ $\mu \mathrm{m}, \mathrm{n}=8$ ), smooth, forming plate-like plectenchymatous stromatic hyphal aggregations. Stromata developed, small to mediumsized, globular to subglobular, substomatal and intraepidermal, $10-38 \mu \mathrm{m}$ in diam. $(\bar{x}=20$ $\mu \mathrm{m}, \mathrm{n}=7$ ), dark brown to black in mass, composed of swollen hyphal cells, subglobose, rounded to angular in outline, 5-11 $\mu \mathrm{m}$ wide ( $\bar{x}=7.6 \mu \mathrm{m}, \mathrm{n}=30$ ), brown to dark brown, wall $0.5-0.8 \mu \mathrm{m}$ wide $(\bar{x}=0.65 \mu \mathrm{m}, \mathrm{n}=30)$, smooth. Conidiophores fasciculate or solitary, arising from stromata (1-7 per fascicle), emerging through stomata, unbranched, straight to curved, cylindrical, 28-
$78 \times 4-6 \mu \mathrm{m}(\bar{x}=51.17 \times 5.1 \mu \mathrm{m}, \mathrm{n}=13), 0$ 2-septate, distance between septa 8-35 $\mu \mathrm{m}(\bar{x}$ $=22.35 \mu \mathrm{m}, \mathrm{n}=30$ ), medium brown, paler at the apex, wall $0.5-0.8 \mu \mathrm{m}$ wide $(\bar{x}=0.69 \mu \mathrm{m}$, $\mathrm{n}=30$ ), smooth, not geniculate. Conidiogenous cells terminal, cylindrical, $6-35 \times 4-6 \mu \mathrm{m}(\bar{x}=$ $23.8 \times 5.1 \mu \mathrm{m}, \mathrm{n}=8$ ), pale brown; conidiogenous loci conspicuous, subcircular, $2-5 \mu \mathrm{m}$ wide $(\bar{x}=3.14 \mu \mathrm{m}, \mathrm{n}=8)$, wall $0.5-0.8 \mu \mathrm{m}$ thick ( $\bar{x}=0.57 \mu \mathrm{m}, \mathrm{n}=8$ ), thickened and darkened. Conidia solitary, acicular to obclavate, straight to curved, $42-165 \times 3-4 \mu \mathrm{m}$ $(\bar{x}=68.94 \times 3.09 \mu \mathrm{m}, \mathrm{n}=9), 3-14$-septate, hyaline to subhyaline, thin-walled $0.3 \mu \mathrm{m}(\bar{x}=$ $0.3 \mu \mathrm{m}, \mathrm{n}=5$ ), smooth, tip acute, base truncate to somewhat obconically truncate; hila thickened and darkened $2-3 \mu \mathrm{m}$ wide $(\bar{x}=2.5$ $\mu \mathrm{m}, \mathrm{n}=5)$, wall of the hila $0.3-0.5 \mu \mathrm{m}(\bar{x}=$ $0.46 \mu \mathrm{m}, \mathrm{n}=5$ ) thick.

Known hosts - Bauhinia, Boerhavia, Cajanus, Calopogonium, Canavalia, Cassia, Clitoria, Codariocalyx, Crotalaria, Cyamopsis, Desmodium, Erythrina, Flemingia, Gliricidia, Glycine, Heylandia, Indigofera, Kotschya, Lablab, Vigna, Lespedeza, Lathyrus, Lotononis, Lupinus, Macroptilium, Macrotyloma, Medicago, Mimosa, Mucuna, Neonotonia, Phaseolus, Pisum, Psophocarpus, Psoralea, Pterocarpus, Pueraria, Rhynchosia, Senna, Shuteria, Stylosanthes, Teramnus, Vicia, Vigna, Voandzeia (Fabaceae).

Known distribution - Africa: Ghana, Kenya, Malawi, Mauritius, Nigeria, Senegal, Sierra Leone, Somalia, South Africa, Sudan, Tanzania, Togo, Uganda, Zambia, Zimbabwe; Asia: Bangladesh, Brunei, Cambodia, China, Hong Kong, India, Indonesia, Iran, Japan, Korea, Laos, Malaysia, Myanmar, Nepal, Pakistan, Philippines, Tajikistan, Taiwan, Thailand, Uzbekistan; Europe: Georgia, Russia; North America and West Indies: Barbados, Costa Rica, Cuba, Dominican Rep., Haiti, Panama, St. Vincent and the Grenadines, Trinidad and Tobago, USA (AL, FL,HI, LA, IL, KS, MD, MO, MS, NC, SC, TX, VA, WV); Australia; Oceania: Fiji, New Caledonia, New Zealand, Papua New Guinea, Puerto Rico, Solomon Islands, Virgin Islands, Vanuatu; South America: Bolivia, Brazil, Colombia, Ecuador, Guyana, Peru, Venezuela. 


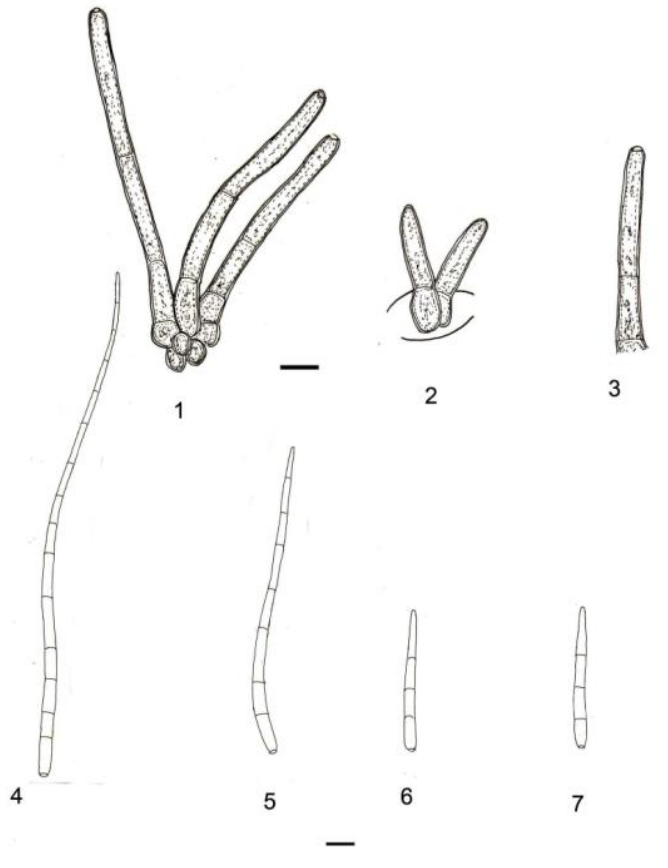

Fig. 12 - Cercospora canescens on Clitoria mariana from leaf spots: 1-2. Stromata with attached conidiophores. 3. Conidiophore. 4-7. Conidia. Bars: $1-7=$ $10 \mu \mathrm{m}$.

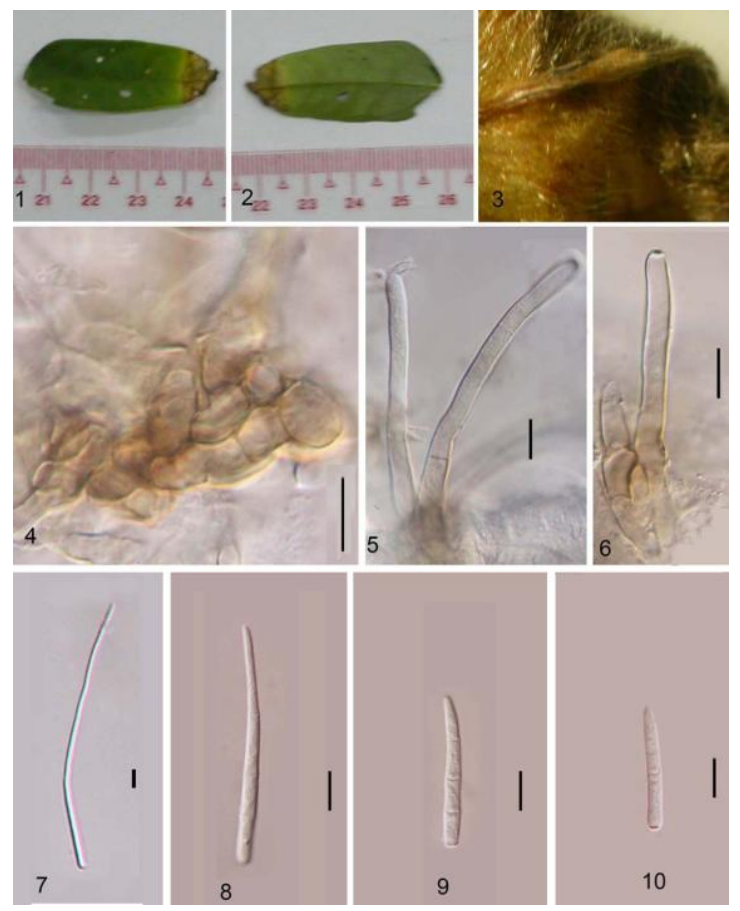

Fig. 13 - Cercospora canescens on Clitoria mariana from leaf spots: 1-2. Leaf spots on host leaves (1. upper surface. 2. lower surface). 3. Colonies. 4. Stroma. 5-6. Stromata with attached conidiophores. 7-10. Conidia. Bars: $1-2=10 \mathrm{~mm}$. 3. Not to scale. $4-10=10 \mu \mathrm{m}$.
Material examined - Chiang Rai Province, Doi Tung National Park, on leaves of Vigna uncuiculata (Fabaceae), 22 August 2009, P. Phengsintham (P441); Chiang Rai Province, Muang District, Sri Pangsang Village, on leaves of Clitoria mariana, 4 August 2010, P. Phengsintham (P612). GenBank accession no (ITS, KC677883; LSU, KC677917).

Notes - The size of conidiophores and conidia is similar to those of $C$. canescens as described by Chupp (1954) and Hsieh \& Goh (1990) [conidiophores 20-200 × 3-6.5 $\mu \mathrm{m}$ and conidia $25-300 \times 2.5-5.5 \mu \mathrm{m}]$.

Literature - Chupp (1954: 288); Vasudeva (1963: 62); Ellis (1976: 264); Hsieh \& Goh (1990: 164); Crous \& Braun (2003: 99).

Cercospora codiaei Gonz. Frag. \& Cif., Rep. Dominic. Est. Agron. Haina, D, Bot. 5: 9, 1926.

Figs 14-15.

Leaf spots suborbicular to irregular, 1-5 $\mathrm{mm}$ in diam., grey-brown in the centre, and with reddish margin. Colonies amphigenous, scattered, dark brown. Mycelium internal, inconspicuous. Stromata developed, small to medium-sized, globular to subglobular, substomatal and intraepidermal, 17-27 $\mu \mathrm{m}$ in diam. $(\bar{x}=21.3 \mu \mathrm{m}, \mathrm{n}=5)$, dark brown to black in mass, composed of swollen hyphal cells, subglobose, rounded to angular in outline, 5-9 $\mu \mathrm{m}$ wide $(\bar{x}=6.7 \mu \mathrm{m}, \mathrm{n}=15)$, brown to dark brown, wall $0.5-0.8 \mu \mathrm{m}$ wide $(\bar{x}$ $=0.6 \mu \mathrm{m}, \mathrm{n}=15$ ), smooth. Conidiophores fasciculate or solitary, arising from stromata (2-10 per fascicle), emerging through stomata, unbranched, straight to curved, cylindrical, 16$49 \times 3-5 \mu \mathrm{m}(\bar{x}=33.8 \times 3.75 \mu \mathrm{m}, \mathrm{n}=15), 1-$ 3-septate, distance between septa 5-18 $\mu \mathrm{m}(\bar{x}$ $=11.5 \mu \mathrm{m}, \mathrm{n}=30$ ), medium brown, paler at the apex, wall $0.5-0.8 \mu \mathrm{m}$ wide $(\bar{x}=0.66 \mu \mathrm{m}, \mathrm{n}=$ 30 ), smooth, not geniculate. Conidiogenous cells terminal, cylindrical, $13-18 \times 3-5 \mu \mathrm{m}(\bar{x}$ $=16 \times 3.67 \mu \mathrm{m}, \mathrm{n}=5)$, pale brown; conidiogenous loci conspicuous, subcircular, $1.5-2 \mu \mathrm{m}$ wide $(\bar{x}=1.83 \mu \mathrm{m}, \mathrm{n}=5)$, wall $0.5-$ $0.8 \mu \mathrm{m}$ thick $(\bar{x}=0.6 \mu \mathrm{m}, \mathrm{n}=5)$, thickened and darkened. Conidia solitary, acicular to obclavate, straight to curved, 50-150 × 2.5-4 $\mu \mathrm{m}(\bar{x}=84 \times 3.16 \mu \mathrm{m}, \mathrm{n}=9), 3-8$-septate, hyaline to subhyaline, thin-walled $0.25-0.3 \mu \mathrm{m}$ $(\bar{x}=0.28 \mu \mathrm{m}, \mathrm{n}=9)$, smooth, tip acute, base 
truncate to somewhat obconically truncate; hila thickened and darkened 1-2 $\mu \mathrm{m}$ wide $(\bar{x}=$ $1.33 \mu \mathrm{m}, \mathrm{n}=9$ ), wall of the hila $0.25-0.3 \mu \mathrm{m}$ $(\bar{x}=0.28 \mu \mathrm{m}, \mathrm{n}=9)$ thick.

Known hosts - Cordiaeum variegatum (L.) A. Juss. (Euphorbiaceae).

Known distribution - Africa: Nigeria; Asia: Thailand; North America and West Indies: Cuba, Dominican Rep.

Material examined - Chiang Mai Province, Maeteng District, Phadeng Village, on leaves of Cordiaeum variegatum (Euphorbiaceae), 7 June 2011, P. Phengsintham (P639).

Notes - The collection agrees well with C. cordiaei as circumscribed by To-anun et al. (2011) [conidiophores 56-213 × 4-5.5 $\mu \mathrm{m}$, slightly geniculate; conidia $29-160 \times 3-4.5$ $\mu \mathrm{m}$, acicular, rarely obclavate, 3-10-septate, truncate at the base; on Codiaeum].

Literature - Chupp (1954: 215); Crous \& Braun (2003: 130); To-anun et al. (2011: 59).

Cercospora coffeicola Berk. \& Cooke, Grevillea 9: 99, $1881 . \quad$ Figs 16-17. = Cercospora coffeae Zimm., Ber. Land- Forstw. Deutsch-Ostafr. 2: 35, 1904.

$=$ Cercospora herrerana Farneti, Atti Ist. Bot. Univ. Pavia, Ser. 2, 9: 37, 1911.

Teleomorph: Mycosphaerella coffeicola (Cooke) J.A. Stev. \& Wellman, J. Wash. Acad. Sci. 34: 262, 1944.

$\equiv$ Sphaerella coffeicola Cooke, Grevillea 9: 11, 1880.

$\equiv$ Mycosphaerella coffeicola (Cooke) Cif., Ist. Bot. Reale Univ. Reale Lab. Crittog. Pavia Atti, Ser. 5, 19: 118, 1962 (comb. superfl.).

Leaf spots small to fairly large, suborbicular to irregular, $1-10 \mathrm{~mm}$ in diam., at first yellowish, later becoming grey-brown in the centre, and with dark brown margin. Colonies amphigenous scattered, dark brown. Mycelium internal, inconspicuous. Stromata developed, small to medium-sized, globular to subglobular, substomatal and intraepidermal, 40-45 $\mu \mathrm{m}$ in diam. $(\bar{x}=42.5 \mu \mathrm{m}, \mathrm{n}=5)$, dark brown to black in mass, composed of swollen hyphal cells, subglobose, rounded to angular in outline, $4-7 \mu \mathrm{m}$ wide $(\bar{x}=5.9 \mu \mathrm{m}, \mathrm{n}=30)$, brown to dark brown, wall $0.5-0.8 \mu \mathrm{m}$ wide $(\bar{x}$ $=0.73 \mu \mathrm{m}, \mathrm{n}=30$ ), smooth.
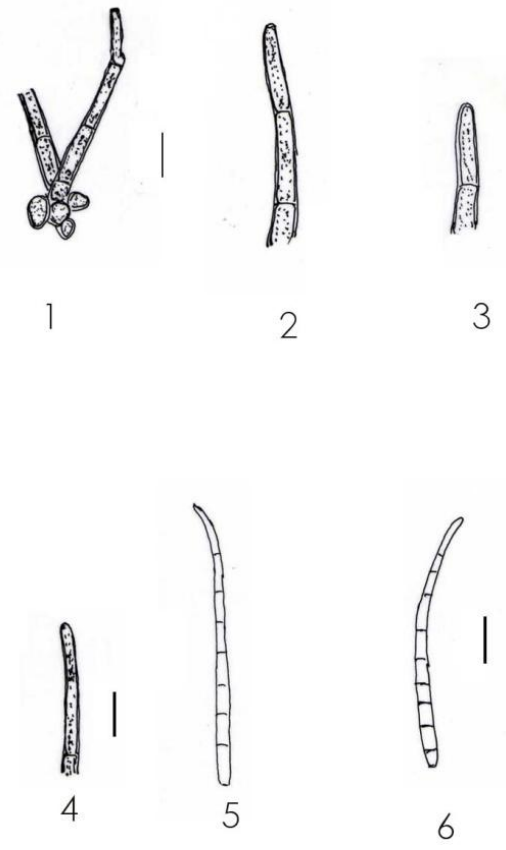

Fig. 14 - Cercospora cordiaei on Cordiaeum variega from leaf spots: 1. Stroma with attached conidiophores. 2-4. Conidiophores. 5-6. Conidia. Bars: $1-6=10 \mu \mathrm{m}$.

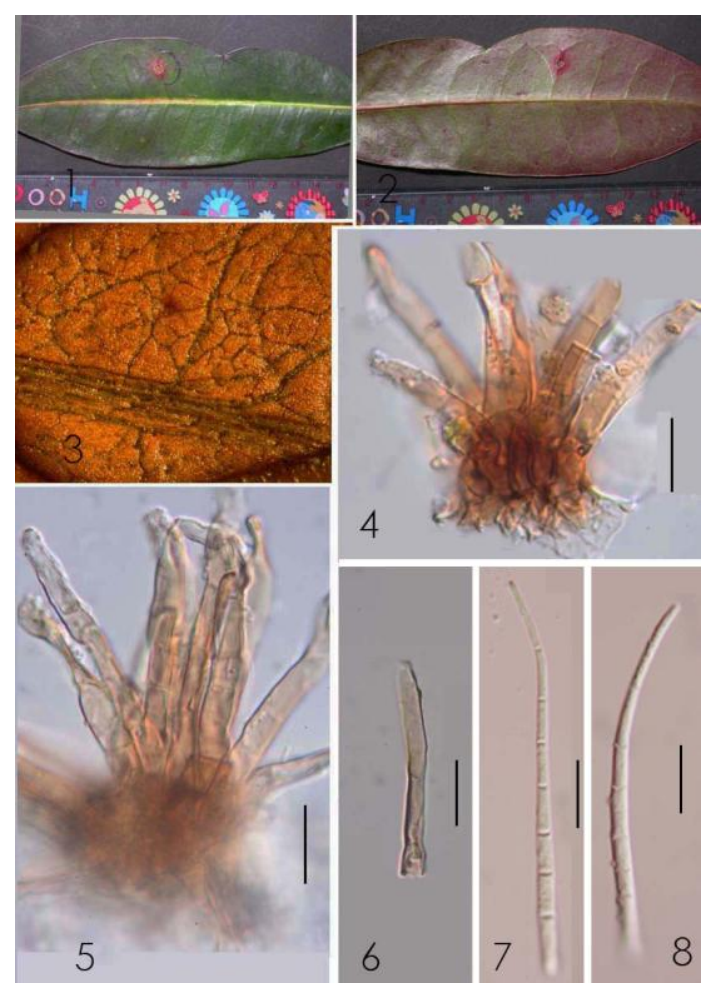

Fig. 15 - Cercospora cordiaei on Cordiaeum variega from leaf spots: 1-2. Leaf spots on host leaves (1. upper surface, 2. lower surface). 3. Colonies. 4-5 Stromata with attached conidiophores. 6. Conidiophore. 7-8. Conidia. Bars: $1-2=10 \mathrm{~mm}$. 3. Not to scale. $4-8=10$ $\mu \mathrm{m}$. 
Conidiophores fasciculate, arising from stromata (2-21 per fascicle), emerging through stomata, unbranched, straight to curved, cylindrical, $9-55 \times 4-5 \mu \mathrm{m}(\bar{x}=52.8 \times 4.46$ $\mu \mathrm{m}, \mathrm{n}=30), 0-5$-septate, distance between septa 9-34 $\mu \mathrm{m}(\bar{x}=18 \mu \mathrm{m}, \mathrm{n}=30)$, medium brown, paler at the apex, wall $0.5-0.8 \mu \mathrm{m}$ wide ( $\bar{x}=0.62 \mu \mathrm{m}, \mathrm{n}=30$ ), smooth, 0-2-times geniculate. Conidiogenous cells integrated, terminal, cylindrical, 9-34 $\times 4-5 \mu \mathrm{m}(\bar{x}=18.8$ $\times 4.6 \mu \mathrm{m}, \mathrm{n}=11$ ), pale brown; conidiogenous loci conspicuous, subcircular, $2-4 \mu \mathrm{m}$ wide $(\bar{x}$ $=3 \mu \mathrm{m}, \mathrm{n}=11)$, wall $0.5-0.8 \mu \mathrm{m}$ thick $(\bar{x}=$ $0.62 \mu \mathrm{m}, \mathrm{n}=11)$, thickened and darkened. Conidia solitary, acicular to obclavate, straight to curved, $54-87 \times 2-3.5 \mu \mathrm{m}(\bar{x}=70.7 \times 2.87$ $\mu \mathrm{m}, \mathrm{n}=7$ ), 6-9-septate, hyaline to subhyaline, thin-walled $0.3-0.5 \mu \mathrm{m}(\bar{x}=0.4 \mu \mathrm{m}, \mathrm{n}=7)$, smooth; tip acute; base truncate to obconically truncate, hila thickened and darkened 1-2 $\mu \mathrm{m}$ wide $(\bar{x}=1.37 \mu \mathrm{m}, \mathrm{n}=7)$, wall of the hila $0.3-0.5 \mu \mathrm{m}(\bar{x}=0.45 \mu \mathrm{m}, \mathrm{n}=7)$ thick.

Known hosts - Coffea arabica L., $C$. canephora Pierre ex A. Froehn, C. excelsa A. Chev., C. laurina Poir., C. liberica W. Bull ex Hiern, C. robusta L. Linden, C. stenophylla $\mathrm{G}$. Don, Coffea spp. (Rubiaceae).

Known distribution - Africa: Angola, Congo, Ethiopia, Gabon, Ghana, Ivory Coast, Kenya, Madagascar, Malawi, Mauritius, Mozambique, Nigeria, Sierra Leone, Somalia, South Africa, Sudan, Tanzania, Togo, Uganda, Zimbabwe; Asia: Brunei, Cambodia, China, India, Indonesia, Japan, Laos, Myanmar, Nepal, Papua New Guinea, Philippines, Thailand, Yemen; North America and West Indies: Costa Rica, Cuba, Dominican Republ., El Salvador, Guadeloupe, Guatemala, Haiti, Jamaica, Martinique, Panama, Puerto Rico, Trinidad and Tobago, USA (FL, HI); Australia; Oceania: American Samoa, Fiji, French Polynesia, Micronesia, New Caledonia, Samoa, Vanuatu; South America: Brazil, Colombia, French Guiana, Guyana, Peru, Suriname, Venezuela.

Material examined - Chiang Mai Province, Maeteng District, Phadeng Village, Garden, on leaves of Coffea arabica (Rubiaceae), 10 September 2007, P. Phengsintham (P301); Chiang Rai Province, Maechan District, Doi Tung National Park, Garden, on leaves of Coffea arabica
(Rubiaceae), 4 December 2009, P. Phengsintham (P481).

Notes - The collections from Thailand agree well with the description of Cercospora coffeicola in Hsieh \& Goh (1990) [conidiophores $20-200 \times 4-6 \mu \mathrm{m}$ and conidia $40-150 \times$ $2-4 \mu \mathrm{m}]$. The variation in the size of conidiophores and conidia ranging from rather short to long are connected with different climate conditions.

Literature - Chupp (1954: 493); Vasudeva (1963: 86); Ellis (1976: 283); Hsieh \& Goh (1990: 285).

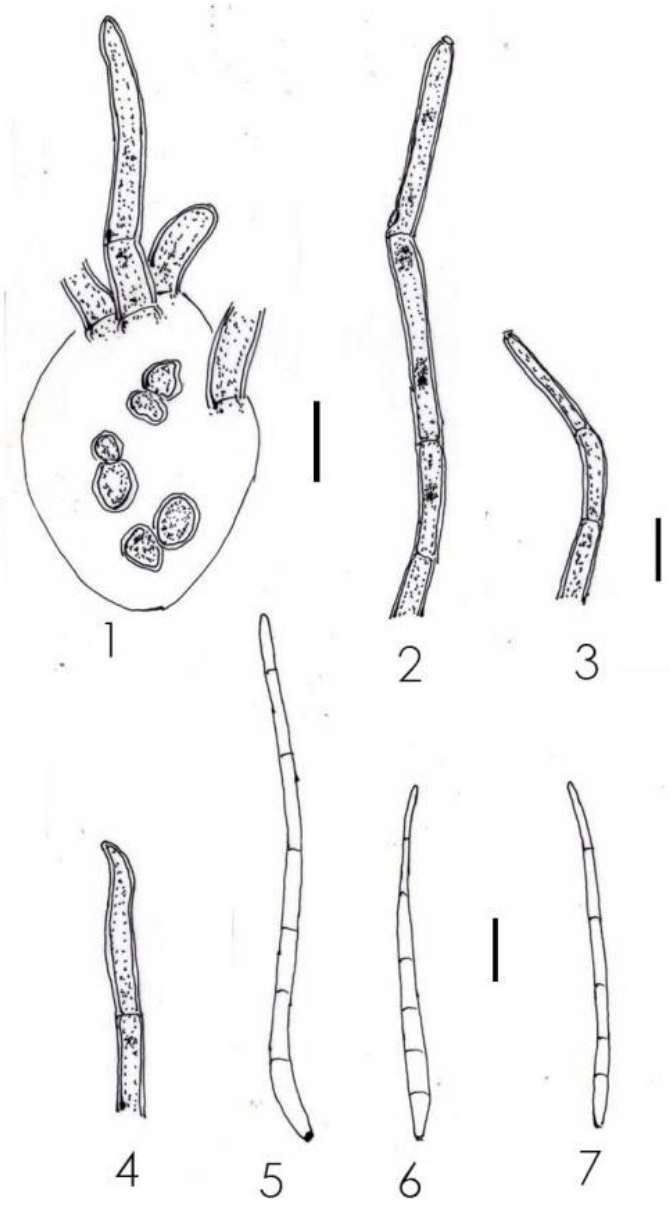

Fig. 16 - Cercospora coffeicola on Coffea arabica from leaf spots: 1. Stroma with attached conidiophores. 2-4. Conidiophore. 57. Conidia. Bars: $1-7=10 \mu \mathrm{m}$.

Cercospora crotalariae Sacc. Syll. Fung. 22: 129, 1913. Figs 18-19.

= Cercospora crotalariae-junceae Sawada, J. Taihoku Soc. Agric. 7: 27, 1942.

Teleomorph: Mycosphaerella crotalariae (Petch) Hans f., Proc. Linn. Soc. London 153: 121, 1942. 


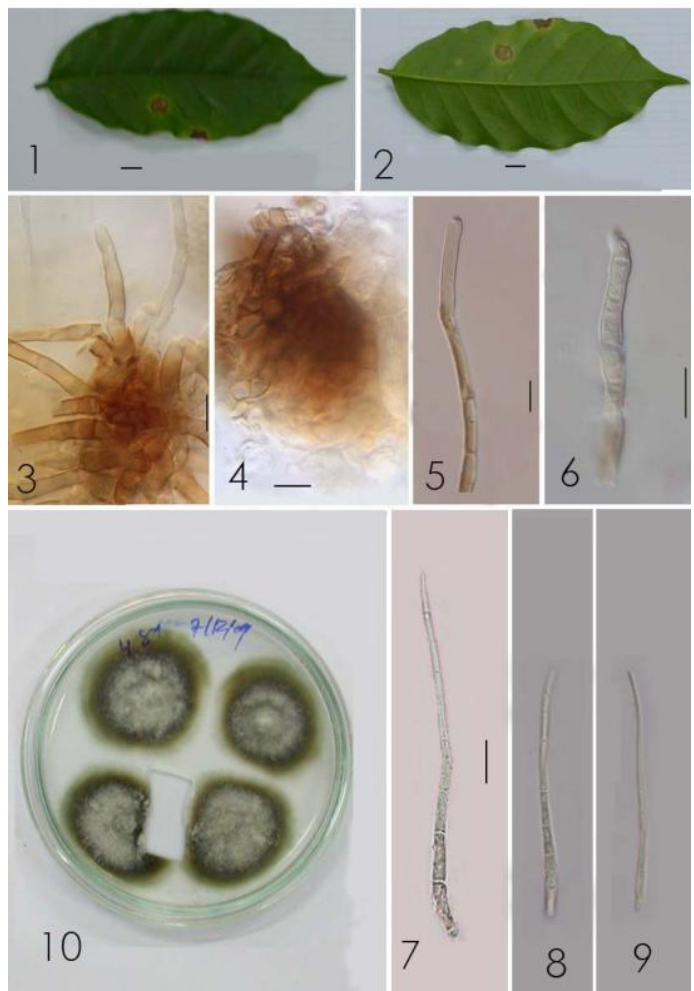

Fig. 17 - Cercospora coffeicola on Coffea arabica from leaf spots: 1-2. Leaf spots on host leaves (1. upper surface, 2. lower surface). 3-4 Stromata with attached conidiophores. 56. Conidiophore. 7-9. Conidia. 10. Culture. Bars: $1-2=10 \mathrm{~mm} .3-9=10 \mu \mathrm{m} .10=10 \mathrm{~mm}$.

$\equiv$ Sphaerella crotalariae Petch, Ann. Roy. Bot. Gard. 3: 2, 1906.

Leaf spots subcircular to irregular, 1-5 $\mathrm{mm}$ diam., grey-brown to dark brown in the centre, dark brown margin. Colonies amphigenous, scattered, dark brown. Mycelium internal; hyphae branched, $2-5 \mu \mathrm{m}$ wide $(\bar{x}=$ $4.33 \mu \mathrm{m}, \mathrm{n}=6$ ), septate, constricted at the septa, distance between septa 7-20 $\mu \mathrm{m}(\bar{x}=$ $13.83 \mu \mathrm{m}, \mathrm{n}=6$ ), brownish or green-hyaline, wall $0.5-1 \mu \mathrm{m}$ wide $(\bar{x}=0.85 \mu \mathrm{m}, \mathrm{n}=6)$, smooth, forming plate-like plectenchymatous stromatic hyphal aggregations. Stromata developed, oval to ellipsoidal, $25-45 \mu \mathrm{m}$ diam. $(\bar{x}=22.5 \mu \mathrm{m}, \mathrm{n}=9$ ), brown, substomatal, intraepidermal, composed of swollen hyphal cells, subglobose, rounded and angular in outline, $5-12 \mu \mathrm{m}$ wide $(\bar{x}=8.6 \mu \mathrm{m}, \mathrm{n}=30)$, brown to dark brown, wall $0.5-1 \mu \mathrm{m}$ wide $(\bar{x}=$ $0.89 \mu \mathrm{m}, \mathrm{n}=30$ ). Conidiophores formed singly or fasciculate, arising from stromata (1-11 per fascicle), emerging through stomata, unbranched, straight to curved, cylindrical, 20$148 \times 5-6 \mu \mathrm{m}(\bar{x}=78.3 \times 5.33 \mu \mathrm{m}, \mathrm{n}=15)$, 1-12-septate, distance between septa 7-20 $\mu \mathrm{m}$ long $(\bar{x}=14.5 \mu \mathrm{m}, \mathrm{n}=30)$, medium brown, paler at the apex, wall $0.5-1 \mu \mathrm{m}$ wide $(\bar{x}=$ $0.76 \mu \mathrm{m}, \mathrm{n}=30$ ), smooth, 0-2-times geniculate, width uniform. Conidiogenous cells integrated, terminal or intercalary, cylindrical, 12-17 × 4-5 $\mu \mathrm{m},(\bar{x}=14 \times 4.67 \mu \mathrm{m}, \mathrm{n}=9)$, pale brown; conidiogenous loci conspicuous, subcircular, $2-4 \mu \mathrm{m}$ wide $(\bar{x}=3 \mu \mathrm{m}, \mathrm{n}=9)$, dark brown, wall $0.5-1 \mu \mathrm{m}$ thick $(\bar{x}=0.6 \mu \mathrm{m}$, $\mathrm{n}=9$ ). Conidia solitary, acicular, straight to curved, $40-320 \times 3-4 \mu \mathrm{m}(\bar{x}=180 \times 3.5 \mu \mathrm{m}$, $\mathrm{n}=7$ ), 3-26-septate, hyaline, thin-walled, 0.3$0.5 \mu \mathrm{m}$ wide $(\bar{x}=0.4 \mu \mathrm{m}, \mathrm{n}=7$ ), smooth, tip acute, base truncate, hila thickened and darkened, $2-3 \mu \mathrm{m}$ wide $(\bar{x}=2.5 \mu \mathrm{m}, \mathrm{n}=7)$, wall of the hila $0.3-0.5 \mu \mathrm{m}$ thick $(\bar{x}=0.35$ $\mu \mathrm{m}, \mathrm{n}=7$ ).

Known hosts - Crotalaria incana L., $C$. juncea L., C. mucronata Desv., C. retusa L., $C$. sericea Burm. f., C. spectabilis Roth., Crotalaria sp., C. striata DC., C. stricta Roxb., Crotalaria uncinella subsp. elliptica (Roxb.) Polhill (new hosts), C. usaramoensis Baker f. (Fabaceae).

Known distribution - Asia: Bangladesh, China, India, Indonesia, Laos, Pakistan, Papua New Guinea, Sri Lanka, Taiwan, Thailand; South America and West Indies: Puerto Rico, Venezuela.

Material examined - Chiang Rai Province, Muang District, Sri Pangsang Village, on the leaves of Crotalaria uncinella subsp. elliptica (Fabaceae), 31 August 2009, P. Phengsintham (P449).

Notes - The collection from Thailand agrees well with the description of $C$. crotaliae published by Hsieh \& Goh (1990) in having a similar size of conidiophores and conidia (conidiophores 45-110 × 4-6 $\mu \mathrm{m}$, conidia 40$110 \times 2.5-4 \mu \mathrm{m})$.

Literature - Saccardo (1913: 129); Chupp (1954: 297); Hsieh \& Goh (1995: 167); Crous \& Braun (2003: 143).

Cercospora diplaziicola A.K. Das., Indian J. Mycol. Res. 27: 37, 1989.

Figs 20-21.

Leaf spots subcircular to irregular, 1-5 $\mathrm{mm}$ diam., grey-brown to dark brown in the centre, dark brown margin. Colonies amphigenous, scattered, dark brown. Mycelium internal; hyphae branched, 2-3 $\mu \mathrm{m}$ wide 


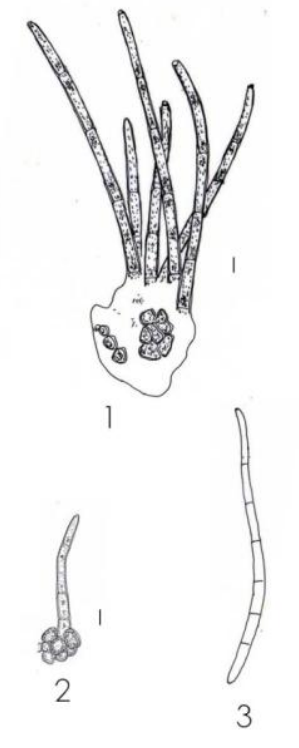

Fig. 18 - Cercospora crotalariae on Crotalaria uncinella subsp. elliptica from leaf spots: 1-2. Stromata with attached conidiophores. 3. Conidium. Bars: $1-3=10$ $\mu \mathrm{m}$.

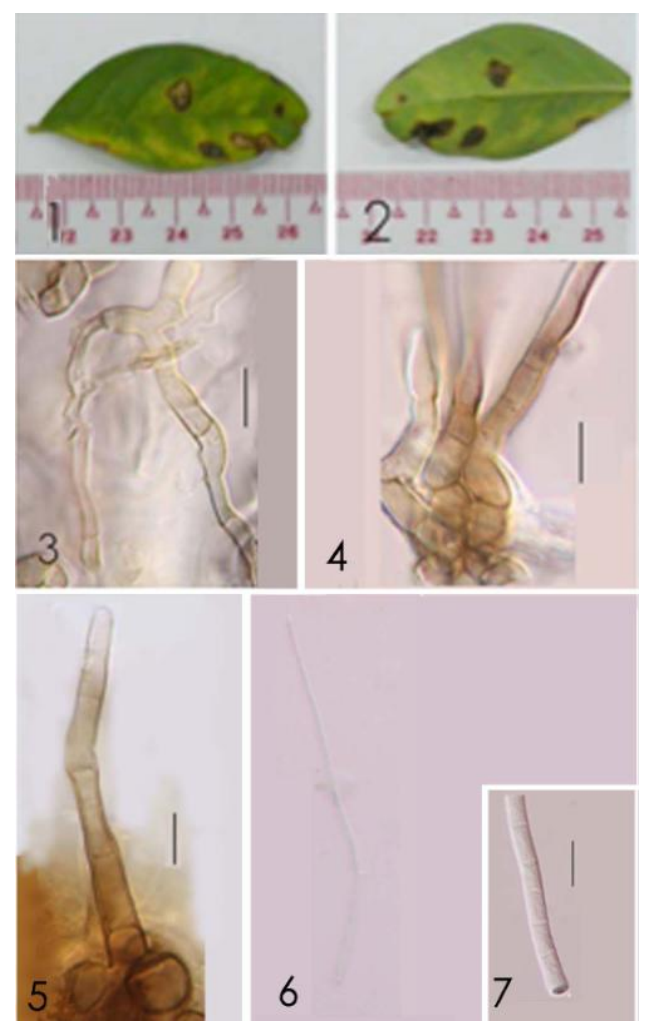

Fig. 19 - Cercospora crotalariae on Crotalaria uncinella subsp. elliptica from leaf spots: 1. Leaf spots on host leaves (1. upper surface, 2. lower surface). 3. Internal hyphae. 4-5. Stromata with attached conidiophores. 6. Conidium. 7. Base of conidium. Bars: $1-2=10$ mm. 3-7 = $10 \mu \mathrm{m}$. $(\bar{x}=2.5 \mu \mathrm{m}, \mathrm{n}=13)$, septate, constricted at the septa, distance between septa 5-10 $\mu \mathrm{m}(\bar{x}=$ $7.5 \mu \mathrm{m}, \mathrm{n}=13$ ), brownish or green-hyaline, wall $0.3-0.5 \mu \mathrm{m}$ wide $(\bar{x}=0.35 \mu \mathrm{m}, \mathrm{n}=13)$, smooth, forming plate-like plectenchymatous stromatic hyphal aggregations. Stromata welldeveloped, oval to ellipsoidal, 15-25 $\mu \mathrm{m}$ diam. $(\bar{x}=20 \mu \mathrm{m}, \mathrm{n}=7$ ), brown, substomatal, intraepidermal, composed of swollen hyphal cells, subglobose, rounded and angular in outline, 6-17 $\mu \mathrm{m}$ wide $(\bar{x}=12 \mu \mathrm{m}, \mathrm{n}=30)$, brown to dark brown, wall $0.5-0.8 \mu \mathrm{m}$ wide $(\bar{x}$ $=0.7 \mu \mathrm{m}, \mathrm{n}=30)$. Conidiophores formed singly or fasciculate, arising from stromata (17 per fascicle), emerging through stomata, unbranched, straight to curved, cylindrical, 40 $76 \times 4-6) \mu \mathrm{m}(\bar{x}=65.1 \times 5 \mu \mathrm{m}, \mathrm{n}=13), 2-4-$ septate, distance between septa $8-28 \mu \mathrm{m}$ long $(\bar{x}=17.8 \mu \mathrm{m}, \mathrm{n}=30)$, medium brown, paler at the apex, wall $0.5-0.8 \mu \mathrm{m}$ wide $(\bar{x}=0.66 \mu \mathrm{m}$, $\mathrm{n}=30$ ), smooth, 0-1-times geniculate, width uniform. Conidiogenous cells integrated, terminal or intercalary, cylindrical, $12-28 \times 3-$ $5 \mu \mathrm{m},(\bar{x}=20.5 \times 3.83 \mu \mathrm{m}, \mathrm{n}=9)$, pale brown; conidiogenous loci conspicuous, subcircular, 2-3 $\mu \mathrm{m}$ wide $(\bar{x}=0.8 \mu \mathrm{m}, \mathrm{n}=9)$, dark brown, wall $0.5-0.8 \mu \mathrm{m}$ thick $(\bar{x}=0.57 \mu \mathrm{m}, \mathrm{n}=9)$. Conidia solitary, acicular, straight to curved, $52-156 \times 3-4 \mu \mathrm{m}(\bar{x}=106.33 \times 3.5 \mu \mathrm{m}, \mathrm{n}=$ 8), 3-18-septate, hyaline, thin-walled $0.3-0.5$ $\mu \mathrm{m}$ wide $(\bar{x}=0.33 \mu \mathrm{m}, \mathrm{n}=8)$, smooth, tip acute, base truncate, hila thicked and darkened, 2-3 $\mu \mathrm{m}$ wide $(\bar{x}=2.4 \mu \mathrm{m}, \mathrm{n}=8)$, wall of the hila $0.3-0.5 \mu \mathrm{m}$ thick $(\bar{x}=0.34 \mu \mathrm{m}, \mathrm{n}=9)$.

Known hosts - Diplazium esculentum (Retz.) Sw. (Woodsiaceae). Thailand.

Known distribution - Asia: India, Laos,

Material examined - Chiang Rai Province, Muang District, Sri Pangsang Village, on the leaves of Diplazium esculentum (Woodsiaceae), 3 August 2009, P. Phengsintham (P450).

Notes - This species is distinct from $C$. apii s. lat. in having acicular to obclavate, 3-6 $\mu \mathrm{m}$ wide conidia, with an obconically truncate base (Crous \& Braun 2003).

Literature - Crous \& Braun (2003).

Cercospora gossypina Cooke, Grevillea 12: 31, 1883.

Figs 22-23.

Teleomorph: Mycosphaerella gossypina 


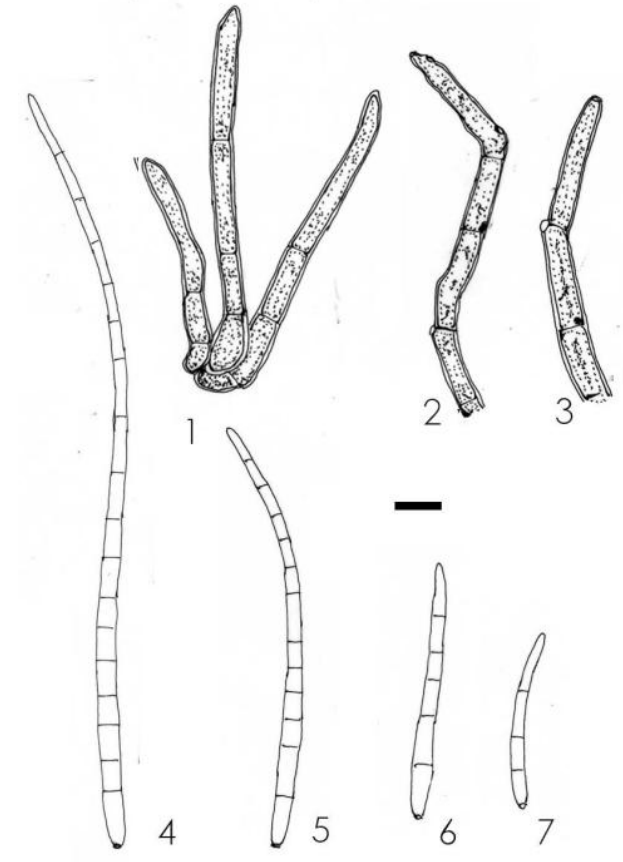

Fig. 20 - Cercospora diplaziicola on Diplazium esculentum from leaf spots: 1. Stroma with attached conidiophores.2-3. Conidiophores. 4-5. Conidia. Bars: $1-7=10$ $\mu \mathrm{m}$.

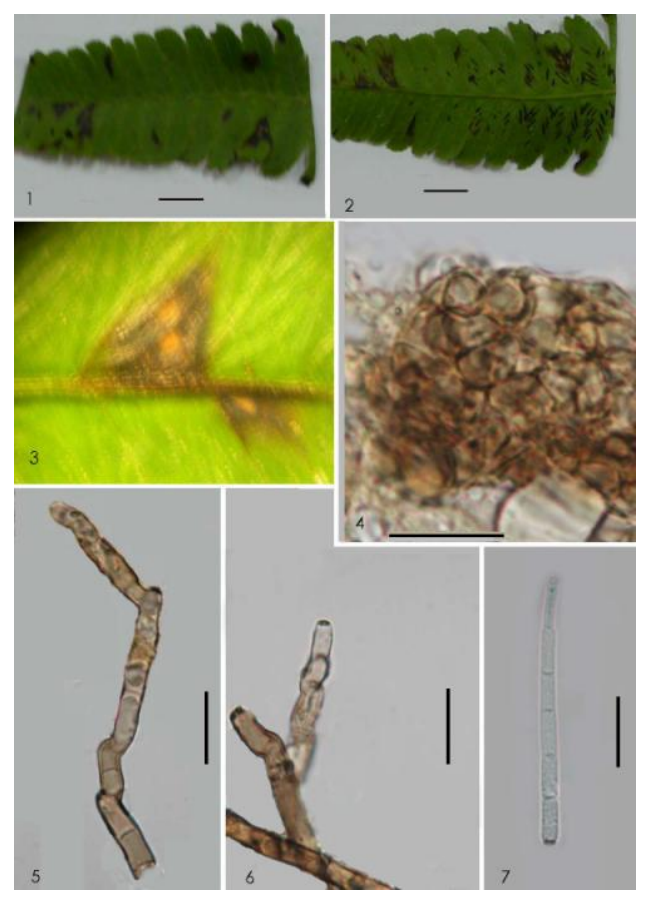

Fig. 21 - Cercospora diplaziicola on Diplazium esculentum from leaf spots: 1-3. Leaf spots on host leaves (1. upper surface, 2. lower surface. 3. leaf spots). 4. Stroma. 5. Conidiophore. 6. Apex of conidiophores. 7. Conidium. Bars: $1-2=10 \mathrm{~mm}$. 3. Not to scale. $4-7=10 \mu \mathrm{m}$.
(G.F. Atk.) Earle, Bull. Alab. Exp. Sta. 107: 309, 1900.

三Sphaerella gossypina G.F. Atk, Bull. Torrey Bot. Club 18: 300, 1891.

Leaf spots small to fairly large, suborbicular to irregular, $1-5 \mathrm{~mm}$ in diam., greybrown in the centre, and with dark brown margin. Colonies hypophyllous, scattered, dark brown. Mycelium internal, inconspicuous. Stromata developed, small to medium-sized, globular to subglobular, substomatal and intraepidermal, $13-50 \mu \mathrm{m}$ in diam. ( $\bar{x}=27$ $\mu \mathrm{m}, \mathrm{n}=9$ ), dark brown to black in mass, composed of swollen hyphal cells, subglobose, rounded to angular in outline, 5-12 $\mu \mathrm{m}$ wide $(\bar{x}=8.3 \mu \mathrm{m}, \mathrm{n}=16)$, brown to dark brown, wall $0.5-1 \mu \mathrm{m}$ wide $(\bar{x}=0.72 \mu \mathrm{m}, \mathrm{n}=16)$, smooth. Conidiophores fasciculate, arising from stromata (3-20 per fascicle), emerging through stomata, unbranched, straight to curved, cylindrical, 50-95 $\times 3.5-6 \mu \mathrm{m}(\bar{x}=$ $74.6 \times 4.81 \mu \mathrm{m}, \mathrm{n}=8), 1-6$-septate, distance between septa $10-35 \mu \mathrm{m}(\bar{x}=19.1 \mu \mathrm{m}, \mathrm{n}=$ 20), medium brown, paler at the apex, wall $0.5-0.8 \mu \mathrm{m}$ wide $(\bar{x}=0.73 \mu \mathrm{m}, \mathrm{n}=20)$, smooth, 0-2-times geniculate. Conidiogenous cells integrated, terminal, cylindrical, $10-35 \times$ 4-6 $\mu \mathrm{m}(\bar{x}=20.5 \times 4.83 \mu \mathrm{m}, \mathrm{n}=6)$, pale brown; conidiogenous loci conspicuous, subcircular, 2-2.5 $\mu \mathrm{m}$ wide $(\bar{x}=2.1 \mu \mathrm{m}, \mathrm{n}=5)$, wall $0.5-0.8 \mu \mathrm{m}$ thick $(\bar{x}=0.68 \mu \mathrm{m}, \mathrm{n}=5)$, thickened and darkened. Conidia solitary, acicular to obclavate, straight to curved, 32-75 $\times 2-4 \mu \mathrm{m}(\bar{x}=56.5 \times 3.5 \mu \mathrm{m}, \mathrm{n}=6), 1-7-$ septate, hyaline to subhyaline, thin-walled 0.3$0.5 \mu \mathrm{m}(\bar{x}=0.33 \mu \mathrm{m}, \mathrm{n}=6)$, smooth; tip acute; base truncate to somewhat obconically truncate, hila thickened and darkened 1-3 $\mu \mathrm{m}$ wide $(\bar{x}=2.21 \mu \mathrm{m}, \mathrm{n}=6)$, wall of the hila $0.3-0.5 \mu \mathrm{m}(\bar{x}=0.35 \mu \mathrm{m}, \mathrm{n}=6)$ thick.

Colonies on PDA after 3 weeks at $25^{\circ} \mathrm{C}$ with pinkish grey mycelium, reaching 8-14 mm diam., hyphae $2-6 \mu \mathrm{m}$ wide $(\bar{x}=4.14 \mu \mathrm{m}$, $\mathrm{n}=14$ ), septate, constricted at the septa, distance between septa $13-40 \mu \mathrm{m}(\bar{x}=22.71$ $\mu \mathrm{m}, \mathrm{n}=14)$, reddish or brownish, wall $0.5-1$ $\mu \mathrm{m}$ wide $(\bar{x}=0.64 \mu \mathrm{m}, \mathrm{n}=14)$, smooth. Conidia not formed in culture.

Known hosts - Gossypium arboreum L. (= G. indicum Medik.), G. barbadense L., G. herbaceum L., G. hirsutum L., G. sturtianum Willis (Malvaceae). 
Known distribution - Africa: Congo, Egypt, Malawi, Mozambique, Nigeria, Sierra Leone, Somalia, South Africa, Sudan, Tanzania, Togo, Uganda, Zambia, Zimbabwe; Asia: Bangladesh, Cambodia, China, India, Japan, Korea, Laos, Myanmar, Pakistan, Papua New Guinea, Sri Lanka, Taiwan, Thailand; Europe: Azerbaijan; North America and West Indies: Cuba, Dominican Rep., Haiti, Jamaica, Mexico, Puerto Rico, Trinidad and Tobago, USA (FL, MS, OK), Virgin Islands; Australia; South America: Argentina, Brazil, Colombia, Peru, Venezuela.

Material examined - Chiang Rai Province, Muang District, Sri Pangsang Village, on leaves of Gossypium herbaceum (Malvaceae), 15 January 2010, P. Phengsintham (P519). GenBank accession no (LSU, KC677919).

Notes - The collection from Thailand agrees well with $C$. gossypina as circumscribed by Chupp (1954), Ellis (1976) and other authors. C. gossypina is part of the $C$. apii complex from which it is morphologically barely distinguishable (Crous \& Braun 2003).

Literature - Chupp (1954: 371); Ellis (1976: 274); Crous \& Braun (2003: 202).

Cercospora malloti Ellis \& Everh., J. Mycol. 4: 114, 1888.

Figs 24-25.

Leaf spots suborbicular to irregular, 1-5 $\mathrm{mm}$ in diam., brown in the centre, and with dark brown margin. Colonies hypophyllous, scattered, dark brown. Mycelium internal, inconspicuous. Stromata developed, small to medium-sized, globular to subglobular, substomatal and intraepidermal, 15-30 $\mu \mathrm{m}$ in diam. $(\bar{x}=25 \mu \mathrm{m}, \mathrm{n}=5)$, dark brown to black in mass, composed of swollen hyphal cells, subglobose, rounded to angular in outline, 5-11 $\mu \mathrm{m}$ wide $(\bar{x}=7.8 \mu \mathrm{m}, \mathrm{n}=8)$, brown to dark brown in mass, composed of swollen hyphal cells, subglobose, rounded to angular in outline, 5-11 $\mu \mathrm{m}$ wide $(\bar{x}=7.8 \mu \mathrm{m}, \mathrm{n}=8)$, brown to dark brown, wall $0.5-0.8 \mu \mathrm{m}$ wide $(\bar{x}$ $=0.61 \mu \mathrm{m}, \mathrm{n}=8$ ), smooth. Conidiophores fasciculate, arising from stromata (1-10 per fascicle), emerging through stomata, not branched, straight to curved, cylindrical, about $32-140 \times 5-6 \mu \mathrm{m}(\bar{x}=83.6 \times 5.6 \mu \mathrm{m}, \mathrm{n}=8)$,
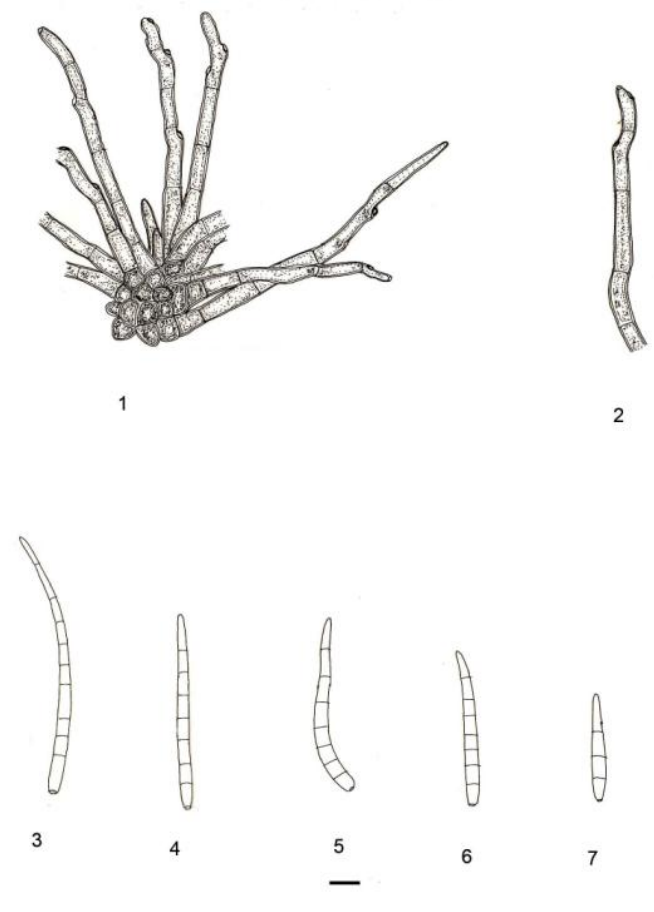

Fig. 22 - Cercospora gossypina on Gossypium herbaceum from leaf spots: 1. Stroma with attached conidiophores. 2. Conidiophore. 3-7. Conidia. Bars: 1-7 = 10 $\mu \mathrm{m}$.

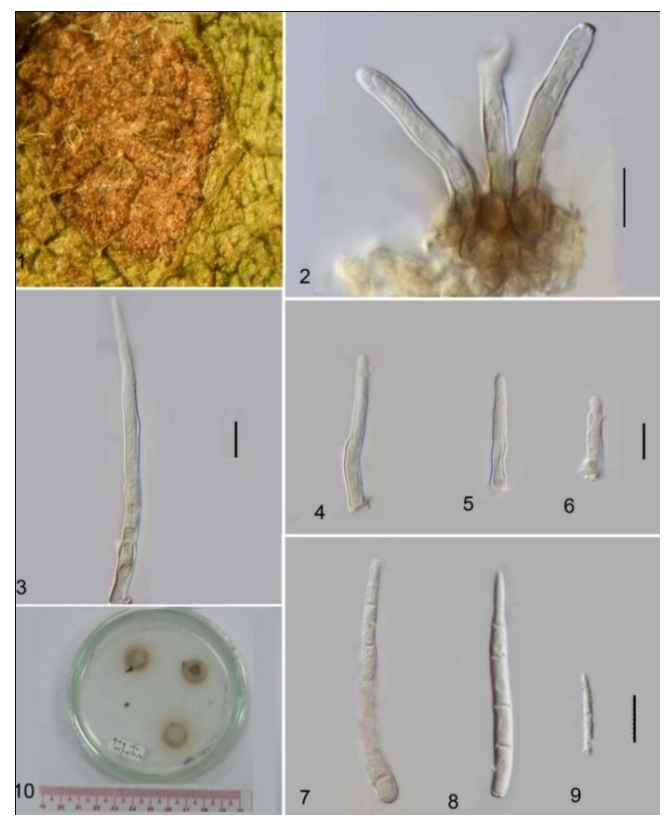

Fig. 23 - Cercospora gossypina on Gossypium herbaceum from leaf spots: 1. Colonies. 2. Stroma with attached conidiophores. 3-6. Conidiophores. 7-9. Conidia. 10. Culture. Bars: 1. Not to scale. $2-9=10 \mu \mathrm{m} .10=10$ $\mathrm{mm}$. 
1-5-septate, distance between septa 12-31 $\mu \mathrm{m}$ $(\bar{x}=21.3 \mu \mathrm{m}, \mathrm{n}=20)$, medium brown, paler at the apex, wall $0.5-0.8 \mu \mathrm{m}$ wide $(\bar{x}=0.65 \mu \mathrm{m}$, $\mathrm{n}=20)$, smooth, 0-1-times geniculate. Conidiogenous cells integrated, terminal, cylindrical, $20-31 \times 4-5 \mu \mathrm{m}(\bar{x}=27 \times 4.67$ $\mu \mathrm{m}, \mathrm{n}=6$ ), pale brown; conidiogenous loci conspicuous, subcircular, $2-3 \mu \mathrm{m}$ wide $(\bar{x}=$ $2.5 \mu \mathrm{m}, \mathrm{n}=5)$, wall $0.5-0.8 \mu \mathrm{m}$ thick $(\bar{x}=$ $0.65 \mu \mathrm{m}, \mathrm{n}=5)$, thickened and darkened. Conidia solitary, acicular to obclavate, straight to curved, $20-146 \times 2-4 \mu \mathrm{m}(\bar{x}=75.8 \times 3.6$ $\mu \mathrm{m}, \mathrm{n}=7), 6-19$-septate, hyaline to subhyaline, thin-walled $0.3-0.5 \mu \mathrm{m}(\bar{x}=0.34 \mu \mathrm{m}$, $\mathrm{n}=6$ ), smooth; tip acute; base truncate to somewhat obconically truncate, hila thickened and darkened $1.5-3 \mu \mathrm{m}$ wide $(\bar{x}=2.5 \mu \mathrm{m}, \mathrm{n}=$ 6), wall of the hila $0.3-0.5 \mu \mathrm{m}(\bar{x}=0.34 \mu \mathrm{m}, \mathrm{n}$ $=6$ ) thick.

Known hosts - Mallotus apelta (Lour.) Müll. Arg., M. japonicus (L. f.) Müll. Arg., M. repandus (Rottler) Müll. Arg. (Euphorbiaceae).

Known distribution - Asia: China, Japan, Thailand; North America: USA (MS).

Material examined - Chiang Rai Province, Muang District, Tadsak water fall, on leaves of Mallotus repandus (Euphorbiaceae), 23 December 2009, P. Phengsintham (P494).

Notes - The collection from Thailand agrees well with $C$. malloti as circumscribed by Chupp (1954). $C$ malloti is part of the $C$. apii complex (Crous \& Braun 2003) from which it is morphologically barely distinguishable.

Literature - Chupp (1954: 224); Hsieh \& Goh (1990: 124); Crous \& Braun (2003: 264).

Cercospora nilghirensis Govindu \& Thirum., Sydowia 9: 224, 1955.

Figs 26-27.

Leaf spots small to medium, suborbicular to irregular, $2-8 \mathrm{~mm}$ in diam., reddish brown in the centre, and with brown margin. Colonies amphigenous, scattered, dark brown. Mycelium internal, inconspicuous. Stromata developed, small to medium-sized, globular to sub-globular, substomatal and intraepidermal, $10-40 \mu \mathrm{m}$ in diam. $(\bar{x}=20.7$ $\mu \mathrm{m}, \mathrm{n}=7$ ), dark brown to black in mass, composed of swollen hyphal cells, subglobose, rounded to angular in outline, $4-8 \mu \mathrm{m}$ wide $(\bar{x}$ $=6.6 \mu \mathrm{m}, \mathrm{n}=15)$, brown to dark brown, wall $0.5-1 \mu \mathrm{m}$ wide $(\bar{x}=0.68 \mu \mathrm{m}, \mathrm{n}=15)$, smooth.

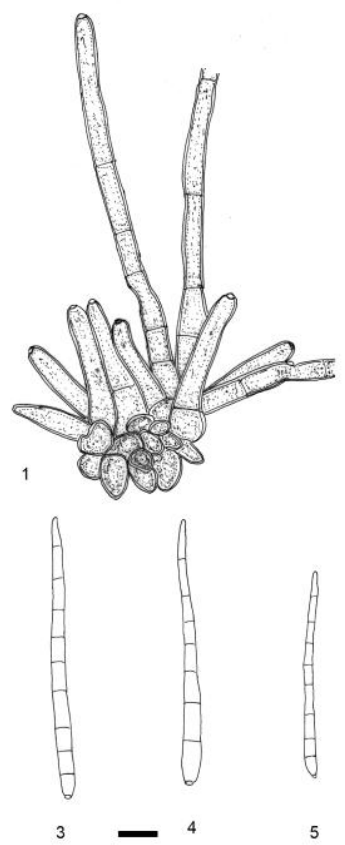

Fig. 24 - Cercospora malloti on Mallotus repandus from leaf spots: 1. Stroma with attached conidiophores. 2-5. Conidia. Bars: 1-5 $=10 \mu \mathrm{m}$.

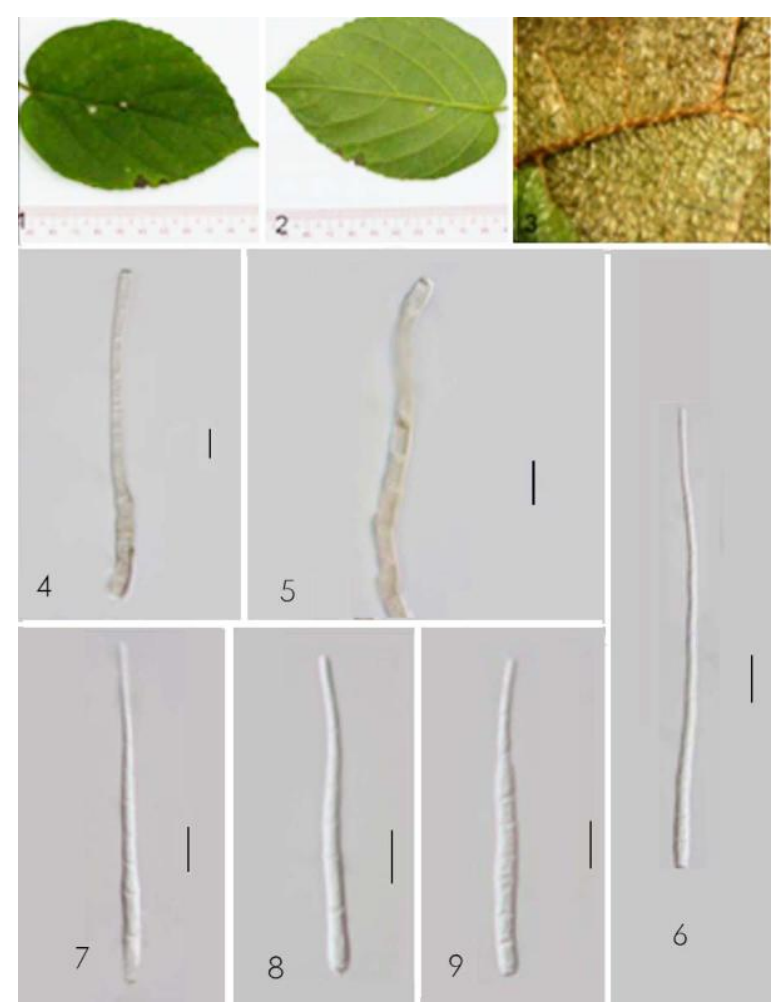

Fig. 25 - Cercospora malloti on Mallotus repandus from leaf spots: 1-2. Leaf spots on host leaves (1. upper surface, 2. lower surface). 3. Colonies. 4-5. Conidiophores. 6-9. Conidia. Bars: $1-2=10 \mathrm{~mm}$. 3. Not to scale. $4-9=10 \mu \mathrm{m}$. 
Conidiophores fasciculate, arising from stromata (1-9 per fascicle), emerging through stomata, not branched, straight to curved, cylindrical, $50-128 \times 3-5 \mu \mathrm{m}(\bar{x}=98.3 \times 3.63$ $\mu \mathrm{m}, \mathrm{n}=9), 2-4$-septate, distance between septa 8-45 $\mu \mathrm{m}(\bar{x}=23.9 \mu \mathrm{m}, \mathrm{n}=30)$, medium brown, paler at the apex, wall $0.5-1 \mu \mathrm{m}$ wide ( $\bar{x}=0.67 \mu \mathrm{m}, \mathrm{n}=30$ ), smooth, 0-2-times geniculate. Conidiogenous cells integrated, terminal, cylindrical, $15-45 \times 3-4 \mu \mathrm{m}(\bar{x}=$ $22.3 \times 2.3 \mu \mathrm{m}, \mathrm{n}=9$ ), pale brown; conidiogenous loci conspicuous, subcircular, 1.5-3.5 $\mu \mathrm{m}$ wide $(\bar{x}=2.84 \mu \mathrm{m}, \mathrm{n}=9)$, wall $0.5-1 \mu \mathrm{m}$ thick ( $\bar{x}=0.77 \mu \mathrm{m}, \mathrm{n}=9$ ), thickened and darkened. Conidia solitary, acicular to obclavate, straight to curved, $14-128 \times 2-3.5$ $\mu \mathrm{m}(\bar{x}=73.3 \times 2.5 \mu \mathrm{m}, \mathrm{n}=11), 1-15$-septate, hyaline to subhyaline, thin-walled $0.3-0.5 \mu \mathrm{m}$ $(\bar{x}=0.33 \mu \mathrm{m}, \mathrm{n}=11)$, smooth; tip acute; base truncate to somewhat obconically truncate, hila thickened and darkened 1.5-3 $\mu \mathrm{m}$ wide $(\bar{x}=$ $2.96 \mu \mathrm{m}, \mathrm{n}=11$ ), wall of the hila $0.3-0.5 \mu \mathrm{m}$ $(\bar{x}=0.4 \mu \mathrm{m}, \mathrm{n}=11)$ thick.

Known hosts - Conyza ambigua DC., C. bonariensis (L.) Cronquist, C. stricta Willd (Asteraceae).

Known distribution - Asia: India, Laos, Thailand.

Material examined - Chiang Rai Province, Muang District, Sri Pangsang Village, on leaves of Conyza bonariensis (Asteraceae), 15 September 2009, P. Phengsintham (P456).

Notes - The collection from Thailand agrees well with the descriptions of $C$. nilghirensis in Vasudeva (1963) and To-anun et al. (2011).

Literature - Vasudeva (1963: 151); Crous \& Braun (2003: 291); To-anun et al. (2011).

Cercospora passifloricola Chupp, A Monograph of the fungus genus Cercospora: 434, 1954.

Figs 28-29.

Leaf spots small to medium, suborbicular to irregular, 1-3 $\mathrm{mm}$ in diam., dark brown in the centre, and with darker brown margin. Colonies amphigenous, scattered, dark brown. Mycelium internal; hyphae branched, $2-4 \mu \mathrm{m}$ wide $(\bar{x}=3 \mu \mathrm{m}, \mathrm{n}=$ 13), septate, constricted at the septa, distance between septa $5-10 \mu \mathrm{m}(\bar{x}=9 \mu \mathrm{m}, \mathrm{n}=13)$,

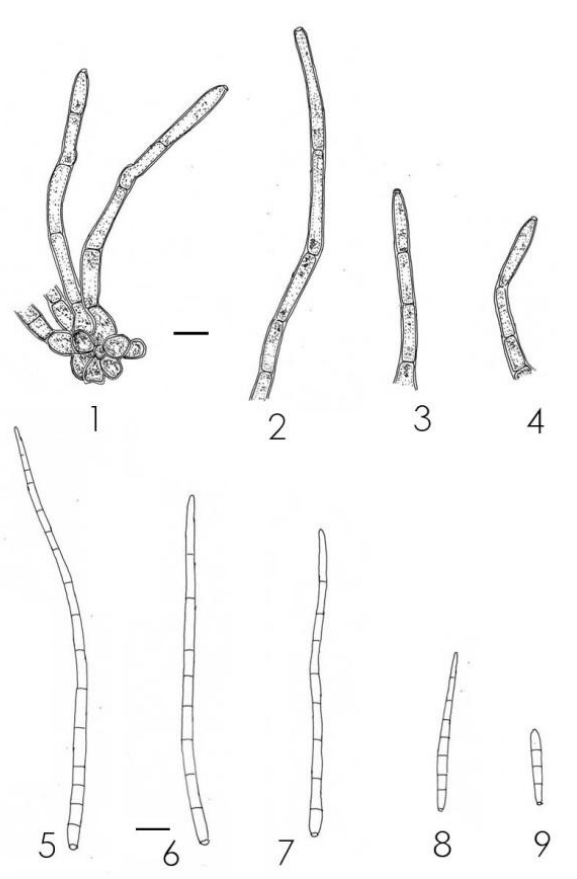

Fig. 26 - Cercospora nilghirensis on Conyza bonariensis from leaf spots: 1 . Stroma with attached conidiophores. 2-4. Conidiophores. 5-9. Conidia. Bars: 1-9=10 $\mu \mathrm{m}$.

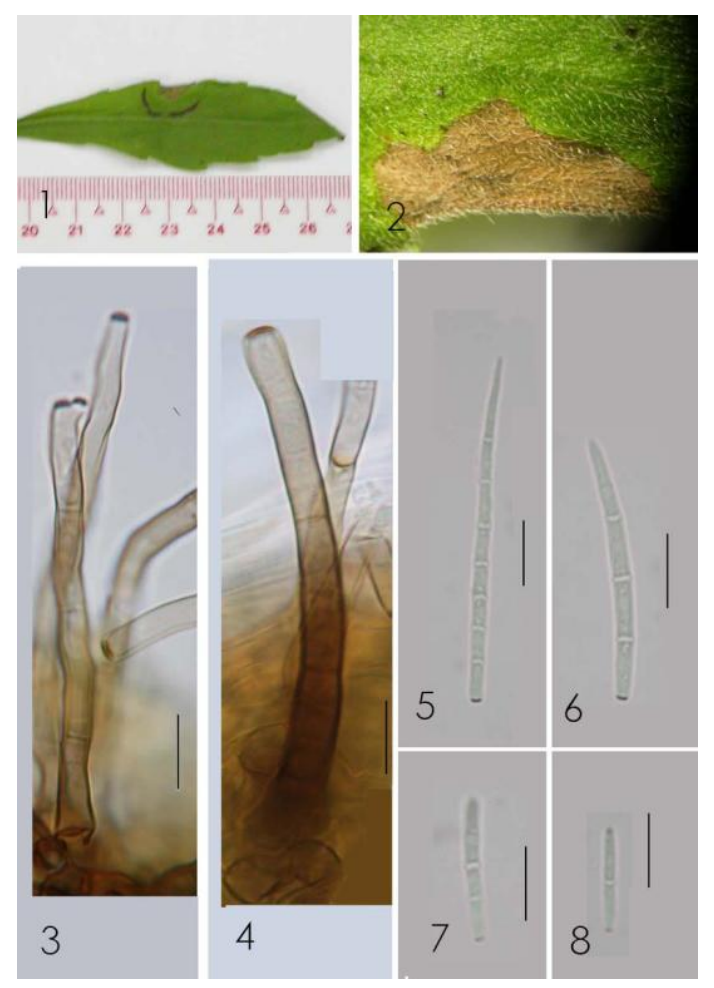

Fig. 27 - Cercospora nilghirensis on Conyza bonariensis from leaf spots: 1. Leaf spots on host leaf (upper surface). 2. Caespituli. 3-4. Stromata with attached conidiophores. 5-8. Conidia. Bars: $1=10 \mathrm{~mm}$. 2 Not to scale. $3-8$ $=10 \mu \mathrm{m}$. 
brownish or green-hyaline, wall $0.3-0.5 \mu \mathrm{m}$ wide ( $\bar{x}=0.46 \mu \mathrm{m}, \mathrm{n}=13)$, smooth, forming plate-like plectenchymatous stromatic hyphal aggregations. Stromata developed, small to medium-sized, globular to subglobular, substomatal and intraepidermal, 10-20 $\mu \mathrm{m}$ in diam. ( $\bar{x}=16.3 \mu \mathrm{m}, \mathrm{n}=8$ ), dark brown to black in mass, composed of swollen hyphal cells, subglobose, rounded to angular in outline, 5-10 $\mu \mathrm{m}$ wide $(\bar{x}=6.6 \mu \mathrm{m}, \mathrm{n}=8)$, brown to dark brown, wall $0.5-0.8 \mu \mathrm{m}$ wide $(\bar{x}$ $=0.62 \mu \mathrm{m}, \mathrm{n}=8$ ), smooth. Conidiophores fasciculate, arising from stromata (1-4 per fascicle), emerging through stomata, unbranched, straight to curved, cylindrical, 32-95 $\times 4-6 \mu \mathrm{m}(\bar{x}=77.6 \times 5.14 \mu \mathrm{m}, \mathrm{n}=7), 1-7-$ septate, distance between septa $12-25 \mu \mathrm{m}(\bar{x}=$ $16.6 \mu \mathrm{m}, \mathrm{n}=30$ ), medium brown, paler at the apex, wall $0.5-0.8 \mu \mathrm{m}$ wide $(\bar{x}=0.7 \mu \mathrm{m}, \mathrm{n}=$ $30)$, smooth, 0-1-times geniculate. Conidiogenous cells integrated, terminal, cylindrical, 14-25 × 4-5 $\mu \mathrm{m}(\bar{x}=19 \times 4.4 \mu \mathrm{m}, \mathrm{n}=7)$, pale brown; conidiogenous loci conspicuous, subcircular, $1.5-3 \mu \mathrm{m}$ wide $(\bar{x}=2.05 \mu \mathrm{m}, \mathrm{n}=7)$, wall $0.5-0.8 \mu \mathrm{m}$ thick $(\bar{x}=0.56 \mu \mathrm{m}, \mathrm{n}=7)$, thickened and darkened. Conidia solitary, acicular to obclavate, straight to curved, 29$102 \times 2-3 \mu \mathrm{m}(\bar{x}=72 \times 2.4 \mu \mathrm{m}, \mathrm{n}=11), 3-9$ septate, hyaline to subhyaline, thin-walled $0.3-$ $0.5 \mu \mathrm{m}(\bar{x}=0.34 \mu \mathrm{m}, \mathrm{n}=11)$, smooth; tip acute; base truncate to somewhat obconically truncate, hila thickened and darkened 1.5-3 $\mu \mathrm{m}$ wide $(\bar{x}=2.2 \mu \mathrm{m}, \mathrm{n}=11)$, wall of the hila $0.3-0.5 \mu \mathrm{m}(\bar{x}=0.34 \mu \mathrm{m}, \mathrm{n}=11)$ thick.

Known hosts - Passiflora coerulea L., P. edulis Sims, P. foetida L., Passiflora sp. (Passifloraceae).

Known distribution - Africa: Zimbabwe; Asia: China, Thailand; North America and West Indies: Barbados; South America: Venezuela.

Material examined - Chiang Rai Province, Muang District, Sri Pangsang Village, on leaves of Passiflora foetida (Passifloraceae), 11 June 2011, P. Phengsintham (P643).

Notes - The collection from Thailand agrees well with $C$. passifloricola as circumscribed by Chupp (1954) (conidiophores 10-55 $\times 2-5 \mu \mathrm{m}$ and conidia $30-120 \times 2-4 \mu \mathrm{m})$.
Literature - Chupp (1954: 434); Crous \& Braun (2003: 309).

Cercospora senecionis-walkeri Phengsintham; Chukeatirote; McKenzie; Hyde \& U. Braun, Pl. Pathol. \& Quarantine 2(1): 70, 2012.

Figs 30-31.

Leaf spots circular to slightly irregular, 2-3 mm diam., at first dark green, later becoming brown to dark brown in the centre, dark brown margin. Colonies amphigenous, conspicuous, scattered, dark brown. Mycelium internal, internal hyphae branched, 3-4 $\mu \mathrm{m}$ wide $(\bar{x}=3.5 \mu \mathrm{m}, \mathrm{n}=5)$, septate, constricted at the septa, distance between septa $12-14 \mu \mathrm{m}$ $(\bar{x}=13 \mu \mathrm{m}, \mathrm{n}=5)$, subhyaline, wall $0.3 \mu \mathrm{m}$ wide $(\bar{x}=0.3 \mu \mathrm{m}, \mathrm{n}=5)$, smooth. Stromata substomatal, intra-epidermal, ellipsoidal, lenticular $10-25 \mu \mathrm{m}$ diam. $(\bar{x}=18.8 \mu \mathrm{m}, \mathrm{n}=5)$, brown, stromatal cells $4-10 \mu \mathrm{m}$ diam. $(\bar{x}=6$ $\mu \mathrm{m}, \mathrm{n}=17)$, wall $0.5-1 \mu \mathrm{m}$ wide $(\bar{x}=0.78$ $\mu \mathrm{m}, \mathrm{n}=17$ ), smooth. Conidiophores single or fasciculate, arising from stromata (1-8 per fascicle), 0-5 geniculate, cylindrical, straight to curved, $67-170 \times 5-6 \mu \mathrm{m}(\bar{x}=114 \times 5.53 \mu \mathrm{m}$, $\mathrm{n}=19), 0-8$-septate, distance between septa 10-32 $\mu \mathrm{m}(\bar{x}=21 \mu \mathrm{m}, \mathrm{n}=30)$, pale brown or olivaceous-brown; wall $0.5-1 \mu \mathrm{m}$ wide $(\bar{x}=$ $0.82 \mu \mathrm{m}, \mathrm{n}=30$ ), smooth. Conidiogenous cells intergrated, terminal, cylindrical, tapering to the apex, $14-30 \times 4-5 \mu \mathrm{m}(\bar{x}=21,8 \times 4.5 \mu \mathrm{m}$, $\mathrm{n}=8$ ); conidiogenous loci (scars) conspicuous, thickened and darkened, $2-4 \mu \mathrm{m}$ wide $(\bar{x}=$ $3.07 \mu \mathrm{m}, \mathrm{n}=30)$, wall of the loci $0.5-1 \mu \mathrm{m}(\bar{x}$ $=0,54 \mu \mathrm{m}, \mathrm{n}=30$ ); thick. Conidia solitary, clavate, cylindrical-clavate, obclavate, straight to curved, $17-82 \times 4-7 \mu \mathrm{m}(\bar{x}=50.66 \times 5.66$ $\mu \mathrm{m}, \mathrm{n}=9$ ), $0-8$-sepate, slightly constricted at the septa, subhyaline or olivaceous-brown, smooth, wall $0.3-0.5 \mu \mathrm{m}$ thick $(\bar{x}=0.37 \mu \mathrm{m}, \mathrm{n}$ =9), apex subobtuse, based truncate, hila 2-3 $\mu \mathrm{m}$ wide $(\bar{x}=2.2 \mu \mathrm{m}, \mathrm{n}=9)$, wall of the hila $0.5 \mu \mathrm{m}$ wide $(\bar{x}=0.5 \mu \mathrm{m}, \mathrm{n}=9)$, darkened.

Known hosts - Senecio walkeri Arn. (Asteraceae).

Thailand.

Known distribution - Asia: Laos,

Material examined - Chiang Rai Province, Muang District, Sri Pangsang Village, on leaves of Senecio walkeri (Asteraceae), 11 August 2009, P. Phengsintham (P434, MFLU 10-00318). 


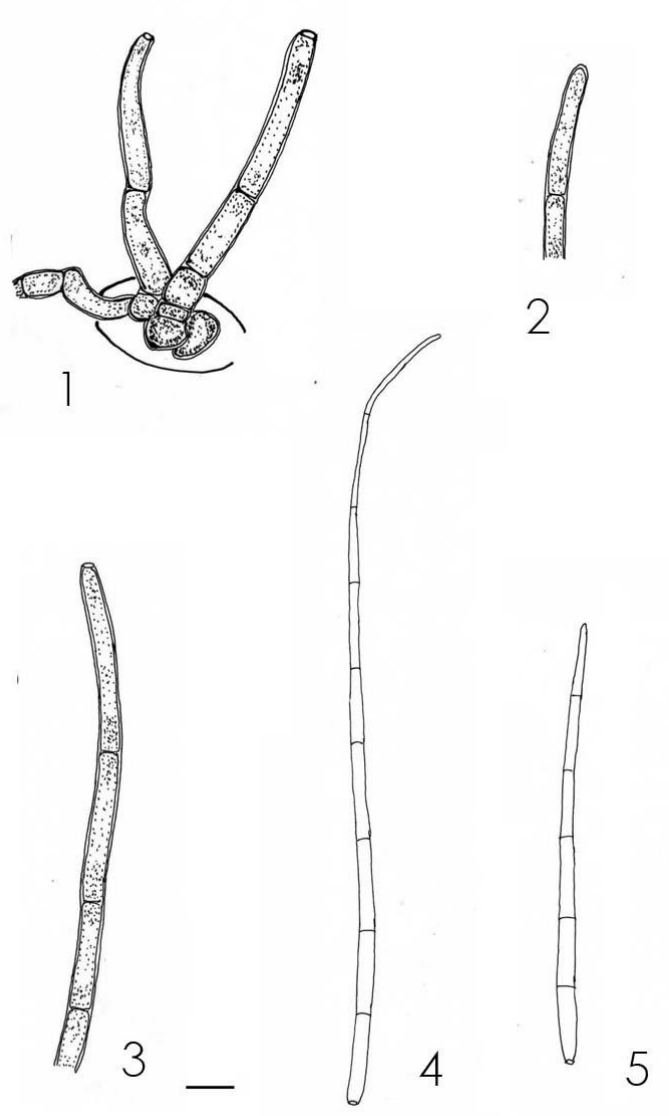

Fig. 28 - Cercospora passifloricola on Passiflora foetida from leaf spots: 1. Stroma with attached conidiophores. 2-3. Conidiophore. 4-5. Conidia. Bars $=10 \mu \mathrm{m}$.

Notes - The collection from Thailand is similar to $C$. senecionis-walkeri from Laos (Phengsintham et al., 2012).

Cercospora sidicola Ellis \& Everh., J. Mycol. 5: 72, 1889.

Figs 32-33.

= Cercospora densissima Speg., Anales Mus. Nac. Hist. Nat. Buenos Aires, Ser. 2(3): 341, 1899.

Leaf spots small to medium, suborbicular to irregular, $1-5 \mathrm{~mm}$ in diam., dark brown in the centre, and with brown to yellowish margin. Colonies amphigenuous, scattered, grey brown. Mycelium internal; hyphae branched, 2-4 $\mu \mathrm{m}$ wide ( $\bar{x}=3 \mu \mathrm{m}, \mathrm{n}=13$ ), septate, constricted at the septa, distance between septa $5-15 \mu \mathrm{m}(\bar{x}=11 \mu \mathrm{m}, \mathrm{n}=13)$, brownish or green-hyaline, wall $0.5-0.8 \mu \mathrm{m}$ wide $(\bar{x}=0.57 \mu \mathrm{m}, \mathrm{n}=13)$, smooth, forming plate-like plectenchymatous stromatic hyphal aggregations. Stromata developed, small to medium-sized, globular to subglobular, substomatal and intra-epidermal, 7-25 $\mu \mathrm{m}$ in diam. $(\bar{x}=16.5 \mu \mathrm{m}, \mathrm{n}=7)$, dark brown to black in mass, composed of swollen hyphal cells, subglobose, rounded to angular in outline, $4-13 \mu \mathrm{m}$ wide $(\bar{x}=7.8 \mu \mathrm{m}, \mathrm{n}=15)$, brown to dark brown, wall $0.5-1 \mu \mathrm{m}$ wide $(\bar{x}$ $=0.88 \mu \mathrm{m}, \mathrm{n}=15)$, smooth. Conidiophores fasciculate, arising from stromata (1-8 per fascicle), emerging through stomata, unbranched, straight to curved, cylindrical, 50-131 × 4-5 $\mu \mathrm{m}(\bar{x}=89 \times 4.67 \mu \mathrm{m}, \mathrm{n}=$ 11), 2-7-septate, distance between septa 8 $32 \mu \mathrm{m}(\bar{x}=17.8 \mu \mathrm{m}, \mathrm{n}=30)$, medium brown, paler at the apex, wall $0.5-0.8 \mu \mathrm{m}$ wide $(\bar{x}=0.64 \mu \mathrm{m}, \mathrm{n}=30)$, smooth, $0-2-$ times geniculate. Conidiogenous cells integrated, terminal, cylindrical, 18-27 × 3-5 $\mu \mathrm{m}(\bar{x}=23 \times 4 \mu \mathrm{m}, \mathrm{n}=8)$, pale brown; conidiogenous loci conspicuous, sub-circular, $2-3 \mu \mathrm{m}$ wide $(\bar{x}=2.75 \mu \mathrm{m}, \mathrm{n}=8)$, wall $0.5-$ $0.8 \mu \mathrm{m}$ thick $(\bar{x}=0.75 \mu \mathrm{m}, \mathrm{n}=8)$, thickened and darkened. Conidia solitary, acicular to obclavate, straight to curved, 44-105 × 2-6 $\mu \mathrm{m}(\bar{x}=64 \times 3.5 \mu \mathrm{m}, \mathrm{n}=10), 4-8$-septate, hyaline to subhyaline, thin-walled $0.3-0.5$ $\mu \mathrm{m}(\bar{x}=0.33 \mu \mathrm{m}, \mathrm{n}=10)$, smooth; tip acute; base truncate to somewhat obconically truncate, hila thickened and darkened 1.5-3 $\mu \mathrm{m}$ wide $(\bar{x}=1.45 \mu \mathrm{m}, \mathrm{n}=15)$, wall of the hila $0.3-0.5 \mu \mathrm{m}(\bar{x}=0.35 \mu \mathrm{m}, \mathrm{n}=15)$ thick.

Known hosts - Sida acuta Burm. f., S. cordifolia (DC.) Fryxell, S. mysorensis Wight \& Arn., S. rhombifolia L., S. spinosa L., Sida sp. (Malvaceae).

Known distribution - Asia: China, India, Thailand; North America and West Indies: Cuba, Dominican Republic, Panama, Puerto Rico, USA (FL, LA, TX), Virgin Islands; South America: Argentina, Brazil.

Material examined - Chiang Rai Province, Wiang Chiang Rung District, Tadsak waterfall, on leaves of Sida mysorensis (Malvaceae), 23 December 2009, P. Phengsintham (MFLU10-0312). GenBank accession no (ITS, KC677888; LSU, KC677922).

Notes - The collection from Tadsak waterfall, Chiang Rai Province agrees with Cercospora sidicola as circumscribed by Chupp (1954), but differs in forming distinct leaf spots. 

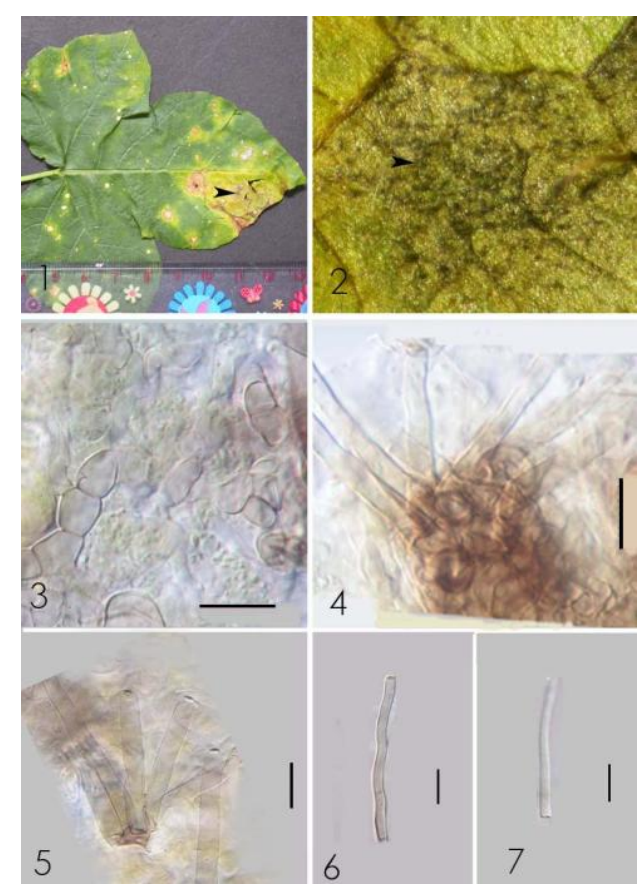

Fig. 29 - Cercospora passifloricola on Passiflora foetida from leaf spots: 1. Leaf spots on host leaf (upper surface). 2. Colonies. 3. Internal hyphae. 4-5. Stromata with attached conidiophores. 6. Conidiophore. 7. Conidia. Bars: $1=10 \mathrm{~mm}$. 2. Not to scale. $3-7=10 \mu \mathrm{m}$.

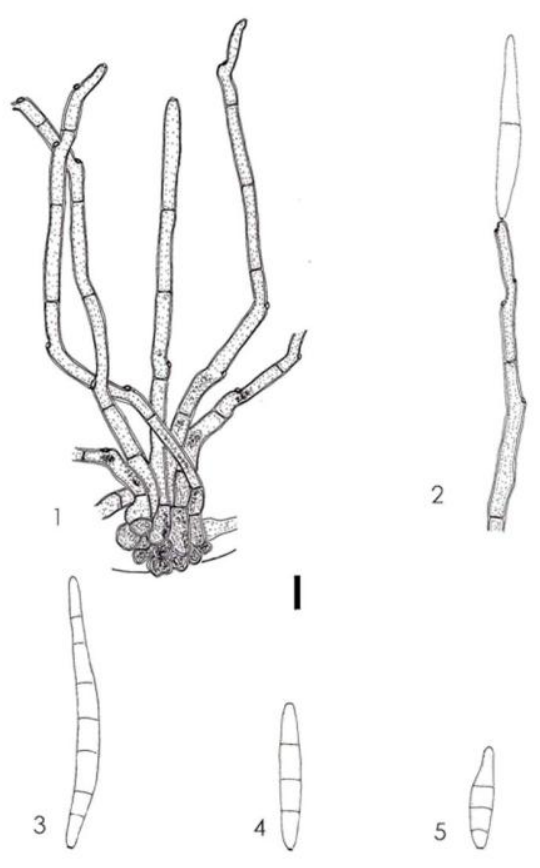

Fig. 30 - Cercospora senecionis-walkeri on Senecio walkeri from leaf spots: 1. Stroma with attached conidiophores. 2. Conidiophore with attached conidium. 3-5. Conidia. Bars: $1-5=10 \mu \mathrm{m}$.

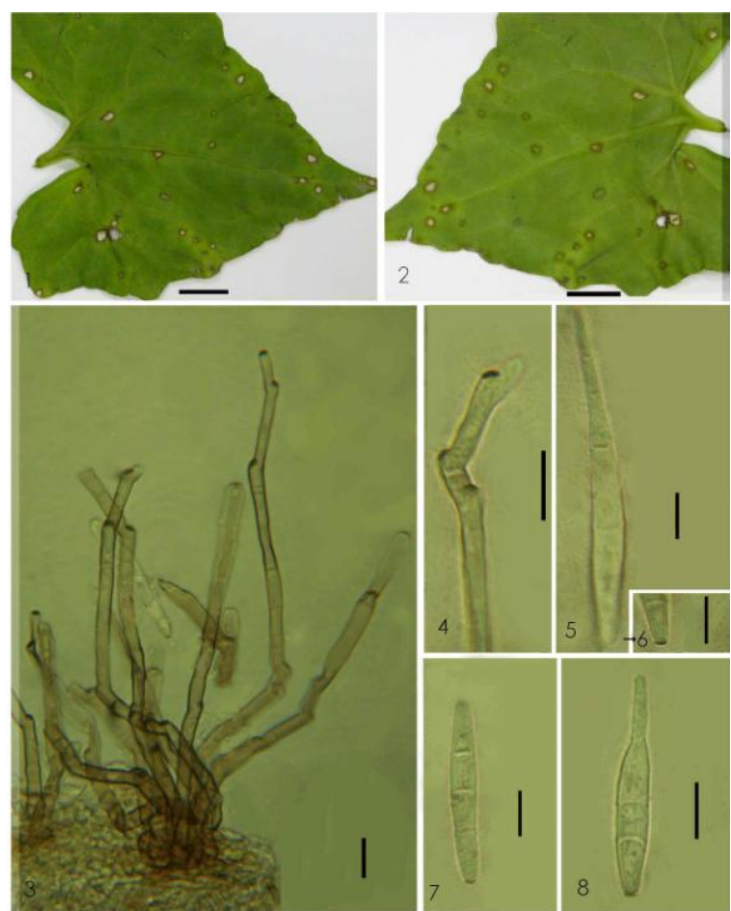

Fig. 31 - Cercospora senecionis-walkeri on Senecio walkeri from leaf spots/lesions: 1-2. Leaf spots on host leaves (1. upper surface, 2. lower surface). 3. Stroma with attached conidiophores. 4. Apex of conidiophore. 5-8. Conidia (6. Base of conidia). Bars: $1-2=10$ $\mathrm{mm}, 3-8=10 \mu \mathrm{m}$.

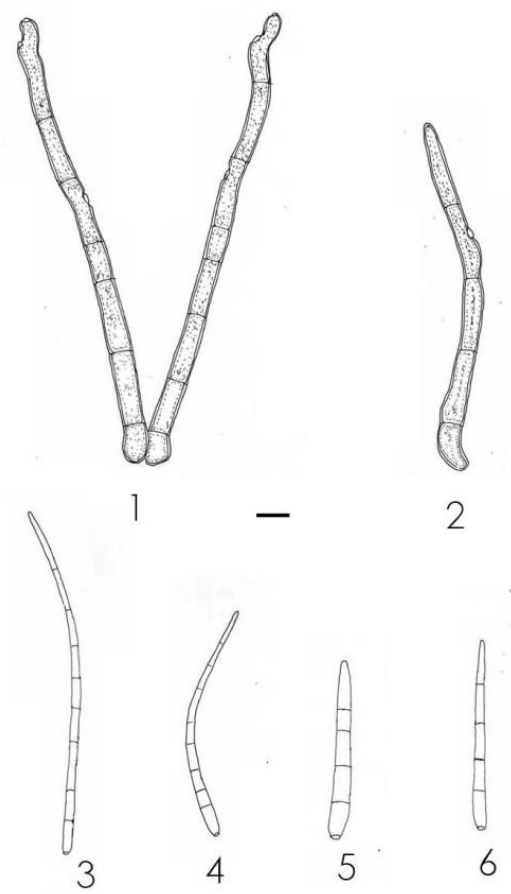

Fig. 32 - Cercospora sidicola on Sida mysorensis from leaf spots: 1. Stromata with attached conidiophores. 2. Conidiophore. 3-6. Conidia. Bars: 1-6=10 $\mu \mathrm{m}$. 


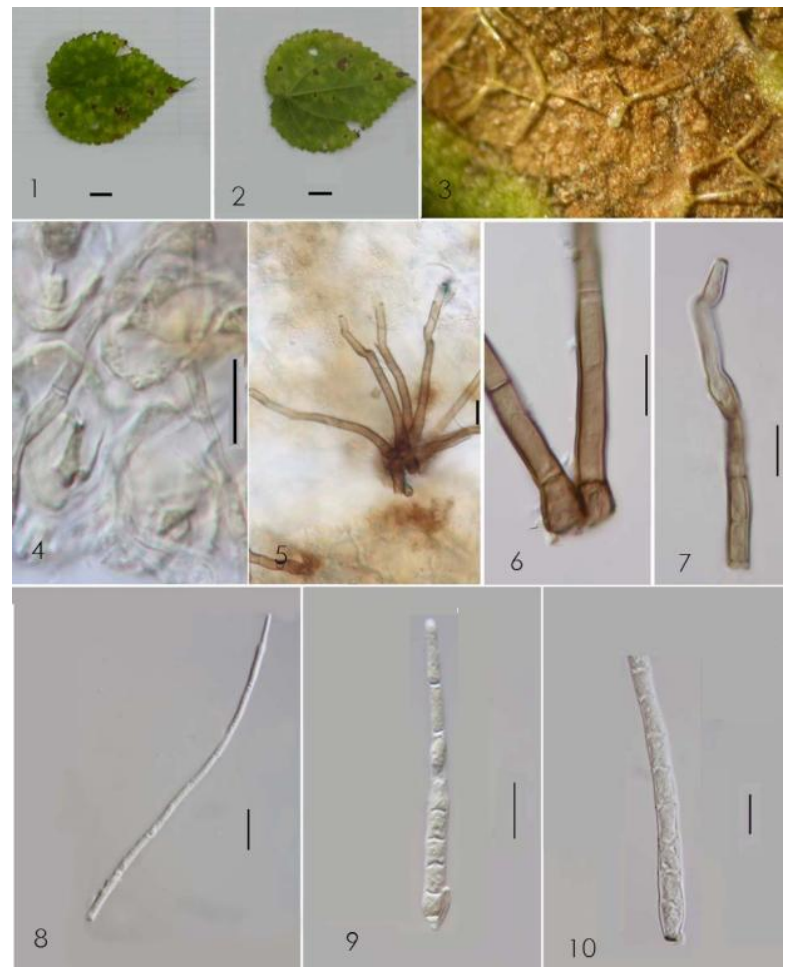

Fig. 33 - Cercospora sidicola on Sida mysorensis from leaf spots: 1-2. Leaf spots on host leaves (1. upper surface, 2. lower surface). 3. Colonies. 4-5. Stromata with attached conidiophores. 6. Conidiophore. 7-8. Conidia. 9. Base of Conidium. Bars: $1-2=10 \mathrm{~mm} .3=$ Not to scale. $4-10=10 \mu \mathrm{m}$.

Literature - Saccardo (1902: 1066); Chupp (1954: 377); Crous \& Braun (2003: 377).

Cercospora sonchi Chupp, A monograph of the fungus genus Cercospora: 154, 1954.

Figs 34-35.

$=$ Cercospora sonchi var. taraxaci Govindu \& Thirum., Sydowia 18: 21, (1964) 1965.

Leaf spots small to fairly large, suborbicular to irregular, 1-15 $\mathrm{mm}$ in diam., reddish brown in the centre, and with dark red-brown margin. Colonies amphigenous, scattered, dark brown. Mycelium internal; hyphae branched, 2-4 $\mu \mathrm{m}$ wide $(\bar{x}=2.6 \mu \mathrm{m}, \mathrm{n}=7)$, septate, constricted at the septa, distance between septa 5-10 $\mu \mathrm{m}$ $(\bar{x}=7 \mu \mathrm{m}, \mathrm{n}=7)$, brownish or green-hyaline, wall approximately $0.3-0.5 \mu \mathrm{m}$ wide $(\bar{x}=0.42$ $\mu \mathrm{m}, \mathrm{n}=7$ ), smooth, forming plate-like plectenchymatous stromatic hyphal aggregations. Stromata developed, small to medium- sized, globular to subglobular, substomatal and intraepidermal, $18-40 \mu \mathrm{m}$ in diam. $(\bar{x}=26$ $\mu \mathrm{m}, \mathrm{n}=5$ ), dark brown to black in mass, composed of swollen hyphal cells, subglobose, rounded to angular in outline, $5-9 \mu \mathrm{m}$ wide $(\bar{x}$ $=7.6 \mu \mathrm{m}, \mathrm{n}=15)$, brown to dark brown, wall $0.5-0.8 \mu \mathrm{m}$ wide $(\bar{x}=0.7 \mu \mathrm{m}, \mathrm{n}=15)$, smooth. Conidiophores fasciculate, arising from stromata (1-7 per fascicle), emerging through stomata, unbranched, straight to curved, cylindrical, $15-190 \times 4-5 \mu \mathrm{m}(\bar{x}=$ $76.24 \times 4.49 \mu \mathrm{m}, \mathrm{n}=15), 1-9$-septate, distance between septa $6-28 \mu \mathrm{m}(\bar{x}=18.27 \mu \mathrm{m}, \mathrm{n}=$ $15)$, medium brown, paler at the apex, wall $0.5-0.8 \mu \mathrm{m}$ wide $(\bar{x}=0.6 \mu \mathrm{m}, \mathrm{n}=15)$, smooth, 0-2-times geniculate. Conidiogenous cells terminal, cylindrical, $13-27 \times 3-4 \mu \mathrm{m}(\bar{x}$ $=22.16 \times 4.21 \mu \mathrm{m}, \mathrm{n}=8)$, pale brown; conidiogenous loci conspicuous, subcircular, $1.5-3 \mu \mathrm{m}$ wide $(\bar{x}=2.35 \mu \mathrm{m}, \mathrm{n}=7)$, wall $0.5-$ $0.8 \mu \mathrm{m}$ thick $(\bar{x}=0.6 \mu \mathrm{m}, \mathrm{n}=7)$, thickened and darkened. Conidia solitary, acicular to obclavate, straight to curved, 78-91 $\times 2-4 \mu \mathrm{m}$ $(\bar{x}=84.58 \times 3.38 \mu \mathrm{m}, \mathrm{n}=5), 7-14$-septate, hyaline to subhyaline, thin-walled $0.3 \mu \mathrm{m}(\bar{x}=$ $0.3 \mu \mathrm{m}, \mathrm{n}=5$ ), smooth, tip acute, base truncate to somewhat obconically truncate; hila thickened and darkened $2-3 \mu \mathrm{m}$ wide $(\bar{x}=$ $2.06 \mu \mathrm{m}, \mathrm{n}=5)$, wall of the hila $0.3-0.5 \mu \mathrm{m}(\bar{x}$ $=0.36 \mu \mathrm{m}, \mathrm{n}=5$ ) thick.

Known hosts - Sonchus arvensis L., $S$. cornutus Hochst. ex Oliv. \& Hiern, $S$. oleraceus L., Sonchus sp., Taraxacum officinale F.H. Wigg., Taraxacum sp. (Asteraceae).

Known distribution - Africa: Sudan; Asia: China, India, Laos, Thailand; North America and West Indies: Barbados, Cuba, USA (VA); Oceania: Vanuatu.

Material examined - Chiang Rai Province, Muang District, Sri Pangsang Village, on leaves of Taraxacum officinale (Asteraceae), 7 July 2010, P. Phengsintham (P600).

Notes - The collection from Thailand agrees well with $C$. sonchi as circumscribed by Chupp (1954) [conidiophores 30-120 × 4-5.5 $\mu \mathrm{m}$, conidia 50-200 $\times 2-3.5 \mu \mathrm{m}]$.

Literature - Chupp (1954: 159); Crous \& Braun (2003: 381). 

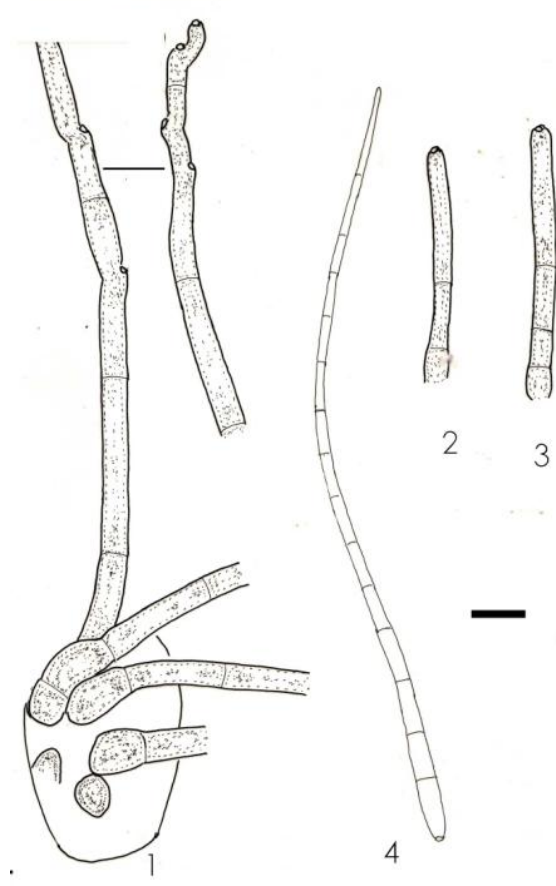

Fig. $34-$ Cercospora sonchi on Taraxacum officinale from leaf spots: 1 . Stroma with attached conidiophores. 2-3. Conidiophores. 4. Conidia. Bars: $1-4=10$ $\mu \mathrm{m}$.

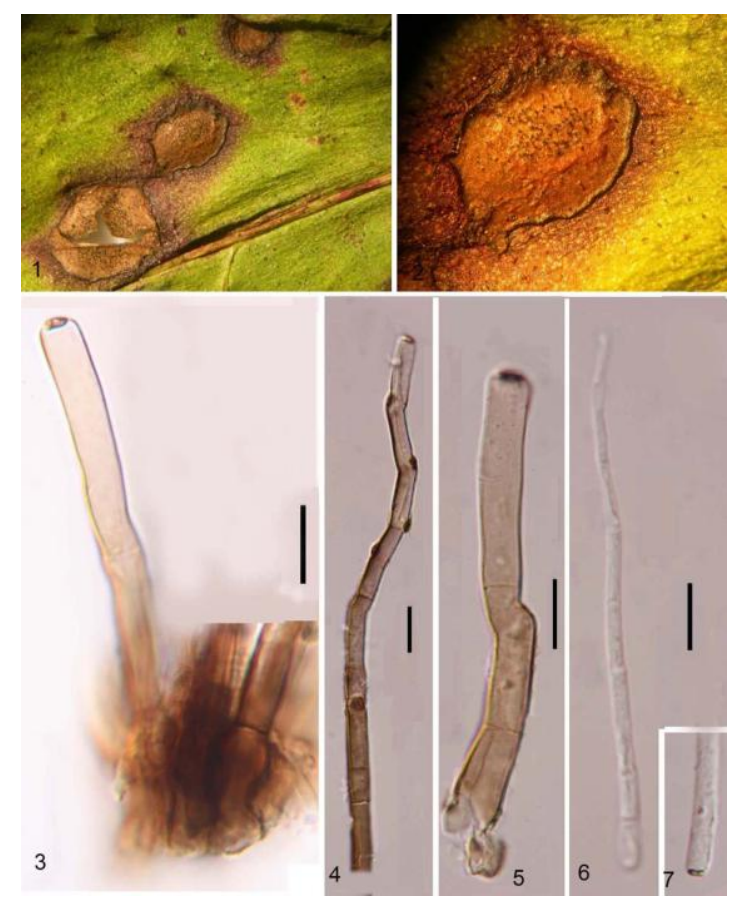

Fig. 35 - Cercospora sonchi on Taraxacum officinale from leaf spots: 1-2. Leaf spots on host leaves and Colonies (lower surface). 3. Stromata with attached conidiophores. 4-5. Conidiophores. 6. Conidium. 7. Based of Conidium. Bars: $1-2=$ Not to scale. $3-7=10$ $\mu \mathrm{m}$.
Cercospora sp. sp. 1.

Figs 36-37.

Leaf spots small to fairly large, suborbicular to irregular, 3-15 $\mathrm{mm}$ in diam., reddish brown in the centre, and reddish brown with yellowish margin. Colonies amphigenous, scattered, dark brown. Mycelium internal, inconspicuous. Stromata developed, small to medium-sized, globular to subglobular, substomatal and intraepidermal, $10-25 \mu \mathrm{m}$ in diam. $(\bar{x}=17.5 \mu \mathrm{m}, \mathrm{n}=5)$, dark brown to black in mass, composed of swollen hyphal cells, subglobose, rounded to angular in outline, 4-8 $\mu \mathrm{m}$ wide $(\bar{x}=6 \mu \mathrm{m}, \mathrm{n}=17)$, brown to dark brown, wall $0.5-0.8 \mu \mathrm{m}$ wide $(\bar{x}$ $=0.6 \mu \mathrm{m}, \mathrm{n}=17)$, smooth. Conidiophores fasciculate, arising from stromata (1-3 per fascicle), emerging through stomata, unbranched, straight to curved, cylindrical, 67$132 \times 4-6 \mu \mathrm{m}(\bar{x}=106 \times 4.75 \mu \mathrm{m}, \mathrm{n}=9), 2$ 5-septate, 0-1-geniculate, distance between septa 10-30 $\mu \mathrm{m}(\bar{x}=18.1 \mu \mathrm{m}, \mathrm{n}=30)$, medium brown, paler at the apex, wall $0.5-0.8$ $\mu \mathrm{m}$ wide $(\bar{x}=0.55 \mu \mathrm{m}, \mathrm{n}=30)$, smooth, $0-2-$ times geniculate. Conidiogenous cells terminal, cylindrical, $22-28 \times 4-5 \mu \mathrm{m}(\bar{x}=25.5 \times 4.25$ $\mu \mathrm{m}, \mathrm{n}=8$ ), pale brown; conidiogenous loci conspicuous, subcircular, $1.5-3 \mu \mathrm{m}$ wide $(\bar{x}=$ $2.12 \mu \mathrm{m}, \mathrm{n}=15)$, wall $0.5-0.8 \mu \mathrm{m}$ thick $(\bar{x}=$ $0.57 \mu \mathrm{m}, \mathrm{n}=15$ ), thickened and darkened. Conidia solitary, acicular to obclavate, straight to curved, $100-202 \times 2-4 \mu \mathrm{m}(\bar{x}=138.67 \times$ $3.33 \mu \mathrm{m}, \mathrm{n}=5)$, 6-16-septate, hyaline to subhyaline, thin-walled $0.25-0.3 \mu \mathrm{m}(\bar{x}=0.28$ $\mu \mathrm{m}, \mathrm{n}=5$ ), smooth, tip acute, base truncate; hila thickened and darkened 1.5-3 $\mu \mathrm{m}$ wide $(\bar{x}$ $=2.16 \mu \mathrm{m}, \mathrm{n}=5$ ), wall of the hila $0.3-0.5 \mu \mathrm{m}$ $(\bar{x}=0.36 \mu \mathrm{m}, \mathrm{n}=5)$ thick.

Known hosts - Crateva religiosa G. Forst. (Capparaceae).

Known distribution - Asia: Thailand.

Material examined - Chiang Rai Province, Muang District, Sri Pangsang Village, on leaves of Crateva religiosa (Capparaceae), 22 August 2010, P. Phengsintham (P618).

Notes - The collection from Thailand is similar to the description of Cercospora apii s. lat. (emend.) published by Crous \& Braun (2003), i.e. the collections on Crateva religiosa can tentatively be assigned to the latter species (complex). Further notes see Cercospora sp. sp. 2. 


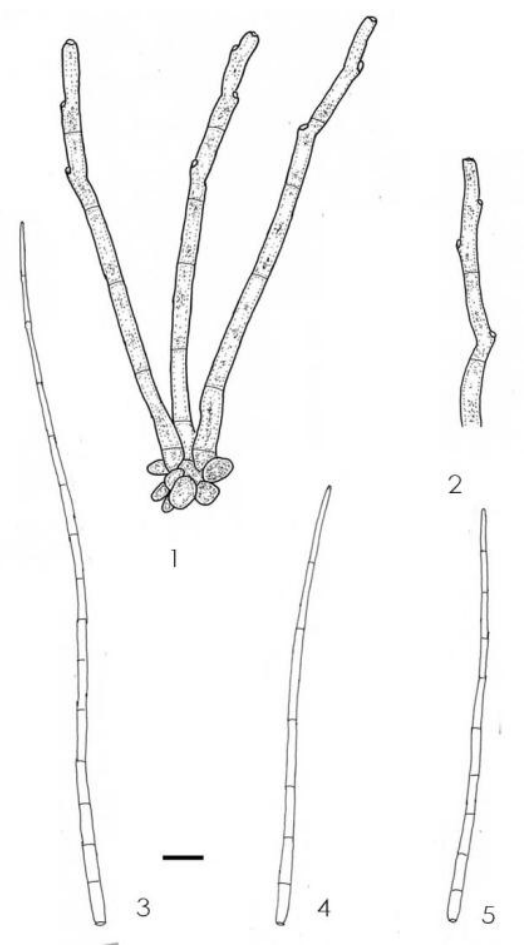

Fig. 36 - Cercospora sp. sp. 1 on Crateva religiosa from leaf spots: 1. Stroma with attached Conidiophores. 2. Conidiophores. 35. Conidia. Bars: $1-5=10 \mu \mathrm{m}$.

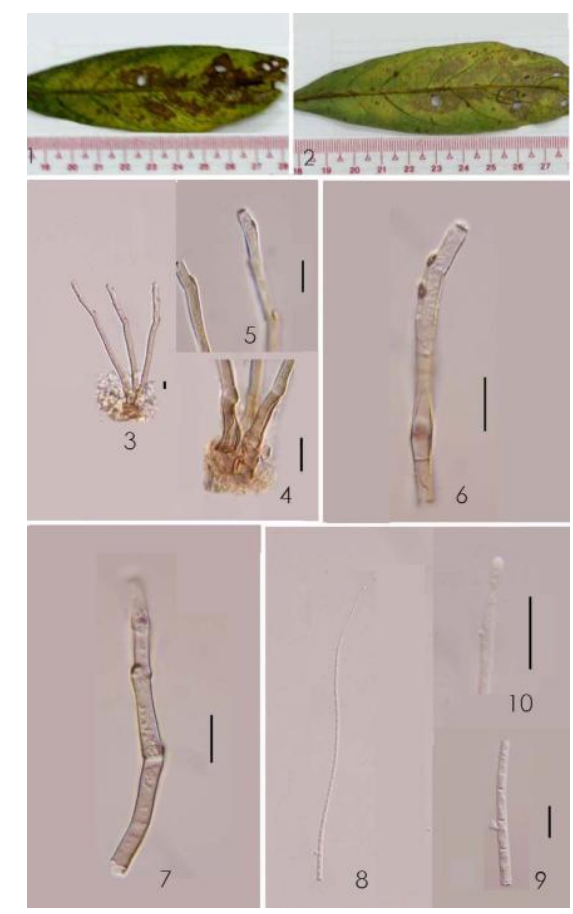

Fig. 37 - Cercospora sp. sp.1 on Crateva religiosa from leaf spots: 1-2. Leaf spots on host leaves and Colonies (lower surface). 3-4. Stromata with attached Conidiophores. 5. Apex of conidiophores. 6-7. Conidiophores. 8. Conidium. 9. Base of Conidium. 10. Apex of conidium. Bars: $1-2=10 \mathrm{~mm} .3-10=10 \mu \mathrm{m}$.
Cercospora sp. sp. 2.

Figs 38-39.

Leaf spots small to medium, suborbicular to irregular, $2-5 \mathrm{~mm}$ in diam., dark brown in the centre, and with brown to yellowish margin. Colonies amphigenuous, scattered, grey-brown. Mycelium internal; hyphae branched, $3-5 \mu \mathrm{m}$ wide $(\bar{x}=3.2 \mu \mathrm{m}, \mathrm{n}$ $=11$ ), septate, constricted at the septa, distance between septa 5-12 $\mu \mathrm{m}(\bar{x}=9.2 \mu \mathrm{m}, \mathrm{n}=11)$, brownish or green-hyaline, wall $0.5-0.8 \mu \mathrm{m}$ wide ( $\bar{x}=0.74 \mu \mathrm{m}, \mathrm{n}=11)$, smooth, forming plate-like plectenchymatous stromatic hyphal aggregations. Stromata developed, small to medium-sized, globular to subglobular, substomatal and intraepidermal, 15-26 $\mu \mathrm{m}$ in diam. ( $\bar{x}=21.5 \mu \mathrm{m}, \mathrm{n}=5)$, dark brown to black in mass, composed of swollen hyphal cells, subglobose, rounded to angular in outline, $6-10 \mu \mathrm{m}$ wide $(\bar{x}=8.3 \mu \mathrm{m}, \mathrm{n}=30)$, brown to dark brown, wall $0.5-0.8 \mu \mathrm{m}$ wide $(\bar{x}$ $=0.65 \mu \mathrm{m}, \mathrm{n}=30$ ), smooth. Conidiophores fasciculate, arising from stromata $(2-13$ per fascicle), emerging through stomata, unbranched, straight to curved, cylindrical, 15-77 $\times 2-4 \mu \mathrm{m}(\bar{x}=62.9 \times 3.61 \mu \mathrm{m}, \mathrm{n}=9), 0-4-$ septate, distance between septa $8-31 \mu \mathrm{m}(\bar{x}=$ $17.6 \mu \mathrm{m}, \mathrm{n}=30)$, medium brown, paler at the apex, wall $0.5-0.8 \mu \mathrm{m}$ wide $(\bar{x}=0.58 \mu \mathrm{m}, \mathrm{n}=$ $30)$, smooth, 0-2-times geniculate. Conidiogenous cells integrated, terminal, cylindrical, 8-31 × 3-4 $\mu \mathrm{m}(\bar{x}=24.4 \times 3.4 \mu \mathrm{m}, \mathrm{n}=5)$, pale brown; conidiogenous loci conspicuous, subcircular, $0.7-3 \mu \mathrm{m}$ wide $(\bar{x}=1.37 \mu \mathrm{m}, \mathrm{n}=$ 7), wall $0.5-0.8 \mu \mathrm{m}$ thick ( $\bar{x}=0.62 \mu \mathrm{m}, \mathrm{n}=7)$, thickened and darkened. Conidia solitary, acicular to obclavate, straight to curved, 54-72 $\times 2-3 \mu \mathrm{m}(\bar{x}=62 \times 2.33 \mu \mathrm{m}, \mathrm{n}=5), 5-9-$ septate, hyaline to subhyaline, thin-walled 0.25-0.3 $\mu \mathrm{m}(\bar{x}=0.28 \mu \mathrm{m}, \mathrm{n}=5)$, smooth; tip acute; base truncate, hila thickened and darkened, $0.7-2 \mu \mathrm{m}$ wide $(\bar{x}=1.17 \mu \mathrm{m}, \mathrm{n}=$ 5), wall of the hila $0.3-0.35 \mu \mathrm{m}(\bar{x}=0.31 \mu \mathrm{m}$, $\mathrm{n}=5$ ) thick.

Colonies on PDA after 3 weeks at $25^{\circ} \mathrm{C}$ are grey-violet to dark brown-violet in the centre, pale brown magin, reaching $10-12 \mathrm{~mm}$ diam.

Known hosts - Celtis timorensis Span. (Cannabaceae).

Known distribution - Asia: Thailand.

Material examined - Chiang Rai Province, Muang District, Sri Pangsang 
Village, on leaves of Celtis timorensis (Cannabaceae), 24 August 2010, P. Phengsintham (P624). GenBank accession no (ITS, KC677889; LSU, KC677923).

Notes - This is the first Cercospora record on a species of Celtis. This collection is morphologically indistinguishable from and belongs to the Cercospora apii complex (Crous $\&$ Braun 2003). Collections on new hosts within this complex should only be referred to as $C$. apii s. lat. (or Cercospora sp. or as belonging to the $C$. apii complex). Without molecular sequence analyses further treatments of collections in this complex are not possible as various plurivorous species, morphologically indistinguishable from $C$. apii, are involved (Groenewald et al., 2012).

Literature - Crous \& Braun (2003); Groenewald et al. (2012).

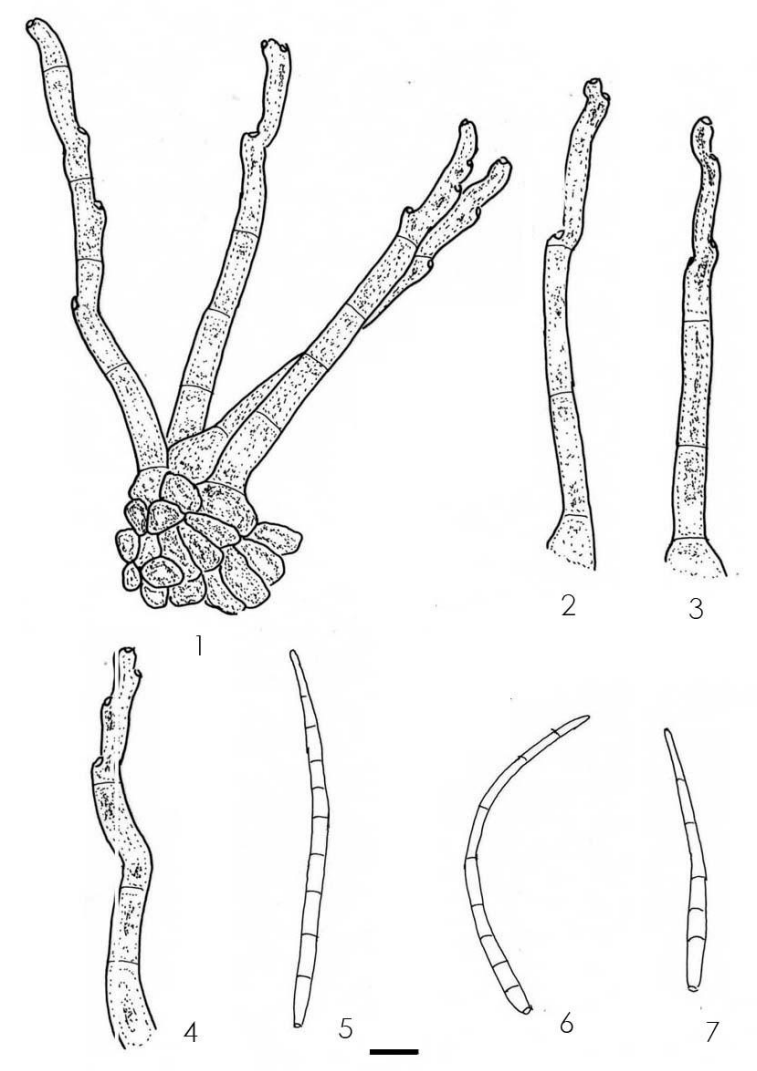

Fig. 38 - Cercospora sp. sp. 2 on Celtis timorensis from leaf spots: 1. Stroma with attached conidiophores. 2-5. Conidiophores. 67. Conidia. Bars: $1-7=10 \mu \mathrm{m}$.

Cercospora sp. sp. 3.

Figs 40-41.

Leaf spots suborbicular to irregular, 1-6 $\mathrm{mm}$ in diam., dark brown in the centre, and with yellowish brown margin. Colonies

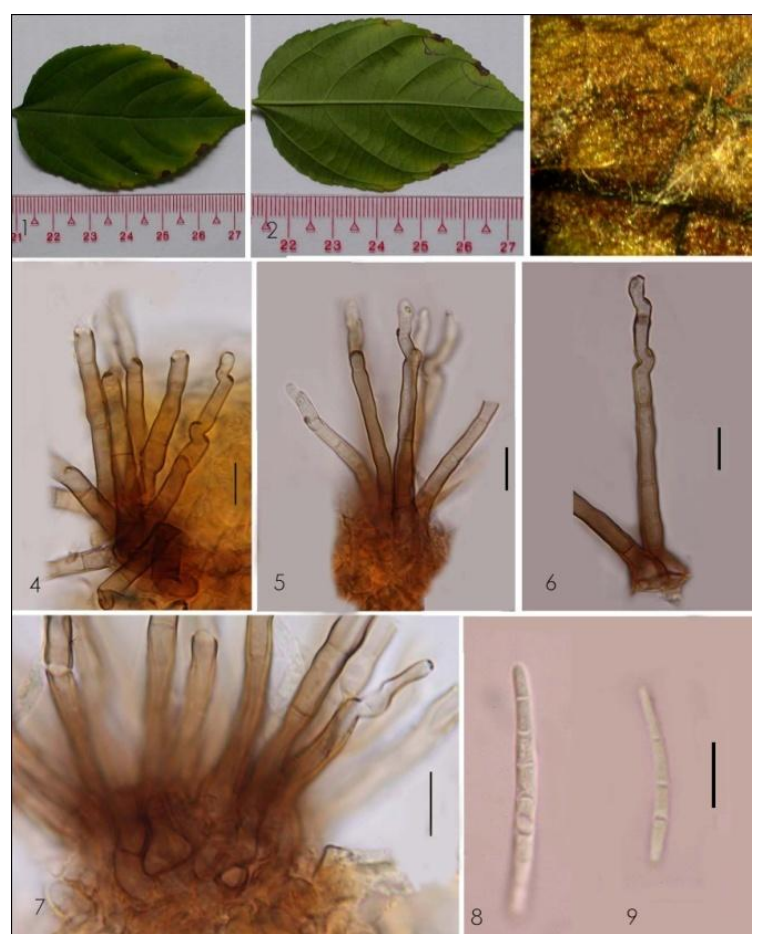

Fig. 39 - Cercospora sp. sp. 2 on Celtis timorensis from leaf spots: 1-2. Leaf spots on host leaves (1. upper surface, 2. lower surface). 3. Colonies. 4-7. Stromata with attached conidiophores. 8-9. Conidia. Bars: $1-2=10$ $\mathrm{mm} .3=$ Not to scale. $4-9=10 \mu \mathrm{m}$.

amphigenous, scattered, dark brown. Mycelium internal, inconspicuous. Stromata developed, small to medium-sized, globular to subglobular, substomatal and intra-epidermal, $3-10 \mu \mathrm{m}$ in diam. $(\bar{x}=5.8 \mu \mathrm{m}, \mathrm{n}=5)$, dark brown to black in mass, composed of swollen hyphal cells, subglobose, rounded to angular in outline, 5-9 $\mu \mathrm{m}$ wide $(\bar{x}=7.6 \mu \mathrm{m}, \mathrm{n}=17)$, brown to dark brown, wall $0.5-0.8 \mu \mathrm{m}$ wide $(\bar{x}$ $=0.62 \mu \mathrm{m}, \mathrm{n}=17$ ), smooth. Conidiophores fasciculate, arising from stromata (1-4 per fascicle), emerging through stomata, unbranched, straight to curved, cylindrical, 40$320 \times 4-7 \mu \mathrm{m}(\bar{x}=147 \times 6 \mu \mathrm{m}, \mathrm{n}=9), 5-7-$ septate, distance between septa 10-28 $\mu \mathrm{m}(\bar{x}=$ $18.3 \mu \mathrm{m}, \mathrm{n}=30)$, medium brown, paler at the apex, wall $0.5-0.8 \mu \mathrm{m}$ wide $(\bar{x}=0.59 \mu \mathrm{m}, \mathrm{n}=$ 30 ), smooth, 0-1-times geniculate. Conidiogenous cells terminal, cylindrical, 15-28 $\times 3-4$ $\mu \mathrm{m}(\bar{x}=21.8 \times 3.25 \mu \mathrm{m}, \mathrm{n}=9)$, pale brown; conidiogenous loci conspicuous, subcircular, $1.5-2.5 \mu \mathrm{m}$ wide $(\bar{x}=2 \mu \mathrm{m}, \mathrm{n}=9)$, wall $0.5-$ $0.8 \mu \mathrm{m}$ thick $(\bar{x}=0.65 \mu \mathrm{m}, \mathrm{n}=9)$, thickened and darkened. Conidia solitary, acicular, 
straight to curved, $163-195 \times 2.5-3 \mu \mathrm{m}(\bar{x}=$ $179 \times 2.75 \mu \mathrm{m}, \mathrm{n}=5), 7-21$-septate, hyaline to subhyaline, thin-walled $0.3-0.5 \mu \mathrm{m}(\bar{x}=0.38$ $\mu \mathrm{m}, \mathrm{n}=5$ ), smooth, tip acute, base truncate; hila thickened and darkened 1.5-2.5 $\mu \mathrm{m}$ wide $(\bar{x}=2 \mu \mathrm{m}, \mathrm{n}=5)$, wall of the hila $0.3-0.5 \mu \mathrm{m}$ $(\bar{x}=0.36 \mu \mathrm{m}, \mathrm{n}=5)$ thick. ceae).

Known host - Ziziphus sp. (Rhamna-

Known distribution - Asia: Thailand.

Material examined - Pha Yao Province, Mae Jai District, Mae Puem National Park, on leaves of Ziziphus sp. (Rhamnaceae), 22 August 2010, P. Phengsintham (MFLU110019).

Notes - The collection MFLU11-0019 from Mae Puem National Park, Pha Yao Province differs to the Cercospora ziziphigena described by $\mathrm{Xu} \&$ Guo (2003) (conidiophores $13.8-92.5 \times 3.5-6.3 \mu \mathrm{m}$ and conidia 17.5-76.8 $\times 3.1-5.3 \mu \mathrm{m})$ in having much longer, narrower conidia. A true Cercospora s. str. quite close to Cercospora apii s. lat. (Crous \& Braun, 2003).

Literature - Crous \& Braun (2003: 435); Xu \& Guo (2003: 6-8).

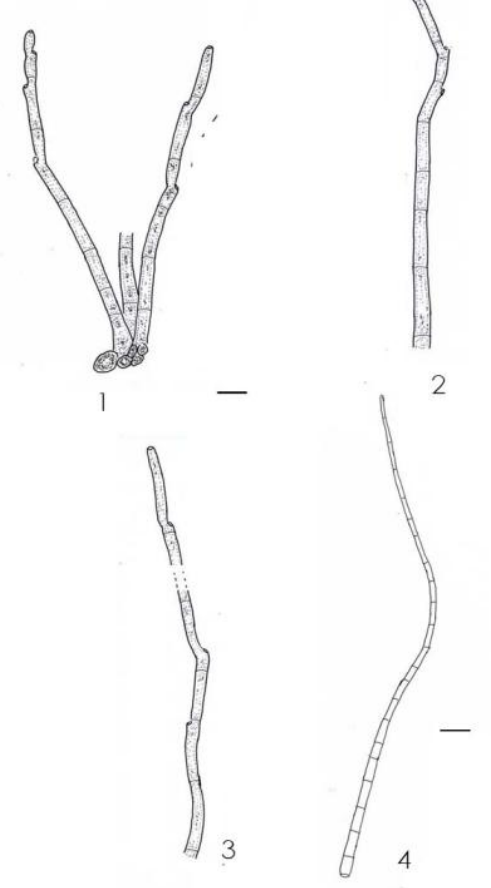

Fig. 40 - Cercospora sp. sp. 3 on Ziziphus sp. from leaf spots: 1 . Stroma with attached conidiophores. 2-3. Conidiophores. 4. Conidium. Bars: 1$4=10 \mu \mathrm{m}$.

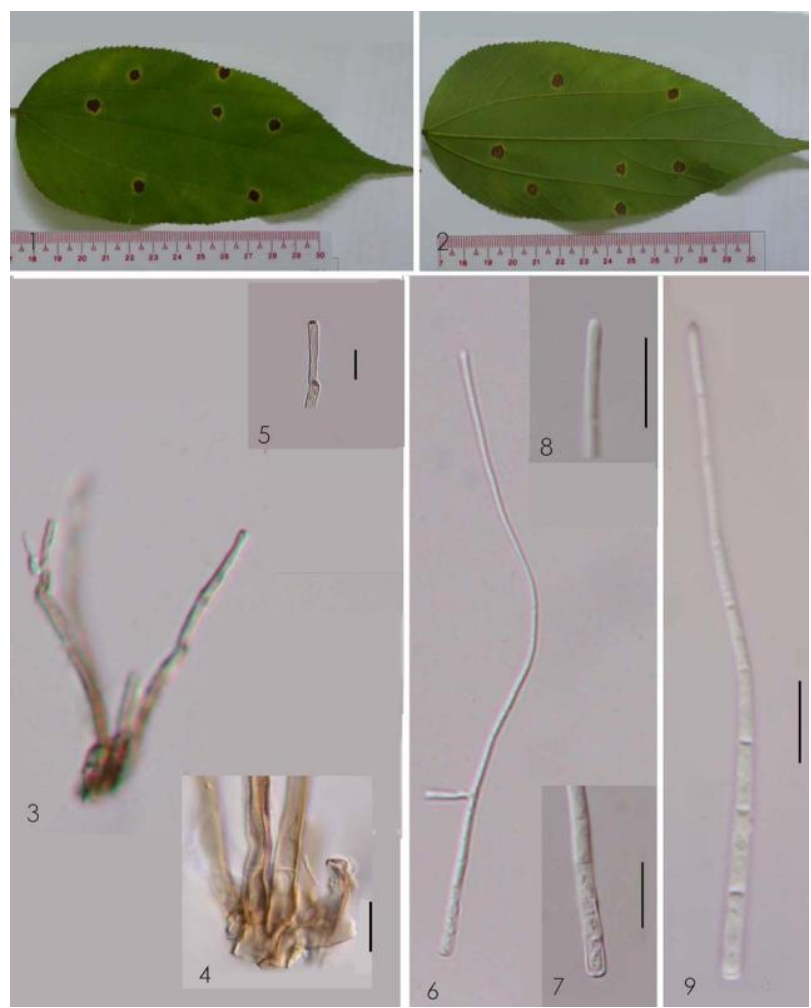

Fig. 41 - Cercospora sp. sp. 3 on Ziziphus sp. from leaf spots: 1-2. Leaf spots on host leaves (1. upper surface, 2. lower surface). 3. Stromata with attached conidiophores. 5. Stroma. 5. Apex of conidiophore. 6. Conidium. 7. Base of Conidium. 8. Apex of conidia. 9. Conidium. Bars: $1-2=10 \mathrm{~mm} .3-9=10 \mu \mathrm{m}$.

Cercospora tageteae Cif., Sydowia 8: 252, 1954.

Figs 42-43.

Leaf spots small to fairly large, suborbicular to irregular, 2-5 $\mathrm{mm}$ in diam., greybrown to dark brown in the centre, and with dark brown margin. Colonies amphigenous, scattered, dark brown. Mycelium internal; hyphae branched, $2.5-4 \mu \mathrm{m}$ wide $(\bar{x}=3.16$ $\mu \mathrm{m}, \mathrm{n}=7$ ), septate, constricted at the septa, distances between septa $8-15 \mu \mathrm{m}(\bar{x}=11.67$ $\mu \mathrm{m}, \mathrm{n}=7)$, brownish or green-hyaline, wall approximately $0.3-0.5 \mu \mathrm{m}$ wide $(\bar{x}=0.43 \mu \mathrm{m}$, $\mathrm{n}=7)$, smooth, forming plate-like plectenchymatous stromatic hyphal aggregations. Stromata developed, small to mediumsized, globular to subglobular, substomatal and intraepidermal, $10-20 \mu \mathrm{m}$ in diam. $(\bar{x}=16.5$ $\mu \mathrm{m}, \mathrm{n}=7$ ), dark brown to black in mass, composed of swollen hyphal cells, subglobose, rounded to angular in outline, $5-8 \mu \mathrm{m}$ wide $(\bar{x}$ $=6.7 \mu \mathrm{m}, \mathrm{n}=9$ ), brown to dark brown, wall 
$0.5-1 \mu \mathrm{m}$ wide $(\bar{x}=0.67 \mu \mathrm{m}, \mathrm{n}=9)$, smooth. Conidiophores fasciculate, arising from stromata (1-4 per fascicle), emerging through stomata, unbranched, straight to curved, cylindrical, $25-68 \times 5-6 \mu \mathrm{m}(\bar{x}=46 \times 5.33$ $\mu \mathrm{m}, \mathrm{n}=13), 0-1$-septate, distance between septa 8-38 $\mu \mathrm{m}(\bar{x}=16.4 \mu \mathrm{m}, \mathrm{n}=30)$, medium brown, paler at the apex, wall $0.5-0.8 \mu \mathrm{m}$ wide ( $\bar{x}=0.56 \mu \mathrm{m}, \mathrm{n}=30$ ), smooth, 0-2-times geniculate. Conidiogenous cells terminal, cylindrical, $12-38 \times 4-5 \mu \mathrm{m}(\bar{x}=25 \times 4.5 \mu \mathrm{m}$, $\mathrm{n}=8$ ), pale brown; conidiogenous loci conspicuous, subcircular, $1.5-3 \mu \mathrm{m}$ wide $(\bar{x}=$ $2.16 \mu \mathrm{m}, \mathrm{n}=8)$, wall $0.5-0.8 \mu \mathrm{m}$ thick $(\bar{x}=$ $0.65 \mu \mathrm{m}, \mathrm{n}=8$ ), thickened and darkened. Conidia solitary, acicular to obclavate, straight to curved, $45-54 \times 5-6 \mu \mathrm{m}(\bar{x}=48.8 \times 5.6$ $\mu \mathrm{m}, \mathrm{n}=5), 1-3$-septate, hyaline to subhyaline, thin-walled $0.25-0.3 \mu \mathrm{m}(\bar{x}=0.29 \mu \mathrm{m}, \mathrm{n}=5)$, smooth, tip acute, base obconically truncate; hila thickened and darkened $1.5-3 \mu \mathrm{m}$ wide $(\bar{x}$ $=2.6 \mu \mathrm{m}, \mathrm{n}=5$ ), wall of the hila $0.25-0.3 \mu \mathrm{m}$ $(\bar{x}=0.29 \mu \mathrm{m}, \mathrm{n}=5)$ thick.

(Asteraceae).

Known hosts - Tagetes patula L.
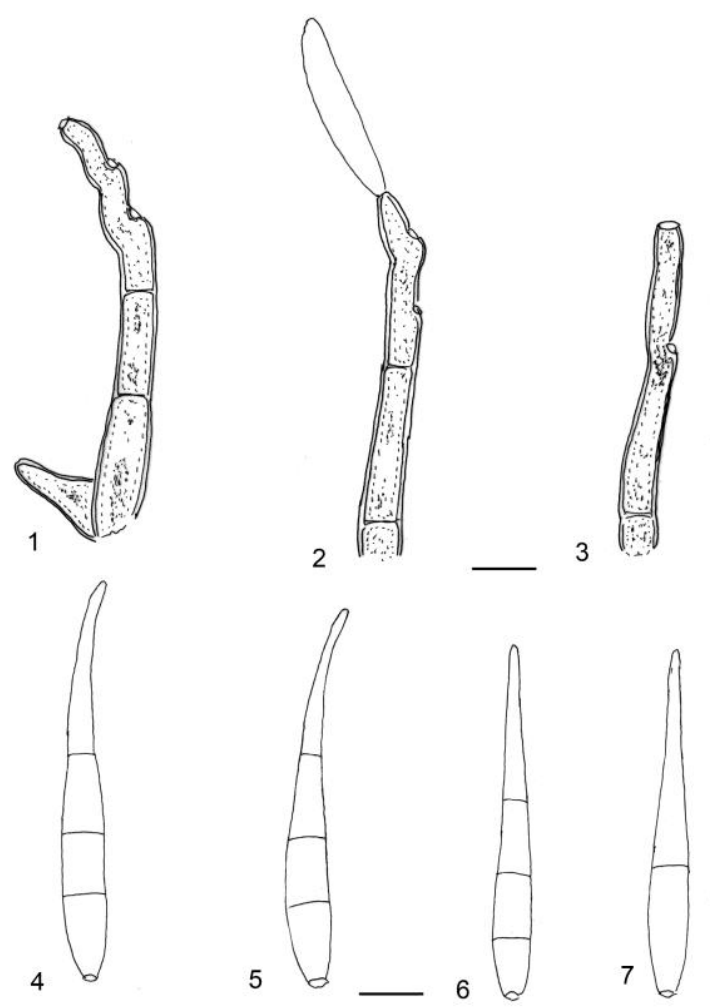

Fig. 42 - Cercospora tageteae on Tagetes patula from leaf spots: 1-3. Conidiophores. 4-7. Conidia. Bars: $1-7=10 \mu \mathrm{m}$

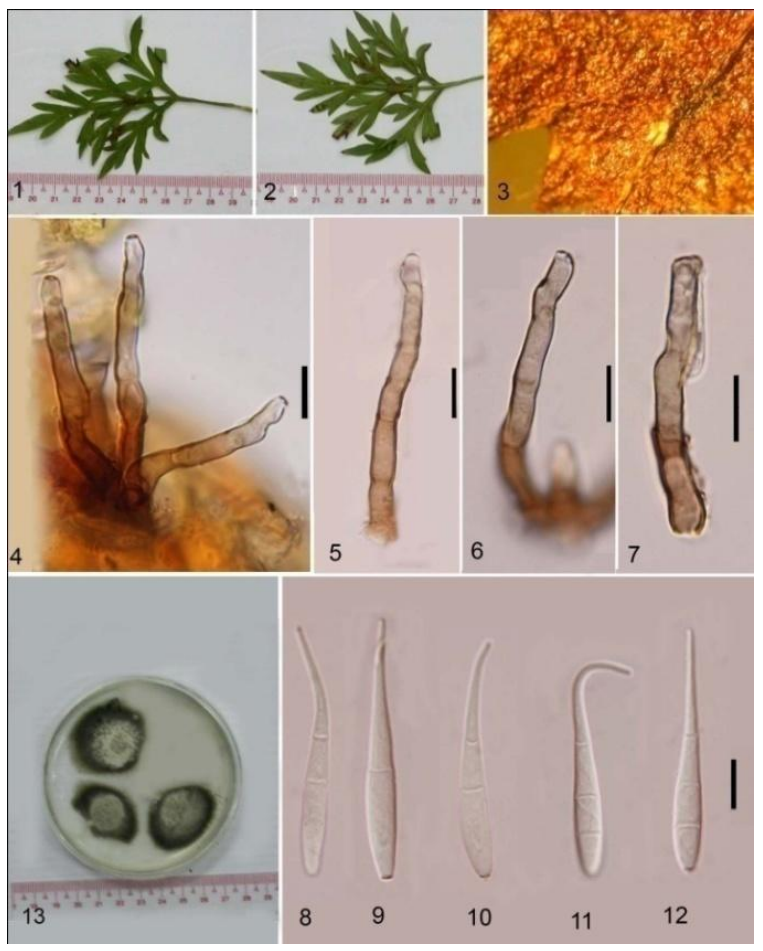

Fig. 43 - Cercospora tageteae on Tagetes patula from leaf spots: 1-2. Leaf spots on host leaves (1. upper surface, 2. lower surface). 3. Colonies. 4. Stroma with attached conidiophores. 5-7. Conidiophores. 9-12. Conidia. 13. Culture. Bars: $1-2=10 \mathrm{~mm}$. 3. Not to scale. $4-$ $12=10 \mu \mathrm{m} .13=10 \mathrm{~mm}$.

Known distribution - Asia: Thailand; North America and West Indies: Dominican Republ.

Material examined - Chiang Rai Province, Muang District, Sri Pangsang Village, on leaves of Tagetes patula (Asteraceae), 30 July 2010. P. Phengsintham (P609). GenBank accession no (ITS, KC677890).

Notes - A true Cercospora s. str. distinct from $C$. apii s. lat. by lacking leaf spots, having frequently branched conidiophores, small conidiogenous loci, 1-2.5 $\mu \mathrm{m}$ wide, and obclavate-cylindrical to acicular conidia (Crous \& Braun, 2003).

Literature - Crous \& Braun (2003: 396).

Cercospora verniciferae Chupp \& Viérgas, Bol. Soc. Bras. Agron. 8: 58, 1945. Figs 44-45.

Leaf spots small to medium, suborbicular to irregular, 1-4 $\mathrm{mm}$ in diam., brown in the centre, and with brown-yellow margin. Colonies hypophyllous, scattered, dark brown. Mycelium internal; hyphae branched, 3-5 $\mu \mathrm{m}$ 
wide ( $\bar{x}=3.57 \mu \mathrm{m}, \mathrm{n}=7$ ), septate, constricted at the septa, distance between septa 7-12 $\mu \mathrm{m}$ $(\bar{x}=8.42 \mu \mathrm{m}, \mathrm{n}=7$ ), brownish or greenhyaline, wall $0.5-0.8 \mu \mathrm{m}$ wide $(\bar{x}=0.54 \mu \mathrm{m}, \mathrm{n}$ $=7$ ), smooth, forming plate-like plectenchymatous stromatic hyphal aggregations. Stromata developed, small to medium-sized, globular to subglobular, substomatal and intraepidermal, $16-33 \mu \mathrm{m}$ in diam. ( $\bar{x}=23.6$ $\mu \mathrm{m}, \mathrm{n}=8$ ), dark brown to black in mass, composed of swollen hyphal cells, subglobose, rounded to angular in outline, 5-10 $\mu \mathrm{m}$ wide $(\bar{x}=7.9 \mu \mathrm{m}, \mathrm{n}=13)$, brown to dark brown, wall $0.5-0.8 \mu \mathrm{m}$ wide $(\bar{x}=0.68 \mu \mathrm{m}, \mathrm{n}=13)$, smooth. Conidiophores fasciculate, arising from stromata (1-4 per fascicle), emerging through stomata, unbranched, straight to curved, cylindrical, 45-89 $\times 5-7 \mu \mathrm{m}(\bar{x}=71.2$ $\times 5.4 \mu \mathrm{m}, \mathrm{n}=5$ ), $2-5$-septate, distance between septa 5-30 $\mu \mathrm{m}(\bar{x}=16.2 \mu \mathrm{m}, \mathrm{n}=16)$, medium brown, paler at the apex, wall $0.5-0.8 \mu \mathrm{m}$ wide ( $\bar{x}=0.63 \mu \mathrm{m}, \mathrm{n}=16$ ), smooth, 0-2-times geniculate. Conidiogenous cells integrated, terminal, cylindrical, $16-30 \times 4-5 \mu \mathrm{m}(\bar{x}=$ $24.5 \times 4.5 \mu \mathrm{m}, \mathrm{n}=4)$, pale brown; conidiogenous loci conspicuous, subcircular, $2-2.5 \mu \mathrm{m}$ wide $(\bar{x}=2.12 \mu \mathrm{m}, \mathrm{n}=4)$, wall $0.5-0.8 \mu \mathrm{m}$ thick $(\bar{x}=0.57 \mu \mathrm{m}, \mathrm{n}=4)$, thickened and darkened. Conidia solitary, acicular to obclavate, straight to curved, $23-105 \times 2-4 \mu \mathrm{m}$ $(\bar{x}=64 \times 2.8 \mu \mathrm{m}, \mathrm{n}=5), 5-12$-septate, hyaline to subhyaline, thin-walled $0.3-0.5 \mu \mathrm{m}(\bar{x}=$ $0.31 \mu \mathrm{m}, \mathrm{n}=5$ ), smooth; tip acute; base truncate to obconically truncate, hila thickened and darkened $1-1.5 \mu \mathrm{m}$ wide $(\bar{x}=1.1 \mu \mathrm{m}, \mathrm{n}=$ 5), wall of the hila $0.3-0.35 \mu \mathrm{m}(\bar{x}=0.31 \mu \mathrm{m}$, $\mathrm{n}=5$ ) thick.

Known hosts - Rhus vernicifera DC., Spondias dulcis Parkinson, S. pinnata (L.f.) Kurz (Anacardiaceae).

Known distribution - Asia: Thailand; Oceania: American Samoa; South America: Brazil.

Material examined - Chiang Rai Province, Muang District, Sri Pangsang Village, on leaves of Spondias pinnata (Anacardiaceae), 22 December 2009, P. Phengsintham (MFLU10-0313).

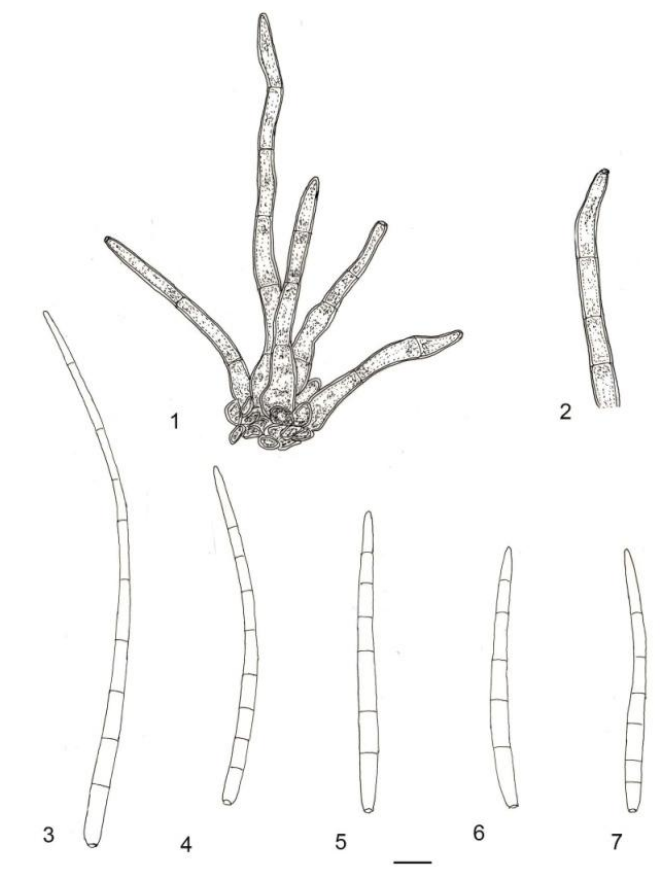

Fig. 44 - Cercospora verniciferae on Spondias pinnata from leaf spots: 1 . Stroma with attached conidiophores. 2. Conidiophore. 3-7. Conidia. Bars $=10 \mu \mathrm{m}$.

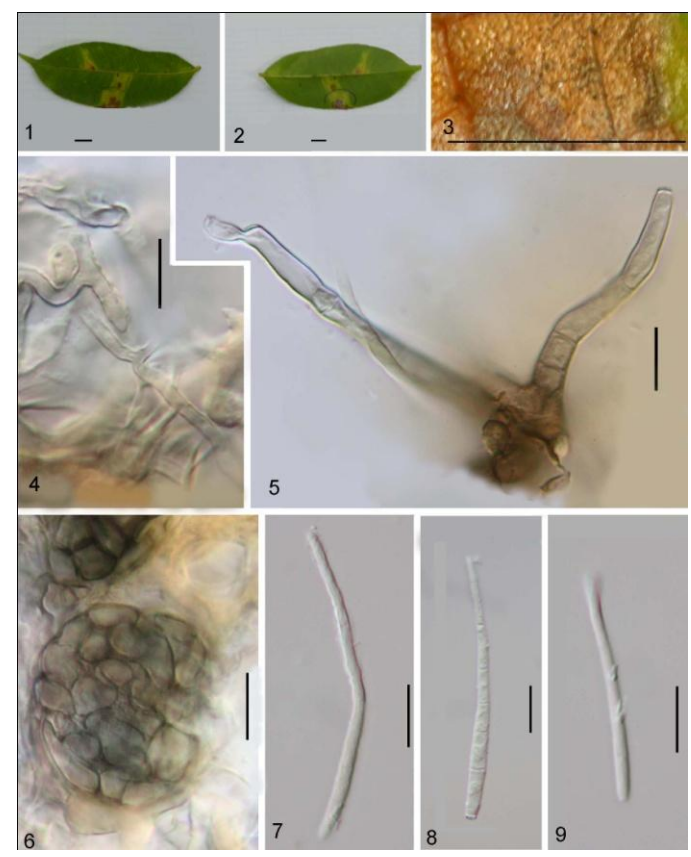

Fig. 45 - Cercospora verniciferae on Spondias pinnata from leaf spots: 1-2. Leaf spots on host leaves (1. upper surface, 2. lower surface). 3. Colonies. 4. Internal mycelium. 5. Stroma with attached conidiophores. 6. Stroma. 7-9. Conidia. Bars: $1-2=10 \mathrm{~mm} .3=3 \mathrm{~mm} .4-9=$ $10 \mu \mathrm{m}$. 
Notes - The collection from Thailand agrees well with $C$. verniciferae as circumscribed by Chupp (1954) with conidiophores 25-150 × 4.5-5 $\mu \mathrm{m}$ and conidia 35-175 $\times 2.5-$ $5 \mu \mathrm{m}$. C. verniciferae is part of the $C$. apii complex from which it is morphologically barely distinguishable (Crous \& Braun, 2003).

Literature - Chupp (1954: 44); Crous \& Braun (2003, 420).

Passalora barretoana (U. Braun \& Crous) D.J. Soares, U. Braun \& R.W. Barreto, Australas. Pl. Pathol. 35(3): 348, 2006. Figs (46-47).

$\equiv$ Passalora fusimaculans var. barretoana U. Braun \& Crous, in Crous and Braun, Mycosphaerella and its anamorphs: 1. Names published in Cercospora and Passalora. CBS Biodiversity Series 1: 453, 2003.

Leaf spots irregular, 3-8 mm diam., at first reddish brown, later becoming dark brown in the centre, brown yellowish margin. Colonies amphigenous, conspicuous. Mycelium internal; hyphae, branched, 3-4 $\mu \mathrm{m}$ wide $(\bar{x}=3.33 \mu \mathrm{m}, \mathrm{n}=7)$, septate, constricted at the septa, distance between septa $6-13 \mu \mathrm{m}(\bar{x}=$ $9.5 \mu \mathrm{m}, \mathrm{n}=7$ ), brownish to dark brown, wall 0.3-0.5 $\mu \mathrm{m}$ wide $(\bar{x}=0.46 \mu \mathrm{m}, \mathrm{n}=7$ ), smooth. Stromata developed, substomatal, subglobular, $14-42 \mu \mathrm{m}$ diam. $(\bar{x}=25.3 \mu \mathrm{m}, \mathrm{n}$ $=5$ ), brown to dark brown, stroma cells oval, ellipsoidal to angular in outline, $5-10 \mu \mathrm{m}$ wide $(\bar{x}=7.3 \mu \mathrm{m}, \mathrm{n}=15)$, dark brown, wall approximately $0.5-0.8 \mu \mathrm{m}$ wide $(\bar{x}=0.6 \mu \mathrm{m}$, $\mathrm{n}=15)$, smooth. Conidiophores fasciculate, arising from stromata stromata (2-19 per fascicle), unbranched, geniculate, cylindrical, straight to curved, $20-150 \times 3-9 \mu \mathrm{m}(\bar{x}=101$ $\times 5.88 \mu \mathrm{m}, \mathrm{n}=15), 4-8$-septate, distance between septa $10-30 \mu \mathrm{m}(\bar{x}=18.4 \mu \mathrm{m}, \mathrm{n}=$ 30 ), pale brown or olivaceous-brown; wall $0.5-$ $0.8 \mu \mathrm{m}$ wide $(\bar{x}=0.51 \mu \mathrm{m}, \mathrm{n}=30)$, smooth. Conidiogenous cells integrated, terminal, cylindrical, tapering to the apex, 10-30 × 3-6 $\mu \mathrm{m} \quad(\bar{x}=18.4 \times 4.38 \mu \mathrm{m}, \mathrm{n}=9)$; conidiogenous loci (scars) small, thickened and slightly darkened, $1.5-3 \mu \mathrm{m}$ diam. $(\bar{x}=2 \mu \mathrm{m}$, $\mathrm{n}=13$ ), wall of the loci approximately $0.5-0.8$ $\mu \mathrm{m}$ thick $(\bar{x}=0.53 \mu \mathrm{m}, \mathrm{n}=13)$, Conidia solitary or catenate, chains, frequently branched, acropleurogenous, simple, cylindrical with rounded ends or ellipsoidal, straight to curved, $20-57 \times 3-5 \mu \mathrm{m}(\bar{x}=40.62 \times 3.62$ $\mu \mathrm{m}, \mathrm{n}=13), 1-5$-septate, slightly constricted at the septa, very pale to mid pale brown or cream-brown, smooth, wall $0.3-0.5 \mu \mathrm{m}$ thick ( $\bar{x}=0.35 \mu \mathrm{m}, \mathrm{n}=13$ ), apex rounded, base long obconically truncate, hila $1.5-2 \mu \mathrm{m}$ wide $(\bar{x}=1.81 \mu \mathrm{m}, \mathrm{n}=13)$, wall of the hila $0.3-0.5$ $\mu \mathrm{m}$ wide $(\bar{x}=0.35 \mu \mathrm{m}, \mathrm{n}=13)$, darkened.

Known hosts - Echinochloa esculenta (A. Braun) H.Scholz, E. polystachya (Kunth) Hitchc., Hymenachne amplexicaulis (Rudge) Nees, Panicum sp. (Poaceae).

Known distribution - Asia: Thailand;

South America: Brazil.

Material examined - Chiang Rai Province, Muang District, Sri Pangsang Village, on leaves of Echinochloa esculenta (Poaceae), 15 September 2009, P. Phengsintham (MFLU10-0315) and Huay Kang Pah National Park, on leaves of Panicum sp. (Poaceae), 4 December 2009, P. Phengsintham (MFLU10-0316).

Notes - The collections from Sri Pangsang Village and from Huay Kang Pah National Park, Chiang Rai Province agree well with the original description of Passalora barretoana (三 P. fusimaculans var. barretoana) in Crous \& Braun (2003).

Literature - Crous \& Braun (2003: 192).

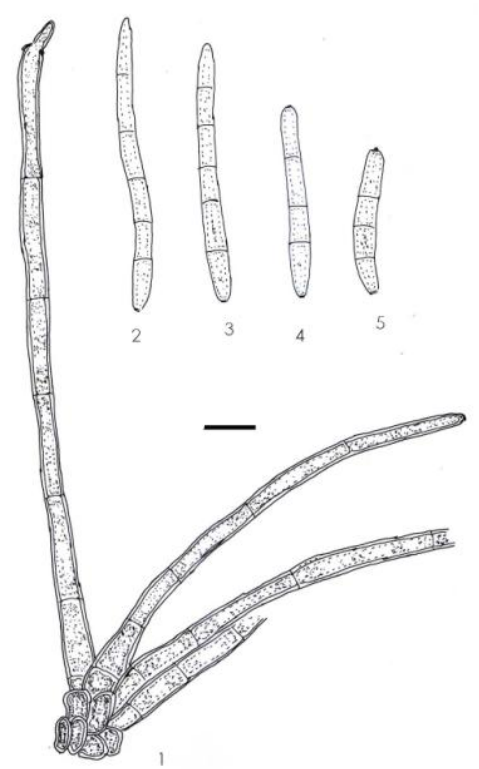

Fig. 46 - Passalora barretoana on Panicum sp. from leaf spots: 1. Stroma with attached conidiophores. 2-6. Conidia. Bars: $1-6=10$ $\mu \mathrm{m}$. 


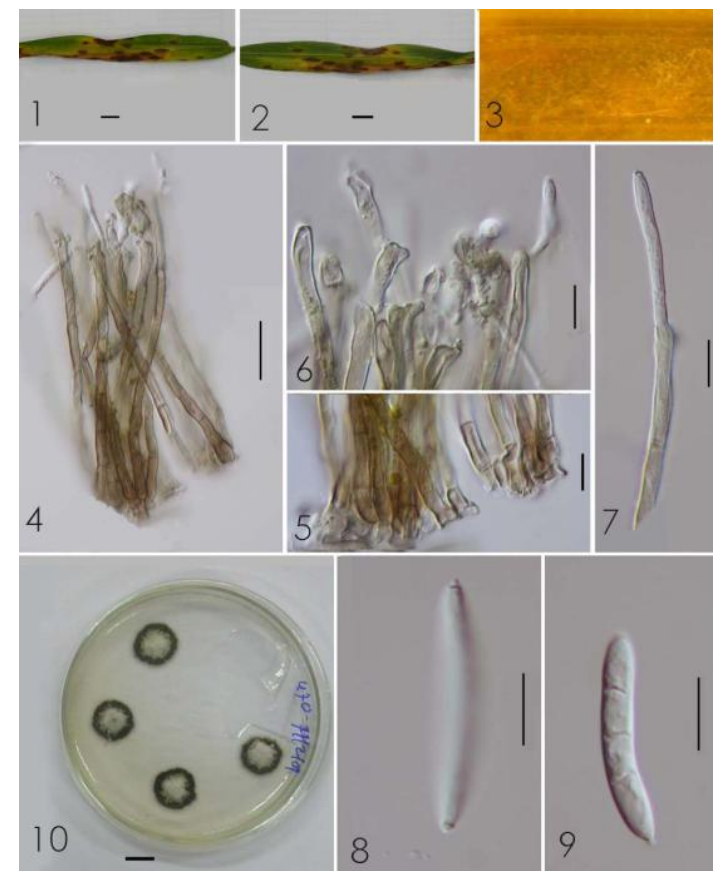

Fig. 47 - Passalora barretoana on Panicum sp. from leaf spots: 1. Leaf spots on host leaves (1. upper surface, 2. lower surface). 3. Colonies. 4. Stroma with attached conidiophores. 5. Stroma. 6. Apices of Conidiophores. 8-9. Conidia. 10. Culture. Bars: $1-2=10 \mathrm{~mm}$. 3. Not to scale. $4-9=10 \mu \mathrm{m} .10=10 \mathrm{~mm}$.

Passalora broussonetiae (Goh \& W.H. Hsieh) U. Braun \& Crous, in Crous and Braun, Mycosphaerella and its anamorphs: 1. Names published in Cercospora and Passalora. CBS Biodiversity Series 1: 515, 2003. Figs 48-49. Leaf spots irregular, 1-9 mm diam., at first reddish brown, later becoming dark brown in the centre, grey to reddish brown margin. Colonies amphigenous, conspicuous. Mycelium external; external hyphae, branched, 2-4 $\mu \mathrm{m}$ wide $(\bar{x}=2.77 \mu \mathrm{m}, \mathrm{n}=13)$, septate, constricted at the septa, distance between septa 17-38 $\mu \mathrm{m}(\bar{x}=28.46 \mu \mathrm{m}, \mathrm{n}=13)$, brownish to dark brown, wall $0.3-0.5 \mu \mathrm{m}$ wide $(\bar{x}=0.44$ $\mu \mathrm{m}, \mathrm{n}=13$ ), smooth. Stromata not developed. Conidiophores single, born on external mycelium, unbranched or branched, geniculate, cylindrical, straight to curved, $170-390 \times 2-5$ $\mu \mathrm{m}(\bar{x}=303 \times 3 \mu \mathrm{m}, \mathrm{n}=5), 5-17$-septate, distance between septa $13-40 \mu \mathrm{m}(\bar{x}=27 \mu \mathrm{m}$, $\mathrm{n}=30$ ), pale brown or olivaceous-brown; wall 0.3-0.5 $\mu \mathrm{m}$ wide $(\bar{x}=0.47 \mu \mathrm{m}, \mathrm{n}=30$ ), smooth. Conidiogenous cells integrated, terminal, cylindrical, tapering to the apex, 10$21 \times 4-5 \mu \mathrm{m}(\bar{x}=16,3 \times 4.18 \mu \mathrm{m}, \mathrm{n}=11)$; conidiogenous loci (scars) small, thickened and slightly darkened, $1-2 \mu \mathrm{m}$ diam. $(\bar{x}=1.40 \mu \mathrm{m}$, $\mathrm{n}=11$ ), wall of the loci approximately $0.3-0.5$ $\mu \mathrm{m}$ thick $(\bar{x}=0.40 \mu \mathrm{m}, \mathrm{n}=11)$. Conidia solitary or catenate, chains, frequently branched, acropleurogenous, simple, cylindrical with rounded ends or ellipsoidal, straight to curved, $6-28 \times 4-6 \mu \mathrm{m}(\bar{x}=20.5 \times 5.3 \mu \mathrm{m}$, $\mathrm{n}=30$ ), 0-3-septate, slightly constricted at the septa, very pale to mid pale brown or creambrown, smooth, wall $0.3-0.5 \mu \mathrm{m}$ thick $(\bar{x}=$ $0.47 \mu \mathrm{m}, \mathrm{n}=30$ ), apex rounded ends, based long obconically truncate, hila $1-2 \mu \mathrm{m}$ wide $(\bar{x}$ $=1.45 \mu \mathrm{m}, \mathrm{n}=30$ ), wall of the hila $0.3-0.5 \mu \mathrm{m}$ wide $(\bar{x}=0.47 \mu \mathrm{m}, \mathrm{n}=30)$, darkened.

Colonies on PDA after 3 weeks at $25^{\circ} \mathrm{C}$ cream to dark cream-brown in the centre, margin cream brown, reaching 4-6 $\mathrm{mm}$ diam., hyphae $2-6 \mu \mathrm{m}$ wide $(\bar{x}=3.1 \mu \mathrm{m}, \mathrm{n}=30)$, septate, slightly constricted at the septa, distance between septa 9-40 $\mu \mathrm{m}(\bar{x}=24.47$ $\mu \mathrm{m}, \mathrm{n}=30)$, primary mycelium cream to dark brown, but the second and following ones cream to brownish, wall smooth. Conidia solitary or catenate, chains, frequently branched, acropleurogenous, simple, cylindrical with rounded ends or ellipsoidal, straight to curved, $17-41 \times 4-5 \mu \mathrm{m}(\bar{x}=26.1 \times 4.38$ $\mu \mathrm{m}, \mathrm{n}=21), 1-2$-septate, slightly constricted at the septa, very pale to mid pale brown or cream-brown, smooth, wall $0.3-0.5 \mu \mathrm{m}$ thick $(\bar{x}=0.46 \mu \mathrm{m}, \mathrm{n}=21)$, apex rounded ends, based long obconically truncate, hila 1-2 $\mu \mathrm{m}$ wide $(\bar{x}=1.5 \mu \mathrm{m}, \mathrm{n}=8)$, wall of the hila $0.3-$ $0.5 \mu \mathrm{m}$ wide $(\bar{x}=0.46 \mu \mathrm{m}, \mathrm{n}=8)$, darkened. In the culture, crystal Seminole-red occurring.

Known host - Broussonetia papyrifera (L.) L'Hér. ex Vent. (Moraceae).

Known distribution - Asia: Taiwan, Thailand.

Material examined - Chiang Rai Province, Wiang Chiang Rung District, Tadsak waterfall, on leaves of Broussonetia papyrifera (Moraceae), 23 December 2009, P. Phengsintham (MFLU10-0314). GenBank accession no (ITS, KC677892).

Notes - The collection MFLU10-0314 from Tadsak waterfall, Chiang Rai province agrees well with Passalora broussonetiae (Crous \& Braun, 2003), but the hyphae are smooth to distinctly verruculose.

Literature - Crous \& Braun (2003). 


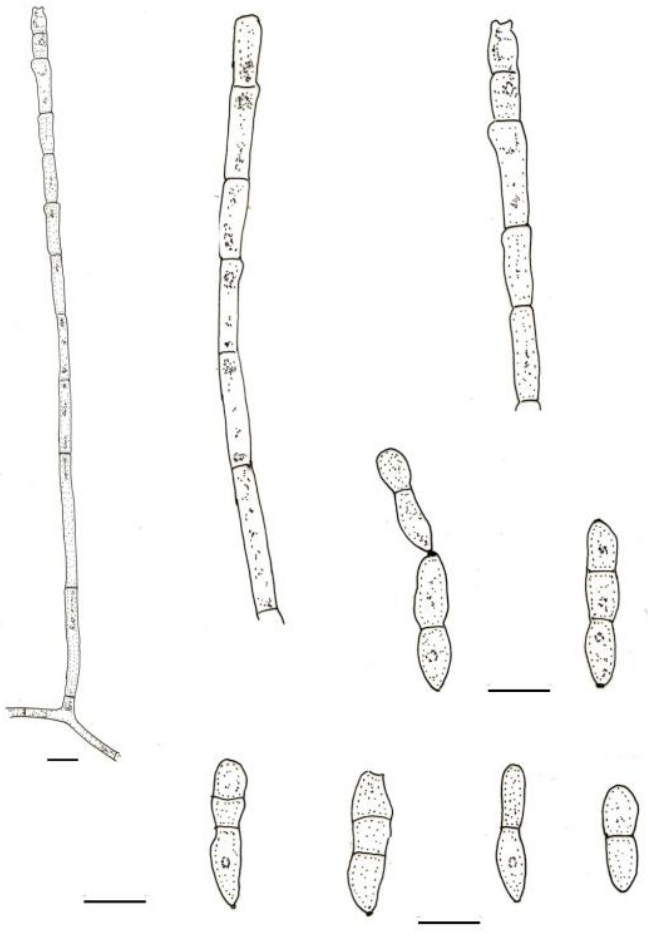

Fig. 48 - Passalora broussonetiae on Broussonetia papyrifera: 1. External hypha with attached conidiophore. 2-3. Apices of conidiophores. 4-9. Conidia. Bars: $1-9=10$ $\mu \mathrm{m}$.

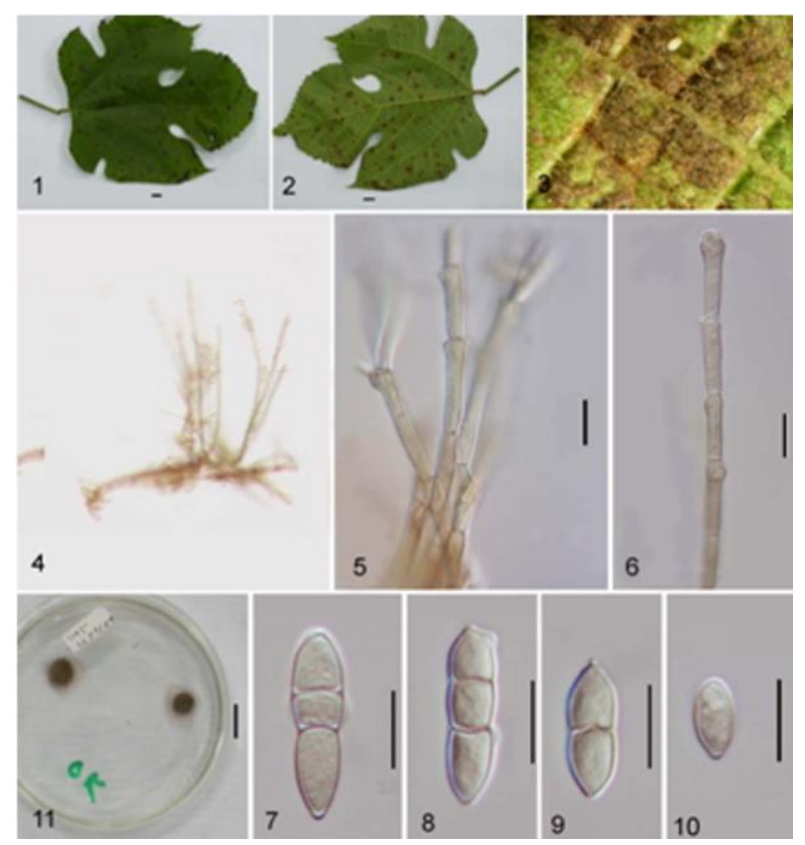

Fig. 49 - Passalora broussonetiae on Broussonetia papyrifera on host leaves: 1-2. Leaf spots on host leaves (1. upper surface, 2. lower surface). 3. Colonies. 4. External mycelium with attached conidiophores. 5-6. Conidiophores. 7-10. Conidia. 11. Culture. Bars: $1=10 \mathrm{~mm}, 4-10=10 \mu \mathrm{m}, 11=10 \mathrm{~mm}$.
Passalora fusimaculans (G.F. Atk.) U. Braun $\&$ Crous, in Crous and Braun, Mycosphaerella and its anamorphs: 1. Names published in Cercospora and Passalora. CBS Biodiversity Series 1: 515, 2003.

Figs 50-51. $\equiv$ Cercospora fusimaculans G.F. Atk., Elisha Mitchell Sci. Soc. 8:50, 1892.

$\equiv$ Phaeoramularia fusimaculans (G.F. Atk.) X.J. Liu \& Y.L. Guo, Acta Phytopathol. Sin. 12: 9, 1982.

= Cercospora agrostis G.F. Atk., J. Elisha Mitchell Sci. Soc. 8: 44, 1892.

$=$ Cercospora panici Davis, Trans. Wiscosin Acad. Soc. 19: 714, 1919.

$\equiv$ Cercosporina panici (Davis) Sacc., Syll. Fung. 25: 904, 1931.

= Cercospora panici-milliacei Sawada, Rep. Gov. Agric. Res. Inst. Formosa 51: 131, 1931.

Leaf spots circular to irregular, $1-5 \mathrm{~mm}$ diam., reddish brown to medium brown in the centre, and with a brown to dark brown margin. Colonies amphigenous, scattered. Mycelium internal, inconspicuous. Stromata developed, substomatal, subglobular, $20-50 \mu \mathrm{m}$ diam. $(\bar{x}$ $=34.4 \mu \mathrm{m}, \mathrm{n}=8$ ), brown to dark brown, stroma cells oval, ellipsoidal to angular in outline, $4-5 \mu \mathrm{m}$ wide $(\bar{x}=4.6 \mu \mathrm{m}, \mathrm{n}=12)$, dark brown, wall $0.3-0.5 \mu \mathrm{m}$ wide $(\bar{x}=0.33$ $\mu \mathrm{m}, \mathrm{n}=12$ ), smooth. Conidiophores fasciculate, arising from stromata (6-24 per fascicle), erect, straight or curved, unbranched, $10-52 \times 3-5 \mu \mathrm{m}(\bar{x}=24.2 \times 4 \mu \mathrm{m}, \mathrm{n}=12), 0$ 1-septate, distance between septa 8-30 $\mu \mathrm{m}(\bar{x}$ $=16.9 \mu \mathrm{m}, \mathrm{n}=16$ ), pale to moderately olivaceous-brown, wall $0.3-0.5 \mu \mathrm{m}$ wide $(\bar{x}=$ $0.48 \mu \mathrm{m}, \mathrm{n}=16$ ), smooth. Conidiogenous cells integrated, $10-30 \times 3-5 \mu \mathrm{m}(\bar{x}=17.1 \times 4 \mu \mathrm{m}$, $\mathrm{n}=10)$, apex $1.5-2 \mu \mathrm{m}$ wide $(\bar{x}=1.8 \mu \mathrm{m}, \mathrm{n}=$ 6), wall approximately $0.3-0.5 \mu \mathrm{m}$ thick $(\bar{x}=$ $0.42 \mu \mathrm{m}, \mathrm{n}=6$ ), subtruncate, cicatrized, pale olivaceous or brown; conidiogenous loci conspicuous, $1.5-2 \mu \mathrm{m}$ wide $(\bar{x}=1.8 \mu \mathrm{m}, \mathrm{n}=6)$, wall $0.3-0.5 \mu \mathrm{m}$ wide $(\bar{x}=0.43 \mu \mathrm{m}, \mathrm{n}=6)$, smooth. Conidia solitary or caternate, cylindrical, straight to moderately curved, 18$38 \times 1.5-2 \mu \mathrm{m}(\bar{x}=30.25 \times 1.9 \mu \mathrm{m}, \mathrm{n}=8), 3-$ 4-septate, slightly constricted at the septa, pale olivaceous, wall $0.2-0.3 \mu \mathrm{m}$ wide $(\bar{x}=0.25$ $\mu \mathrm{m}, \mathrm{n}=8$ ), smooth or finely verruculose, both ends subtruncate when catenulate, bluntly rounded at the apex in solitary and primary 
conidia, apical hila $0.5-0.8 \mu \mathrm{m}$ wide $(\bar{x}=0.75$ $\mu \mathrm{m}, \mathrm{n}=5)$, wall $0.3-0.5 \mu \mathrm{m}(\bar{x}=0.35 \mu \mathrm{m}, \mathrm{n}=$ 5) thick, with subtruncate base, basal hila $0.5-$ $1.5 \mu \mathrm{m}$ wide $(\bar{x}=0.76 \mu \mathrm{m}, \mathrm{n}=5)$, wall $0.3-$ $0.5 \mu \mathrm{m}(\bar{x}=0.34 \mu \mathrm{m}, \mathrm{n}=5)$ thick.

Known hosts - of species of Agrostis, Brachiaria, Beckeropsis, Chasmopodium, Digitaria, Echinochloa, Eleusine, Entolasia, Ichnanthus, Leptoloma, Oplismenus, Panicum, Paspalidium, Pennisetum, Rottboellia, Setaria, Sorghum, Stenotaphrum, Urochloa, Zea (Poaceae).

Known distribution - Africa: Botswana, Ethiopia, Ghana, Guinea, Ivory Coast, Kenya, Malawi, Nigeria, Rwanda, Sierra Leone, South Africa, Sudan, Tanzania, Togo, Uganda, Zambia, Zimbabwe; Asia: Brunei, China, India, Japan, Malaysia, Philippines, Taiwan, Thailand; Europe: Azerbaijan, France, Georgia; North America and West Indies: Costa Rica, Cuba, Dominican Rep., El Salvador, Guatemala, Honduras, Jamaica, Mexico, Nicaragua, Panama, Trinidad and Tobago, USA (AL, PL, IA, ID, KS, NC, ND, OK, OR, TX, VA, WI); Australia; Oceana: Fiji, New Zealand, Palau, Papua New Guinea, Samoa, Solomon Islands, Vanuatu; South America: Bolivia, Brazil, Colombia, Ecuador, Guyana, Peru, Venezuela.

Material examined - Chiang Rai Province, Doi Tung National Park, on leaves of Agrostis sp. (Poaceae), 18 August 2009, P. Phengsintham (MFLU10-0317).

Notes - The collection from Doi Tung National Park, Chiang Rai Province agrees well with Passalora fusimaculans as circumscribed by Chupp (1954), Vasudeva (1963), Ellis (1976) and Hsieh \& Goh (1990).

Literature - Chupp (1954: 246); Vasudeva (1963: 112); Ellis (1976: 260); Hsieh \& Goh (1990: 141); Crous \& Braun (2003: 203).

Pseudocercospora atromarginalis (G.F. Atk.) Deighton, Mycol. Pap. 140: 139, 1976.

Figs 52-53. $\equiv$ Cercospora atromarginalis G.F. Atk., J. Elisha Mitchel Sci. Soc. 8: 59, 1892.

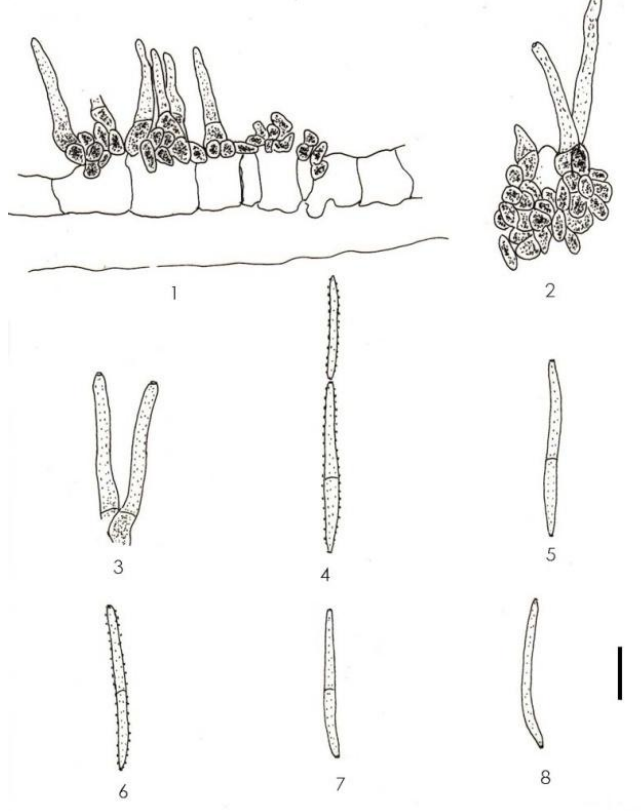

Fig. 50 - Passalora fusimaculan on Agrostis sp.: 1-2. Stromata with attached conidiophores. 3. Conidiophores. 4-8. Conidia. Bars: $1-8=10 \mu \mathrm{m}$.

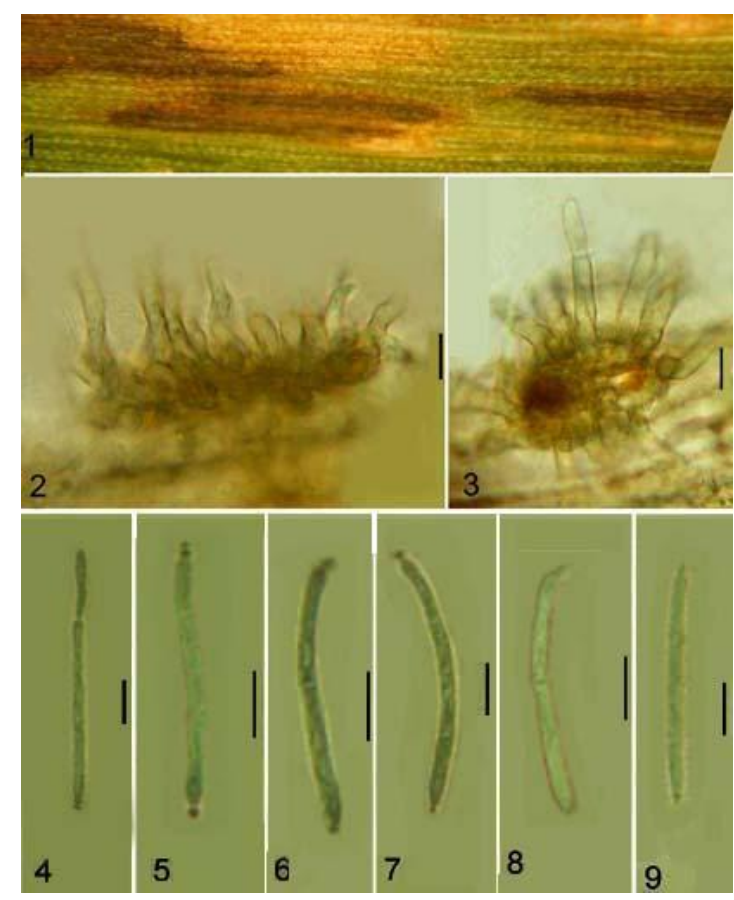

Fig. 51 - Passalora fusimaculan on Agrostis sp. from leaf spots: 1. Leaf spots on host leaf (upper surface). 2-3. Stromata with attached conidiophores. 4-9. Conidia. Bars: $1=10 \mathrm{~mm}$. $2-9=10 \mu \mathrm{m}$. 
= Cercospora solani-biflori Sawada, Formosan Agric. Rev. 39: 701, 1942, nom. inval.

Leaf spots suborbicular or angular, 1$12 \mathrm{~mm}$ diam., at first yellowish and later becoming pale brown or grey to brown in the centre, and with yellowish margin. Colonies amphigenous, but chiefly hypophyllous. Mycelium internal, inconspicuous. Stromata developed, oval to ellipsoidal, $12-15 \mu \mathrm{m}$ diam., stromatal cells oval, ellipsoidal to angular in outline, 5-6 $\mu \mathrm{m}$ wide, dark brown, wall approximately $0.5 \mu \mathrm{m}$ wide, smooth. Conidiophores fasciculate, arising from stromata (1-5 per fascicle), erect, straight or curved, simple or branched, apex obtuse, 35$115 \times 3.5-5 \mu \mathrm{m}(\bar{x}=59.2 \times 4.03 \mu \mathrm{m}, \mathrm{n}=11)$, 1-11-septate, distance between septa 7-35 $\mu \mathrm{m}$ $(\bar{x}=16 \mu \mathrm{m}, \mathrm{n}=30$ ), pale to medium brown; wall $0.3-0.5 \mu \mathrm{m}$ wide $(\bar{x}=0.49 \mu \mathrm{m}, \mathrm{n}=30$ ), smooth. Conidiogenous cells integrated, apex obtuse, $10-35 \times 3-5 \mu \mathrm{m}(\bar{x}=18.6 \times 4.15 \mu \mathrm{m}$, $\mathrm{n}=12$ ), pale olivaceous or brown; conidiogenous loci inconspicuous. Conidia solitary, subcylindrical, slightly obclavate-cylindrical, or sometimes slightly clavate-cylindrical, almost straight or slightly to strongly curved, $32-55 \times 3.5-6 \mu \mathrm{m}(\bar{x}=40.5 \times 4.10 \mu \mathrm{m}, \mathrm{n}=$ 12), 3-5-septate, slightly constricted at the septa, pale olivaceous, wall 0.3-0.5 $\mu \mathrm{m}$ wide $(\bar{x}=0.32 \mu \mathrm{m}, \mathrm{n}=12)$, smooth, broadly rounded at the apex, the basal cell rounded at the base or more abruptly tapering towards the base, hilum 1.5-2 $\mu \mathrm{m}$ wide, wall $0.3-0.5 \mu \mathrm{m}$ wide, unthickened, not darkened.

Known hosts - numerous Solanum ssp. and Lycianthes biflora (Lour.) Bitter (Solanaceae).

Known distribution - widespread (see Crous \& Braun 2003).

Material examined - Chiang Rai Province, Doi Tung National Park, on leaves of Lycianthes biflora [ $\equiv$ Solanum biflorum] (Solanaceae), 22 August 2009, P. Phengsintham (P439).

Notes - Cercospora solani-biflori, described from Taiwan on Solanum biflorum ( $\equiv$ Lycianthes biflora), is an invalid name, which was reduced to synonymy with $C$. atromarginalis $[\equiv P$. atromarginalis $]$ by Chupp (1954) and Goh \& Hsieh (1990).
Literature - Chupp (1954: 532); Hsieh \& Goh (1990: 322), Crous \& Braun (2003).

Pseudocercospora balsaminae (Syd.)

Deighton, Mycol. Pap. 140: 139, 1976.

Figs 54-55.

$\equiv$ Cercoseptoria balsaminae Syd., Ann. Mycol. 33: 69, 1935.

Leaf spots subcircular to irregular, 2-15 $\mathrm{mm}$ diam., at first yellowish, later becoming brown in the center, brown to yellowish at the margin. Colonies hypophyllous, scattered, conspicuous. Mycelium internal; hyphae branched, $2-9 \mu \mathrm{m}$ wide $(\bar{x}=6 \mu \mathrm{m}, \mathrm{n}=7)$, septate, constricted at the septa, distance between septa 5-27 $\mu \mathrm{m}(\bar{x}=15.5 \mu \mathrm{m}, \mathrm{n}=7)$, subhyaline to brownish, wall $0.3-1 \mu \mathrm{m}$ wide $(\bar{x}=0.65 \mu \mathrm{m}, \mathrm{n}=7)$, smooth, forming platelike plectenchymatous stromatic hyphal aggregations. Stromata oval to ellipsoidal, 15$30 \mu \mathrm{m}$ diam. $(\bar{x}=22.6 \mu \mathrm{m}, \mathrm{n}=6)$, brown to dark brown, stroma cells oval, ellipsoidal and angular, 3-7 $\mu \mathrm{m}$ wide $(\bar{x}=5 \mu \mathrm{m}, \mathrm{n}=10)$, dark brown, wall $0.5-0.8 \mu \mathrm{m}$ wide $(\bar{x}=0.65 \mu \mathrm{m}, \mathrm{n}$ $=10)$, smooth. Conidiophores fasciculate, arising from stromata (9-30 per fascicle), geniculate, unbranched, $9-16 \times 2-4 \mu \mathrm{m}(\bar{x}=$ $12.35 \times 3.05 \mu \mathrm{m}, \mathrm{n}=15), 0-1$-septate, slightly constricted at the septa, distance between septa 4-12 $\mu \mathrm{m}(\bar{x}=7.64 \mu \mathrm{m}, \mathrm{n}=15)$, uniformly pale to medium brown, much paler and narrower toward the tip, wall $0.3-0.5 \mu \mathrm{m}(\bar{x}=$ $0.47 \mu \mathrm{m}, \mathrm{n}=15)$, smooth. Conidiogenous cells terminal, $7-12 \times 2-4 \mu \mathrm{m}(\bar{x}=9.64 \times 2.70 \mu \mathrm{m}$, $\mathrm{n}=14$ ), obtuse; conidiogenous loci inconspicuous, unthickened, not darkened. Conidia solitary, obclavate, straight to slightly curved, $32-44 \times 2-4 \mu \mathrm{m}(\bar{x}=38.52 \times 2.64 \mu \mathrm{m}, \mathrm{n}=$ 13), 3-5-septate, pale olivaceous-brown, wall $0.3-0.5 \mu \mathrm{m}$ wide $(\bar{x}=0.36 \mu \mathrm{m}, \mathrm{n}=13)$, smooth, tip subacute, base obconically truncate, hila $1-2 \mu \mathrm{m}$ wide $(\bar{x}=1.39 \mu \mathrm{m}, \mathrm{n}=$ 13).

Colonies on PDA after 3 weeks at $25^{\circ} \mathrm{C}$ dark grey, reaching 20-25 mm diam., hyphae 2-9 $\mu \mathrm{m}$ wide $(\bar{x}=9 \mu \mathrm{m}, \mathrm{n}=20)$, septate, constricted at the septa, distance between septa 5-27 $\mu \mathrm{m}(\bar{x}=15.25 \mu \mathrm{m}, \mathrm{n}=20)$, brownish or subhyaline, wall $0.3-1 \mu \mathrm{m}$ wide $(\bar{x}=0.65 \mu \mathrm{m}$, $\mathrm{n}=20$ ), smooth. Conidia not formed in culture.

Known hosts - Impatiens balsamina L. (Balsaminaceae). 
Known distribution - Asia: Brunei, China, India, Korea, Thailand.

Material examined - Chiang Rai Province, Muang District, Sri Pangsang Village, on leaves of Impatiens balsamina (Balsaminaceae), 20 July 2010, P. Phengsintham (MFLU10-0404).

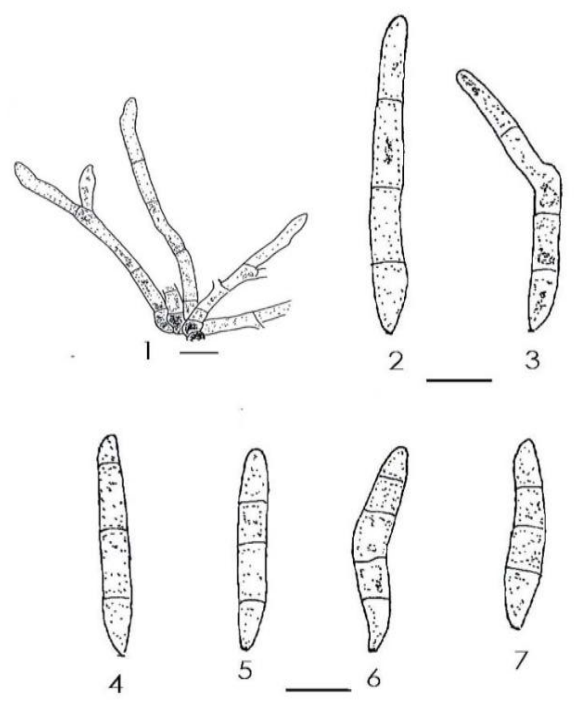

Fig. 52 - Pseudocercospora atromarginalis on Lycianthes biflora: 1. Stroma with attached conidiophores. 2-7. Conidia. Bar: 1-7 = $10 \mu \mathrm{m}$.

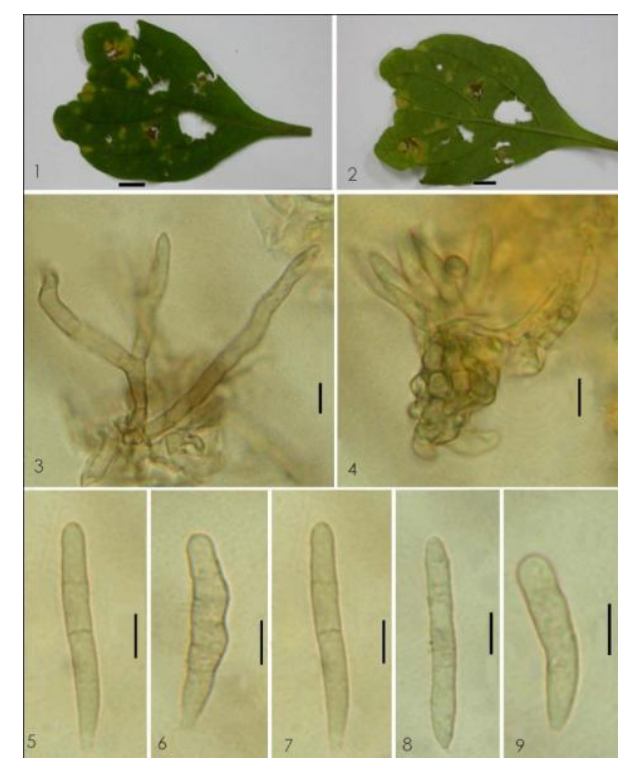

Fig. 53 - Pseudocercospora atromarginalis on Lycianthes biflora from leaf spots: 1-2. Leaf spots on host leaves (1. upper surface, 2. lower surface). 3-4. Stromata with attached conidiophores. 5-9. Conidia. Bar: $1-2=10$ mm. 3-9 = $10 \mu \mathrm{m}$.
Notes - Pseudocercospora balsaminae is a variable species with a wide range of conidial length. Guo \& Hsieh (1995) described conidiophores of $6.5-40 \times 2.5-4 \mu \mathrm{m}$ and conidia $25-90 \times 1.5-3 \mu \mathrm{m}$. Shin \& $\operatorname{Kim}(2001)$ even described conidiophores up to $56 \times 4 \mu \mathrm{m}$ and conidia up to $105 \times 3.5 \mu \mathrm{m}$. The conidiophores and conidia in the collection from Thailand are relatively short, but they are within the variation of this species. $P$. balsaminicola (J.M. Yen \& Lim) U. Braun \& Crous (Yen \& Lim 1980, Crous \& Braun 2003) is a similar confusable species, but clearly differentiated by its hyaline acicular-filiform, basally truncate, long and narrow conidia 45$132 \times 2-2.5 \mu \mathrm{m}$. P. nojimae (Togashi \& Katsuki) Y.L. Guo \& X.J. Liu was considered a possible synonym of $P$. balsaminae by Deighton (1976), but represents a distinct species with much broader conidia (Guo \& Hsieh 1995, Crous \& Braun 2003).

Literatures - Yen \& Lim (1980: 152); Guo \& Hsieh (1995: 33); Shin \& Kim (2001: 166).
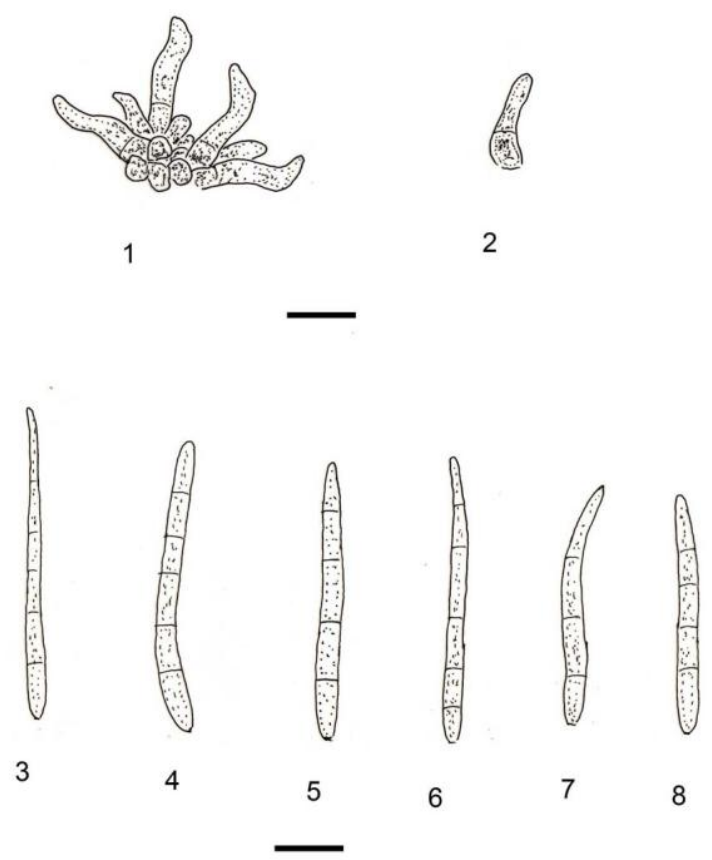

Fig. 54 - Pseudocercospora balsaminae on Impatiens balsamina: 1. Stroma with attached conidiophores. 2. Conidiophore. 3-8. Conidia. Bars: $=10 \mu \mathrm{m}$. 


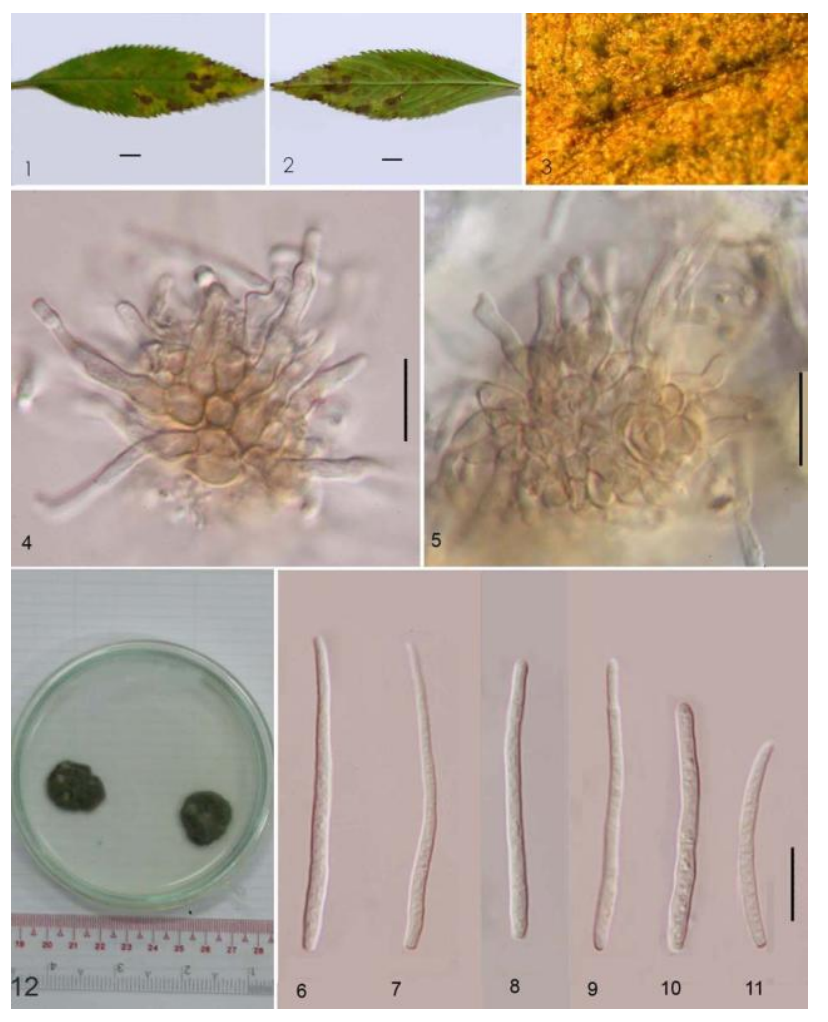

Fig. 55 - Pseudocercospora balsaminae on Impatiens balsamina from leaf spots: 1-2. Leaf spots on host leaves (1. upper surface and 2. lower surface). 3. Colonies. 4-5. Stromata with attached conidiophores. 6-11. Conidia. 12. Culture. Bars: 1, 2, $12=10 \mathrm{~mm} .4-11=10$ $\mu \mathrm{m}$. 3. Not to scale.

Pseudocercospora bischofiae (W. Yamam.) Deighton, Trans. Brit. Mycol. Soc. 88: 388, 1987.

Figs 56-57.

三 Cercospora bischofiae W. Yamam., Trans. Sapporo Nat. Hist. Soc. 13: 139, 1934.

$\equiv$ Pseudocercospora bischofiae (W. Yamam.) Goh \& W.H. Hsieh, Trans. Mycol. Soc. Republ. China 2: 114, 1987.

Leaf spots subcircular to irregular, 1-5 $\mathrm{mm}$ diam., at first yellowish, later becoming brown in the center, dark brown at the margin. Colonies hypophyllous, scattered, conspicuous. Mycelium internal and external; internal hyphae inconspicuous; external hyphae branched, $2-3 \mu \mathrm{m}$ wide $(\bar{x}=2.5 \mu \mathrm{m}, \mathrm{n}=5)$, septate, constricted at the septa, distance between septa 9-14 $\mu \mathrm{m}(\bar{x}=11.5 \mu \mathrm{m}, \mathrm{n}=5)$, brownish, subhyaline, wall $0.5-0.8 \mu \mathrm{m}$ wide $(\bar{x}=0.65 \mu \mathrm{m}, \mathrm{n}=5)$, smooth, forming platelike plectenchymatous stromatic hyphal aggregations. Stromata oval to ellipsoidal, 5$17 \mu \mathrm{m}$ diam. $(\bar{x}=11 \mu \mathrm{m}, \mathrm{n}=3)$, brown to dark brown, stromatal cells oval, ellipsoidal and angular, $2-6 \mu \mathrm{m}$ wide $(\bar{x}=3.8 \mu \mathrm{m}, \mathrm{n}=15)$, dark brown, wall $0.5-0.8 \mu \mathrm{m}$ wide $(\bar{x}=0.56$ $\mu \mathrm{m}, \mathrm{n}=15$ ), smooth. Conidiophores singly or fasciculate, arising from stromata (1-2 per fascicle) and borne on external hyphae, geniculate, unbranched, 9-24 × 2-4 $\mu \mathrm{m}(\bar{x}=$ $15 \times 3.25 \mu \mathrm{m}, \mathrm{n}=9), 0-2$-septate, slightly constricted at the septa, distance between septa 4-11 $\mu \mathrm{m}$ long $(\bar{x}=7.25 \mu \mathrm{m}, \mathrm{n}=20)$, uniformly pale to medium brown, much paler and more narrow toward the tip, wall $0.5-0.8$ $\mu \mathrm{m} \quad(\bar{x}=0.61 \mu \mathrm{m}, \mathrm{n}=20)$, smooth. Conidiogenous cells terminal, 6-11 $\times 2-4 \mu \mathrm{m}$ $(\bar{x}=8.25 \times 3 \mu \mathrm{m}, \mathrm{n}=7)$, obtuse, conidiogenous loci inconspicuous, unthickened, not darkened. Conidia solitary, obclavate, straight to slightly curved, 41-56 $\times$ 2-3 $\mu \mathrm{m}(\bar{x}=50.04 \times 2.6 \mu \mathrm{m}, \mathrm{n}=11), 4-6-$ septate, pale olivaceous-brown, wall $0.3-0.5$ $\mu \mathrm{m}$ wide $(\bar{x}=0.34 \mu \mathrm{m}, \mathrm{n}=11)$, smooth, tip subacute, base truncate, hila $0.72-2 \mu \mathrm{m}$ wide $(\bar{x}=1.44 \mu \mathrm{m}, \mathrm{n}=11)$.
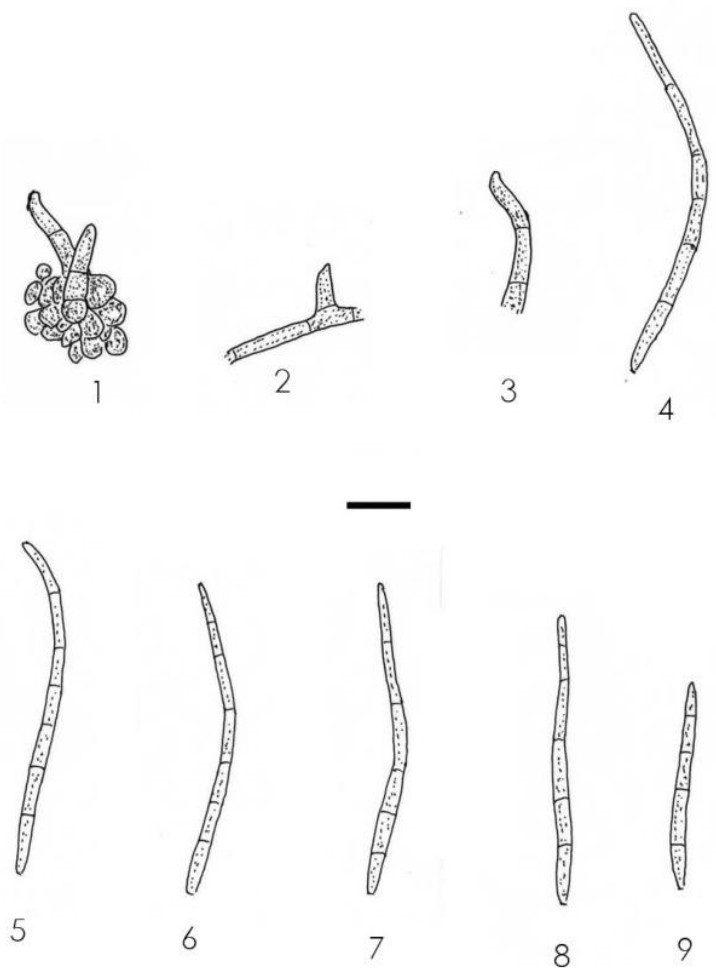

Fig. 56 - Pseudocercospora bischofiae on Bischofia javanica from leaf spots: 1. Stroma with attached conidiophores. 2. External hyphae. 3. Conidiophore. 4-9. Conidia. Bars: $1-9=10 \mu \mathrm{m}$. 


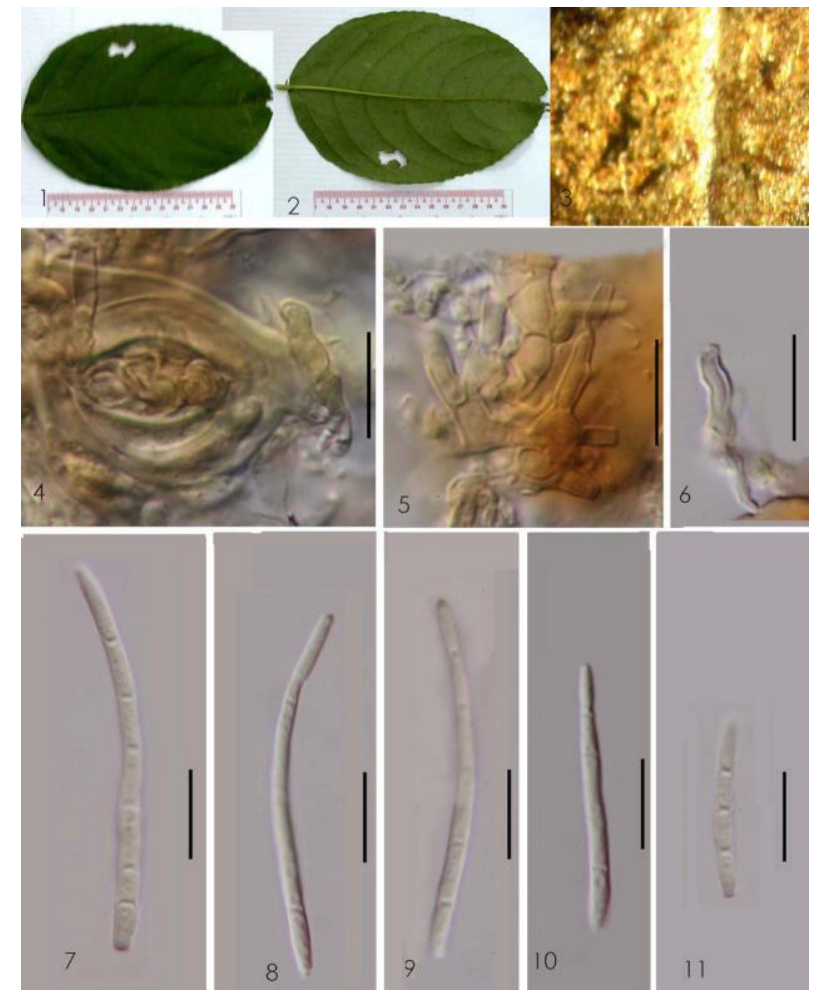

Fig. 57 - Pseudocercospora bischofiae on Bischofia javanica from leaf spots: 1-2. Leaf spots on host leaves (1. upper surface, 2. lower surface). 3. Colonies. 4-5. Stromata with attached conidiophores. 6. Conidiophore. 7-11. Conidia. Bars: $1-2=10 \mathrm{~mm}$. 3. Not to scale. $4-11=10 \mu \mathrm{m}$.

Known hosts - Bischofia javanica Blume, B. trifoliata (Roxb.) Hook (Phyllanthaceae $=$ Euphorbiaceae $)$.

Known distribution - Asia: China, Hong Kong, Taiwan, Thailand; North America: USA (FL).

Material examined - Chiang Rai Province, Muang District, Sri Pangsang Village, on leaves of Bischofia javanica (Phyllanthaceae), 22 August 2010, P. Phengsintham (P619). GenBank accession no (ITS, KC677896; LSU, KC677928).

Notes - The collection from Thailand agrees with the collection from Taiwan described by Hsieh \& Goh (1990) [conidiophores 10-70 $\times 3-5 \mu \mathrm{m}$ and conidia $20-80 \times 2-4 \mu \mathrm{m}]$ in having similar size of conidiophores and conidia.

Literature - Chupp (1954: 213); Hsieh \& Goh (1990: 120); Guo \& Hsieh (1995: 102); Guo et al. (1998: 118); Crous \& Braun (2003: 81).
Pseudocercospora carbonacea (L.E. Miles) N. Pons \& B. Sutton, Mycol. Pap. 160: 26, 1988.

Figs 58-59.

$\equiv$ Cercospora carbonacea L.E. Miles, Trans. Illinois Acad. Sci. 10: 255, 1917.

Leaf spots subcircular to irregular, 2-27 $\mathrm{mm}$ diam., at first yellowish brown, dark brown in the centre, brown to yellowish at the margin. Colonies amphigenous, conspicuous, scattered, grey. Mycelium internal; Hyphae branched, $1.5-4 \mu \mathrm{m}$ wide $(\bar{x}=2.15 \mu \mathrm{m}, \mathrm{n}=$ $5)$, septate, constricted at the septa, distance between septa 5-9 $\mu \mathrm{m}(\bar{x}=7 \mu \mathrm{m}, \mathrm{n}=5)$, brownish, subhyaline, wall $0.3-0.5 \mu \mathrm{m}$ wide $(\bar{x}=0.43 \mu \mathrm{m}, \mathrm{n}=5)$, smooth, forming platelike plectenchymatous stromatic hyphal aggregations. Stromata oval to ellipsoidal, 15$36 \mu \mathrm{m}$ diam. $(\bar{x}=23.67 \mu \mathrm{m}, \mathrm{n}=6)$, brown to dark brown, stromatal cells oval, ellipsoidal to angular, 4-9 $\mu \mathrm{m}$ wide $(\bar{x}=6.47 \mu \mathrm{m}, \mathrm{n}=10)$, dark brown, wall $0.5-0.8 \mu \mathrm{m}$ wide $(\bar{x}=0.65$ $\mu \mathrm{m}, \mathrm{n}=10)$, smooth. Conidiophores fasciculate, arising from stromata (2-4 per fascicle), not geniculate, unbranched, $12-26 \times 4-5 \mu \mathrm{m}$ $(\bar{x}=15.99 \times 4.41 \mu \mathrm{m}, \mathrm{n}=9), 0-2$-septate, slightly constricted at the septa, distance between septa $5-15 \mu \mathrm{m}$ long $(\bar{x}=8.95 \mu \mathrm{m}, \mathrm{n}=$ 10 ), uniformly pale to medium brown, much paler and narrower towards the tip, wall $0.3-$ $0.5 \mu \mathrm{m}(\bar{x}=0.48 \mu \mathrm{m}, \mathrm{n}=15)$, smooth. Conidiogenous cells terminal, 6-12 $\times 3-4 \mu \mathrm{m}$ $(\bar{x}=8.67 \times 3.52 \mu \mathrm{m}, \mathrm{n}=10)$, apex obtuse, conidiogenous loci inconspicuous, unthickened, not darkened. Conidia solitary, obclavate, straight to slightly curved, 17-99 $\times$ 2-4 $\mu \mathrm{m}(\bar{x}=70.58 \times 3.05 \mu \mathrm{m}, \mathrm{n}=10), 2-6-$ septate, pale olivaceous-brown, wall $0.3-0.5$ $\mu \mathrm{m}$ wide $(\bar{x}=0.36 \mu \mathrm{m}, \mathrm{n}=10)$, smooth, tip subacute, base obconically truncate, hila 1-2 $\mu \mathrm{m}$ wide $(\bar{x}=1.51 \mu \mathrm{m}, \mathrm{n}=10)$.

Known hosts - Dioscorea alata L., D. bulbifera L., D. caucasica Lipsky, $D$. cayenensis Lam., $D$. deltoidea Wall. ex Griseb., D. dumetorum (Kunth) Pax, D. gillettii Milne-Redh., D. glabra Roxb., D. nipponica Makino, D. oppositifolia L., D. spinosa Burm., D. trifida L. f., Dioscorea sp. (Dioscoreaceae).

Known distribution - Africa: Ethiopia, Ghana, Guinea, Nigeria, Sierra Leone, Tanzania, Togo; Asia: India, Indonesia, Myanmar, Thailand; North America and 
West Indies: Barbados, Canada, Cuba, Dominican Republ., French Antilles, Grenada, Haiti, Jamaica, Panama, Puerto Rico, Saint Lucia, Saint Vincent and the Grenadines, Virgin Islands, Trinidad and Tobago; South America: Brazil, Venezuela.

Material examined - Chiang Rai Province, Muang District, Sri Pangsang Village, on leaves of Dioscorea bulbifera (Dioscoreaceae), 16 August 2010, P. Phengsintham (P615). GenBank accession no (ITS, KC677897).

Notes - The collection from Thailand differs from Pseudocercospora carbonacea described by Hsieh \& Goh (1990) and Ellis (1976) in having distinctly geniculate conidiophores.

Litarature - Saccardo (1931: 874); Chupp (1954: 1960); Vasudeva (1963: 68); Ellis (1976: 257); Pons \& Sutton (1988: 26); Crous \& Braun (2003: 104).
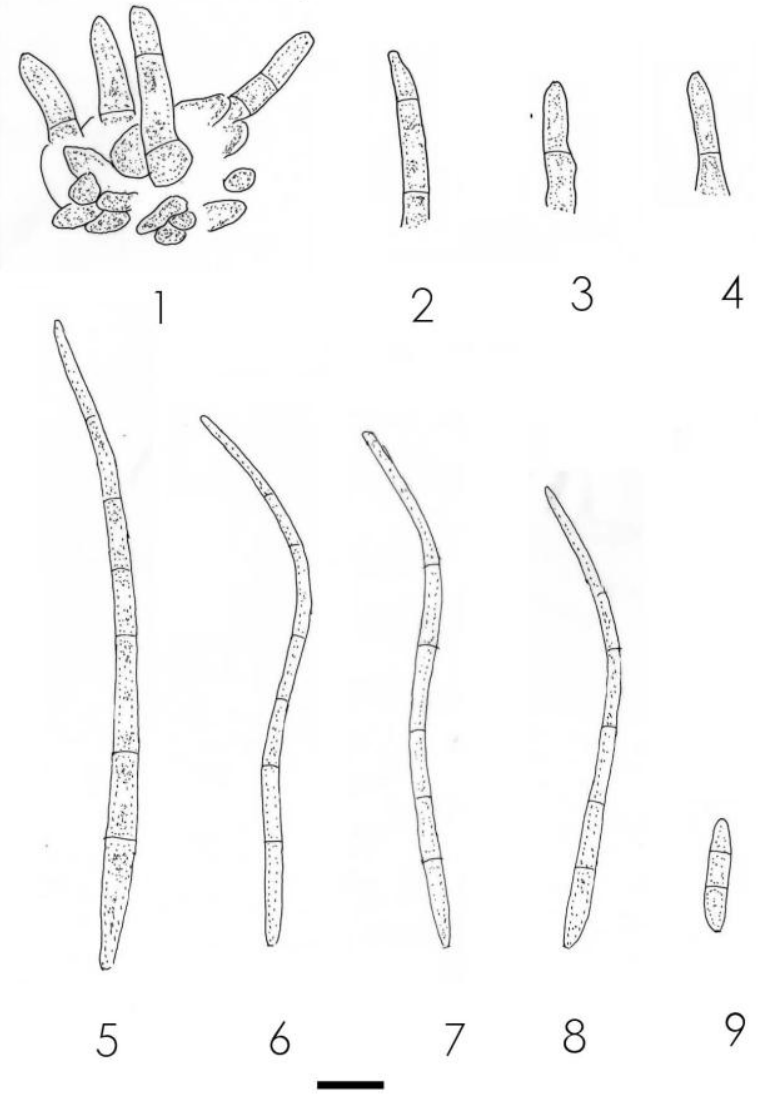

Fig. 58 - Pseudocercospora carbonacea on Dioscorea bulbifera: 1. Stroma with attached conidiophores. 2-4. Conidiophores. 5-9. Conidia. Bars: $1-9=10 \mu \mathrm{m}$.
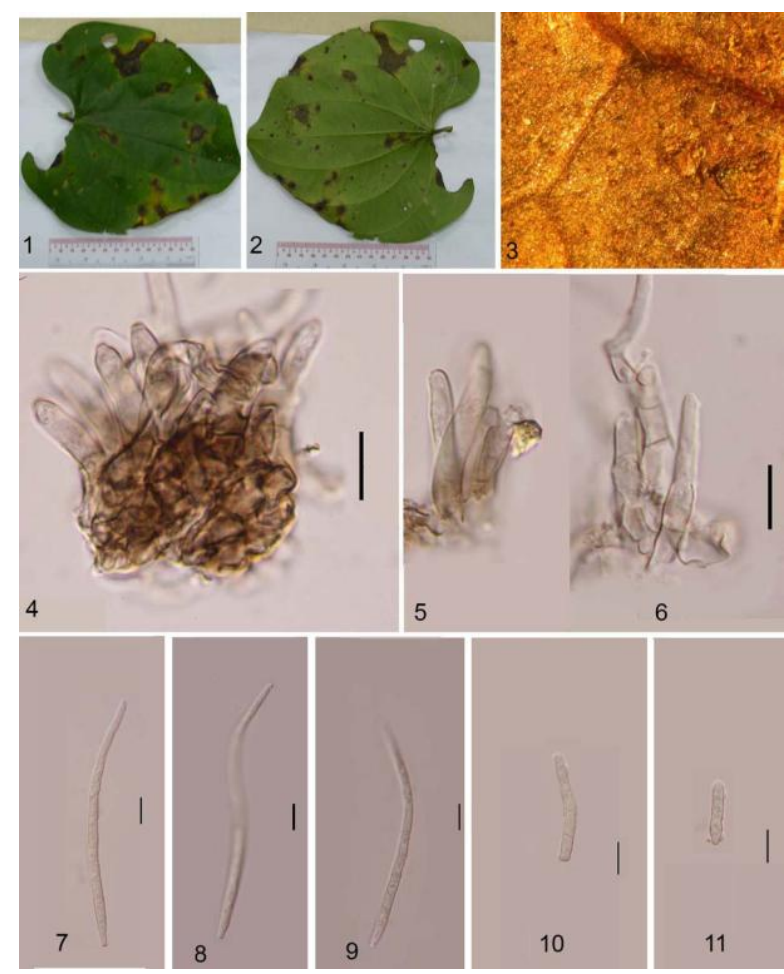

Fig. 59 - Pseudocercospora carbonacea on Dioscorea bulbifera from leaf spots: 1-2. Leaf spots on host leaves (1. upper surface, 2. lower surface). 3. Colonies. 4-6. Stromata with attached conidiophores. 7-11. Conidia. Bars: $1-2=10 \mathrm{~mm}$. 3. Not to scale. $4-11=10 \mu \mathrm{m}$.

Pseudocercospora christellae Phengsintham, McKenzie, K.D. Hyde \& U. Braun, Mycosphere 1(3): 207, 2011. Figs 60-61. Leaf spots subcircular to irregular, 3-9 $\mathrm{mm}$ diam., at first brownish, later becoming brown, brown to dark yellowish brown at the margin. Colonies epiphyllous, conspicuous. Mycelium internal; hyphae branched, 2-3 $\mu \mathrm{m}$ wide $(\bar{x}=2.5 \mu \mathrm{m}, \mathrm{n}=5)$, septate, constricted at the septa, distance between septa $6-8 \mu \mathrm{m}(\bar{x}$ $=4 \mu \mathrm{m}, \mathrm{n}=5$ ), subhyaline to brownish, wall $0.3-0.5 \mu \mathrm{m}$ wide $(\bar{x}=0.4 \mu \mathrm{m}, \mathrm{n}=5)$, smooth, forming plate-like plectenchymatous stromatic hyphal aggregations. Stromata oval to ellipsoidal, $22-40 \mu \mathrm{m}$ diam. $(\bar{x}=28.5 \mu \mathrm{m}, \mathrm{n}=$ 7), brown to dark brown, stroma cells oval, ellipsoidal and angular, 3-10 $\mu \mathrm{m}$ wide $(\bar{x}=$ $7.66 \mu \mathrm{m}, \mathrm{n}=30$ ), dark brown, wall $0.5-0.8 \mu \mathrm{m}$ wide $(\bar{x}=0.63 \mu \mathrm{m}, \mathrm{n}=30)$, smooth. Conidiophores fasciculate, arising from stromata (3-16 per fascicle), geniculate, unbranched, $9-14 \times 2-4 \mu \mathrm{m}(\bar{x}=10.58 \times 3.05$ $\mu \mathrm{m}, \mathrm{n}=13), 0-1$-septate, slightly constricted at 
the septa, distance between septa 3-12 $\mu \mathrm{m}(\bar{x}$ $=8.8 \mu \mathrm{m}, \mathrm{n}=15$ ), uniformly pale to medium brown, paler and narrower towards the tip, wall $0.3-0.5 \mu \mathrm{m}$ wide $(\bar{x}=0.47 \mu \mathrm{m}, \mathrm{n}=15)$, smooth. Conidiogenous cells terminal, $8-12 \times$ $2-4 \mu \mathrm{m}(\bar{x}=9.99 \times 2.77 \mu \mathrm{m}, \mathrm{n}=8)$, obtuse; conidiogenous loci inconspicuous, unthickened, not darkened. Conidia solitary, obclavate, straight to slightly curved, 53-105 $\times$ 2-4 $\mu \mathrm{m}(\bar{x}=81.36 \times 2.90 \mu \mathrm{m}, \mathrm{n}=17), 3-9-$ septate, pale olivaceous-brown, wall $0.3-0.5$ $\mu \mathrm{m}$ wide $(\bar{x}=0.36 \mu \mathrm{m}, \mathrm{n}=17)$, smooth, tip subacute, base obconically truncate, hila 1-2 $\mu \mathrm{m}$ wide $(\bar{x}=1.81 \mu \mathrm{m}, \mathrm{n}=17)$.

Colonies on PDA after 3 weeks at $25^{\circ} \mathrm{C}$ dark grey, reaching $10-15 \mathrm{~mm}$ diam., hyphae 2-7 $\mu \mathrm{m}$ wide $(\bar{x}=4.25 \mu \mathrm{m}, \mathrm{n}=20)$, septate, constricted at the septa, distance between septa 9-19 $\mu \mathrm{m}(\bar{x}=14 \mu \mathrm{m}, \mathrm{n}=20)$, brownish or subhyaline, wall $0.3-0.8 \mu \mathrm{m}$ wide $(\bar{x}=0.52$ $\mu \mathrm{m}, \mathrm{n}=20$ ), smooth. Conidia not formed in culture.

Known host - Christella parasitica (L.) H. Lév. (Thelypteridaceae).

Known distribution - Asia: Thailand.

Material examined - Chiang Rai Province, Muang District, Mae Chan Village, on leaves of Christella parasitica (Thelypteridaceae), 18 July 2010. P. Phengsintham (MFLU10-0405, holotype). GenBank accession no (ITS, KC677898).

Notes - This is the first record of a Pseudocercospora on a host of the genus Christella. There are numerous Pseudocercospora spp. on ferns, but $P$. christellae differs from all species with fasciculate conidiophores in having very short conidiophores, viz. P. arachnioidis Y.L. Guo, $P$. athyri W.H. Hsieh \& Goh, P. cyatheae C. Nakash. \& Ivana, P. lonchitidis (Chupp) U. Braun \& Crous, P. nephlolepidis R. Kirschner, $P$. plagiogyiae Goh \& W.H. Hsieh, $P$. pteridophytophila Goh \& W.H. Hsieh, and $P$. thelypteridis Goh \& W.H. Hsieh (Chupp 1954, Hsieh and Goh 1990, Guo \& Hsieh 1995, Crous \& Braun 2003, Nakashima et al. 2007 (a), Kirschner \& Yen 2007). Other species are distinguished by forming superficial mycelium with solitary conidiophores, viz. $P$. abacopteridicola (J.M. Yen \& Lim) J.M. Yen, $P$. adianthi (Syd.) Deighton, $P$. lygodii Goh \& W.H. Hsieh, P. rumohrae W.H. Hsieh \& Goh (Chupp 1954, Hsieh \& Goh 1990, Yen \& Lim 1980). Four Pseudocercospora spp. on hosts of the Thelypteridaceae have been described: $P$. abacopteridicola on Abacopteris urophylla (with superficial mycelium and solitary conidiophores), P. phyllitidis (H.H. Home) U. Braun \& Crous, e.g. on Thelypteris tetragona (conidiophores up to $100 \mu \mathrm{m}$ long, conidia 3-5 $\mu \mathrm{m}$ wide), P. pteridophytophila on Cyclosorus acuminatus (conidiophores up to $50 \mu \mathrm{m}$ long, conidia shorter and narrower, 30-70 × 1-1.5 $\mu \mathrm{m}$ ), and $P$. thelypteridis (conidiophores up to $60 \mu \mathrm{m}$ long, conidia acicular-filiform) [Chupp 1954, Hsieh \& Goh 1990, Yen \& Lim 1980].

Literature - Chupp (1954); Hsieh \& Goh (1990); Guo \& Hsieh (1995); Yen \& Lim (1980: 151-163); Crous \& Braun (2003); Nakashima et al. (2007: 48-52); Kirschner \& Yen (2007: 219-237).
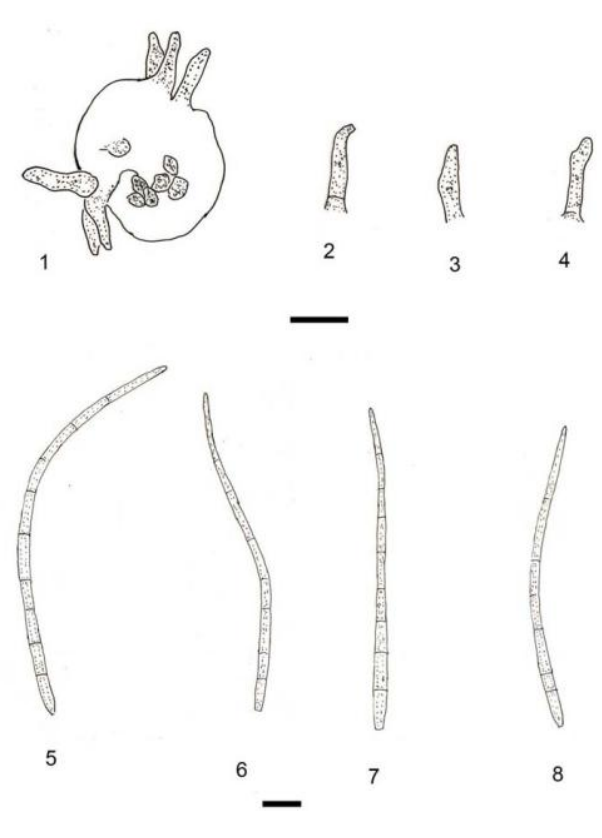

Fig. 60 - Pseudocercospora christellae on Christella parasitica: 1. Stroma with attached conidiophores. 2-4. Conidiophores. 5-8. Conidia. Bars: $=10 \mu \mathrm{m}$. 


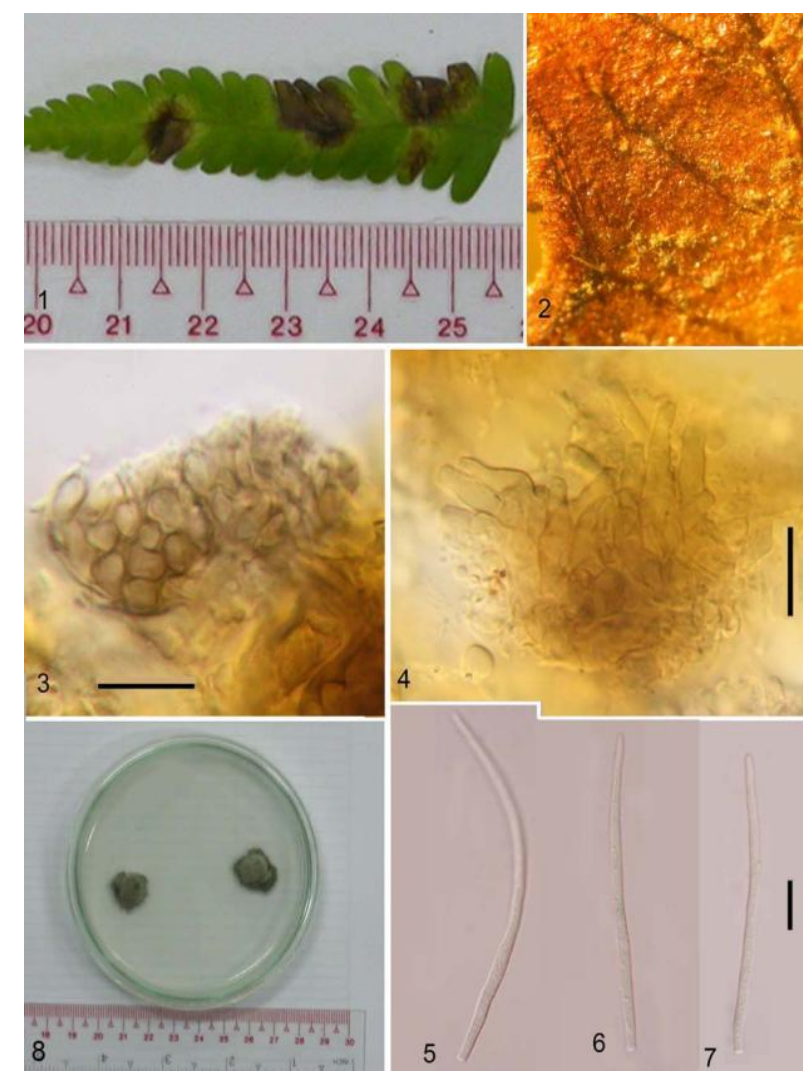

Fig. 61 - Pseudocercospora christellae on Christella parasitica from leaf spots: 1. Leaf spots on host leaf (upper surface). 2. Colonies. 3. Stroma. 4. Stroma with attached conidiophores. 5-7. Conidia. 8. Culture. Bars: 1, $8=$ $10 \mathrm{~mm} .3-7=10 \mu \mathrm{m}$.

\section{Pseudocercospora consociata (G. Winter)} Y.L. Guo \& X.J. Liu, Mycosystema 24: 232, 1989.

Figs 62-63.

$\equiv$ Cercospora consociate G. Winter, Hedwigia 22: 70, 1883.

Leaf spots subcircular to irregular, 1-7 mm diam., at first yellowish brown, and than dark brown in the centre, dark brown at the margin. Colonies amphigemous, conspicuous. Mycelium internal; hyphae branched, 3-5 $\mu \mathrm{m}$ wide $(\bar{x}=4.5 \mu \mathrm{m}, \mathrm{n}=5)$, septate, constricted at the septa, distance between septa 4-7 $\mu \mathrm{m}(\bar{x}$ $=5.6 \mu \mathrm{m}, \mathrm{n}=5$ ), brownish, subhyaline, wall $0.3-0.5 \mu \mathrm{m}$ wide $(\bar{x}=0.56 \mu \mathrm{m}, \mathrm{n}=5)$, smooth, forming plate-like plectenchymatous stromatic hyphal aggregations. Stromata oval to ellipsoidal, $20-55 \mu \mathrm{m}$ diam. $(\bar{x}=36.7 \mu \mathrm{m}, \mathrm{n}$ $=7$ ), brown to dark brown, stromatal cells oval, ellipsoidal to angular, $4-11 \mu \mathrm{m}$ wide $(\bar{x}=6$ $\mu \mathrm{m}, \mathrm{n}=30$ ), dark brown, wall $0.5-0.8 \mu \mathrm{m}$ wide $(\bar{x}=0.59 \mu \mathrm{m}, \mathrm{n}=30)$, smooth. Conidiophores fasciculate, arising from stromata (6-74 per fascicle), geniculate, unbranched, 9-27 $\times 2-4$ $\mu \mathrm{m}(\bar{x}=18.5 \times 3.13 \mu \mathrm{m}, \mathrm{n}=30), 0-1$-septate, slightly constricted at the septa, distance between septa 9-17 $\mu \mathrm{m}$ long $(\bar{x}=14.8 \mu \mathrm{m}, \mathrm{n}=$ 30 ), uniformly pale to medium brown, much paler and narrower towards the tip, wall 0.5 $0.8 \mu \mathrm{m}(\bar{x}=0.65 \mu \mathrm{m}, \mathrm{n}=30)$, smooth. Conidiogenous cells terminal, 9-17 $\times 2-3 \mu \mathrm{m}$ $(\bar{x}=14.8 \times 2.67 \mu \mathrm{m}, \mathrm{n}=11)$, apex obtuse, conidiogenous loci inconspicuous, unthickened, not darkened. Conidia solitary, obclavate, straight to slightly curved, 24-94 $\times$ $2-3 \mu \mathrm{m}(\bar{x}=56.8 \times 2.4 \mu \mathrm{m}, \mathrm{n}=13), 1-7-$ septate, pale olivaceous-brown, wall $0.3-0.5$ $\mu \mathrm{m}$ wide $(\bar{x}=0.38 \mu \mathrm{m}, \mathrm{n}=13)$, smooth, tip subacute, base obconically truncate, hila $0.72-$ $1.5 \mu \mathrm{m}$ wide $(\bar{x}=1.03 \mu \mathrm{m}, \mathrm{n}=13)$.

Known hosts - Dicliptera chinensis (L.) Juss., Dyschoriste oblongifolia (Michx.) Kuntze, Justicia gendarussa Burm. f., J. procumbens L., Ruellia sp. (Acanthaceae).

Known distribution - Asia: China, India, Japan, Thailand; North America and West Indies: USA (AL, FL, IA, IL, MS, OK); South America: Brazil, Venezuela.

Material examined - Chiang Rai Province, Muang District, Sri Pangsang Village, on leaves of Justicia gendarussa (Acanthaceae), 16 January 2010, P. Phengsintham (P528).

Notes - The collection from Thailand agrees well with the description of Pseudocercospora consociata in Chupp (1954) and Guo \& Hsieh (1995) except for relatively narrow conidia, which are, however, within the common range of conidia in this species. Guo \& Hsieh (1995) assigned a Chinese collection on Justicia procumbens to $P$. consociata, and Braun \& Urtiaga (2008) described $P$. consociata var. dimorpha U. Braun \& Urtiaga on Justicia galapagana from Venezuela, differing from typical $P$. consociata by the formation of well-developed superficial mycelium with solitary conidiophores. $P$. justiciae (F.L. Tai) Y.L. Guo \& X.J. Liu is quite distinct from $P$. consociata by its much longer and wider conidiophores $(50-130 \times 4$ $6.5 \mu \mathrm{m})$ and much wider conidia $40-110 \times$ 3.5-6.5 $\mu \mathrm{m}$.

Literature - Chupp (1954: 24); Guo \& Hsieh (1995: 2); Guo et al. (1998: 11); Crous \& Braun (2003: 234). 


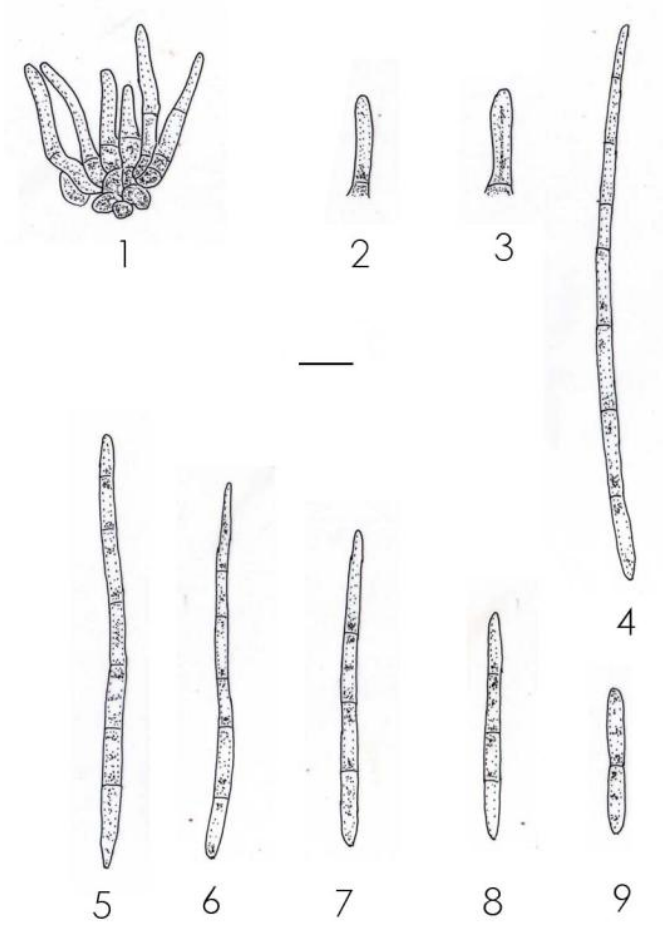

Fig. 62 - Pseudocercospora consociata on Justicia gendarussa from leaf spots: 1. Stroma with attached conidiophores. 2-3. Conidiophore. 5-9. Conidia. Bars: $1-9=10$ $\mu \mathrm{m}$.

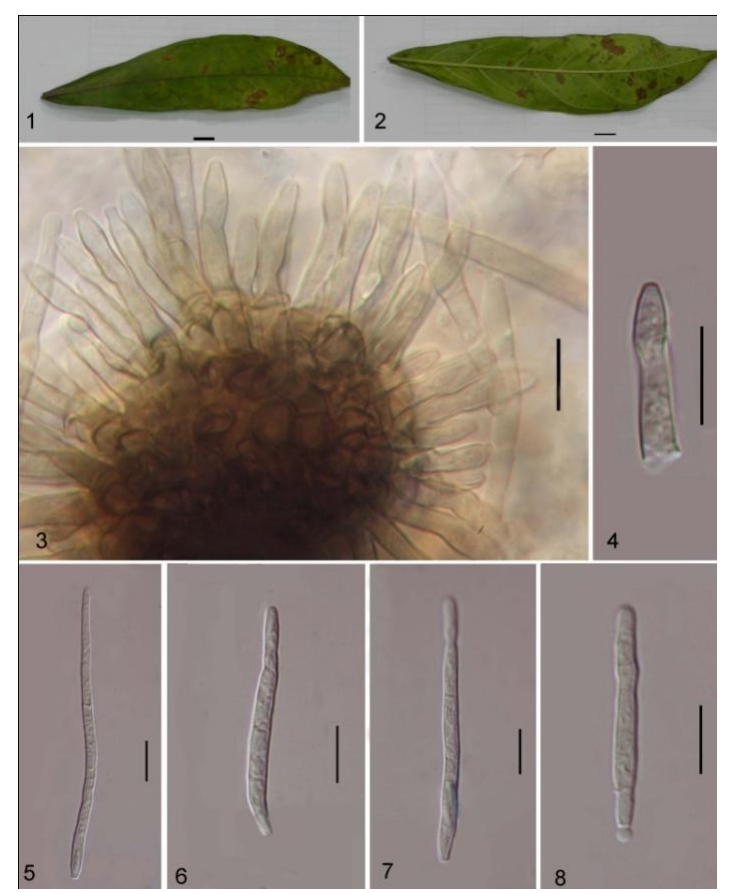

Fig. 63 - Pseudocercospora consociata on Justicia gendarussa from leaf spots: 1-2 Leaf spots on host leaves (1. upper surface, 2. lower surface). 3. Stroma with attached conidiophores. 4. Conidiophore. 5-8. Conidia. Bars: $1-2=10 \mathrm{~mm}, 3-8=10 \mu \mathrm{m}$.
Pseudocercospora cratevae sp. nov.

Figs 64-65.

Mycobank, MB 801348

Morphologically similar to Pseudocercospora conspicua, but distinct leaf spots formed, conidiophores very short and conidia pale olivaceous-brown.

Leaf spots subcircular to irregular, 1-13 $\mathrm{mm}$ diam., grey-brown in the center, brown to dark brown at the margin. Colonies amphigenous, inconspicuous. Mycelium internal, inconspicuous. Stromata oval to ellipsoidal, 9$12 \mu \mathrm{m}$ diam. $(\bar{x}=10.5 \mu \mathrm{m}, \mathrm{n}=5)$, brown to dark brown, stromatal cells oval, ellipsoidal and angular, 3-9 $\mu \mathrm{m}$ wide $(\bar{x}=5 \mu \mathrm{m}, \mathrm{n}=15)$, dark brown, wall $0.5-0.8 \mu \mathrm{m}$ wide $(\bar{x}=0.58$ $\mu \mathrm{m}, \mathrm{n}=15)$, smooth. Conidiophores solitary or fasciculate, arising from stromata (2-6 per fascicle), geniculate, unbranched, 9-16 × 2-4 $\mu \mathrm{m}(\bar{x}=12.35 \times 3.05 \mu \mathrm{m}, \mathrm{n}=15), 0-1-$ septate, slightly constricted at the septa, distance between septa 4-12 $\mu \mathrm{m}$ long $(\bar{x}=$ $7.64 \mu \mathrm{m}, \mathrm{n}=15$ ), uniformly pale to medium brown or much paler and more narrow toward the tip, wall $0.3-0.5 \mu \mathrm{m}(\bar{x}=0.47 \mu \mathrm{m}, \mathrm{n}=15)$, smooth. Conidiogenous cells terminal, 7-12 $\times$ $2-4 \mu \mathrm{m}(\bar{x}=9.64 \times 2.70 \mu \mathrm{m}, \mathrm{n}=14)$, apex obtuse, conidiogenous loci inconspicuous, unthickened, not darkened. Conidia solitary, obclavate, straight to slightly curved, 32-44 $\times$ $2-4 \mu \mathrm{m}(\bar{x}=38.52 \times 2.64 \mu \mathrm{m}, \mathrm{n}=13), 3-5-$ septate, pale olivaceous-brown, wall $0.3-0.5$ $\mu \mathrm{m}$ wide $(\bar{x}=0.36 \mu \mathrm{m}, \mathrm{n}=13)$, smooth, tip subacute, base obconically truncate, hila 1-2 $\mu \mathrm{m}$ wide $(\bar{x}=1.39 \mu \mathrm{m}, \mathrm{n}=13)$.

Known hosts - Crateva religiosa Forst.

f. (Capparaceae).

Known distribution - Asia: Thailand.

Material examined - Chiang Rai Province, Muang District, Sri Pangsang Village, on leaves of Crateva religiosa (Capparaceae), 22 August 2010, P. Phengsintham, P617 (MFLU12-2200, holotype).

Notes - This is the first record of a Pseudocercospora on this host. P. conspicua (Earle) Deighton, known from Australia, North America and the West Indies on Cleome spp. (Chupp 1954, Crous \& Braun 2003), is a morphologically similar species, which differs from $P$. cratevae in lacking leaf spots and in having longer conidiophores, up to $40 \mu \mathrm{m}$, and 
very pale, almost colourless conidia (Chupp 1954).

Pseudocercospora cycleae (Chidd.) Deighton, Mycol. Pap. 140: 143, $1976 . \quad$ Figs 66-67. Leaf spots subcircular to irregular, 1-10 mm diam., grey-brown in the center, brown to dark brown at the margin. Colonies amphigenous, conspicuous. Mycelium internal; hyphae branched, $2-5 \mu \mathrm{m}$ wide $(\bar{x}=4 \mu \mathrm{m}, \mathrm{n}=$ 7 ), septate, constricted at the septa, distance between septa $10-18 \mu \mathrm{m}(\bar{x}=15.71 \mu \mathrm{m}, \mathrm{n}=$ 7), subhyaline to brownish, wall $0.5-0.8 \mu \mathrm{m}$ wide $(\bar{x}=0.58 \mu \mathrm{m}, \mathrm{n}=7)$, smooth, forming plate-like plectenchymatous stromatic hyphal aggregations. Stromata oval to ellipsoidal, 20$40 \mu \mathrm{m}$ diam. $(\bar{x}=29.1 \mu \mathrm{m}, \mathrm{n}=10)$, brown to dark brown, stroma cells oval, ellipsoidal and angular, 5-10 $\mu \mathrm{m}$ wide $(\bar{x}=6.9 \mu \mathrm{m}, \mathrm{n}=30)$, dark brown, wall $0.5-1 \mu \mathrm{m}$ wide $(\bar{x}=0.61 \mu \mathrm{m}$, $\mathrm{n}=30$ ), smooth. Conidiophores fasciculate, arising from stromata (2-33 per fascicle), geniculate, unbranched, $37-143 \times 2-5 \mu \mathrm{m}(\bar{x}=$ $112 \times 3.8 \mu \mathrm{m}, \mathrm{n}=11), 2-7$-septate, slightly constricted at the septa, distance between septa 6-29 $\mu \mathrm{m}(\bar{x}=17.6 \mu \mathrm{m}, \mathrm{n}=30)$, uniformly pale to medium brown, paler and narrower towards the tip, wall $0.5-0.8 \mu \mathrm{m}$ wide $(\bar{x}=$ $0.62 \mu \mathrm{m}, \mathrm{n}=30$ ), smooth. Conidiogenous cells terminal, $6-28 \times 2-4 \mu \mathrm{m}(\bar{x}=17.1 \times 3 \mu \mathrm{m}, \mathrm{n}$ $=7$ ), apex obtuse; conidiogenous loci inconspicuous, unthickened, not darkened. Conidia solitary, obclavate, straight to slightly curved, $27-85 \times 4-6 \mu \mathrm{m}(\bar{x}=52.5 \times 4.6 \mu \mathrm{m}, \mathrm{n}=15)$, 2-10-septate, pale olivaceous-brown, wall $0.3-$ $0.5 \mu \mathrm{m}$ wide $(\bar{x}=0.39 \mu \mathrm{m}, \mathrm{n}=15)$, smooth, tip subacute, base obconically truncate, hila $0.72-2 \mu \mathrm{m}$ wide $(\bar{x}=1.52 \mu \mathrm{m}, \mathrm{n}=15)$.

Known hosts - Cyclea fissicalyx Dunn, C. peltata Hook. f. \& Thomson, Cyclea sp. (Menispermaceae).

Known distribution - Asia: China, India, Thailand.

Material examined - Chiang Rai Province, Khun Korn waterfall, on leaves of Cyclea peltata (Menispermaceae), 18 December 2009, P. Phengsintham (MFLU100319).

Notes - The collection from Khun Korn waterfall, Chiang Rai Province agrees with the

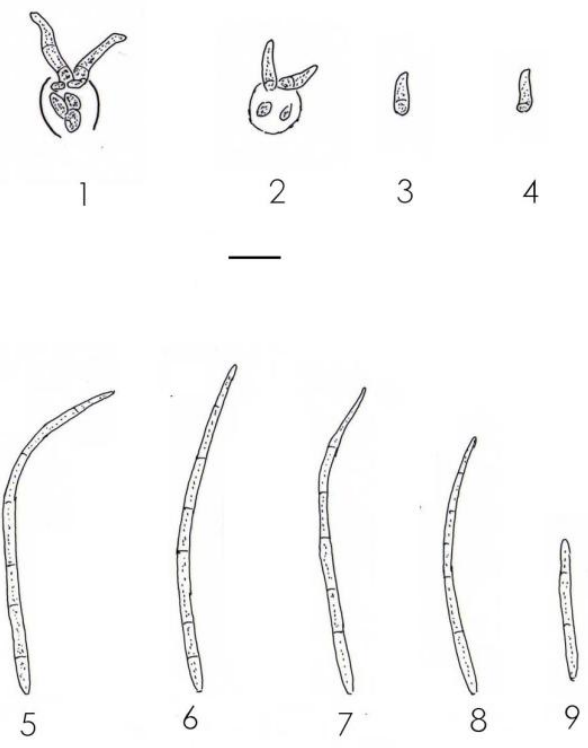

Fig. 64 - Pseudocercospora cratevae on Crateva religiosa from leaf spots: $1-2$. Stroma with attached conidiophores. 3-4. Conidiophores. 5-9. Conidia. Bar1: 1-9=10 $\mu \mathrm{m}$.

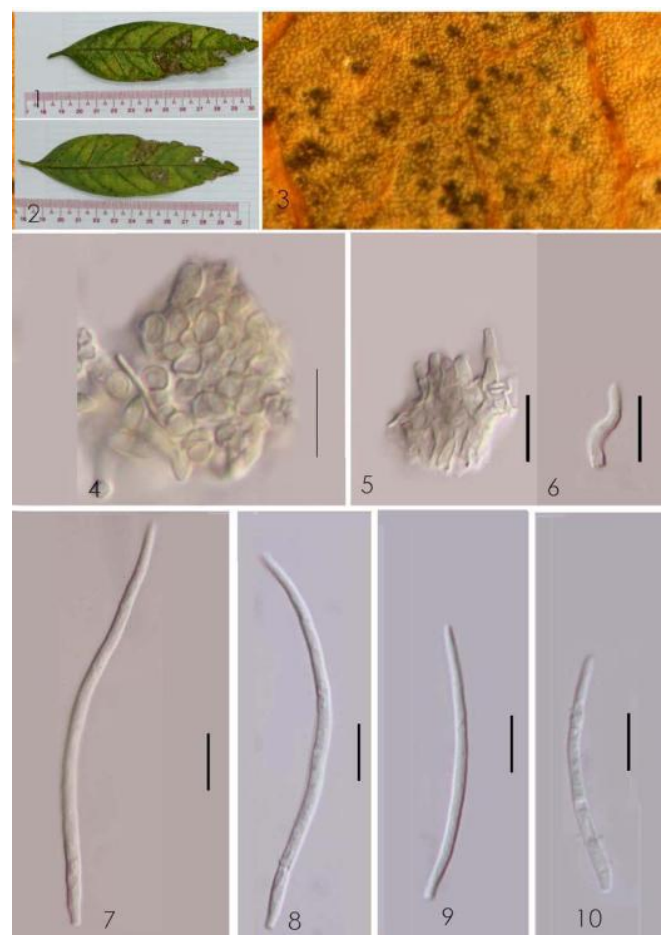

Fig. 65 - Pseudocercospora cratevae on Crateva religiosa from leaf spots: 1-2. Leaf spots on host leaves (1. upper surface, 2. lower surface). 3. Colonies. 4. Stroma. 5. Stroma with attached conidiophores. 6. Conidiophore. 7-10. Conidia. Bars: $1-2=10 \mathrm{~mm}$. 3. Not to scale. $4-10=10 \mu \mathrm{m}$. 

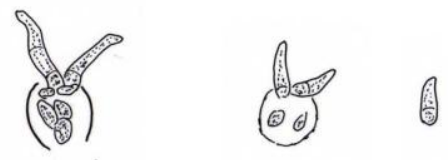

2

3

4

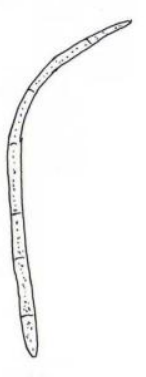

5
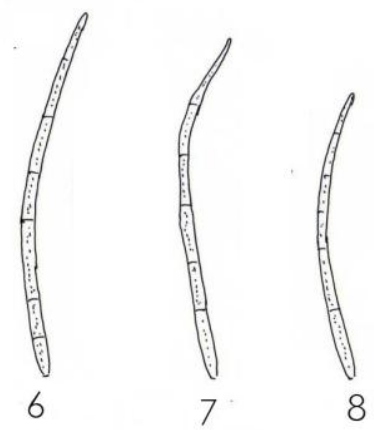

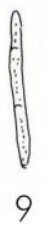

Fig. 66 - Pseudocercospora cycleae on Cyclea peltata from leaf spots: 1 . Stroma with attached conidiophores. 2-6. Conidia. Bars: $1-6=10 \mu \mathrm{m}$.

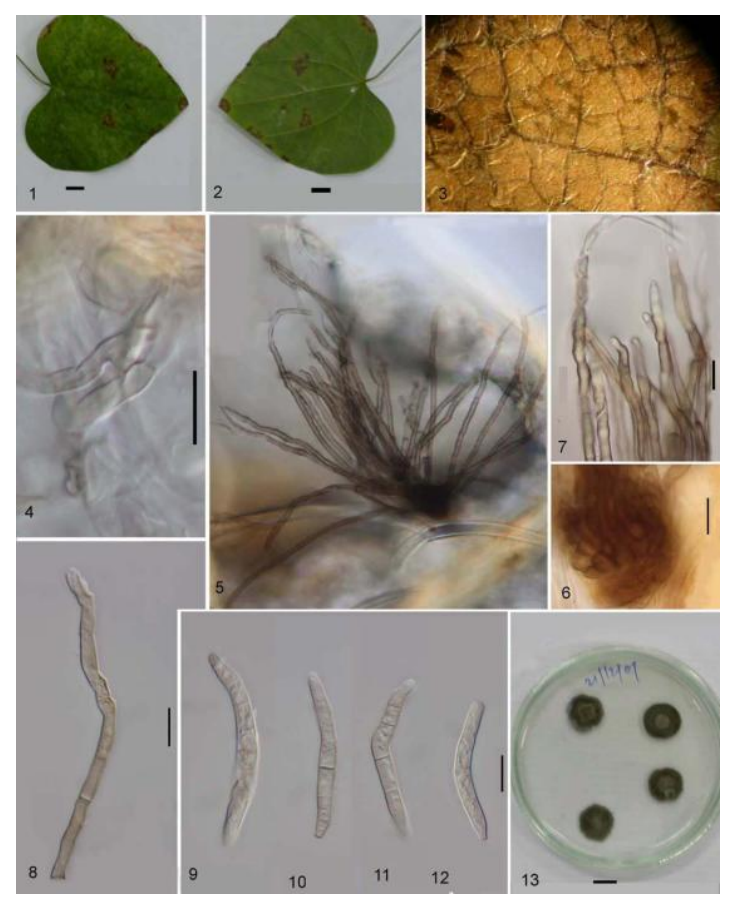

Fig. 67 - Pseudocercospora cycleae on Cyclea peltata from leaf spots: 1-2. Leaf spots on host leaves (1. upper surface, 2. lower surface). 3. Colonies. 4. Internal hyphae. 5. Stroma with attached conidiophores. 6. Stroma. 9. Apices of conidiophores. 8. Conidiophores. 9-12. Conidia. 13. Culture. Bars: $1-2=10 \mathrm{~mm} .3$. Not to scale. $4-12=10 \mu \mathrm{m} .13=10 \mathrm{~mm}$. description of this species in Guo \& Hsieh (1995), but differs in having longer conidiophores.

Literature - Guo \& Hsieh (1995: 203); Guo et al. (1998: 215); Crous \& Braun (2003: 149).

Pseudocercospora jahnii (Syd.) U. Braun \& Crous, in Crous and Braun, Mycosphaerella and its anamorphs: 1. Names published in Cercospora and Passalora. CBS Biodiversity Series 1: 230, 2003. Figs 68-69. 28: 214, 1930.

三 Cercospora jahnii Syd., Ann. Mycol.

Leaf spots subcircular to irregular, 1-5 mm diam., at first yellowish brown, and than dark brown in the centre, yellow at the margin. Colonies amphigenous, scattered. Mycelium internal; hyphae branched, 2-3 $\mu \mathrm{m}$ wide $(\bar{x}=$ $2.5 \mu \mathrm{m}, \mathrm{n}=6)$, septate, constricted at the septa, distance between septa 6-12 $\mu \mathrm{m}(\bar{x}=9 \mu \mathrm{m}, \mathrm{n}$ $=6$ ), brownish, subhyaline, wall $0.3-0.5 \mu \mathrm{m}$ wide ( $\bar{x}=0.4 \mu \mathrm{m}, \mathrm{n}=6$ ), smooth, forming plate-like plectenchymatous stromatic hyphal aggregations. Stromata oval to ellipsoidal, 20$34 \mu \mathrm{m}$ diam. ( $\bar{x}=27.60 \mu \mathrm{m}, \mathrm{n}=4)$, brown to dark brown, stromatal cells oval, ellipsoidal to angular, $2-5 \mu \mathrm{m}$ wide $(\bar{x}=3.38 \mu \mathrm{m}, \mathrm{n}=10)$, dark brown, wall $0.3-0.5 \mu \mathrm{m}$ wide $(\bar{x}=0.45$ $\mu \mathrm{m}, \quad \mathrm{n}=10)$, smooth. Conidiophores fasciculate, arising from stromata (8-17 per fascicle), not geniculate, unbranched, 6-10 $\times$ 2-4 $\mu \mathrm{m}(\bar{x}=8.38 \times 3.25 \mu \mathrm{m}, \mathrm{n}=9), 0-1-$ septate, slightly constricted at the septa, distance between septa 4-6 $\mu \mathrm{m}$ long $(\bar{x}=4.60$ $\mu \mathrm{m}, \mathrm{n}=9$ ), uniformly pale to medium brown or much paler and narrower towards the tip, wall $0.3-0.5 \mu \mathrm{m}(\bar{x}=0.48 \mu \mathrm{m}, \mathrm{n}=9)$, smooth. Conidiogenous cells terminal, 5-9 $\times 2-4 \mu \mathrm{m}$ $(\bar{x}=6.11 \times 2.70 \mu \mathrm{m}, \mathrm{n}=9)$, apex obtuse, conidiogenous loci inconspicuous, neither thickened nor darkened. Conidia solitary, obclavate, straight to slightly curved, 42-64 $\times$ $2-3 \mu \mathrm{m}(\bar{x}=52.41 \times 2.73 \mu \mathrm{m}, \mathrm{n}=13), 4-8-$ septate, pale olivaceous-brown, wall $0.3-0.5$ $\mu \mathrm{m}$ wide $(\bar{x}=0.36 \mu \mathrm{m}, \mathrm{n}=13)$, smooth, tip subacute, base obconically truncate, hila 1-2 $\mu \mathrm{m}$ wide $(\bar{x}=1.24 \mu \mathrm{m}, \mathrm{n}=13)$.

Known hosts - Tabebuia argentea (Bureau \& K. Schum.) Britton, T. chrysotricha (Mart. ex A. DC.) Standl., T. heterophylla (DC.) Britton, T. pentaphylla (L.) Hemsl., T. 
rosea (Bertol.) A. DC., T. serratifolia (Vahl) G. Nicholson, T. shaferi Britton (Bignoniaceae).

Known distribution - Africa: Senegal; Asia: India, Thailand; North America and West Indies: Cuba, Panama, Trinidad and Tobago, USA (FL); Oceania: Puerto Rico, Virgins Islands; South America: Brazil, Venezuela.

Material examined - Chiang Rai Province, Muang District, Sri Pangsang Village, on leaves of Tabebuia chrysotricha (Bignoniaceae), 4 August 2010, P. Phengsintham (P613). GenBank accession no (ITS, KC677903; LSU, KC677933).

Notes - The collection from Thailand differs from the description of $P$. jahnii by Chupp (1954) [conidiophores 10-40 × 3-5 $\mu \mathrm{m}$ and conidia 30-80 $\times 4-5.5 \mu \mathrm{m}]$ in having shorter conidiophores.

Literature - Chupp (1954: 86); Crous \& Braun (2003: 230).

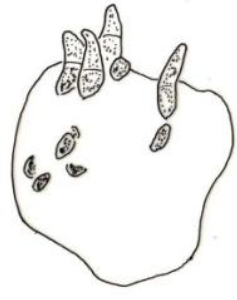

1

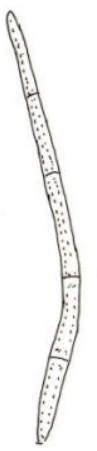

3

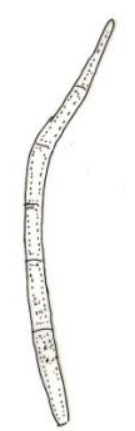

2

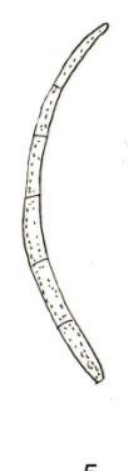

5
Fig. 68 - Pseudocercospora jahnii on Tabebuia chrysotricha: 1. Stroma with attached conidiophores. 2-5. Conidia. Bars: $1-5=10$ $\mu \mathrm{m}$.

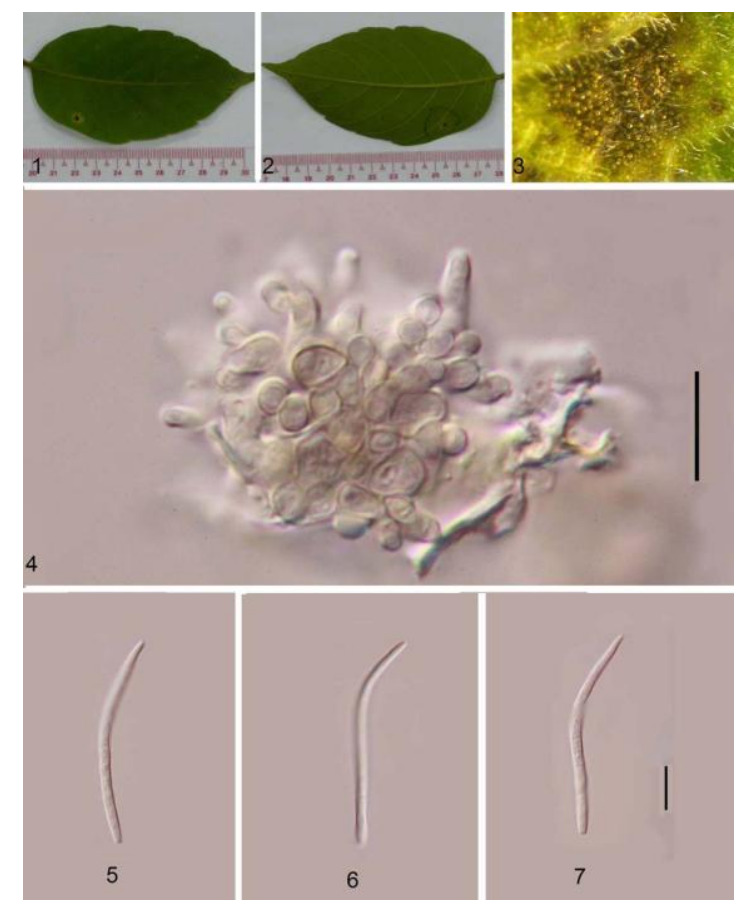

Fig. 69 - Pseudocercospora jahnii on Tabebuia chrysotricha from leaf spots: 1-2. Leaf spots on host leaves (1. upper surface, 2. lower surface). 3. Colonies. 4. Stroma with attached conidiophores. 5-7. Conidia. Bars: 1$2=10 \mathrm{~mm}$. 3. Not to scale. $4-7=10 \mu \mathrm{m}$.

Pseudocercospora lygodii Goh \& W. H. Hsieh, Trans. Mycol. Soc. Republ. China 2: 131, 1987.

Figs 70-71.

= Cercospora lygodii Sawada, Rep. Gov. Agric. Res. Inst. Taiwan 87: 83, 1944.

Leaf spots subcircular to irregular, 3-7 mm diam., dark brown in the centre, brown and dark brown at the margin. Colonies amphigenous, conspicuous. Mycelium internal, inconspicuous. Stromata oval to ellipsoidal, 10$60 \mu \mathrm{m}$ diam. $(\bar{x}=39.6 \mu \mathrm{m}, \mathrm{n}=5)$, brown to dark brown, stromatal cells oval, ellipsoidal to angular, $4-11 \mu \mathrm{m}$ wide $(\bar{x}=6.7 \mu \mathrm{m}, \mathrm{n}=15)$, dark brown, wall $0.5-0.8 \mu \mathrm{m}$ wide $(\bar{x}=0.53$ $\mu \mathrm{m}, \quad \mathrm{n}=15), \quad$ smooth. Conidiophores fasciculate, arising from stromata (4-30 per fascicle), geniculate, unbranched, 13-46 $\times 2.5-$ $4 \mu \mathrm{m}(\bar{x}=29.4 \times 3.50 \mu \mathrm{m}, \mathrm{n}=13), 0-4-$ septate, slightly constricted at the septa, distance between septa 4-30 $\mu \mathrm{m}$ long ( $\bar{x}=$ $12.3 \mu \mathrm{m}, \mathrm{n}=20$ ), uniformly pale to medium brown, much paler and narrower towards the tip, wall approximately $0.5-0.8 \mu \mathrm{m}(\bar{x}=0.61$ $\mu \mathrm{m}, \mathrm{n}=20$ ), smooth. Conidiogenous cells terminal, $12-30 \times 2-4 \mu \mathrm{m}(\bar{x}=16 \times 2.72 \mu \mathrm{m}$, $\mathrm{n}=9$ ), apex obtuse, conidiogenous loci 
inconspicuous, unthickened, not darkened. Conidia solitary, obclavate, straight to slightly curved, 36-120 × 2-4 $\mu \mathrm{m}(\bar{x}=80.4 \times 3 \mu \mathrm{m}, \mathrm{n}$ $=15)$, 4-11-septate, pale olivaceous-brown, wall $0.3-0.5 \mu \mathrm{m}$ wide $(\bar{x}=0.46 \mu \mathrm{m}, \mathrm{n}=15)$, smooth, tip subacute, base obconically truncate, hila $1.15-2 \mu \mathrm{m}$ wide $(\bar{x}=1.83 \mu \mathrm{m}, \mathrm{n}$ $=15)$.

Known hosts - Lygodium flexuosum (L.) Sw., Lygodium japonicum (Thunb.) Sw., L. macrostachyum Tagawa (Schizaeaceae).

Known distribution - Asia: Taiwan, Thailand.

Material examined - Chiang Mai Province, Maeteng District, Phadeng Village, on leaves of Lygodium flexuosum (Schizaeaceae), 7 June 2011, P. Phengsintham (P625).

Notes - The collection from Thailand agrees with the description of Pseudocercospora lygodii published by Chupp (1954) and Hsieh \& Goh (1990) [conidiophores 20-90 $\times 3-4 \mu \mathrm{m}$ and conidia $10-35 \times 1.5-3 \mu \mathrm{m}]$.

Literature - Chupp (1954: 456); Hsieh \& Goh (1990: 305); Guo \& Hsieh (1995: 187); Guo et al. (1998: 199); Crous \& Braun (2003: 258).
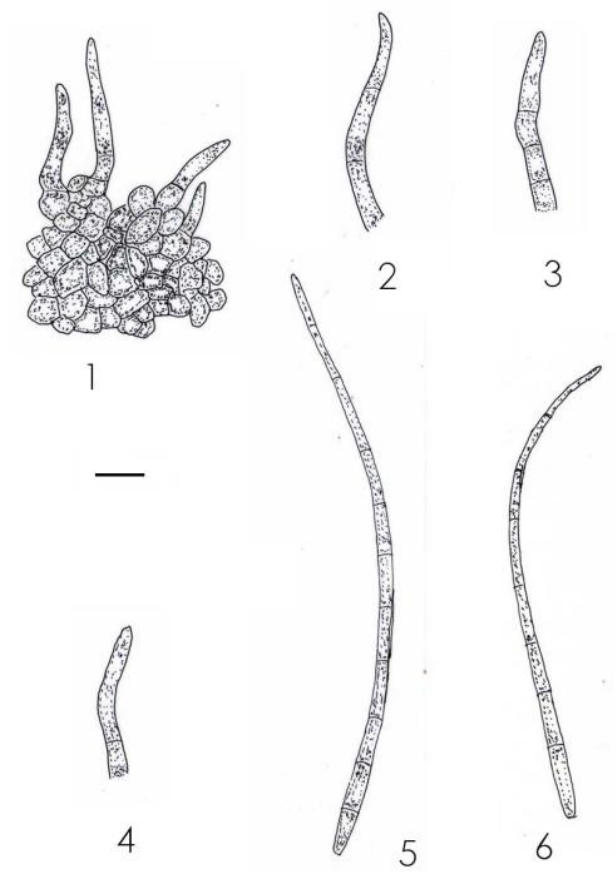

Fig. 70 - Pseudocercospora lygodii on Lygodium flexuosum from leaf spots: 1. Stroma with attached conidiophores. 2-4. Conidiophores. 5-6. Conidia. Bars: $1-6=10$ $\mu \mathrm{m}$.

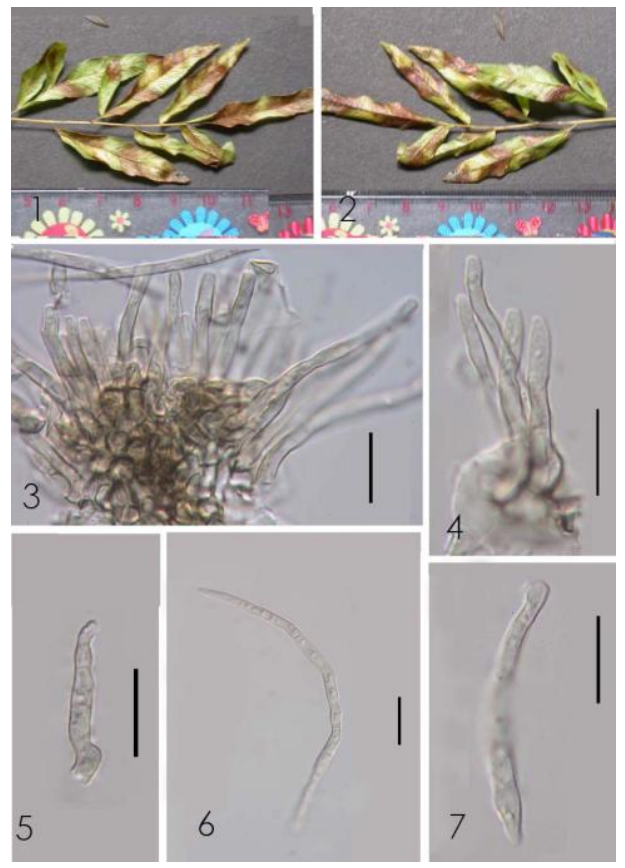

Fig. 71 - Pseudocercospora lygodii on Lygodium flexuosum from leaf spots: 1-2. Leaf spots on host leaves (1. upper surface, 2. lower surface). 3-4. Stromata with attached conidiophores. 5. Conidiophore. 6-7. Conidia. Bars: $1-2=10 \mathrm{~mm}, 3-7=10 \mu \mathrm{m}$.

Pseudocercospora malloticola Goh \& W.H. Hsieh, Cercospora and similar fungi from Taiwan: 124, 1990 Figs 72-73. = Cercospora malloti Ellis \& Everh. sensu Sawada, Taiwan Agric. Res. Inst. Rept 85: 113 (1943a); also 86: 173 (1943b); non Cercospora malloti Ellis \& Everh. (J. Mycol. 4: 114, 1888).

Leaf spots circular to irregular, 1-6 mm diam., at first yellowish, later becoming brown or dark brown, and with yellowish margin. Colonies amphigenous, inconspicious. Stromata well-developed, substomatal, oval, ellipsoidal, $10-40 \mu \mathrm{m}$ wide $(\bar{x}=29.9 \mu \mathrm{m}, \mathrm{n}=11)$, brown to dark brown, stomatal cells oval, angular to obclavate in outline, 3-7 $\mu \mathrm{m}$ wide $(\bar{x}=5.2 \mu \mathrm{m}, \mathrm{n}=28)$, wall $0.5-0.8 \mu \mathrm{m}$ wide $(\bar{x}$ $=0.52 \mu \mathrm{m}, \mathrm{n}=28)$, smooth. Conidiophores fasciculate, arising from stromata (2-11 per fascicle), emerging through stomata, nearly straight or cylindrical, simple, unbranched, 10$40 \times 3-5 \mu \mathrm{m}(\bar{x}=20.3 \times 4.1 \mu \mathrm{m}, \mathrm{n}=10), 0-2-$ septate, distance between septa 10-20 $\mu \mathrm{m}(\bar{x}=$ $13.7 \mu \mathrm{m}, \mathrm{n}=17$ ), medium brown, paler at the apex, uniformly pale to medium brown, or much paler and more narrower toward the tip, 
thin-walled $0.5-0.6 \mu \mathrm{m}(\bar{x}=5.2 \mu \mathrm{m}, \mathrm{n}=17)$, smooth. Conidiogenous cells terminal, 10-20 $\times$ 3-5 $\mu \mathrm{m}(\bar{x}=15 \times 3.9 \mu \mathrm{m}, \mathrm{n}=10)$, nearly straight or cylindrical; walled $0.5-0.6 \mu \mathrm{m}(\bar{x}=$ $5.1 \mu \mathrm{m}, \mathrm{n}=11)$; conidiogenous loci inconspicuous or subdenticulate, unthickened, not darkened. Conidia formed singly, obclavatecylindrical, straight to slightly curved, 33-75 $\times$ 3-4 $\mu \mathrm{m}(\bar{x}=58.5 \times 3.6 \mu \mathrm{m}, \mathrm{n}=13), 3-7-$ septate, pale olivaceous-brown, wall $0.3-0.5$ $\mu \mathrm{m}(\bar{x}=0.33 \mu \mathrm{m}, \mathrm{n}=13)$, smooth, apex subacute, base obconically truncate, hila $1.5-2$ $\mu \mathrm{m}$ wide $(\bar{x}=1.8 \mu \mathrm{m}, \mathrm{n}=10)$, wall $0.3-0.5$ $\mu \mathrm{m}$ wide $(\bar{x}=0.44 \mu \mathrm{m}, \mathrm{n}=10)$, unthickened and not darkened.

Known hosts - Mallotus barbatus (Wall.) Müll. Arg., M. japonicus (L. f.) Müll. Arg., M. thorelii Gagnep. (Euphorbiaceae).

Known distribution - Asia: Laos, Taiwan, Thailand.

Material examined - Chiang Rai Province, Muang District, Sri Pangsang Village, on leaves of Mallotus barbatus (Euphorbiaceae), 30 August 2009, P. Phengsintham (MFLU10-0320). GenBank accession no (ITS, KC677906).

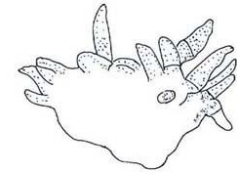

1

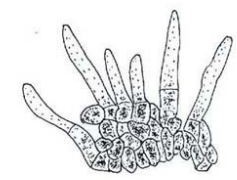

2

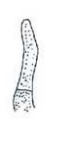

3
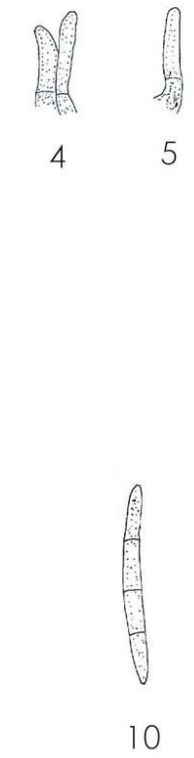

Fig. 72 - Pseudocercospora malloticola on Mallotus barbatus: 1-2. Stromata with attached conidiophores. 3-5. Conidiophores. 6-10. Conidia. Bars: $1-10=10 \mu \mathrm{m}$.

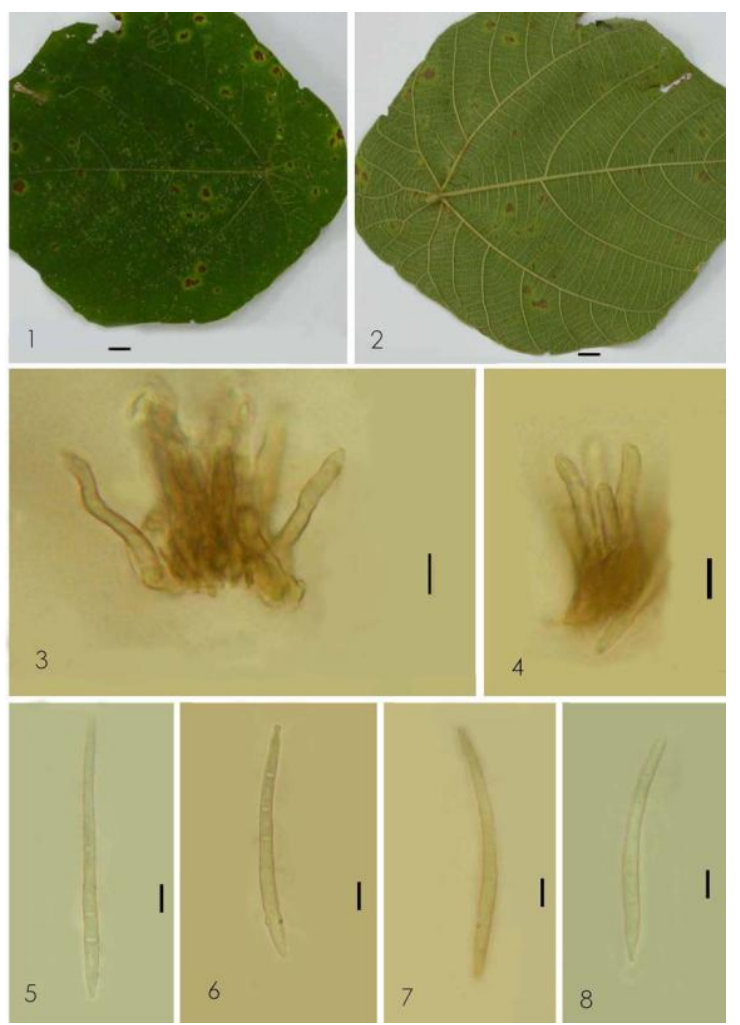

Fig. 73 - Pseudocercospora malloticola on Mallotus barbatus on leaf spots: 1-2. Leaf spots (1. upper surface, 2. lower surface). 3-4. Stromata with attached conidiophores. 5-8. Conidia. Bars: $1-2=10 \mathrm{~mm}, 3-8=10 \mu \mathrm{m}$.

Notes - The collection MFLU10-0320 from Sri Pangsang Village, Chiang Rai Province is similar to $P$. malloticola from Taiwan described by Hsieh \& Goh (1990).

Literature - Hsieh \& Goh (1990: 124).

Pseudocercospora mombin (Petr. \& Cif.) Deighton, Mycol. Pap. 140: 148, 1976.

Figs 74-75.

= Cercospora mombin Petr. \& Cif., Ann. Mycol. 30: 322, 1932.

Leaf spots irregular, 5-40 $\mathrm{mm}$ diam., grey-brown or brown in the center, and with dark brown margin. Colonies amphigenous, conspicuous, scattered. Mycelium internal, sparsely developed; hyphae small, branched, intercellular, $2-4 \mu \mathrm{m}$ wide $(\bar{x}=2.75 \mu \mathrm{m}, \mathrm{n}=$ $15)$, septate, constricted at the septa, distance between septa $4-8 \mu \mathrm{m}(\bar{x}=6.25 \mu \mathrm{m}, \mathrm{n}=15)$, hyaline to subhyaline, thin-walled $0.5-0.8 \mu \mathrm{m}$ wide $(\bar{x}=0.57 \mu \mathrm{m}, \mathrm{n}=15)$, smooth, forming plate-like plectenchymatous structures; external hyphae lacking. Stromata well-developed, substomatal, oval, ellipsoidal, $18-53 \mu \mathrm{m}$ wide 
$(\bar{x}=32 \mu \mathrm{m}, \mathrm{n}=5$ ), brown to dark brown, stomatal cells oval, angular to obclavate in outline, $3-7 \mu \mathrm{m}$ wide $(\bar{x}=4.7 \mu \mathrm{m}, \mathrm{n}=30)$, wall $0.5-1 \mu \mathrm{m}$ wide $(\bar{x}=0.66 \mu \mathrm{m}, \mathrm{n}=30)$, smooth. Conidiophores fasciculate, arising from stromata (2-18 per fascicle), emerging through stomata, nearly straight or cylindrical, simple, unbranched, not geniculate, 4-24 $\times$ $1.5-4 \mu \mathrm{m}(\bar{x}=12.3 \times 2.9 \mu \mathrm{m}, \mathrm{n}=15), 0-1-$ septate, distance between septa 4-24 $\mu \mathrm{m}(\bar{x}=$ $9.9 \mu \mathrm{m}, \mathrm{n}=15)$, medium brown, paler at the apex, uniformly pale to medium brown, or much paler and more narrower toward the tip, thin-walled $0.3-0.5 \mu \mathrm{m}(\bar{x}=0.36 \mu \mathrm{m}, \mathrm{n}=15)$, smooth. Conidiogenous cells terminal, 4-24 $\times$ $1.5-4 \mu \mathrm{m}(\bar{x}=10.6 \times 2.88 \mu \mathrm{m}, \mathrm{n}=10)$, nearly straight or cylindrical; walled $0.5-0.8 \mu \mathrm{m}(\bar{x}=$ $0.38 \mu \mathrm{m}, \mathrm{n}=10$ ); conidiogenous loci inconspicuous or subdenticulate, unthickened, not darkened. Conidia formed singly, cylindrical, straight to slightly curved, $21-57 \times 1-2 \mu \mathrm{m}(\bar{x}$ $=37.7 \times 1.55 \mu \mathrm{m}, \mathrm{n}=15), 2-7$ - septate, pale olivaceous-brown, wall $0.3-0.5 \mu \mathrm{m}(\bar{x}=0.32$ $\mu \mathrm{m}, \mathrm{n}=15$ ) wide, smooth, apex subacute, base obconically truncate, hila $0.5-1.5 \mu \mathrm{m}$ wide $(\bar{x}$ $=0.7 \mu \mathrm{m}, \mathrm{n}=15)$, wall $0.3-0.5 \mu \mathrm{m}$ wide $(\bar{x}=$ $0.32 \mu \mathrm{m}, \mathrm{n}=15)$, thickened and darkened.

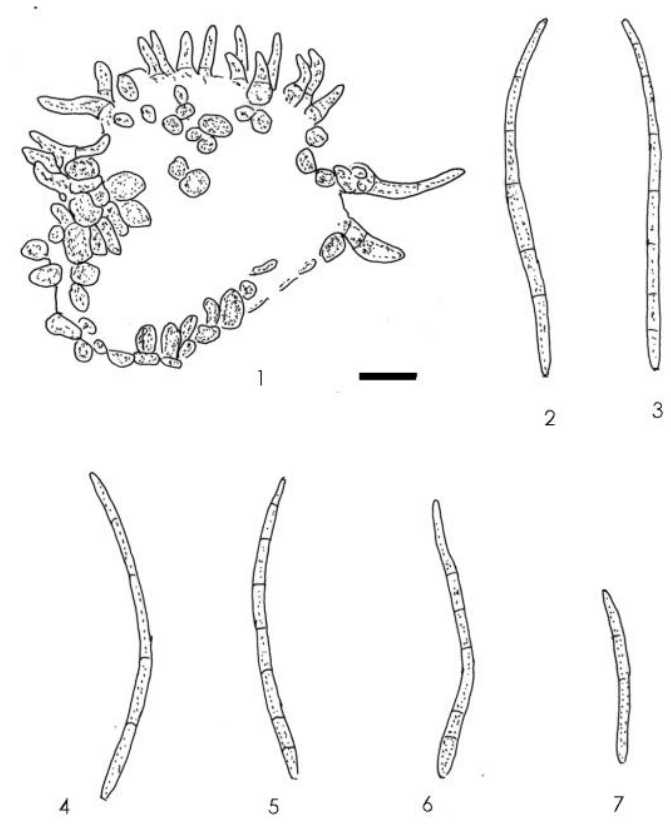

Fig. 74 - Pseudocercospora mombin on Spondias pinnata on leaf spots: 1 . Stroma with attached conidiophores. 2-7. Conidia. Bars: $1-7=10 \mu \mathrm{m}$.

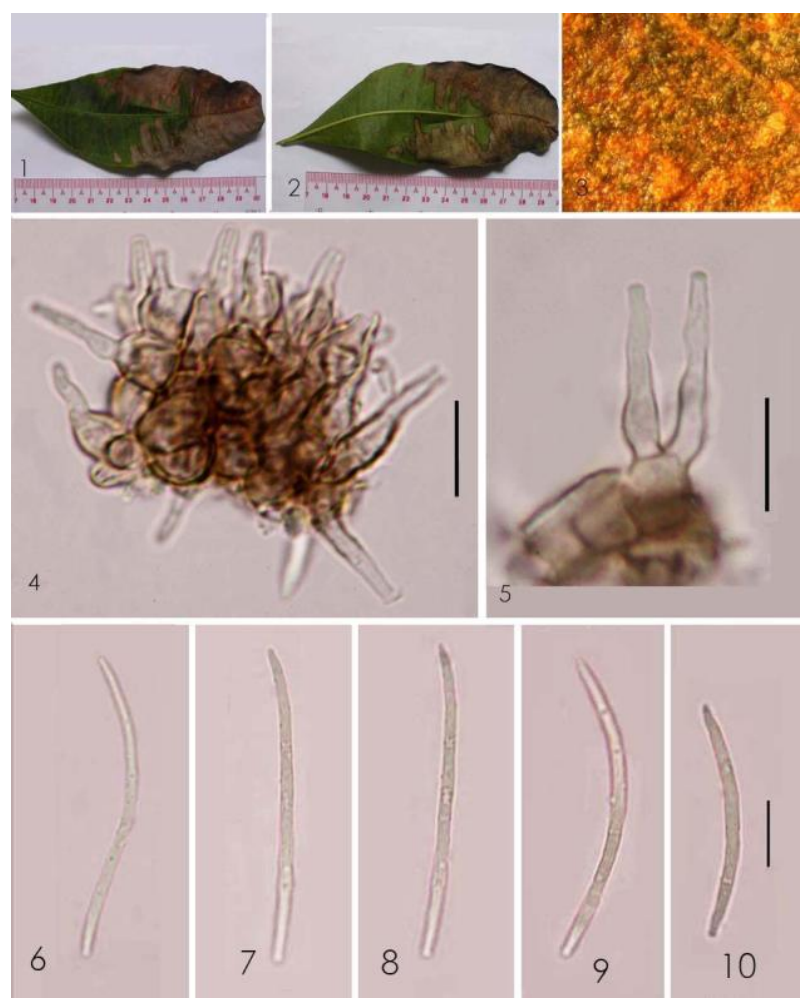

Fig. 75 - Pseudocercospora mombin on Spondias pinnata on leaf spots: 1-2. Leaf spots on host leaves (1. upper surface, 2. lower surface). 3. Colonies. 4-5. Stromata with attached conidiophores. 6-10. Conidia. Bars: $1-2=10 \mathrm{~mm}, 3$. Not to scale. $4-10=10 \mu \mathrm{m}$.

Known hosts - Spondias mombin L., S. pinnata (L.F.) Kurz, S. purpurea L. (Anacardiaceae).

Known distribution - Asia: Philippines, Thailand; North America and West Indies: Cuba, Dominican Rep., Panama; South America: Brazil, Venezuela.

Material examined - Chiang Rai Province, Muang District, Ban Dou Village, on leaves of Spondias pinnata (Anacardiaceae), 24 August 2010, P. Phengsintham (P625). GenBank accession no (ITS, KC677907; LSU, KC677935).

Notes - The collection from Ban Dou Village, Chiang Rai Province (Thailand) is similar to $P$. mombin (= Cercospora mombin) from the Dominican Republic described by Chupp (1954) [conidiophores 5-30 × 3-4 $\mu \mathrm{m}$ and conidia 20-85 $\times 2-3.5 \mu \mathrm{m}]$.

Literature - Chupp (1954: 41); Crous \& Braun (2003: 279). 
Pseudocercospora mori (Hara) Deighton, Mycol. Pap. 140: 148, 1976. Figs 76-77. $\equiv$ Cercospora mori Hara, J. Sericult. Assoc. Japan 27: 227, 1918.

$=$ Cercospora mori Marshall \& Steyaert, Bull. Soc. Roy. Bot. Belgique 61: 166, 1929.

Leaf spots irregular, 1-5 $\mathrm{mm}$ diam., brown or brown in the center, and with yellowish margin. Colonies amphigenous, conspicuous, scattered. Mycelium internal, inconspicuous. Stromata developed, substomatal, oval, ellipsoidal, $13-50 \mu \mathrm{m}$ wide $(\bar{x}=31.5$ $\mu \mathrm{m}, \mathrm{n}=5$ ), brown to dark brown, stomatal cells oval, angular to obclavate in outline, 4-7 $\mu \mathrm{m}$ wide $(\bar{x}=6 \mu \mathrm{m}, \mathrm{n}=30)$, wall $0.5-0.8 \mu \mathrm{m}$ wide $(\bar{x}=0.62 \mu \mathrm{m}, \mathrm{n}=30)$, smooth. Conidiophores fasciculate, arising from stromata (2-18 per fascicle), emerging through stomata, nearly straight or cylindrical, simple, unbranched, $8-17 \times 3-5 \mu \mathrm{m}(\bar{x}=12.5 \times 4 \mu \mathrm{m}$, $\mathrm{n}=9$ ), 0-1-septate, distance between septa 6-8 $\mu \mathrm{m}(\bar{x}=7 \mu \mathrm{m}, \mathrm{n}=10)$, medium brown, paler at the apex, uniformly pale to medium brown, or much paler and more narrower toward the tip, thin-walled $0.5-0.8 \mu \mathrm{m}(\bar{x}=0.6 \mu \mathrm{m}, \mathrm{n}=$ 9), smooth. Conidiogenous cells terminal, 7-8 $\times 3-4 \mu \mathrm{m}(\bar{x}=7.5 \times 3.5 \mu \mathrm{m}, \mathrm{n}=5)$, nearly straight or cylindrical; wall $0.5-0.8 \mu \mathrm{m}(\bar{x}=$ $0.65 \mu \mathrm{m}, \mathrm{n}=5$ ); conidiogenous loci inconspicuous or subdenticulate, unthickened, not darkened. Conidia formed singly, cylindrical, straight to slightly curved, 27-46 $\times$ 2.5-4 $\mu \mathrm{m}(\bar{x}=33.2 \times 3.12 \mu \mathrm{m}, \mathrm{n}=15), 2-7-$ septate, pale olivaceous-brown, wall $0.3-0.5$ $\mu \mathrm{m}(\bar{x}=0.35 \mu \mathrm{m}, \mathrm{n}=15)$, smooth, apex subacute, base obconically truncate, hila 1-2 $\mu \mathrm{m}$ wide $(\bar{x}=1.37 \mu \mathrm{m}, \mathrm{n}=15)$, wall $0.3-0.5$ $\mu \mathrm{m}$ wide $(\bar{x}=0.45 \mu \mathrm{m}, \mathrm{n}=15)$, unthickened and not darkened.

Known hosts - Morus acidosa Griff., M. alba L., M. australis Poir., M. indica L., $M$. nigra L., M. rubra L. (Moraceae).

Known distribution - Africa: Congo;

Asia: Bangladesh, China, India, Japan, Lebanon, Myanmar, Pakistan, Singapore, Taiwan, Thailand; Europe: Belgium, Georgia; North America and West Indies: Cuba, Dominican Rep. Panama; USA (AL).

Material examined - Chiang Rai
Province, Maechan District, Doitung National Park, on leaves of Morus alba (Moraceae), 12 April 2009, P. Phengsintham (P484). GenBank accession no (ITS, KC731557).

Notes - The collection from Doitung National Park, Chiang Rai Province (Thailand) is similar to P. mori described by Hsieh \& Goh (1990) [conidiophores 20-90 × 3-5 $\mu \mathrm{m}$ and conidia 20-80 × 3-5 $\mu \mathrm{m}$ ].

Literature - Chupp (1954: 399); Saccardo (1972: 1378, 1384); Yen \& Lim (1980: 180); Hsieh \& Goh (1990: 240); Guo \& Hsieh (1995: 217); Crous \& Braun (2003: 282).

Pseudocercospora olacicola (Muthappa) Kamal, M.K. Khan \& R.K. Verma, Mycol. Res. 94: 241, 1990.

Figs 78-79.

$\equiv$ Cercospora olacicola Muthappa, Sydowia 21: 156, (1967)1968.

Leaf spots subcircular to irregular, 1-5 $\mathrm{mm}$ diam., at first yellowish, later becoming brown in the center, brown to yellowish brown at the margin. Colonies amphigenous, scattered, conspicuous. Mycelium internal, inconspicuous. Stromata oval to ellipsoidal, 15-32 $\mu \mathrm{m}$ diam. $(\bar{x}=22.3 \mu \mathrm{m}, \mathrm{n}=7)$, brown to dark brown, stromatal cells oval, ellipsoidal 15-32 $\mu \mathrm{m}$ diam. $(\bar{x}=22.3 \mu \mathrm{m}, \mathrm{n}=7)$, brown to dark brown, stromatal cells oval, ellipsoidal and angular, $3-7 \mu \mathrm{m}$ wide $(\bar{x}=5.3 \mu \mathrm{m}, \mathrm{n}=$ 15), dark brown, wall $0.5-0.8 \mu \mathrm{m}$ wide $(\bar{x}=$ $0.58 \mu \mathrm{m}, \mathrm{n}=15$ ), smooth. Conidiophores fasciculate, arising from stromata (3-12 per fascicle), geniculate, unbranched, 13-34 × 3-4 $\mu \mathrm{m}(\bar{x}=23.3 \times 3.56 \mu \mathrm{m}, \mathrm{n}=19), 0-3$-septate, simple or branched, slightly constricted at the septa, distance between septa 4-20 $\mu \mathrm{m}$ long $(\bar{x}$ $=10.4 \mu \mathrm{m}, \mathrm{n}=30$ ), uniformly pale to medium brown or much paler and more narrow toward the tip, wall $0.5-0.8 \mu \mathrm{m}(\bar{x}=0.56 \mu \mathrm{m}, \mathrm{n}=30)$, smooth. Conidiogenous cells terminal, 9-20 $\times$ $2-4 \mu \mathrm{m}(\bar{x}=13.1 \times 3 \mu \mathrm{m}, \mathrm{n}=9)$, apex obtuse; conidiogenous loci inconspicuous, unthickened, not darkened. Conidia solitary, obclavate, straight to slightly curved, $47-83 \times$ 3-4 $\mu \mathrm{m}(\bar{x}=62.2 \times 3.2 \mu \mathrm{m}, \mathrm{n}=15), 4-6-$ septate, pale olivaceous-brown, wall $0.5-0.8$ $\mu \mathrm{m}$ wide $(\bar{x}=0.56 \mu \mathrm{m}, \mathrm{n}=15)$, smooth, tip subacute, base obconically truncate, hila $0.7-2$ $\mu \mathrm{m}$ wide $(\bar{x}=1.23 \mu \mathrm{m}, \mathrm{n}=15)$. 

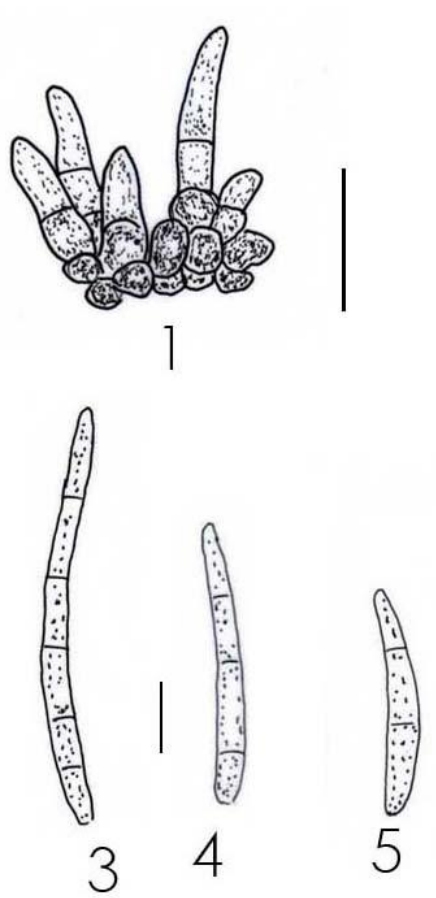

Fig. 76 - Pseudocercospora mori on Molus alba from leaf spots: 1. Stroma with attached conidiophores. 2-6. Conidia. Bars: $1-6=10 \mu \mathrm{m}$.

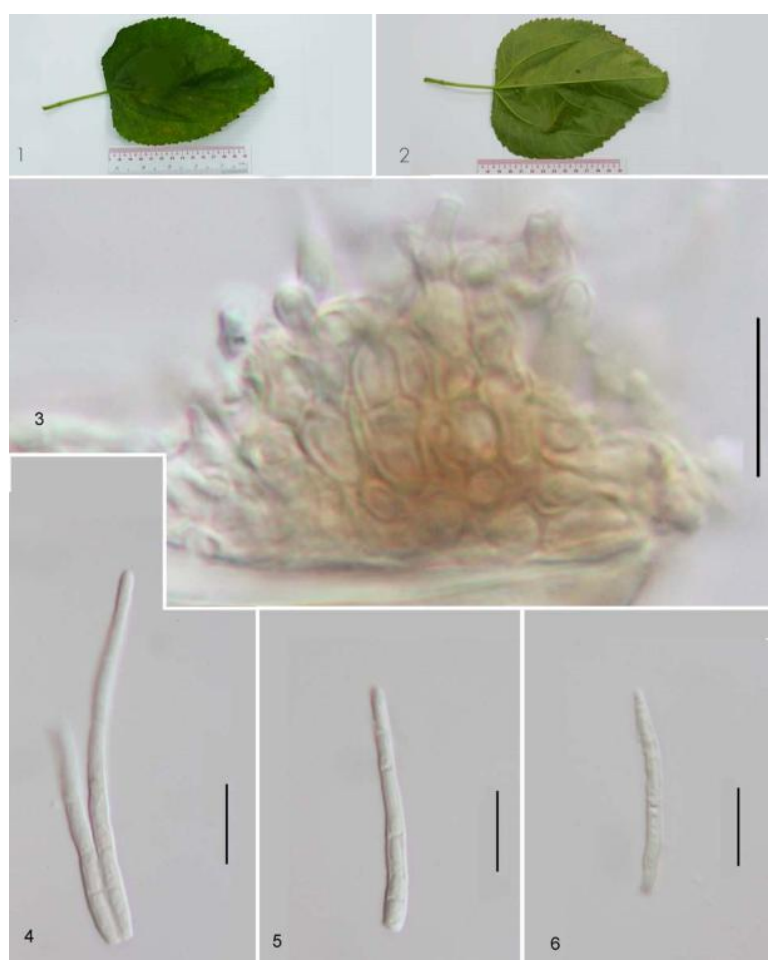

Fig. 77 - Pseudocercospora mori on Molus alba from leaf spots: 1-2. Leaf spots on host leaves (1. upper surface, 2. lower surface). 3. Stroma with attached conidiophores. 4-6. Conidia. Bars: $1-2=10 \mathrm{~mm} .3-6=10 \mu \mathrm{m}$.
Known hosts - Olax scandens Roxb., $O$. wightiana Wall. ex Wight \& Arn., $O$. zeylanica L., Olax sp., Ximenia sp. (Olacaceae). Thailand.

Known distribution - Asia: India, Material examined - Pha Yao Province, Mae Jai District, Mae Puem National Park, on leaves of Olax scandens (Olacaceae), 24 August 2010, P. Phengsintham (MFLU110020).

Notes - The collection from Mae Puem National Park, Pha Yao Province agrees with Pseudocercospora olacicola described by Kamal et al. (1990) [conidiophores 10.5-40.5 $\times$ 2.5-5 $\mu \mathrm{m}$ and conidia $16-30.5 \times 2.5-4 \mu \mathrm{m}]$.

Literature - Crous \& Braun (2003: 297).
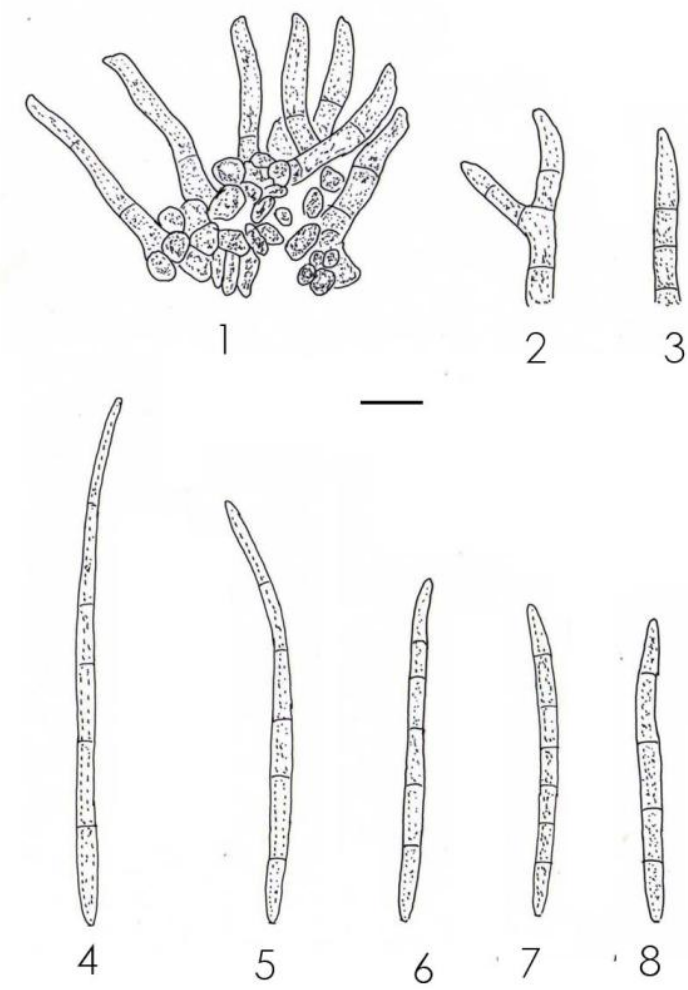

Fig. 78 - Pseudocercospora olacicola on Olax scandens from leaf spots: 1 . Stroma with attached conidiophores. 2-3. Conidio-phores. 4-8. Conidia. Bars: $1-8=10 \mu \mathrm{m}$.

Pseudocercospora oroxyli (A.K. Kar \& M. Mandal) Deighton, Trans. Brit. Mycol. Soc. 88: 388, 1987. 


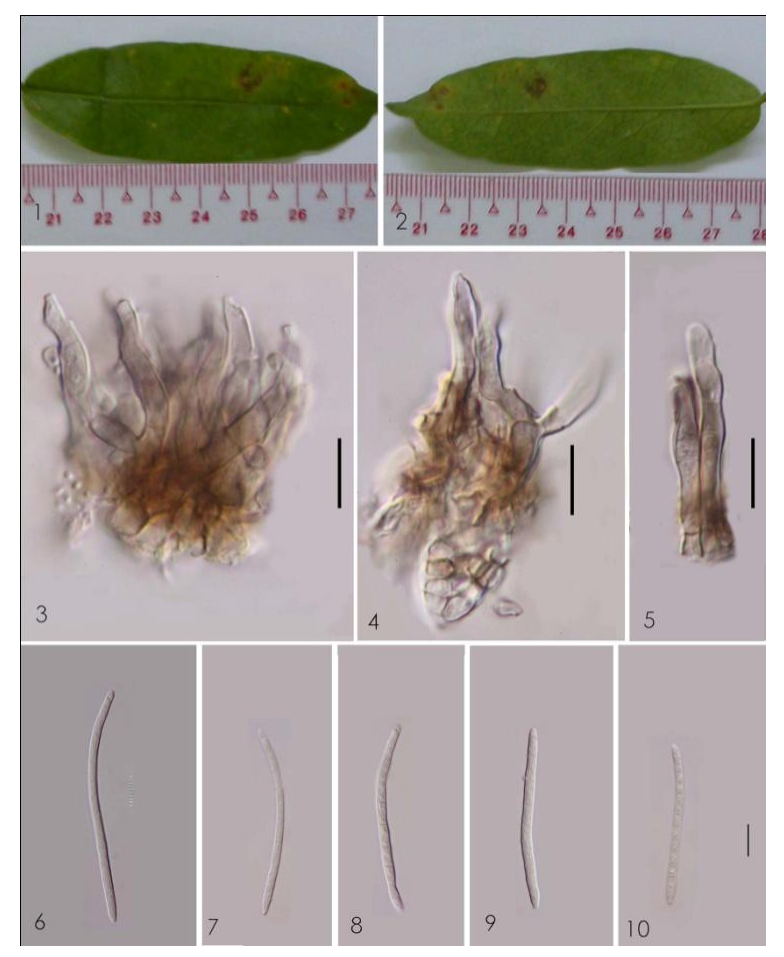

Fig. 79 Pseudocercospora olacicola on Olax scandens from leaf spots: 1-2. Leaf spots on host leaves (1. upper surface, 2. lower surface). 3-4. Stromata with attached conidiophores. 5. Conidiophores. 6-10. Conidia. Bars: $1-2=10$ $\mathrm{mm}, 3$. Not to scale. $3-10=10 \mu \mathrm{m}$.

$\equiv$ Cercospora oroxyli A.K. Kar \& M. Mandal, Trans. Brit. Mycol. Soc. 53: 344, 1969.

Leaf spots circular or irregular, 1-4 mm in diam., brown in the centre, brown to dark brown margin. Colonies hypophyllous, conspicuous, scattered, grey. Mycelium internal; hyphae branched, $2-4 \mu \mathrm{m}$ wide $(\bar{x}=3 \mu \mathrm{m}, \mathrm{n}=$ 8 ), septate, constricted at the septa, distance between septa 5-21 $\mu \mathrm{m}(\bar{x}=11.66 \mu \mathrm{m}, \mathrm{n}=8)$, subhyaline or hyaline, wall $0.3-0.5 \mu \mathrm{m}$ wide $(\bar{x}=0.43 \mu \mathrm{m}, \mathrm{n}=8)$, smooth. Stromata welldeveloped, substomatal, subglobular, 14-21 $\mu \mathrm{m}$ wide $(\bar{x}=17.96 \mu \mathrm{m}, \mathrm{n}=7)$, brown to dark brown, stromatal cells oval, ellipsoidal to angular in outline, 4-6 $\mu \mathrm{m}$ wide $(\bar{x}=4.82 \mu \mathrm{m}$, $\mathrm{n}=20)$, dark brown, wall $0.3-0.5 \mu \mathrm{m}$ wide $(\bar{x}$ $=0.36 \mu \mathrm{m}, \mathrm{n}=20$ ), smooth. Conidiophores fasciculate, arising from stromata (2-7 per fascicle), erect, straight or curved, unbranched, $11-16 \times 3-5 \mu \mathrm{m}(\bar{x}=14.12 \times 3.65 \mu \mathrm{m}, \mathrm{n}=9)$, $0-1$-septate, distance between septa $4-13 \mu \mathrm{m}$ $(\bar{x}=9.41 \mu \mathrm{m}, \mathrm{n}=10)$, pale to moderately olivaceous-brown, paler and narrower towards the apex, wall $0.5-0.8 \mu \mathrm{m}$ wide $(\bar{x}=0.65 \mu \mathrm{m}$, $\mathrm{n}=10)$, smooth. Conidiogenous cells integrated, $12-13 \times 2-4 \mu \mathrm{m}(\bar{x}=12.4 \times 3.23$ $\mu \mathrm{m}, \mathrm{n}=10$ ), pale olivaceous or brown; conidiogenous loci inconspicuous. Conidia solitary, obclavate, straight to moderately curved, $31-75 \times 2-4 \mu \mathrm{m}(\bar{x}=48.47 \times 2.82$ $\mu \mathrm{m}, \mathrm{n}=20), 1-5$-septate, slightly constricted at the septa, pale olivaceous, wall $0.3-0.5 \mu \mathrm{m}$ wide $(\bar{x}=0.35 \mu \mathrm{m}, \mathrm{n}=20)$, smooth, obtuse at the apex, with long obconically truncate base.

Known hosts - Oroxylum indicum (L.) Kurz (Bignoniaceae).

Known distribution - Asia: India, Myanmar, Thailand.

Material examined - Chiang Rai Province, Maechan District, Maechan Village, on leaves of Oroxylum indicum (Bignoniaceae), 18 July 2010, P. Phengsintham (P602).

Notes - In the Thailand collection the conidiophores are 27-70 $\times 5-7 \mu \mathrm{m}$ and the conidia are 14-40 $\times 4-6 \mu \mathrm{m}$, which is similar to those described in Yen (1979).

Literature - Crous \& Braun (2003: 299).

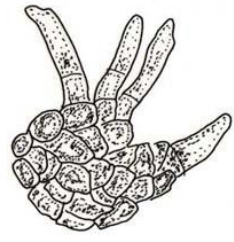

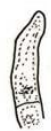

2

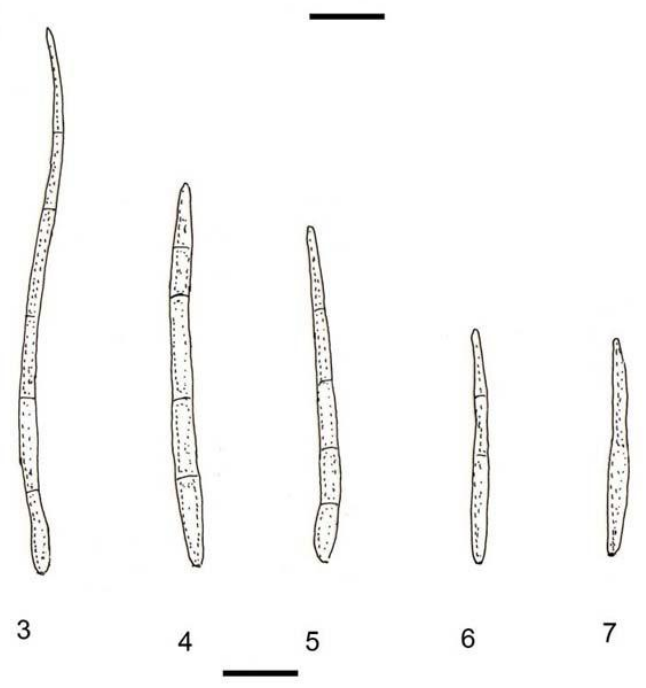

Fig. 80 - Pseudocercospora oroxyli on Oroxylum indicum: 1. Stroma with attached conidiophores. 2. Conidiophore. 3-7. Conidia. Bars: $1-7=10 \mu \mathrm{m}$. 


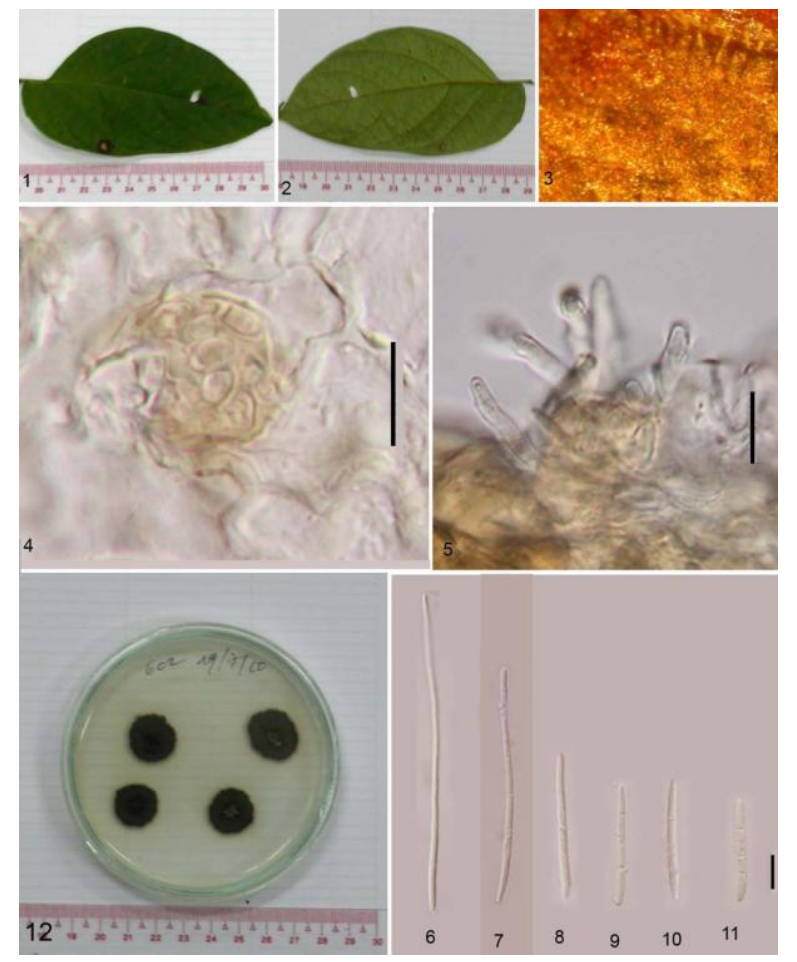

Fig. 81 - Pseudocercospora oroxyli on Oroxylum indicum from leaf spots: 1-2. Leaf spots on host leaves (1. upper surface, 2. lower surface). 3. Colonies. 4. Stroma. 5. Stroma with attached conidiophores. 6-11. Conidia. 12. Culture. Bars: 1$2=10 \mathrm{~mm}$. 3. Not to scale. $4-11=10 \mu \mathrm{m} .12=10$ $\mathrm{mm}$.

Pseudocercospora paederiae Goh \& W.H. Hsieh, in Hsieh and Goh, Cercospora and Similar fungi from Taiwan; 291, 1990.

Figs 82-83.

ECercospora paederiae Sawada, Rep. Gov. Res. Inst. Formosa 87: 84, 1944, nom. inval.

= Cercospora paederiae F.L. Tai, Bull. Chinese Bot. Soc. 2: 56, 1936.

Leaf spots circular or irregular, 1-4 mm in diam., brown in the centre, dark brown margin. Colonies amphigenous, but chiefly hypophyllous. Mycelium internal; hyphae branched, $3-5 \mu \mathrm{m}$ wide $(\bar{x}=4.33 \mu \mathrm{m}, \mathrm{n}=5)$, septate, constricted at the septa, distance between septa $8-10 \mu \mathrm{m}(\bar{x}=9 \mu \mathrm{m}, \mathrm{n}=5)$, subhyaline or hyaline, wall $0.5-0.8 \mu \mathrm{m}$ wide $(\bar{x}=0.6 \mu \mathrm{m}, \mathrm{n}=5)$, smooth. Stromata welldeveloped, substomatal, subglobular, 22-45 $\mu \mathrm{m}$ wide $(\bar{x}=32.3 \mu \mathrm{m}, \mathrm{n}=5)$, brown to dark brown, stromatal cells oval, ellipsoidal to angular in outline, $3-5 \mu \mathrm{m}$ wide $(\bar{x}=4.1 \mu \mathrm{m}$, $\mathrm{n}=8)$, dark brown, wall $0.5-0.8 \mu \mathrm{m}$ wide $(\bar{x}=$
$0.69 \mu \mathrm{m}, \mathrm{n}=8$ ), smooth. Conidiophores solitary or fasciculate, arising from stromata (1-2 per fascicle), erect, straight or curved, unbranched, geniculate, $5-20 \times 3-5 \mu \mathrm{m}(\bar{x}=$ $11 \times 3.67 \mu \mathrm{m}, \mathrm{n}=5), 0-2$-septate, distance between septa $5-13 \mu \mathrm{m}(\bar{x}=8.25 \mu \mathrm{m}, \mathrm{n}=5)$, pale to moderately olivaceous-brown, paler and narrower towards the apex, wall $0.3-0.5 \mu \mathrm{m}$ wide $(\bar{x}=0.35 \mu \mathrm{m}, \mathrm{n}=5)$, smooth. Conidiogenous cells integrated, $5-13 \times 3-4 \mu \mathrm{m}(\bar{x}=$ $8.25 \times 0.35 \mu \mathrm{m}, \mathrm{n}=5$ ), pale olivaceous or brown; conidiogenous loci inconspicuous. Conidia solitary, obclavate, straight to moderately curved, $42-75 \times 2-3 \mu \mathrm{m}(\bar{x}=56 \times$ $2.2 \mu \mathrm{m}, \mathrm{n}=5), 3-6$-septate, slightly constricted at the septa, pale olivaceous, wall $0.25-0.3 \mu \mathrm{m}$ wide $(\bar{x}=0.29 \mu \mathrm{m}, \mathrm{n}=5)$, smooth, obtuse at the apex, with long obconically truncate base.

Known hosts - Paederia chinensis Hance, P. foetida L., P. scandens (Lour.) Merr., P. tomentosa Blume (Rubiaceae).

Known distribution - Asia: China, Japan, Korea, Taiwan and Thailand.

Material examined - Chiang Rai Province, Muanng District, Tadsak water fall, on leaves of Paederia tomentosa (Rubiaceae), 23 December 2009, P. Phengsintham (P499).

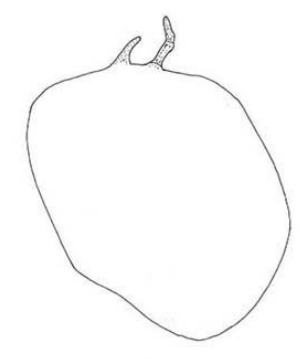

1

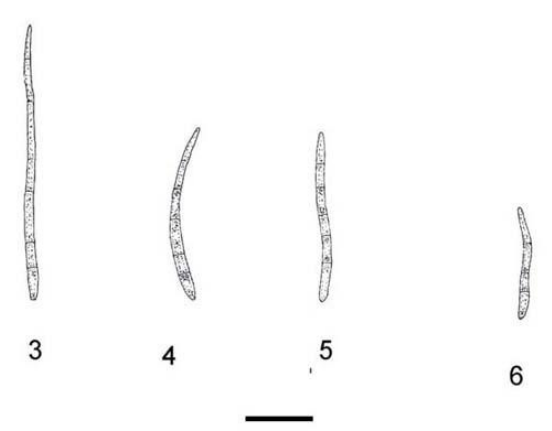

Fig. 82 - Pseudocercospora paederiae on Paederia tomentosa: 1. Stroma with attached conidiophores. 2. Conidiophore. 3-6. Conidia. Bars: $1-6=10 \mu \mathrm{m}$. 


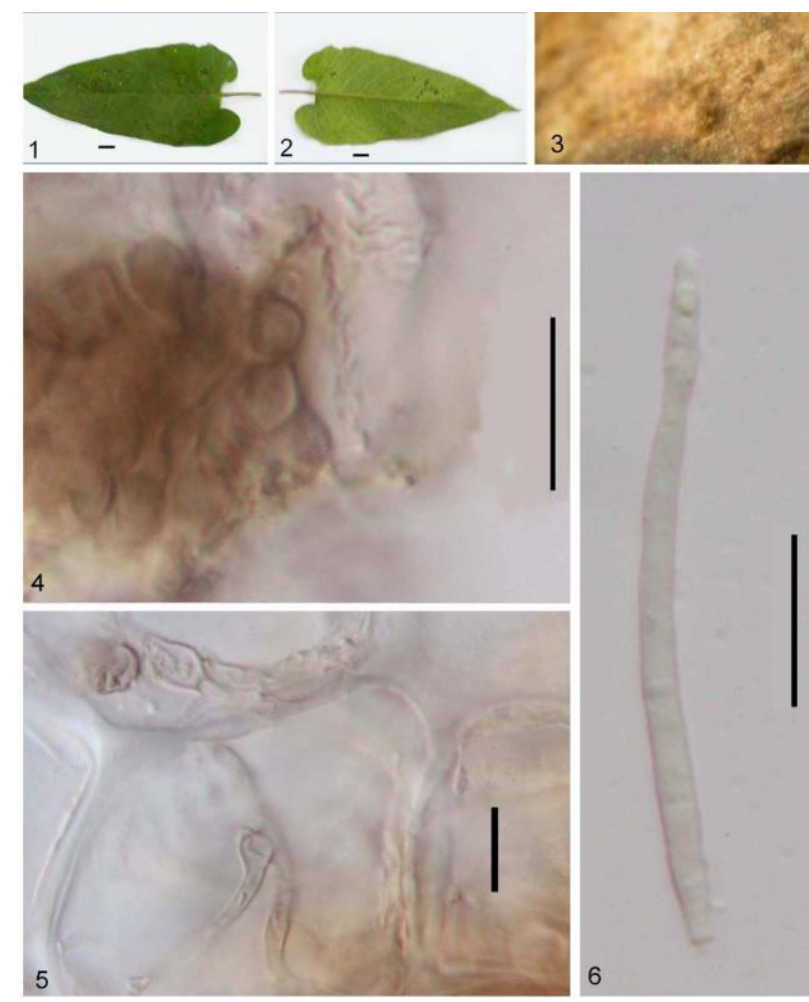

Fig. 83 - Pseudocercospora paederiae on Paederia tomentosa from leaf spots: 1-2. Leaf spots on host leaves (1. upper surface, 2. lower surface). 3. Colonies. 4. Stroma. 5. Internal mycelium. 6. Conidium. Bars: $1-2=10 \mathrm{~mm}$. 3. Not to scale. $4-6=10 \mu \mathrm{m}$.

Notes - The collection from Thailand is similar to the original description of this species, based on material from Taiwan, but there are slight differences in the size of the conidiophores and conidia. The collection from Taiwan has densely fasciculate conidiophores, 20-120 × 3-4 $\mu \mathrm{m}$, subhyaline to pale brown and conidia obclavate, straight to moderately curved, 30-80 × 3.5-5 $\mu \mathrm{m}$, medium olivaceous (Chupp 1954, Hsieh \& Goh 1990, Guo \& Hsieh 1995).

Literature - Chupp (1954: 500); Hsieh \& Goh (1990: 291); Guo \& Hsieh (1995: 291).

Pseudocercospora panacis (Thirum. \& Chupp) Y.L. Guo \& X.J. Liu, Acta Mycol. Sin. 11: 297, 1992.

Figs 84-85.

$\equiv$ Cercospora panacis Thirum. \& Chupp, Mycologia 40: 358, 1948.

$\equiv$ Passalora panacis (Thirum. \& Chupp) Crous \& U. Braun, Mycotaxon 78: 336, 2001.
$=$ Pseudocercospora polysciatispinnatae U. Braun \& Mouch., New Zealand J. Bot. 37: 319, 1999.

Leaf spots circular or irregular, 1-10 $\mathrm{mm}$ in diam., grey-brown in the centre, yellowish brown margin. Colonies hypophyllous. Mycelium internal; hyphae branched, 2-4 $\mu \mathrm{m}$ wide $(\bar{x}=3.33 \mu \mathrm{m}, \mathrm{n}=5)$, septate, constricted at the septa, distance between septa 3-10 $\mu \mathrm{m}(\bar{x}=5 \mu \mathrm{m}, \mathrm{n}=5)$, subhyaline or hyaline, wall $0.3-0.5 \mu \mathrm{m}$ wide $(\bar{x}=0.4 \mu \mathrm{m}, \mathrm{n}$ $=5)$, smooth. Stromata well-developed, substomatal, subglobular, $10-22 \mu \mathrm{m}$ wide $(\bar{x}=$ $16.7 \mu \mathrm{m}, \mathrm{n}=5$ ), brown to dark brown, stromatal cells oval, ellipsoidal to angular in outline, $5-11 \mu \mathrm{m}$ wide $(\bar{x}=7.8 \mu \mathrm{m}, \mathrm{n}=18)$, dark brown, wall $0.5-0.8 \mu \mathrm{m}$ wide $(\bar{x}=0.6$ $\mu \mathrm{m}, \mathrm{n}=18$ ), smooth. Conidiophores solitary or fasciculate, arising from stromata (2-12 per fascicle), erect, straight or curved, simple or occasionally branched, geniculate, $7-35 \times 3-5$ $\mu \mathrm{m}(\bar{x}=39.4 \times 4.21 \mu \mathrm{m}, \mathrm{n}=9), 0-6$-septate, distance between septa $6-13 \mu \mathrm{m}(\bar{x}=10.3 \mu \mathrm{m}$, $\mathrm{n}=30$ ), pale to moderately olivaceous-brown, paler and narrower towards the apex, wall $0.3-$ $0.5 \mu \mathrm{m}$ wide $(\bar{x}=0.45 \mu \mathrm{m}, \mathrm{n}=30)$, smooth. Conidiogenous cells integrated, $6-13 \times 2.5-5$ $\mu \mathrm{m}(\bar{x}=9.63 \times 3.44 \mu \mathrm{m}, \mathrm{n}=5)$, pale olivaceous or brown; conidiogenous loci inconspicuous. Conidia solitary, obclavate, straight to moderately curved, $13-69 \times 3-4 \mu \mathrm{m}(\bar{x}=$ $64.5 \times 3.27 \mu \mathrm{m}, \mathrm{n}=18), 1-5$-septate, slightly constricted at the septa, pale olivaceous, wall $0.3-0.5 \mu \mathrm{m}$ wide $(\bar{x}=0.34 \mu \mathrm{m}, \mathrm{n}=18)$, smooth, obtuse at the apex, with long obconically truncate base.

Known hosts - Kalopanax septemlobus (Thunb. ex A. Murr.) Koidz., Polyscias balfouriana (André) L.H. Bailey, P. fruticosa (L.) Harms, $P$. fulva (Hiern) Harms, $P$. guilfoylei (W. Bull) L.H. Bailey, P. pinnata J.R. Forst. \& G. Forst., Polyscias sp. (Araliaceae).

Known distribution - Africa: Mauritius, Sierra Leone; Asia: Brunei, Cambodia, India, Indonesia, Papua New Guinea, Thailand; South America: Venezuela; Oceania: New Caledonia.

Material examined - Chiang Rai Province, Muang District, Sri Pangsang Village, on leaves of Polyscias balfouriana 


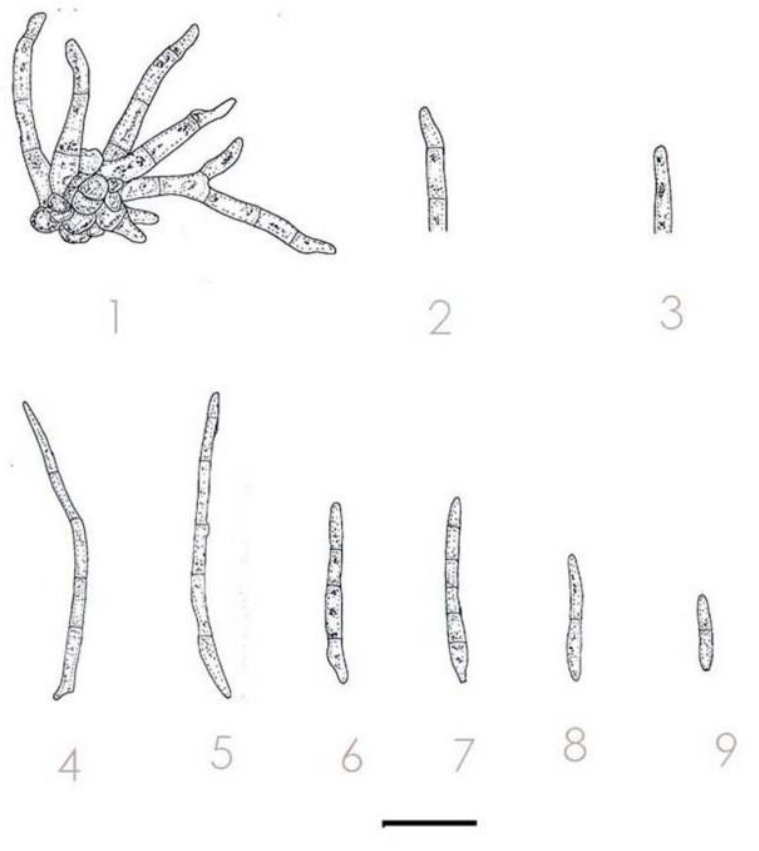

Fig. 84 - Pseudocercospora panacis on Polyscias balfouriana on host leaf: 1. Stroma with attached conidiophores. 2-3. Conidiophore. 4-9. Conidia. Bars: $1-9=10 \mu \mathrm{m}$.

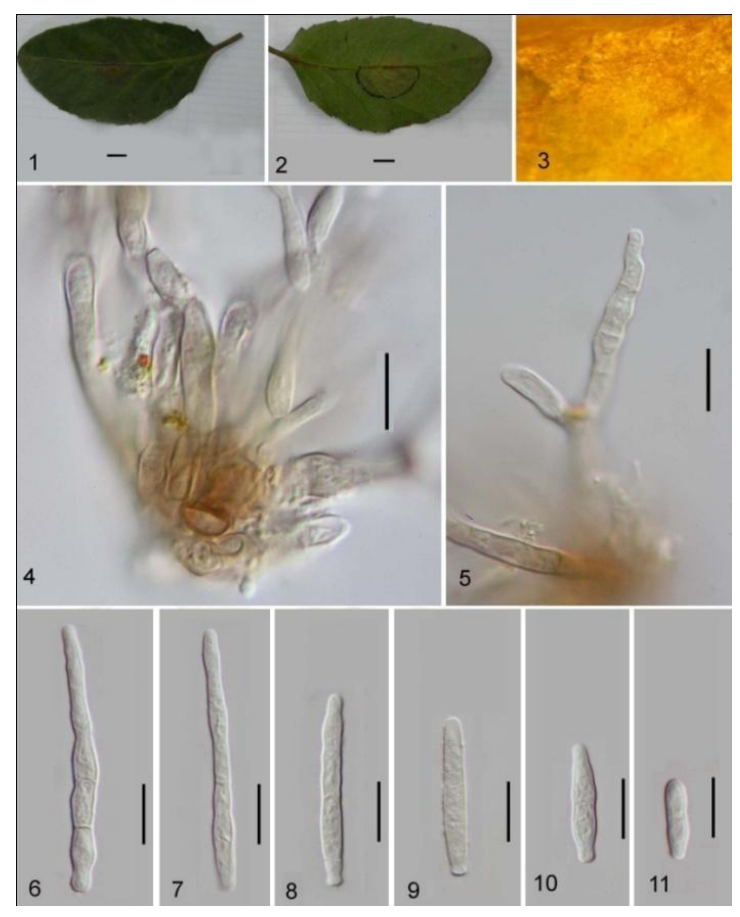

Fig. 85 - Pseudocercospora panacis on Polyscias balfouriana on host leaf: 1. Leaf spots on host leaves (1. upper surface, 2. lower surface). 3. Colonies. 4. Stroma with attached conidiophores. 5. Conidiophore. 6-11. Conidia. Bars: $1-2=10 \mathrm{~mm}, 3$. Not to scale. $4-11=10$ $\mu \mathrm{m}$.
(Araliaceae), 16 January 2010, P. Phengsintham (MFLU10-0322).

Notes - The collection from Sri Pangsang Village, Chiang Rai province agrees well with the description of $P$. panacis in Braun (2003) who described, illustrated and discussed $P$. panacis and $P$. polysciatis (S.H. Sun) J.M. Yen in detail. The latter species is easily distinguishable from $P$. panacis by its very long (up to about $100 \mu \mathrm{m}$ ), frequently branched appendages, sometimes decumbent, developing into superficial hyphae.

Literature - Chupp (1954: 64); Hsieh \& Goh (1990: 357); Guo \& Hsieh (1995: 24); Guo et al. (1998: 38); Braun (2003: 90-92); Crous \& Braun (2003: 305).

Pseudocercospora puderi Deighton, Mycol. Pap. 140: 90, 1976.

Figs 86-87.

$\equiv$ Cercospora puderi B.H. Davis, Mycologia 30: 291, 1938.

Leaf spots subcircular to irregular, 2-10 $\mathrm{mm}$ diam., at first yellowish, later becoming brown in the center, brown to yellowish brown at the margin. Colonies hypophyllous, scattered. Mycelium internal and external hyphae; internal hyphae branched, 2-3 $\mu \mathrm{m}$ wide $(\bar{x}=2.25 \mu \mathrm{m}, \mathrm{n}=7)$, septate, constricted at the septa, distance between septa $7-10 \mu \mathrm{m}$ $(\bar{x}=8.5 \mu \mathrm{m}, \mathrm{n}=5)$, brownish, subhyaline, wall $0.3-0.5 \mu \mathrm{m}$ wide $(\bar{x}=0.4 \mu \mathrm{m}, \mathrm{n}=7$ ), smooth, forming plate-like plectenchymatous stromatic hyphal aggregations; external hyphae branched, $1.5-2 \mu \mathrm{m}$ wide $(\bar{x}=1.76 \mu \mathrm{m}, \mathrm{n}=$ 4), septate, constricted at the septa, distance between septa 5-10 $\mu \mathrm{m}(\bar{x}=7 \mu \mathrm{m}, \mathrm{n}=4)$, brownish, subhyaline, wall $0.3-0.5 \mu \mathrm{m}$ wide $(\bar{x}=0.43 \mu \mathrm{m}, \mathrm{n}=4)$, smooth. Stromata oval to ellipsoidal, $20-36 \mu \mathrm{m}$ diam. $(\bar{x}=28.7 \mu \mathrm{m}, \mathrm{n}=$ $3)$, brown to dark brown, stromatal cells oval, ellipsoidal and angular, 3-6 $\mu \mathrm{m}$ wide $(\bar{x}=4.2$ $\mu \mathrm{m}, \mathrm{n}=13)$, dark brown, wall $0.5-0.8 \mu \mathrm{m}$ wide $(\bar{x}=0.54 \mu \mathrm{m}, \mathrm{n}=13)$, smooth. Conidiophores fasciculate, arising from stromata (4-15 per fascicle), unbranched, not geniculate, $4-7 \times 2-$ $3 \mu \mathrm{m}(\bar{x}=5.5 \times 2.75 \mu \mathrm{m}, \mathrm{n}=15), 0-1$-septate, slightly constricted at the septa, distance between septa 4-7 $\mu \mathrm{m}$ long $(\bar{x}=4.75 \mu \mathrm{m}, \mathrm{n}=$ 16 ), uniformly pale to medium brown, much paler and more narrow toward the tip, wall $0.5-$ 
$0.8 \mu \mathrm{m}(\bar{x}=0.55 \mu \mathrm{m}, \mathrm{n}=15)$, smooth. Conidiogenous cells terminal, $4-7 \times 2-3 \mu \mathrm{m}$ $(\bar{x}=4.75 \times 2.5 \mu \mathrm{m}, \mathrm{n}=9)$, apex obtuse, conidiogenous loci inconspicuous, unthickened, not darkened. Conidia solitary, obclavate, straight to slightly curved, $12-50 \times$ $1-2 \mu \mathrm{m}(\bar{x}=32.66 \times 1.55 \mu \mathrm{m}, \mathrm{n}=9), 1-5-$ septate, pale olivaceous-brown, wall $0.3-0.5$ $\mu \mathrm{m}$ wide $(\bar{x}=0.34 \mu \mathrm{m}, \mathrm{n}=9)$, smooth, tip subacute, base obconically truncate, hila $0.7-$ $1.5 \mu \mathrm{m}$ wide $(\bar{x}=1.22 \mu \mathrm{m}, \mathrm{n}=9)$.

Known hosts - Rosa centifolia L., $R$. davurica Pall., $R$. involucrata Roxb. ex Lindl., $R$. multiflora Thunb., R. odorata (Andrews) Sweet, Rosa sp. (Rosaceae).

Known distribution - Africa: Mauritius, Sierra Leone, Asia: Cambodia, China, Hong Kong, India, Korea, Malaysia, Papua New Guinea, Philippines, Sabah, Thailand; Europe: Cyprus, Netherlands; North America and West Indies: Cuba, Dominican Republ., Haiti, Jamaica, Mexico, USA (FL, GA, TX); South America: Venezuela.

Material examined - Chiang Rai Province, Muang District, Bandu Village, on leaves of Rosa sp. (Rosaceae), 24 August 2010, P. Phengsintham (P622).

Notes - The collection from Thailand differs from the description of $P$. puderi (= Cercospora puderi) in Chupp (1954) [conidiophores 10-30 $\times 2.5-4 \mu \mathrm{m}$, and conidia $20-75 \times 2-3.5 \mu \mathrm{m}]$ and Ellis (1976) [conidiophores 15-16 $\times 2-4 \mu \mathrm{m}$ and conidia $30-80 \times 2-3.5 \mu \mathrm{m}]$ in having shorter conidiophores.

Literature - Chupp (1954: 484); Ellis (1976: 283); Guo \& Hsieh (1995: 282); Crous \& Braun (2003: 341).

Pseudocercospora punicae (Henn.) Deighton, Mycol. Pap. 140: 151, $1976 . \quad$ Figs 88-89. $\equiv$ Cercospora punicae Henn., Bot. Jahrb. Syst. 37: 165, 1906.

Leaf spots subcircular to irregular, $1-4 \mathrm{~mm}$ diam., at first brown, dingy grey to pale tan, brown to dark brown at the margin. Colonies amphigenous, conspicuous. Mycelium internal and external; internal hyphae branched, 2-3 $\mu \mathrm{m}$ wide $(\bar{x}=2.2 \mu \mathrm{m})$, septate, constricted at the septa, distance between septa $6-10 \mu \mathrm{m}(\bar{x}$ $=8.8 \mu \mathrm{m})$, brownish or subhyaline, wall $0.3-$ $0.5 \mu \mathrm{m}$ wide $(\bar{x}=0.34 \mu \mathrm{m})$, smooth, forming
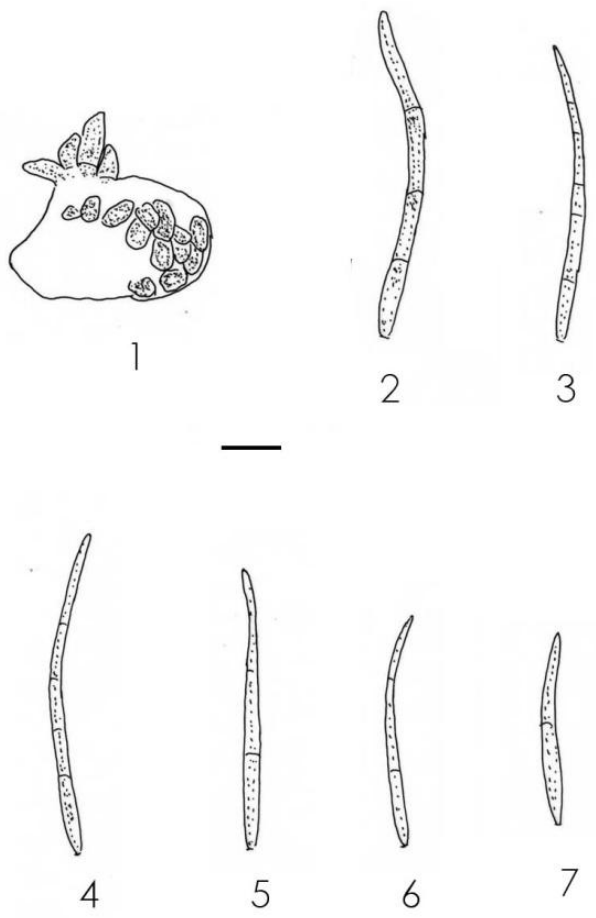

Fig. 86 - Pseudocercospora puderi on Rosa sp. from leaf spots: 1. Stroma with attached conidiophores. 2-7. Conidia. Bars: $1-7=10$ $\mu \mathrm{m}$.

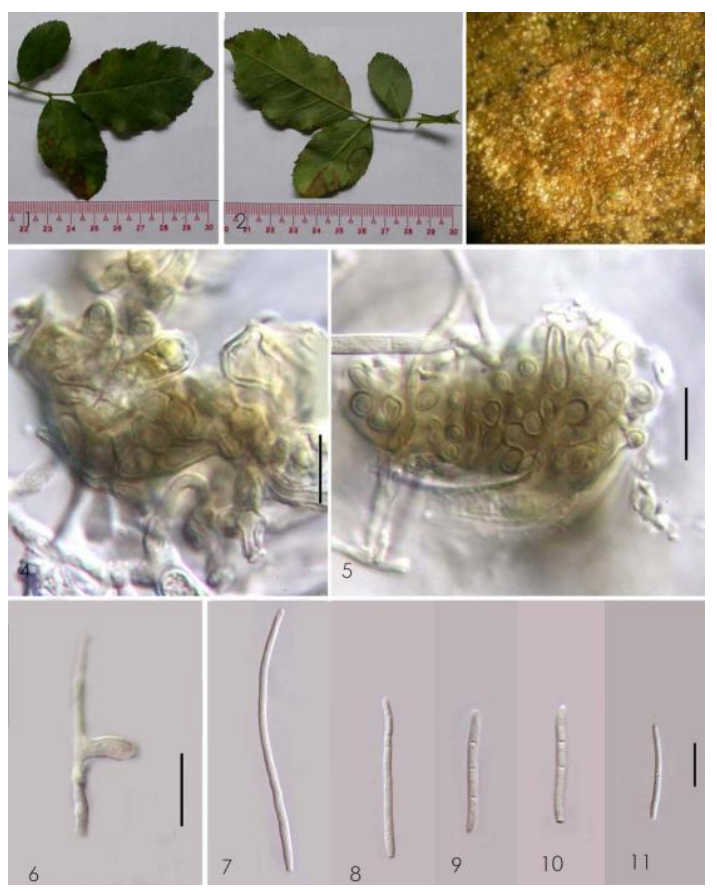

Fig. 87 - Pseudocercospora puderi on Rosa sp. from leaf spots: 1-2. Leaf spots on host leaves (1. upper surface, 2. lower surface). 3. Colonies. 4-5. Stromata with attached conidiophores. 6. External mycelium with attached conidiophore. 7-11. Conidia. Bars: 1$2=10 \mathrm{~mm}, 3$. Not to scale. $4-11=10 \mu \mathrm{m}$. 
plate-like plectenchymatous stromatic hyphal aggregations; external hyphae branched, 2-4 $\mu \mathrm{m}$ wide $(\bar{x}=3 \mu \mathrm{m})$, septate, constricted at the septa, distance between septa 4-8 $\mu \mathrm{m}(\bar{x}=4.7$ $\mu \mathrm{m})$, brownish or subhyaline, wall $0.3-0.5 \mu \mathrm{m}$ wide $(\bar{x}=0.43 \mu \mathrm{m})$, smooth. Stromata oval to ellipsoidal, $10-35 \mu \mathrm{m}$ diam. $(\bar{x}=22.5 \mu \mathrm{m})$, brown to dark brown, stroma cells oval, ellipsoidal to angular, $3-9 \mu \mathrm{m}$ wide $(\bar{x}=6.3$ $\mu \mathrm{m})$, dark brown, wall $0.3-0.5 \mu \mathrm{m}$ wide $(\bar{x}=$ $0.45 \mu \mathrm{m})$, smooth. Conidiophores fasciculate or solitary, arising from stromata (4-40 per fascicle) and external mycelium, geniculate, branched, $7-22 \times 3-4 \mu \mathrm{m}(\bar{x}=13.8 \times 3.28$ $\mu \mathrm{m}), 0-1$-septate, slightly constricted at the septa, distance between septa 5-17 $\mu \mathrm{m}$ long $(\bar{x}$ $=8.33 \mu \mathrm{m}$ ), uniformly pale to medium brown, paler and narrower towards the tip, wall 0.3$0.5 \mu \mathrm{m}(\bar{x}=0.48 \mu \mathrm{m})$, smooth. Conidiogenous cells integrated, terminal, $7-17 \times 3-4 \mu \mathrm{m}(\bar{x}=$ $10 \times 3.11 \mu \mathrm{m})$, apex obtuse; conidiogenous loci inconspicuous, unthickened, not darkened. Conidia solitary, obclavate, straight to slightly curved, $42-72 \times 2-4 \mu \mathrm{m}(\bar{x}=54.23 \times 2.46$ $\mu \mathrm{m}), 2-6$-septate, pale olivaceous-brown, wall $0.3-0.5 \mu \mathrm{m}$ wide $(\bar{x}=0.36 \mu \mathrm{m})$, smooth, tip subacute, base obconically truncate, hila $1.5-2$ $\mu \mathrm{m}$ wide $(\bar{x}=1.55 \mu \mathrm{m})$.

Colonies on PDA after 3 weeks at $25^{\circ} \mathrm{C}$ grey to dark grey, reaching $16-18 \mathrm{~mm}$ diam., hyphae $2-9 \mu \mathrm{m}$ wide $(\bar{x}=5 \mu \mathrm{m})$, septate, constricted at the septa, distance between septa 5-26 $\mu \mathrm{m} \quad(\bar{x}=11.37 \mu \mathrm{m})$, brownish or subhyaline, wall $0.3-1 \mu \mathrm{m}$ wide $(\bar{x}=0.61$ $\mu \mathrm{m})$, smooth. Conidia not formed in culture.

Known hosts - Punica granatum L. (Lythraceae).

Known distribution - Africa: Egypt, Ethiopia, Kenya, Maldives, Mauritius, Mozambique, Sudan, Tanzania, Uganda, Zambia; Asia: Afghanistan, Cambodia, China, Hong Kong, India, Indonesia, Iran, Japan, Laos, Malaysia, Nepal, Pakistan, Philippines, Saudi Arabia, Singapore, Taiwan, Thailand; Europe (Caucasus): Azerbaijan, Georgia; North America and West Indies: Cuba, Dominican Rep., Guatemala, Jamaica, Panama, Puerto Rico, USA (FL, HI, TX), Virgin Islands; South America: Brazil, Colombia, Venezuela.
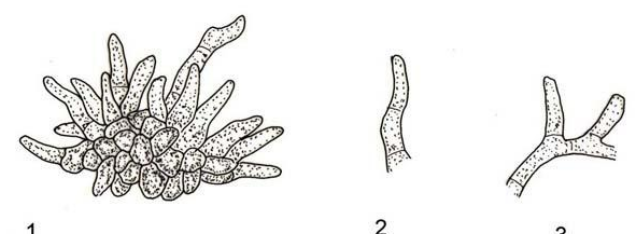

1

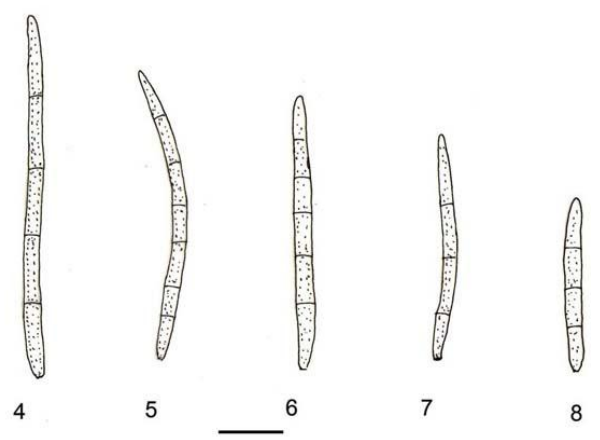

Fig. 88 - Pseudocercospora punicae on Punica granatum: 1. Stroma with attached conidiophores. 2. Conidiophore. 3. External hypha with attached conidiophores. 4-8. Conidia. Bars: $=10 \mu \mathrm{m}$.

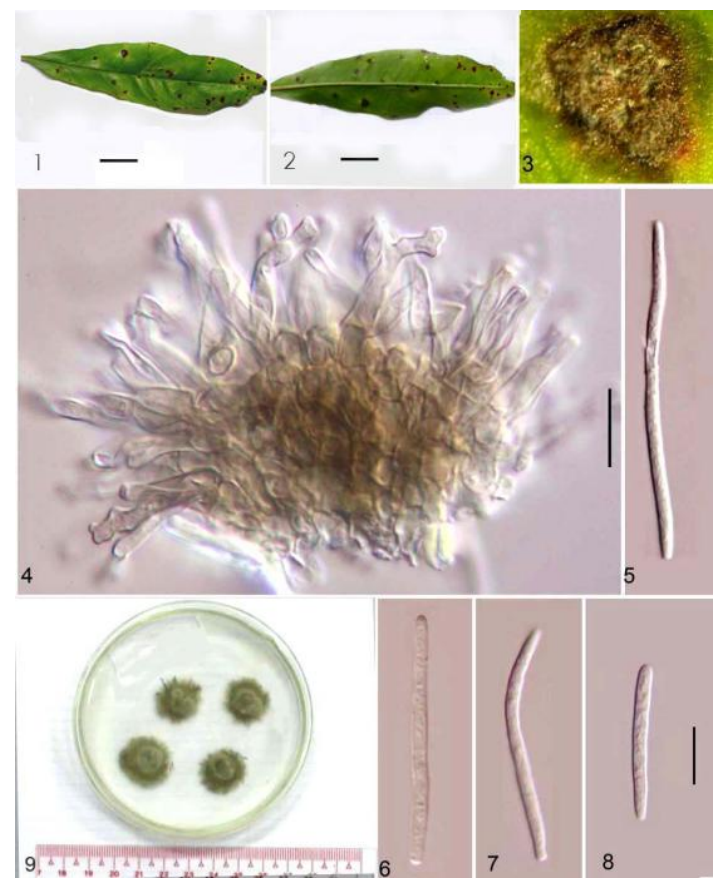

Fig. 89 - Pseudocercospora punicae on Punica granatum from leaf spots: 1-2. Leaf spots on host leaves (1. upper surface, 2. lower surface). 3. Colonies. 4. Stromata with attached conidiophores. 5-8. Conidia. 9. Culture. Bars: 1, 2, $9=10$ mm. 4-8 $=10 \mu \mathrm{m}$. 
Material examined - Chiang Rai Province, Muang District, Ban Du Village, on leaves of Punica granatum (Lythraceae), 1 July 2010, P. Phengsintham (MFLU10-0323). Culture $=$ MFLUCC 11-0284, GenBank accession no JN107998.

Notes - The collection from Thailand differs from Pseudocercospora punicae as described by Hsieh \& Goh (1990) in having geniculate conidiophores.

Literature - Saccardo (1913: 1418); Chupp (1954: 573); Hsieh \& Goh (1990: 271); Guo \& Hsieh (1995: 258); Crous \& Braun (2003: 344).

Pseudocercospora radermachericola Phengsintham, McKenzie, K.D. Hyde \& U. Braun, Mycosphere 1(3): 209, $2010 . \quad$ Figs 90-91.

Leaf spots subcircular to irregular, 1-6 mm diam., at first brown-violet, reddish brown in the centre, brown to dark brown at the margin. Colonies amphigenous, conspicuous. Mycelium internal; hyphae branched, 2-3 $\mu \mathrm{m}$ wide $(\bar{x}=2.66 \mu \mathrm{m}, \mathrm{n}=7)$, septate, constricted at the septa, distance between septa 5-9 $\mu \mathrm{m}(\bar{x}$ $=6.66 \mu \mathrm{m}, \mathrm{n}=7$ ), brownish, subhyaline, wall 0.3-0.5 $\mu \mathrm{m}$ wide $(\bar{x}=0.36 \mu \mathrm{m}, \mathrm{n}=7)$, smooth, forming plate-like plectenchymatous stromatic hyphal aggregations. Stromata oval to ellipsoidal, $8-44 \mu \mathrm{m}$ diam. $(\bar{x}=22.35 \mu \mathrm{m}, \mathrm{n}$ $=6$ ), brown to dark brown, stroma cells oval, ellipsoidal to angular, $3-6 \mu \mathrm{m}$ wide $(\bar{x}=4.8$ $\mu \mathrm{m}, \mathrm{n}=10)$, dark brown, wall $0.5-0.8 \mu \mathrm{m}$ wide $(\bar{x}=0.65 \mu \mathrm{m}, \mathrm{n}=10)$, smooth. Conidiophores fasciculate, arising from stromata (2-46 per fascicle), geniculate, unbranched, 9-26 × 3-5 $\mu \mathrm{m}(\bar{x}=16.94 \times 3.67 \mu \mathrm{m}, \mathrm{n}=9), 0$-2-septate, slightly constricted at the septa, distance between septa $3-25 \mu \mathrm{m}(\bar{x}=10.16 \mu \mathrm{m}, \mathrm{n}=$ 15 ), uniformly pale to medium brown or paler and narrower towards the tip, wall $0.3-0.5 \mu \mathrm{m}$ $(\bar{x}=0.48 \mu \mathrm{m}, \mathrm{n}=15)$, smooth. Conidiogenous cells terminal, $6-25 \times 2-3 \mu \mathrm{m}(\bar{x}=13.52 \times$ $2.49 \mu \mathrm{m}, \mathrm{n}=12$ ), apex obtuse; conidiogenous loci inconspicuous, unthickened, not darkened. Conidia solitary, obclavate, straight to slightly curved, $29-38 \times 2-3 \mu \mathrm{m}(\bar{x}=32.79 \times 2.22$ $\mu \mathrm{m}, \mathrm{n}=15), 0-3$-septate, pale olivaceousbrown, wall $0.3-0.5 \mu \mathrm{m}$ wide $(\bar{x}=0.36 \mu \mathrm{m}, \mathrm{n}$ $=15$ ), smooth, tip subacute, base obconically truncate, hila $1-2 \mu \mathrm{m}$ wide $(\bar{x}=1.18 \mu \mathrm{m}, \mathrm{n}=$ $15)$.
Colonies on PDA after 3 weeks at $25^{\circ} \mathrm{C}$ dark-grey, reaching 4-7 mm diam., hyphae 2-6 $\mu \mathrm{m}$ wide $(\bar{x}=4.75 \mu \mathrm{m}, \mathrm{n}=10)$, septate, constricted at the septa, distance between septa 9-16 $\mu \mathrm{m}(\bar{x}=12.75 \mu \mathrm{m}, \mathrm{n}=10)$, brownish or subhyaline, wall $0.3-0.8 \mu \mathrm{m}$ wide $(\bar{x}=0.52$ $\mu \mathrm{m}, \mathrm{n}=10$ ), smooth. Conidia not formed in culture.

Known host - Radermachera ignea (Kurz) Steenis (Bignoniaceae).

Known distribution - Asia: Thailand.

Material examined: Chiang Rai Province, Muang District, Sri Pangsang Village, on leaves of Radermachera ignea (Bignoniaceae), 12 August 2010, P. Phengsintham (MFLU10-0406, holotype).

Notes - Cercospora radermacherae Boedijn (Boedijn, 1961) was described from Indonesia on Radermachera glandulosa (as "glandulata"). Braun (2001) re-examined type material of this species assigned it to Pseudocercospora and published a description and illustration. $P$. radermacherae is quite distinct from the new species on Radermachera ignea by its long $(25-80 \times 2-4 \mu \mathrm{m})$, pluriseptate, densely fasciculate, often almost synnematous conidiophores and longer conidia, $30-70 \times 2-4 \mu \mathrm{m}$. Radermachera ignea is not closely allied to $R$. glandulosa. In the "Flora of China" (Wu \& Robens, 1988), the accepted name of this species is Mayodendron igneum.

Literatures - Boedijn (1961: 411-436); Wu \& Robens (1988); Braun (2001: 419-436).

Pseudocercospora riachueli var. horiana (Togashi \& Katsuki) U. Braun \& Crous, in Crous \& Braun, CBS Biodiversity Series 1: 354, 2003.

Figs 92-93.

$\equiv$ Cercospora horiana Togashi \& Katsuki, Sci. Rep. Yokohama Natl. Univ., Sect. 2, Biol. Sci., 1: 4, 1952.

= Pseudocercospora cissi Bagyan., U. Braun \& Jagad., Mycotaxon 45: 106, 1992. =Pseudocercospora ampelocissi P.N. Singh, in herb.

Leaf spots circular to irregular, $1-5 \mathrm{~mm}$ in diam., brown to grey-brown in the center, and dark brown margin. Colonies amphigenous, scattered. Mycelium internal and external; internal hyphae branched, 2-4 $\mu \mathrm{m}$ wide $(\bar{x}=2.8 \mu \mathrm{m}, \mathrm{n}=5)$, septate, constricted 


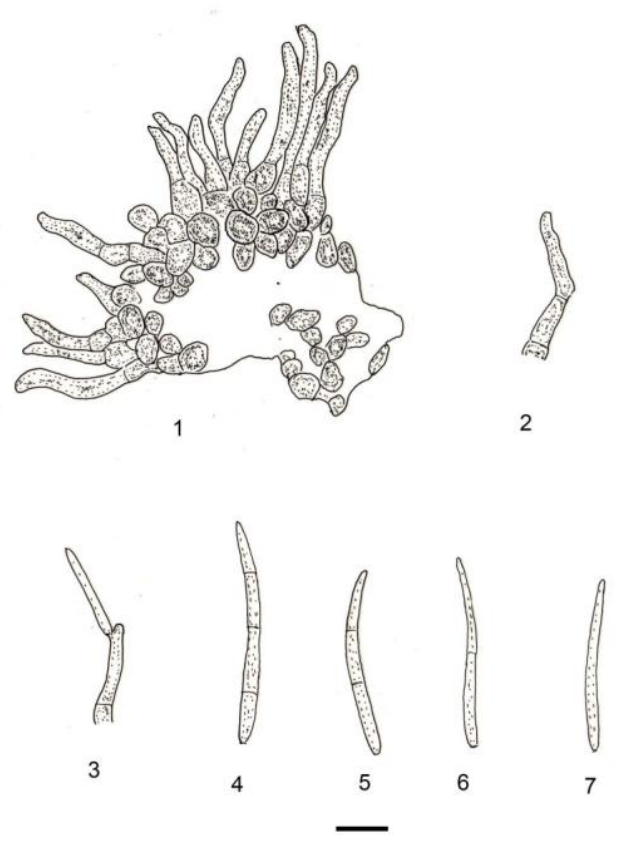

Fig. 90 - Pseudocercospora radermachericola on Radermachera ignea: 1. Stroma with attached conidiophores. 2. Conidiophore. 3. Conidiophore with attached young conidium. 4-7. Conidia. Bars: $=10 \mu \mathrm{m}$.

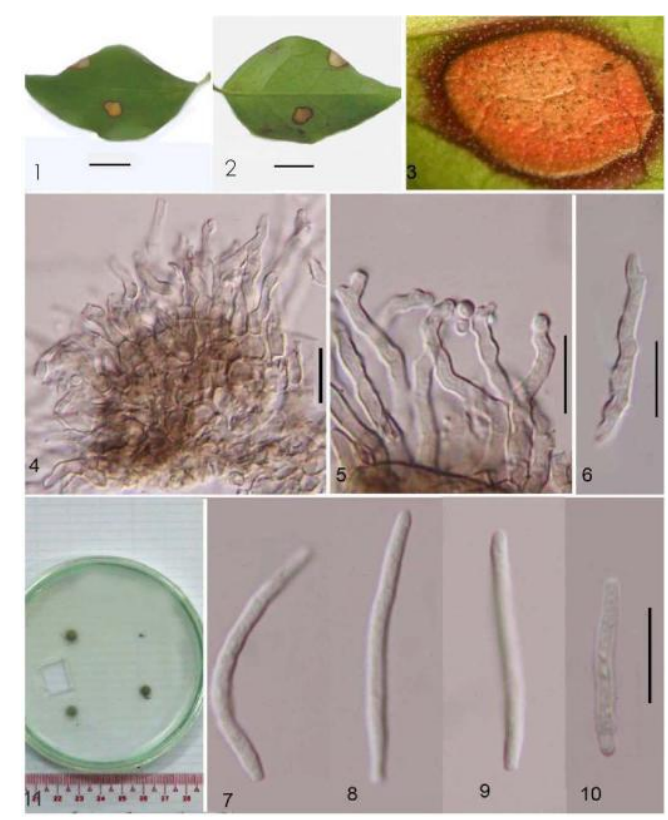

Fig. 91 - Pseudocercospora radermachericola on Radermachera ignea from leaf spots: 1, 2. Leaf spots on host leaves (1. upper surface, 2. lower surface). 3. Colonies. 4. Stroma with attached conidiophores. 5-6. Conidiophores. 7-10. Conidia. 11. Culture. Bars: $1-2,11=10$ mm. $4-10=10 \mu \mathrm{m}$. at the septa, distance between septa 6-15 $\mu \mathrm{m}$ $(\bar{x}=9.2 \mu \mathrm{m}, \mathrm{n}=5)$, subhyaline or hyaline, wall $0.3-0.5 \mu \mathrm{m}$ wide $(\bar{x}=0.46 \mu \mathrm{m}, \mathrm{n}=5)$, smooth; external hyphae branched, 2-3 $\mu \mathrm{m}$ wide $(\bar{x}=2.5 \mu \mathrm{m}, \mathrm{n}=7)$, septate, constricted at the septa, distance between septa 8-10 $\mu \mathrm{m}$ $(\bar{x}=9 \mu \mathrm{m}, \mathrm{n}=7)$, subhyaline or hyaline, wall $0.3-0.5 \mu \mathrm{m}$ wide $(\bar{x}=0.4 \mu \mathrm{m}, \mathrm{n}=7)$, smooth. Stromata well-developed, substomatal, subglobular, $20-50 \mu \mathrm{m}$ wide $(\bar{x}=30 \mu \mathrm{m}, \mathrm{n}=4)$, brown to dark brown, stromatal cells oval, ellipsoidal to angular in outline, $2-3 \mu \mathrm{m}$ wide $(\bar{x}=2.7 \mu \mathrm{m}, \mathrm{n}=15)$, dark brown, wall $0.5-0.8$ $\mu \mathrm{m}$ wide $(\bar{x}=0.55 \mu \mathrm{m}, \mathrm{n}=15)$, smooth. Conidiophores fasciculate, arising from stromata (8-45 per fascicle), erect, straight or curved, unbranched, $10-17 \times 2-4 \mu \mathrm{m}(\bar{x}=$ $14.7 \times 2.86 \mu \mathrm{m}, \mathrm{n}=13), 0-1$-septate, distance between septa $6-17 \mu \mathrm{m}(\bar{x}=11.6 \mu \mathrm{m}, \mathrm{n}=15)$, pale to moderately olivaceous-brown, paler and narrower towards the apex, wall $0.5-0.8 \mu \mathrm{m}$ wide $(\bar{x}=0.58 \mu \mathrm{m}, \mathrm{n}=15)$, smooth. Conidiogenous cells integrated, $10-17 \times 2-4$ $\mu \mathrm{m}(\bar{x}=14 \times 2.8 \mu \mathrm{m}, \mathrm{n}=5)$, pale olivaceous or brown; conidiogenous loci inconspicuous. Conidia solitary, obclavate, straight to moderately curved, $21-67 \times 1.5-3 \mu \mathrm{m}(\bar{x}=$ $50.71 \times 1.85 \mu \mathrm{m}, \mathrm{n}=13), 3-9$-septate, slightly constricted at the septa, pale olivaceous, wall $0.3-0.5 \mu \mathrm{m}$ wide $(\bar{x}=0.36 \mu \mathrm{m}, \mathrm{n}=13)$, smooth, obtuse at the apex, with long obconically truncate base.

Known Hosts - Ampelocissus latifolia (Roxb.) Planch., Cissus sp., Parthenocissus tricuspidata (Siebold \& Zucc.) Planch., Vitis vinifera L. (Vitaceae). Thailand.

Distribution - Asia: India, Japan and

Material examined - Chiang Rai Province, Muang District, Ban Du Village, on leaves of Vitis vinifera, 24 August 2010, P. Phengsintham (P632).

Notes $-P$. riachueli var. horiana differs from var. riachueli in forming superficial hyphae. The collection from Thailand agrees well with $P$. riachueli except for narrower conidia. Vitis vinifera is a new host for $P$. riachueli var. horiana.

Literature - Crous \& Braun (2003: $354)$. 

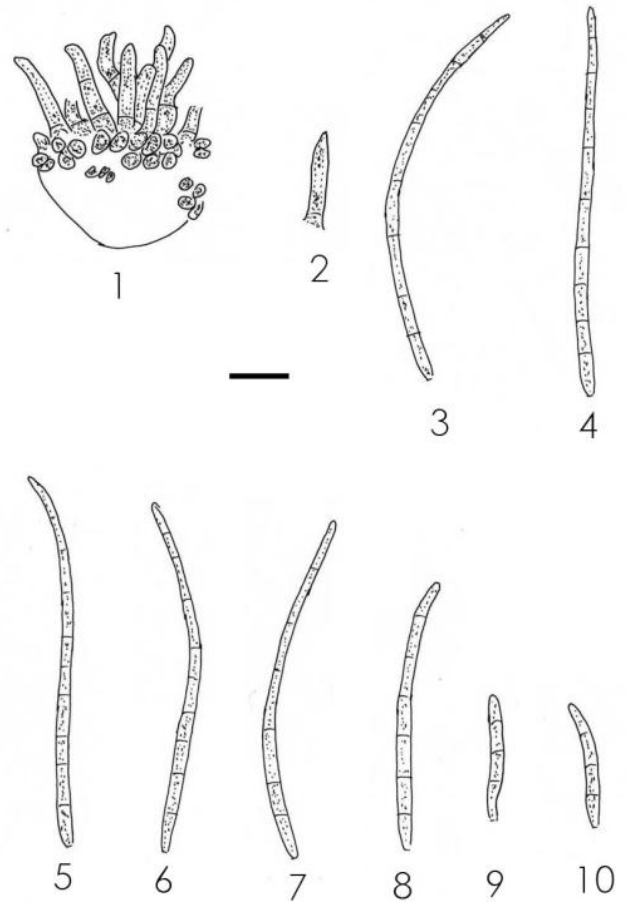

Fig. 92 - Pseudocercospora riachueli var. horiana on Vitis vinifera from leaf spots: 1. Stroma with attached conidiophores. 2. Conidiophore. $3-10$. Coniidia. Bar $=10 \mu \mathrm{m}$.

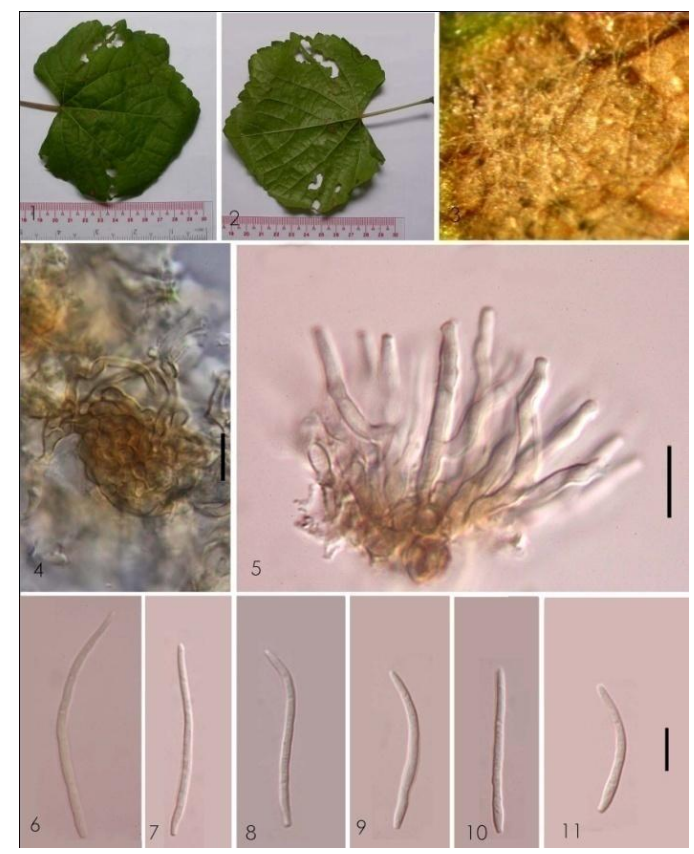

Fig. 93 - Pseudocercospora riachueli var. horiana on Vitis vinifera from leaf spots: 12. Leaf spots on host leaves (1. upper surface, 2. lower surface). 3. Colonies. 4. Stroma. 5. Stroma with attached conidiophores. 6-11. Conidia. Bars 1-2 = 10 $\mathrm{mm}, 4-11=10 \mu \mathrm{m}$.
Pseudocercospora scopariicola (J.M. Yen) Deighton, Mycol. Pap. 140: 152, 1976.

Figs 94-95.

三 Cercospora scopariicola J.M. Yen,

Bull. Trimestriel Soc. Mycol. France 84: 11, 1968.

Leaf spots circular, 2-6 $\mathrm{mm}$ in diam., yellow to grey-yellow in the center, and yellowish green margin. Colonies amphigenous, but chiefly hypophyllous. Mycelium internal, inconspicuous. Stromata welldeveloped, substomatal, subglobular, 10-22 $\mu \mathrm{m}$ wide $(\bar{x}=16.7 \mu \mathrm{m}, \mathrm{n}=6)$, brown to dark brown, stromatal cells oval, ellipsoidal to angular in outline, $3-6 \mu \mathrm{m}$ wide $(\bar{x}=4.4 \mu \mathrm{m}$, $\mathrm{n}=30)$, dark brown, wall $0.5-0.8 \mu \mathrm{m}$ wide $(\bar{x}$ $=0.52 \mu \mathrm{m}, \mathrm{n}=30$ ), smooth. Conidiophores fasciculate, arising from stromata (2-14 per fascicle), erect, straight or curved, unbranched, geniculate, $13-34 \times 3-4 \mu \mathrm{m}(\bar{x}=20 \times 3.33$ $\mu \mathrm{m}, \mathrm{n}=12), 0-3$-septate, distance between septa 5-20 $\mu \mathrm{m}(\bar{x}=11.8 \mu \mathrm{m}, \mathrm{n}=30)$, pale to moderately olivaceous-brown, paler and narrower towards the apex, wall $0.5-0.8 \mu \mathrm{m}$ wide $(\bar{x}=0.58 \mu \mathrm{m}, \mathrm{n}=30)$, smooth. Conidiogenous cells integrated, $10-19 \times 2-4$ $\mu \mathrm{m}(\bar{x}=14.3 \times 3.11 \mu \mathrm{m}, \mathrm{n}=9)$, pale olivaceous or brown; conidiogenous loci inconspicuous. Conidia solitary, obclavate, straight to moderately curved, $13-85 \times 1.5-3$ $\mu \mathrm{m}(\bar{x}=55.42 \times 2.35 \mu \mathrm{m}, \mathrm{n}=7), 0-7$-septate, slightly constricted at the septa, pale olivaceous, wall $0.3-0.5 \mu \mathrm{m}$ wide $(\bar{x}=0.39$ $\mu \mathrm{m}, \mathrm{n}=7$ ), smooth, obtuse at the apex, with long obconically truncate base, $1-2 \mu \mathrm{m}$ wide $(\bar{x}=1.71 \mu \mathrm{m}, \mathrm{n}=7)$.

Known hosts - Scoparia dulcis L., Vernonia sp. (Plantagiaceae).

Known distribution - Asia: China, India, Laos, Taiwan, Thailand.

Material examined - Chiang Rai Province, Muang District, Sri Pangsang Village, on leaves of Scoparia dulcis (Plantaginaceae), 11 June 2011, P. Phengsintham (P642).

Notes - The collection from Thailand differs from the description of $P$. scopariicola published by Guo \& Hsieh (1995) [conidiophores $10-50 \times 3-5.5 \mu \mathrm{m}$ and conidia $45-170$ $\times 3-4 \mu \mathrm{m}]$ in having a shorter of conidiophores and conidia. 

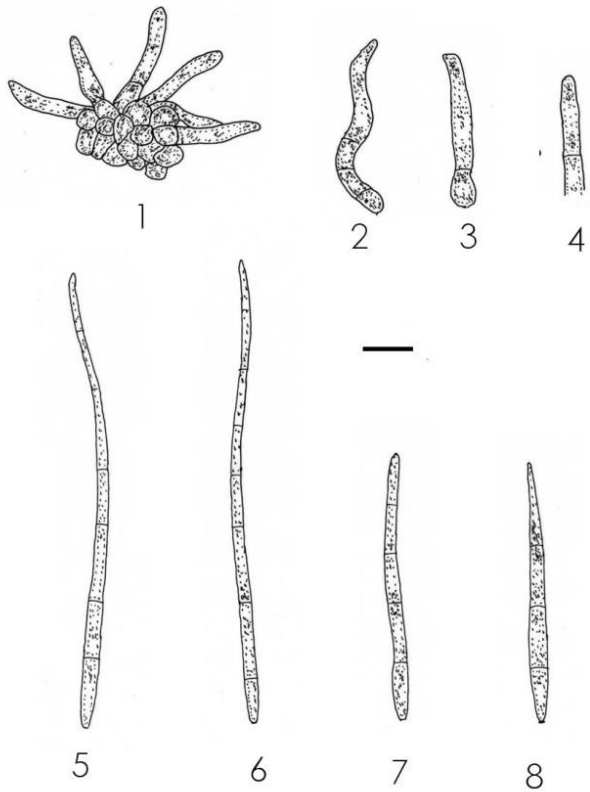

Fig. 94 - Pseudocercospora scopariicola on Scoparia dulcis from leaf spots: 1. Stroma with attached conidiophores. 3-4. Conidiophores. 6-9. Conidia. Bars: $1-8=10 \mu \mathrm{m}$.

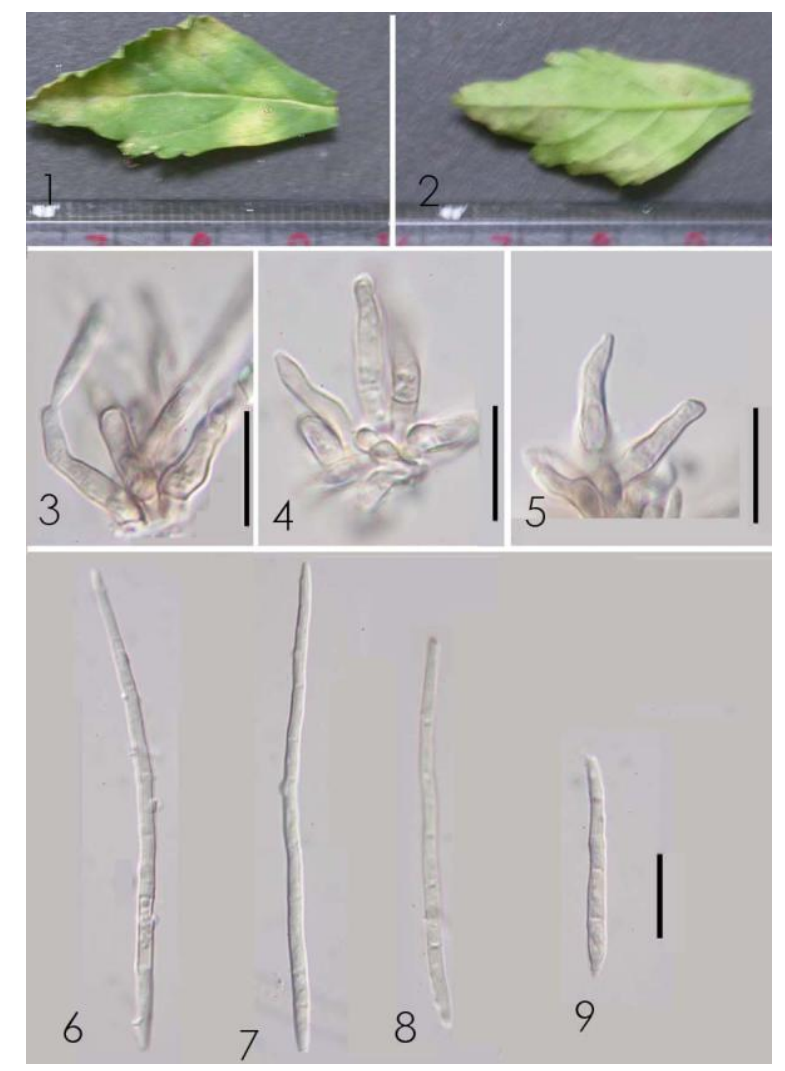

Fig. 95 - Pseudocercospora scopariicola on Scoparia dulcis from leaf spots: 1-2. Leaf spots on host leaves (1. upper surface, 2. lower surface). 3-5. Stromata with attached conidiophores. 6-9. Conidia. Bars: $1-2=10 \mathrm{~mm}$. 3-9 $=10 \mu \mathrm{m}$.
Literature - Yen \& Lim (1980: 185); Hsieh \& Goh (1990: 309); Guo \& Hsieh (1995: 311); Crous \& Braun (2003: 370).

\section{Pseudocercospora timorensis (Cooke)}

Deighton, Mycol. Pap. 140: 154, 1976.

Figs 96-97.

$$
\equiv \text { Cercospora timorensis Cooke, }
$$

Grevillea 12: 38, 1883.

$$
\text { = Ramularia batatas Racib., Paras. }
$$

Algen Pilze Javas, Batavia I: 35, 1900.

$=$ Cercospora batatae Zimm., Ber.

Land. Forstw. Deutsch Ostafrikas 2: 28, 1904.

$=$ Cercospora batatae Henn., Bot. Syst. 38: 118, 1907.

$=$ Cercospora ipomoeae-purpureae J.M. Yen, Rev. Mycol. 30: 173. 1965.

$\equiv$ Pseudocercospora ipomoeaepurpureae (J.M. Yen) J.M. Yen, in Yen and Lim, Gard. Bull. Singapore 33: 177, 1980.

Leaf spots circular, 2-8 $\mathrm{mm}$ in diam., brown to dark brown in the center, and dark brown margin. Colonies amphigenous, but chiefly hypophyllous. Mycelium internal; hyphae branched, $2-4 \mu \mathrm{m}$ wide $(\bar{x}=2.8 \mu \mathrm{m}, \mathrm{n}$ $=5$ ), septate, constricted at the septa, distance between septa $8-11 \mu \mathrm{m}(\bar{x}=9 \mu \mathrm{m}, \mathrm{n}=5)$, subhyaline or hyaline, wall $0.5-0.8 \mu \mathrm{m}$ wide $(\bar{x}=0.56 \mu \mathrm{m}, \mathrm{n}=5)$, smooth. Stromata welldeveloped, substomatal, subglobular, 8-26 $\mu \mathrm{m}$ wide $(\bar{x}=16 \mu \mathrm{m}, \mathrm{n}=5)$, brown to dark brown, stromatal cells oval, ellipsoidal to angular in outline, $5-8 \mu \mathrm{m}$ wide $(\bar{x}=6.2 \mu \mathrm{m}, \mathrm{n}=30)$, dark brown, wall $0.5-1 \mu \mathrm{m}$ wide $(\bar{x}=0.72$ $\mu \mathrm{m}, \quad \mathrm{n}=30$ ), smooth. Conidiophores fasciculate, arising from stromata (2-7 per fascicle), erect, straight or curved, unbranched, geniculate, $11-45 \times 2-6 \mu \mathrm{m}(\bar{x}=22.6 \times 4 \mu \mathrm{m}$, $\mathrm{n}=8), 0-3$-septate, distance between septa 7$14 \mu \mathrm{m}(\bar{x}=10.8 \mu \mathrm{m}, \mathrm{n}=15)$, pale to moderately olivaceous-brown, paler and narrower towards the apex, wall $0.5-0.8 \mu \mathrm{m}$ wide $(\bar{x}=0.65 \mu \mathrm{m}, \mathrm{n}=15)$, smooth. Conidiogenous cells integrated, 7-14 $\times 3-4 \mu \mathrm{m}$ $(\bar{x}=11.9 \times 3.75 \mu \mathrm{m}, \mathrm{n}=8)$, pale olivaceous or brown; conidiogenous loci inconspicuous. Conidia solitary, obclavate, straight to moderately curved, $30-77 \times 3-4 \mu \mathrm{m}(\bar{x}=57 \times$ $3.5 \mu \mathrm{m}, \quad \mathrm{n}=12), \quad 3-8$-septate, slightly constricted at the septa, pale olivaceous, wall $0.3-0.5 \mu \mathrm{m}$ wide $(\bar{x}=0.48 \mu \mathrm{m}, \mathrm{n}=12)$, 
smooth, obtuse at the apex, with long obconically truncate base.

Colonies on PDA after 3 weeks at $25^{\circ} \mathrm{C}$ grey in the center and dark brown margin, surface ridged and smooth, 6-10 $\mathrm{mm}$ diam.

Known hosts - Calystegia sp., Ipomoea acuminata (Vahl) Roem. \& Schultes, I. asarifolia (Desr.) Roem., I. batatas (L.) Poir, I. biloba Forssk, I. campanulata L., I. purpurea (L.) Roth., I. reptans Forsk., I. setifera Poir, Ipomoea sp., Operculina sp. (Convolvulaceae).

Known distribution - Africa: Gabon, Ghana, Mauritius, Sierra Leone, South Africa, Sudan, Tanzania, Uganda; Asia: Bangladesh, Brunei, China, Hong Kong, India, Indonesia, Japan, Laos, Malaysia, Nepal, Papua New Guinea, Philippines, Singapore, Taiwan, Thailand; North America and West Indies: Panama, Trinidad and Tobago; Oceania: Fiji, Kiribati, Micronesia, Palau, Samoa, Solomon Islands, St. Lucia, Tonga, Vanuatu; South America: Brazil, Ecuador, Venezuela.

Material examined - Chiang Rai Province, Muang District, Sri Pangsang Village, on leaves of Operculina sp. (Convolvulaceae), 15 September 2009, P. Phengsintham (P455). GenBank accession no (LSU, KC677937).

Notes - The collection from Thailand agrees well with the descriptions of Pseudocercospora timorensis in Chupp (1954), Ellis (1976), Hsieh \& Goh (1990) and Guo \& Hsieh (1995).

Literature - Chupp (1954: 173); Ellis (1976: 253); Hsieh \& Goh (1990: 91); Guo \& Hsieh (1995: 75); Crous \& Braun (2003: 404).

Pseudocercospora tremicola (J.M. Yen) Deighton [as 'trematicola'], Mycol. Pap. 140: 154, 1976.

Figs 98-99.

三Cercospora tremicola J.M. Yen, Bull. Trimestiel Soc. Mycol. France 86: 752, (1970) 1971.

= Cercospora tremae-guineensis J.M. Yen \& Gilles (as 'trematis-guineensis'), Bull. Trimestiel Soc. Mycol. France 90: 322, (1974) 1975.

$\equiv$ Pseudocercospora tremae-guineensis (J.M. Yen \& Gilles) J.M. Yen, Bull. Trimestiel Soc. Mycol. France 94: 283, (1978) 1979.

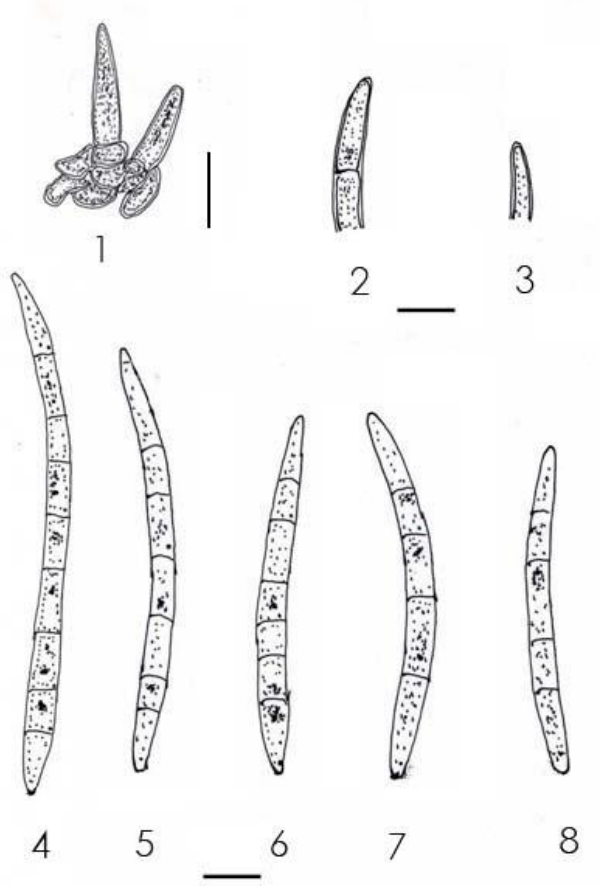

Fig. 96 - Pseudocercospora timorensis on Operculina sp. from leaf spots: 1. Stroma with attached conidiophores. 2-3. Conidiophores. 4-8. Conidia. Bars: $1-8=$ $10 \mu \mathrm{m}$.

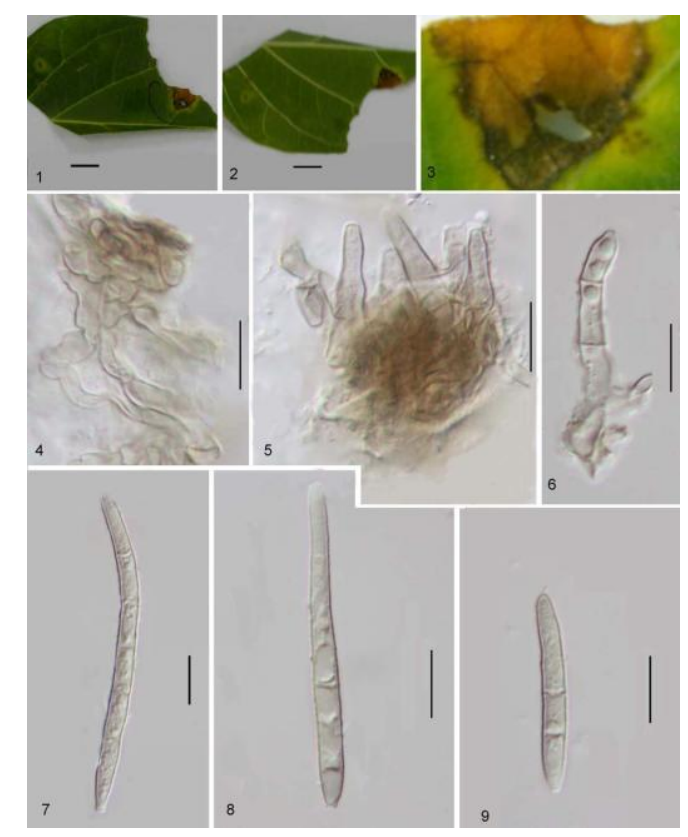

Fig. 97 - Pseudocercospora timorensis on Operculina sp. from leaf spots: 1-2. Leaf spots on host leaves (1. upper surface, 2. lower surface). 3. Colonies. 4. Internal hyphae. 5. Stroma with attached conidiophores. 6 . Conidiophore. 7-9. Conidia. Bars: $1-2=10$ mm. 3. Not to scale. 4-9 = $10 \mu \mathrm{m}$. 
Leaf spots circular to irregular, 2-5 mm in diam., brown in the center, and dark brown margin. Colonies amphigenous, but chiefly hypophyllous. Mycelium internal, inconspicuous and external; external hyphae branched, $3-4 \mu \mathrm{m}$ wide $(\bar{x}=3.6 \mu \mathrm{m}, \mathrm{n}=7)$, septate, constricted at the septa, distance between septa $10-17 \mu \mathrm{m}(\bar{x}=14 \mu \mathrm{m}, \mathrm{n}=7)$, subhyaline or hyaline, wall $0.5-0.8 \mu \mathrm{m}$ wide $(\bar{x}=0.7 \mu \mathrm{m}, \mathrm{n}=7)$, smooth. Stromata lacking or very small, composed of 2-3 stromatic cells. Conidiophores solitary or fasciculate, arising from stromata (2-3 per fascicle) and arising from external hyphae, erect, straight or curved, unbranched, $30-110 \times 3-4 \mu \mathrm{m}(\bar{x}=81.3 \times$ $3.75 \mu \mathrm{m}, \mathrm{n}=13)$, 3-6-septate, distance between septa $12-18 \mu \mathrm{m}(\bar{x}=15.3 \mu \mathrm{m}, \mathrm{n}=$ 30 ), pale to moderately olivaceous-brown, paler and narrower towards the apex, wall $0.5-$ $0.8 \mu \mathrm{m}$ wide $(\bar{x}=0.55 \mu \mathrm{m}, \mathrm{n}=30)$, smooth. Conidiogenous cells integrated, $12-17 \times 3-5$ $\mu \mathrm{m}(\bar{x}=14.7 \times 0.67 \mu \mathrm{m}, \mathrm{n}=10)$, pale olivaceous or brown; conidiogenous loci inconspicuous. Conidia solitary, obclavate, straight to moderately curved, $44-80 \times 3-5 \mu \mathrm{m}$ $(\bar{x}=64 \times 4.1 \mu \mathrm{m}, \mathrm{n}=30), 3-8$-septate, slightly constricted at the septa, pale olivaceous, wall $0.5-0.8 \mu \mathrm{m}$ wide $(\bar{x}=0.53$ $\mu \mathrm{m}, \mathrm{n}=30$ ), smooth, obtuse at the apex, with long obconically truncate base, hilum unthickened, not darkened.

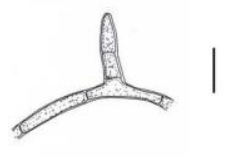

1
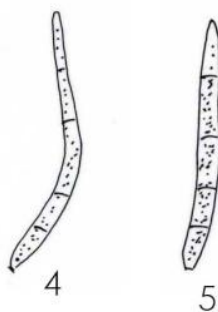
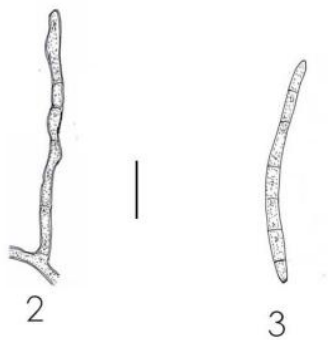

3

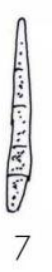

Fig. 98 - Pseudocercospora tremicola on Trema orientalis: 1-2. External hyphae with attached conidiophores. 3-7. Conidia. Bar: 1$7=10 \mu \mathrm{m}$.

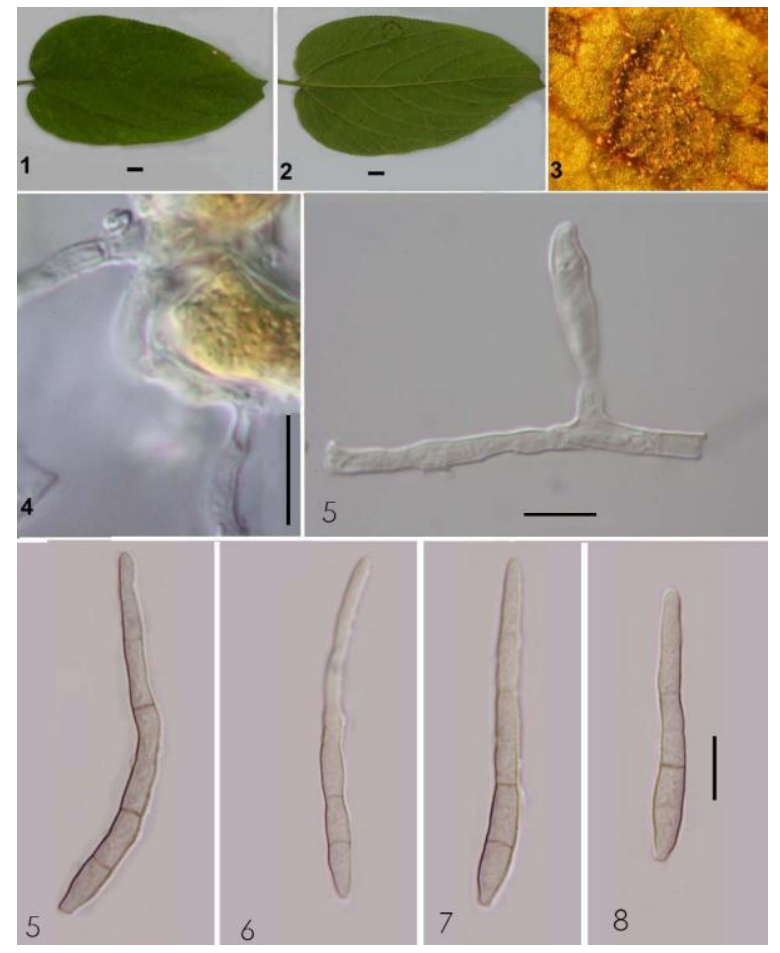

Fig. 99 - Pseudocercospora tremicola on Trema orientalis from leaf spots: 1-2. Leaf spots on host leaves (1. upper surface, 2. lower surface). 3. Colonies. 4. Internal hyphae with attached conidiophore. 5-8. Conidia. Bars: 1$2=10 \mathrm{~mm}$. 3. Not to scale. $4-8=10 \mu \mathrm{m}$.

Known hosts - Trema cannabina Lour., T. guineensis (Schum. \& Thonn.) Ficalho, $T$. orientalis (L.) Blume, T. politoria (Planch.) Blume (Cannabaceae).

Known distribution - Africa: Gabon, Ghana, Ivory Coast, Sierra Leone; Asia: India, Laos, Singapore, Taiwan, Thailand.

Material examined - Chiang Rai Province, Tasud Muang District, Sri Pangsang Village, on leaves of Trema orientalis (Cannabaceae), 25 January 2010, P. Phengsintham (MFLU10 0288).

Notes - In the Thailand collection the conidiophores are 30-110 $\times 3-4 \mu \mathrm{m}$ and the conidia are 44-80 $\times 4-5 \mu \mathrm{m}$, which is similar to those reported in Ellis (1971) and Hsieh \& Goh (1990) [conidiophores 25-55 × 3.5-5.5 $\mu \mathrm{m}$, conidia 20-90 $\times$ 4-5 $\mu \mathrm{m}]$. Pseudocercospora tremae-orientalis (S.H. Sun) Deighton is distinguished from $P$. tremicola by forming stromata, 30-35 $\mu \mathrm{m}$ diam., and fasciculate conidiophores. Superficial mycelium with solitary conidiophores is lacking (Hsieh \& Goh 1990). 
Literature - Deighton (1979: 9); Hsieh \& Goh (1990: 333); Guo \& Hsieh (1995: 334); Guo et al. (1998: 400); Crous \& Braun (2003: 408).

Zasmidium cassiicola (S. Mishra, A.K. Srivast. \& Kamal) Kamal, Cercosporoid Fungi of India: 239, 2010.

Figs 100-101.

= Stenella cassiicola S. Mishra, A.K. Srivast. \& Kamal, Mycol. Res. 103: 268, 1999.

Leaf spots variable, more or less irregularly orbicular, 1-8 mm diam., typically deep brown. Colonies hypophyllous, conspicuous. Mycelium external; hyphae branched, $2-4 \mu \mathrm{m}$ wide $(\bar{x}=3.2 \mu \mathrm{m}, \mathrm{n}=9)$, septate, constricted at the septa, distance between septa 8-30 $\mu \mathrm{m}(\bar{x}=18.56 \mu \mathrm{m}, \mathrm{n}=9)$, pale olivaceous-brown, thin-walled $0.3-0.5 \mu \mathrm{m}$ wide ( $\bar{x}=0.41 \mu \mathrm{m}, \mathrm{n}=9$ ), verruculose. Stromata absent. Conidiophores borne on external mycelial hyphae, unbranched, cylindrical, $30-117 \times 3-4 \mu \mathrm{m}(\bar{x}=65.9 \times$ $3.17 \mu \mathrm{m}, \mathrm{n}=18)$, 3-8-septate, distance between septa $7-25 \mu \mathrm{m}(\bar{x}=15.8 \mu \mathrm{m}, \mathrm{n}=30)$, mid pale golden brown, wall $0.5-0.8 \mu \mathrm{m}(\bar{x}=$ $0.48 \mu \mathrm{m}, \mathrm{n}=30$ ), smooth. Conidiogenous cells integrated, terminal or intercalary, 7-20 $\times 2-4$ $\mu \mathrm{m}(\bar{x}=13 \times 2.63 \mu \mathrm{m}, \mathrm{n}=8)$, cylindrical, swollen and curved at the apex; conidiogenous loci formed as minute, dark or refractive scars on lateral and terminal denticles, $1-2 \mu \mathrm{m}$ diam. $(\bar{x}=1.4 \mu \mathrm{m}, \mathrm{n}=7)$, giving rise to branched conidial chains, wall $0.3-0.5 \mu \mathrm{m}$ wide $(\bar{x}=0.4$ $\mu \mathrm{m}, \mathrm{n}=7$ ), thickened, darkened. Conidia solitary or catenate, sometimes ellipsoidalovoid or subcylindrical, but mostly slightly obclavate, straight or slightly curved or sinuous, $11-70 \times 2-4 \mu \mathrm{m}(\bar{x}=34.16 \times 2.9$ $\mu \mathrm{m}, \mathrm{n}=24), 1-5$-septate, pale olivaceous, wall $0.3-0.5 \mu \mathrm{m}$ wide $(\bar{x}=0.33 \mu \mathrm{m}, \mathrm{n}=24)$, smooth or finely verruculose; apex rounded or subtruncate, $1-1.5 \mu \mathrm{m}$ wide, wall $0.3-0.5 \mu \mathrm{m}$ wide; base short tapered at the base to the hilum, $1-2 \mu \mathrm{m}$ wide $(\bar{x}=1.3 \mu \mathrm{m}, \mathrm{n}=9$ ), wall $0.3-0.5 \mu \mathrm{m}$ wide $(\bar{x}=0.41 \mu \mathrm{m}, \mathrm{n}=9)$, thickened and darkened.

Colonies on PDA after 3 weeks at $25^{\circ} \mathrm{C}$ with spreading mycelium, surface ridged, black and wavy in the centre and grey margin, reaching 5-15 $\mathrm{mm}$ diam.; hyphae often constricted at the septa, distance between septa

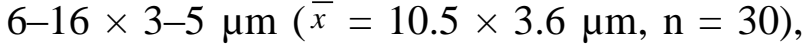
thin-walled, $0.3-0.5 \mu \mathrm{m}$ wide $(\bar{x}=0.45 \mu \mathrm{m}, \mathrm{n}$ $=30$ ), hyaline, smooth or verruculose; conidia not formed in culture.

Known host - Cassia fistula L. (Fabaceae).

Known distribution - Asia: India, Thailand.

Material examined - Chiang Rai Province, Muang District, Sri Pangsang Village, on leaves of Cassia fistula (Fabaceae), 16 January 2010, P. Phengsintham (MFLU100324).

Notes - The collection from Thailand agrees well with the Indian Zasmidium cassiicola (Braun et al., 2006; Mishra et al., 1999). This is the first record outside India.

Zasmidium cassiae-fistulae (Mishra et al., (1999) is a similar species but differs in forming its conidia consistently singly (Braun et al., 2006).

Literature - Mishra et al. (1999); Braun et al. (2006).

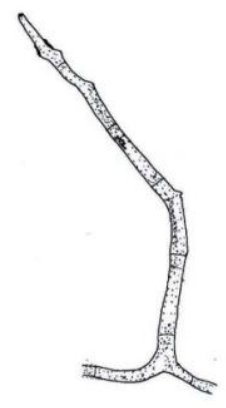

1

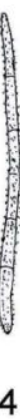

4

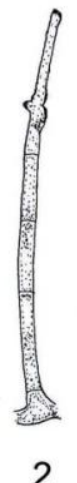

2

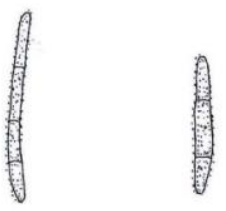

6

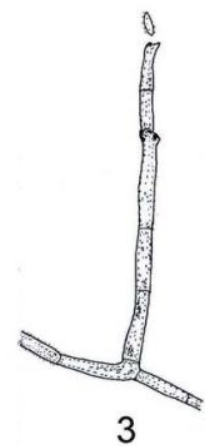

3

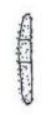

7
Fig. 100 - Zasmidium cassiicola on Cassia fistula: 1-3. External mycelia with attached conidiophore. 4-7. Conidia. Bars $=10 \mu \mathrm{m}$. 


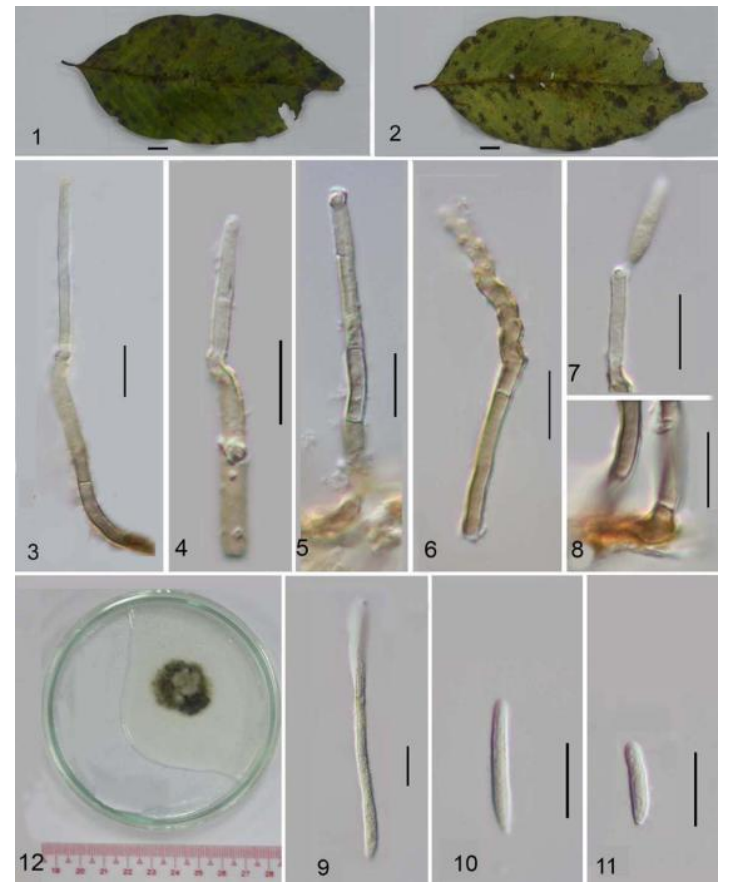

Fig. 101 - Zasmidium cassiicola on Cassia fistula from leaf spot: 1-2 Leaf spots on host leaves (1. upper surface, 2. lower surface). 3-6. Conidiophores. 7. Apex with attached conidium. 8. External mycelium. 9-11. Conidia. 12. Living culture. Bars: $1-2=10$ $\mathrm{mm}, 3-11=10 \mu \mathrm{m}$.

\section{Additional list of cercosporoid fungi}

Cercospora alocasiae Goh \& W.H. Hsieh, Trans. Mycol. Soc. Republ. China 2: 86-87, 1987.

Material examined - Chiang Rai Province, Maechan District, Doitung National Park, Fallow forest, on leaves of Alocasia macrorrhiza (L.) G. Don (Araceae), 8 August 2009, P. Phengsintham (P427).

Cercospora begoniae Nori, Lecture on plant diseases (Shokubutsu Bybai Kowa), 2: 181, 1916.

Material examined - Chiang Rai Province, Houay Kangpa National Park, on leaves of Begonia inflata C.B. Clarke (Begoniaceae), 20 November 2009, P. Phengsintham (P468).

Cercospora bidentis Tharp. Mycologia 9: 108, 1917.

Material examined - Chiang Rai
Province, Meuang District, Sri Pangxang Village, Fallow forest, on leaves of Bidens pilosa L. (Asteraceae), 18 August 2009, P. Phengsintham (P436).

Cercospora brassicicola Henn., Bot. Jahrb. Syst. 37: 166, 1905.

Material examined - Chiang Rai Province, Doi Tung National Park, on leaves of Brassica integrifolia (H. West) Rupr. (Brassicaceae), 4 December 2009, P. Phengsintham (P482).

Cercospora capsicigena Bhartiya, Dubey \& S.K. Singh, Indian Phytopathol. 5(3): 149, 2000.

Material examined - Chiang Mai Province, Maeteng District, Phadeng Village, on leaves of Capsicum annuum L. (Solanaceae), 17 July 2007, P. Phengsintham (P289).

Cercospora cocciniae Munjal, Lall \& Chona, Indian Phytopathol. 12: 86, 1959.

Material examined - Chiang Rai Province, Sri Pangsang Village, on leaves of Coccinia indica Wight \& Arn. (Cucurbitaceae), 4 August 2009, P. Phengsintham (P408).

Cercospora duranticola Phengsintham, Chukeatirote, McKenzie, K.D. Hyde \& U. Braun, Current Research in Environmental \& Applied Mycology 3(1): 59, 2013.

Material examined - Chiang Rai Province, Meuang District, Sri Pangsang Village, Urban area, on leaves of Duranta repens L. (Verbenaceae), 17 August 2009, P. Phengsintham (P435).

Cercospora erechtitis G.F. Atk., J. Elisha Mitch. Sci. Soc. 8: 66, 1892.

Material examined - Chiang Rai Province, Wiangpapao, on leaves of Erechtites valerianifolius (Link ex Spreng.) DC. (Asteraceae), 28 May 2008, P. Phengsintham (P312).

Cercospora ipomoeae G. Winter, Hedwingia 26: 34, 1887.

Material examined - Chiang Rai Province, Muang District, Sri Pangsang Village, Rice paddy, on leaves of Ipomoea 
aquatica Forssk. (Convolvulaceae), 29 August 2010, P. Phengsintham (P627).

\section{Cercospora sp.}

Material examined - Chiang Rai Province, Doi Tung National Park, Fallow forest, on leaves of Oroxylum indicum (L.) Kurz (Bignoniaceae), 22 August 2009, P. Phengsintham (P438).

Cercospora physalidis Ellis, Amer. Naturalist 16: 810, 1882.

Material examined - Chiang Rai Province, Tadsack Waterfall, on leaves of Physalis angulata L. (Solanaceae), 3 May 2006, P. Phengsintham (P443).

Cercospora zinniae Ellis \& G. Martin, J. Mycol. 1: 20, 1885.

Material examined - Chiang Rai Province, Meuang District, Sri Pangsang Village, on leaves of Zinnia elegans Jacq. (Asteraceae), 15 September 2009, P. Phengsintham (P453).

Passalora aenea (Cif.) U. Braun \& Crous, in Crous and Braun, Mycosphaerella and its anamorphs: 1. Names published in Cercospora and Passalora. CBS Biodiversity Series 1: 46, 2003.

Material examined - Chiang Rai Province, Muang District, Sri Pangsang village, on leaves of Senna siamea (Lam.) H.S. Irwin \& Barneby (Fabaceae), 9 January 2010, P. Phengsintham (P518).

Passalora bougainvilleae (Munt.-Cvetk.) R.F. Castañeda \& U. Braun, Cryptog. Bot. 2: 291, 1991.

Material examined - Chiang Rai Province, Doi Tung National Park, Village area, on leaves of Bougainvillea spectabilis Willd. (Nyctaginaceae), 8 August 2009, P. Phengsintham (P342).

Passalora henningsii (Allesch.) R.F. Castañeda \& U. Braun, Cryptog. Bot. 1(1): 46, 1989.

Material examined - Chiang Rai Province, Doi Tung National Park, Village area, on leaves of Manihot utilissima Pohl (Euphorbiaceae), September 2009, P.
Phengsintham (P445).

Passalora perfoliati (Ellis \& Everh) U. Braun $\&$ Crous, in Crous and Braun, Mycosphaerella and its anamorphs: 1. Names published in Cercospora and Passalora. CBS Biodiversity Series 1: 46, 2003.

Material examined - Chiang Mai, Maeteng District, Phadeng Village, Fallow forest, on leaves of Chromolaena sp. (Asteraceae), 17 July 2007, P. Phengsintham (P114); Chiang Rai, Muang District, Sri Pangsang Village, Fallow forest, on leaves of Chromolaena sp. (Asteraceae), 28 May 2008, P. Phengsintham (P214).

Pseudocercospora catappae (Henn.) X.J. Liu \& Y.L. Guo, Mycosystema 2: 230, 1989.

Material examined - Chiang Rai Province, Muang District, Sri Pangsang Village, on leaves of Terminalia tomentosa Wight \& Arn. (Combretaceae), 20 November 2010, P. Phengsintham (P653).

Pseudocercospora centrosematicola (J.M. Yen \& G. Lim) J.M. Yen, Gard. Bull., Singapore 33: $171,1980$.

Material examined - Chiang Rai Province, Muang District, Sri Pangsang Village, on leaves of Centrosema pubescens Benth. (Fabaceae), 20 November 2010, P. Phengsintham (P654).

Pseudocercospora cotizensis (A.S. Mull. \& Chupp) Deighton, Mycol. Pap. 140: 142, 1976. Material examined - Chiang Rai Province, Muang District, Sri Pangsang Village, Village area, on leaves of $C$. uncinella subsp. elliptica (Roxb.) Polhill. (Fabaceae), 31 August 2010, P. Phengsintham (P629).

Pseudocercospora duabangae M.D. Mehrotra \& R.K. Verma. Mycol. Res. 95 (10): 1163 1168, 1991.

Material examined - Chiang Rai Province, Khounkon water fall, on leaves of Duabanga grandiflora (Roxb. ex DC.) Walp. (Lythraceae), 18 December 2009, P. Phengsintham (MFLU10 0287).

Pseudocercospora eupatorii-formosani U. Braun \& Bagyan., Sydowia 51: 8, 1999. 
Material examined - Chiang Rai Province, Sri Pangsang Village, Village area, on leaves of Chromolaena odorata (L.) R.M. King \& H. Rob. (Asteraceae), 16 January 2010, P. Phengsintham (P527).

Pseudocercospora fuligena (Roldan) Deighton, Mycol. Pap. 140: 144, 1976.

Material examined - Chiang Rai Province, Muang District, Sri Pangsang Village, Garden, on leaves of Lycopersicon esculentum Mill. (Solanaceae), 3 June 2010, P. Phengsintham (P578).

Pseudocercospora getoniae Phengsintham, Chukeatirote, McKenzie, K.D. Hyde \& U. Braun, Current Research in Environmental \& Applied Mycology 3(1): 105, 2013.

Material examined - Chiang Rai Province, Tadsack Water Fall, on leaves of Getonia floribunda Roxb. (三 Calycopteris floribunda (Roxb.) Lam. ex Poir.) (Combretaceae), 27 August 2009, P. Phengsintham (444).

Pseudocercospora jussiaeae (G.F. Atk.) Deighton, Mycol. Pap. 140: 146, 1976.

Material examined - Chiang Rai Province, Muang District, Sri Pangsang Village, on leaves of Ludwigia prostrata Roxb. (Onagraceae), 20 November 2011, P. Phengsintham (P656).

Pseudocercospora lythracearum (Heald \& F.A. Wolf) X.J. Liu \& Y.L. Guo, Acta Mycol. Sin. 11: 294, 1992.

Material examined - Chiang Rai Province, Muang District, Ban Sri Pangsang Village, on leaves of Lagerstroemia macrocarpa Wall. (Lythraceae), 4 August 2010, P. Phengsintham (P611).

Pseudocercospora musae (Zimm.) Deighton, Mycol. Pap. 140: 148, 1976.

Material examined - Chiang Rai Province, Muang District, Sri Pangxang Village, Musa paradisiaca L. (Musaceae), 15 January 2010, P. Phengsintham (P520).

Pseudocercospora paraguayensis (Tak. Kobay.) Crous, Mycotaxon 57: 270, 1996.
Material examined - Chiang Rai Province, Muang District, Sri Pangsang Village, on leaves of Eucalyptus sp. (Myrtaceae), 16 January 2010, P. Phengsintham (P530).

Pseudocercospora puerariicola (W. Yamam.) Deighton, Mycol. Pap. 140: 151, 1976.

Material examined - Chiang Rai Province, Muang District, Sri Pangsang Village, on leaves of Pueraria phaseoloides (Roxb.) Benth. (Fabaceae), 20 November 2011, P. Phengsintham (P567).

Pseudocercospora sphaerellae-eugeniae (Sacc.) Crous, Alfenas \& R.W. Barreto, Mycotaxon 64: 425, 1997.

Material examined - Chiang Rai Province, Muang District, Sri Pangsang Village, on leaves of Syzygium cuminii (L.) Skeels (Myrtaceae), 20 November 2011, P. Phengsintham (P658).

Pseudocercospora stahlii (F. Stevens) Deighton, Mycol. Pap. 140: 82, 1976.

Material examined - Chiang Rai Province, Muang District, Sri Pasang Village, on leaves of Passiflora foetida L. (Passifloraceae), 11 September 2009, P. Phengsintham (P452).

Pseudocercospora stizolobii (Syd. \& P. Syd.) Deighton, Mycol. Pap. 140: 153, 1976. Material examined - Chiang Rai Province, Muang District, Sri Pangsang Village, on leaves of Mucuna pruriens (L.) DC. (Fabaceae), 20 November 2011, P. Phengsintham (P655).

Pseudocercospora trichophila (F. Stevens) Deighton var. punctata U. Braun \& Urtiaga, Mycosphere 3(3): 322, 2012.

Material examined - Chiang Rai Province, Muang District, Sri Pangsang Village, on leaves of Solanum sp. (Solanaceae), 22 August 2009, P. Phengsintham (440).

Pseudocercospora wrightiae (Thirum. \& Chupp) Deighton, Mycol. Pap. 140: 156, 1976. Material examined - Chiang Rai Province, Muang District, Sri Pangsang Village, on leaves of Wrightia pubescens 
Blume (Apocynaceae), 23 December 2009, P. Phengsintham (P496).

Zasmidium suregadae Phengsintham, K.D. Hyde \& U. Braun, Cryptog. Mycol. 30(3): 258, 2009.

Material examined - Chiang Mai Province, Maeteng District, Phadeng Village, Fallow forest, on leaves of Suregada multiflora (A. Juss.) Baill. (Euphorbiaceae), 12 August 2011, P. Phengsintham (P640).

Cladosporium colocasiae Sawada, Trans. Nat. Hist. Soc. Taiwan25: 125, 1916.

Material examined - Chiang Mai Province, Metaeng District, on leaves of Colocasia esculenta (L.) Schott (Araceae), 17 July 2007, P. Phengsintham (P285); Chiang Rai Province, Mechanh District, Doi Tung National Park, on leaves of Colocasia esculenta (L.) Schott (Araceae), 4 December 2009, P. Phengsintham (P483).

\section{Acknowledgements}

The authors would like to thank the Mushroom Research Foundation (MRF) for financial support. Special thanks go to the MRF organizers and members of Prof. K.D. Hyde's laboratory, Mae Fah Luang University (MFU) for their assistance. The authors would like also thank to Prof. Dr. Cai Lei, State Key Laboratory of Mycology, Institute of Microbiology, Chinese Academy of Sciences, Beijing 100101, P.R. China for support for molecular phylogeny studies.

\section{References}

Boedijn KB. 1961 - The genus Cercospora in Indonesia. Nova Hedwigia 3, 411-436.

Braun U. 2001 - Revision of Cercospora species described by K.B. Boedijn. Nova Hedwigia 73(3-4), 419-436.

Braun U. 2003 - Miscellaneous notes on some cercosporoid hyphomycetes. Bibliotheca Lichenologica 86, 79-98.

Braun U, Crous PW, Kamal 2006 - New species of Pseudocercospora, Pseudocercosporella, Ramularia and Stenella (cercosporoid hyphomycetes). Mycological Progress 2, 197-208.
Choi YW, Hyde KD, Ho WH. 1999 - Single spore isolation of fungi. Fungal Diversity 3, 29-38.

Chupp C. 1954 - A Monongraph of the Fungus Genus Cercospora. Ithaca, New York. Published by the author.

Crous PW, Braun U. 2003 - Mycosphaerella and its anamorphs: Names published in Cercospora and Passalora. CBS Biodiversity Series 1. CBS Utrecht, The Netherlands.

Deighton FC. 1967 - Studies on Cercospora and allied genera. II. Passalora, Cercosporidium, and some species of Fusicladium on Euphorbia. Mycological Papers 112, 1-80.

Deighton FC. 1971 - Studies on Cercospora and allied genera. III. Centrospora. Mycological Papers 124, 1-13.

Deighton FC. 1973 - Studies on Cercospora and allied genera. IV. Cercosporella Sacc., Pseudocercosporella gen. nov. and Pseudocercosporidium gen. nov. Mycological Papers 133, 1-62.

Deighton FC. 1976 - Studies on Cercospora and allied genera. VI. Pseudocercospora Speg., Pantospora Cif., and Cercoseptoria Petr. Mycological Papers 140, 1-168.

Deighton FC. 1979 - Studies on Cercospora and allied genera. VII. New species and redispositions. Mycological Papers 144, $1-56$.

Deighton FC. 1987 - New species of Pseudocercospora and Mycovellosiella, and new combination into Pseudocercospora and Phaeoramularia. Transactions of the British Mycological Society $88,365-391$.

Ellis MB. 1971 - Dematiaceous hyphomycetes. Commonwealth Mycological Institute Kew, Surrey, England.

Ellis MB. 1976 - More dematiaceous hyphomycetes. Commonwealth Mycological Institute Kew, Surrey, England.

Giatgong P. 1980 - Host index of plant disease of Thailand. Mycology Section. Plant Pathology and Microbiology Division. Department of Agriculture, Bangkok, Thailand.

Groenewald JZ, Nakashima C, Nishikawa J, Shin HD, Park JH, Jama AN, 
Groenewald M, Braun U, Crous PW. 2012 - Species concepts in Cercospora: spotting the weeds among the roses. Studies in Mycology 75, 115-170.

Guo YL, Liu XJ, Hsieh WH. 1998 Pseudocercospora. Flora Fungorum Sinicorum. Vol. 9. Beijing, P.R. China.

Guo YL, Hsieh WH. 1995 - The genus Pseudocercospora in China. Mycosystema Monographicum Series 2, 1388.

Hsieh WH, Goh TK. 1990 - Cercospora and Similar Fungi from Taiwan. Maw Chang Book Company, Taipei, Taiwan.

Hywel-Jones NL. $2001-$ A review of invertebrate pathogenic Clacipitaceae of Thailand. BRT 2001 Research Report, Bangkok, 34-41.

Hywel-Jones NL, Boonpratuang T. 2001 - An updated preliminary checklist of fungi recorded from Thailand. BIOTEC database.

Jones EBG, Hyde KD. 2004 - Introduction to Thai fungal diversity. In Thai Fungal Diversity. BIOTEC, Thailand, 7-35.

Kirschner R, Chen CJ. 2007 - Foliicolous hyphomycetes from Taiwan. Fungal Diversity 26, 219-237.

Meeboon J. 2009 - Diversity and phylogeny of true cercosporoid fungi from northern Thailand. Ph.D. Thesis, Chiang Mai University, Thailand (Unpublished).

Meeboon J, Hidayat I, To-anun C. 2007 Annotated list of cercosporoid fungi in northern Thailand. Journal of Agricultural Technology 3, 51-63.

Meeboon J, Hidayat I, To-anun C, Nakashima C. 2008 - Cercosporoid fungi from Thailand II. New species of Cercospora and Passalora. Sydowia 60(2), 253260.

Mishra S, Srivastava KK, Kamal 1999 Further additions to Stenella from India and Nepal. Mycological Research 103, 268-270.

Nakashima C, Motohashi K, Meeboon J, Toanun C. 2007 - Studies on Cercospora and allied genera in northern Thailand. Fungal Diversity 26, 257-270.

Petcharat V, Kajanamaneesathian M. 1989 Species of plant pathogenic Cercospora in Southern Thailand. Thai Phytopathology 9, 23-27.

Pons N, Sutton BC. 1988 - Cercospora and similar fungi on yams (Dioscorea species). Mycological Papers 160, 178.

Prihastuti H, Cai L, Chen H, McKenzie EHC, Hyde KD. 2009 - Characterization of Colletotrichum species associated with coffee berries in Northern Thailand. Fungal Diversity 39, 89-109.

Raška I, Koberna K, Malínský J, Fidlerová H, Mašata M. 2004 - The nucleolus and transcription of ribosomal genes. Biology of the Cell 96, 579-594.

Rostrup E. 1902 - Fungi. In flora of Koh Chang. Botanik Tidsskrift 24, 355-367.

Saccardo PA. 1902 - Sylloge Fungorum omnium hucusque cognitorum, Vol. 16. Padova.

Saccardo PA. 1913 - Sylloge Fungorum omnium hucusque cognitorum, Vol. 22. Padova.

Saccardo PA. 1931 - Sylloge Fungorum omnium hucusque cognitorum, Vol. 25. Avellino.

Saccardo PA. 1972 - Sylloge Fungorum omnium hucusque cognitorum, Vol. 26 (Trotter, A. ed., published by Cash, K.), Johson Reprint Corporation, New York, London.

Shin HD, Kim JD. 2001 - Cercospora and allied genera from Korea. Plant Pathogens from Korea 7, 1-302.

Shirouzu T, Hirose D, Fukasawa Y, Tokumasu S. 2009 - Fungal succession associated with the decay of leaves of an evergreen oak, Quercus myrsinaefolia. Fungal Diversity 34, 87-109.

Sontirat P, Pitakpraiwan P, Choonbamroong W, Kueprakone U. 1980 - Plant pathogenic cercosporoid in Thailand. Department of Agriculture, Ministry of Agriculture and Cooperative. Bangkok, Thailand.

Tanticharoen M. 2004 - Introduction to Thai biodiversity. In Thai Fungal Diversity. BIOTEC, Thailand, 1-6.

Than PP, Jeewon R, Hyde KD, Pongsupasamit S, Mongkolporn O, Taylor PWJ. 2008 Characterization and pathogenicity of Colletotrichum species associated with 
anthracnose on chilli (Capsicum spp.) in Thailand, Plant Pathology 57, 562572.

To-anun C, Hidayat I, Meeboon J. 2011 Genus Cercospora in Thailand: Taxonomy and phylogeny (with a dichotomous key to species). Plant Pathology \& Quarantine 1(1), 11-87.

To-anun C, Nguenhom J, Meeboon J, Hydayat I. 2009 - Two fungi associated with necrotic leaflets of areca palm (Areca catechu). Mycological Progress 8, 115121.

Vasudeva RS. 1963 - India Cercosporae. Indian Council of Agricultural Research. New Delhi.
Wannathes N, Desjardin DE, Hyde KD, Perry BA, Lumyong S. 2009 - A monograph of Marasmius (Basidiomycota) from northern Thailand based on morphological and molecular (ITS sequences). Fungal Diversity 37, 209-306.

Wu CY, Robens PH. 1988 - Flora of China, Vol. 18, Beijing.

Xu L, Guo LT. 2003 - Studies on Cercospora and allied genera in China XIII. Mycosystema 22, 6-8.

Yen JM, Lim G. 1980 - Cercospora and allied genera of Singapore and the Malay Peninsula. Garden Bulletin Singapore 33, 175-176. 